\title{
Elucidating a Common Mechanism of Proteasome Impairment in Neurodegenerative Disease and its Pharmacological Intervention
}

\author{
Tiffany Ann Thibaudeau \\ WVU, tthibaud@mix.wvu.edu
}

Follow this and additional works at: https://researchrepository.wvu.edu/etd

Part of the Biochemistry Commons

\section{Recommended Citation}

Thibaudeau, Tiffany Ann, "Elucidating a Common Mechanism of Proteasome Impairment in Neurodegenerative Disease and its Pharmacological Intervention" (2019). Graduate Theses, Dissertations, and Problem Reports. 3780.

https://researchrepository.wvu.edu/etd/3780

This Dissertation is protected by copyright and/or related rights. It has been brought to you by the The Research Repository @ WVU with permission from the rights-holder(s). You are free to use this Dissertation in any way that is permitted by the copyright and related rights legislation that applies to your use. For other uses you must obtain permission from the rights-holder(s) directly, unless additional rights are indicated by a Creative Commons license in the record and/ or on the work itself. This Dissertation has been accepted for inclusion in WVU Graduate Theses, Dissertations, and Problem Reports collection by an authorized administrator of The Research Repository @ WVU.

For more information, please contact researchrepository@mail.wvu.edu. 


\title{
Elucidating a Common Mechanism of Proteasome Impairment in Neurodegenerative Disease and its Pharmacological Intervention
}

\section{Tiffany Ann Thibaudeau}
Dissertation submitted to the School of Medicine at West Virginia University in partial fulfillment of the requirements for the degree of

Doctor of Philosophy in Biochemistry and Molecular Biology

\author{
David M. Smith, Ph.D., Chair and Mentor \\ Michael Schaller, Ph.D. \\ Michael Gunther, Ph.D. \\ Andrew Shiemke, Ph.D. \\ Justin Legleiter, Ph.D.
}

Biochemistry and Molecular Biology Program

Morgantown, West Virginia

2019

Keywords: proteasome, protein degradation, proteasome gate, neurodegeneration, protein oligomers

Copyright 2019 Tiffany A. Thibaudeau 


\section{Abstract \\ Elucidating a Common Mechanism of Proteasome Impairment in Neurodegenerative Disease and its Pharmacological Intervention}

Tiffany Ann Thibaudeau

Proteostasis is maintained by several systems in the cell including the ubiquitin-proteasome system (UPS), chaperones, chaperone-mediated autophagy, and macroautophagy. The UPS is the principle route for the degradation of intracellular misfolded, damaged, or unneeded cellular proteins and has a critical role essential every cell process, including: cell cycle progression, transcriptional regulation, genome integrity, apoptosis, immune responses, and neuronal plasticity. When the efficiency of protein degradation is perturbed, misfolded and damaged protein aggregates can accumulate to toxic levels and cause neuronal dysfunction, which may underlie many neurodegenerative diseases. It is widely appreciated that soluble oligomers of misfolded proteins (e.g. A $\beta$, Alzheimer's; $\alpha$-Syn, Parkinson's; huntingtin with polyglutamine expansion, Huntington's) and loss of proteostasis are the key drivers of disease development and progression. We show that three different proteins from Alzheimer's, Parkinson's, and Huntington's disease that misfold and oligomerize into a shared three-dimensional structure potently impair the proteasome. Detailed mechanistic analysis demonstrates that these oligomers inhibit the $20 \mathrm{~S}$ proteasome through allosteric impairment of the substrate-gate. To investigate the feasibility of proteasome gate-activation as a therapeutic strategy, we characterized the mechanism of proteasome gate activation by the conserved $\mathrm{HbYX}$-motif found in proteasome activator complexes at the molecular level. Based on these observations, we developed a novel proteasome gate-activating molecule as a research tool to probe proteasome function in vitro and in vivo. Together, our results provide a novel molecular model for oligomer-driven proteasome impairment in neurodegenerative disease and demonstrate the feasibility of designing drug-like molecules that activate proteasome function patients with neurodegenerative disease. 


\section{Dedication}

I dedicate this to my fiancé Steve, my mother Jeanie, and my sister Christina. Your love, encouragement, support, and confidence in my ability to succeed was a constant light even on the darkest days. 


\section{Acknowledgements}

My journey toward a Ph.D. in Biochemistry took a long, winding, and unusual road. I studied the principles of kitchen and bath design, construction, mechanical, plumbing and electrical systems. I rebuilt transmissions and repaired engines. I studied business administration and finance, before ultimately deciding on a Bachelor of Arts in psychology degree program. To MIT OpenCourseWare: the audio lectures by Dr. Gerald Schneider and Dr. Eric Lander ultimately changed the trajectory of my education and future career (and gave me something to do during the hour-long commutes to work!). Thank you to Dr. Gerald Schneider to introducing me to the amazing world of neuroscience. I had zero interest in the basic sciences until I "met" Dr. Lander, who's enthusiasm for molecular biology was infectious. My mind was blown by the cleverness of early molecular genetic methods and I was hooked on science.

I would like to thank my fiancé Steve for supporting me through my craziness, feeding me delicious food so I didn't have to survive on bar-be-que chicken alone, and loving me unconditionally. I never would have imagined that l'd meet my love and my future in Morgantown, but here we are. You've shown me what it means to be part of a team and I love you more every day.

To my mother, Jeanie: thank you for your hard work and countless sacrifices when Christina and I were children. You showed me how to work towards my goals and persevere, no matter what challenges stood in the way. Growing up with your unconditional love and unwavering emotional support gave me the guts to not only pursue my dreams but achieve them as well (even the seemingly impossible ones!). To my sister and best friend, Christina: no matter what, you always find a way to put me in a better mood (usually through your awesome sense of humor) and that's priceless. To my nephew, Alexander: The picture of 1-year old you, smiling in your Cummins diesel shirt, stayed on my lab bench for years and looking at you would always brighten my mood. To Forrest and Ezzie, the newest additions to my family: Videos of your silly and adorable antics were always a bright spot in my day.

To my mentor, Dr. David M. Smith: thank you for believing in me and providing support even when others did not. I am grateful for your willingness to mentor a new student without any formal science education or training and thank you for showing me how to use a pipette! Thank you for giving me the freedom to pursue my academic interests and for gently guiding me when l've strayed too far. The skills l've learned under your guidance have set me up for a successful career. To Jane, our amazing lab manager: thank you for being a constant source of knowledge and friendship, and for being an irreplaceable ally.

To my lab mates Ray and Taylor, , thank you for being there to share in my frustrations and elations and letting me rant endlessly about the thing that's bothering me that day.

To my work-wife Jess, thank you for convincing me to join the biochemistry department, and thank you for your friendship and support (especially during the early years when the going was tough), it was invaluable and helped me make it thought those tough times.

Thank you to all my committee members, you have been incredibly helpful and constructive in shaping my understanding of the field. Your advice has been invaluable to reaching this point. Thank you to Dr. Michael Schaller, I appreciate your support and encouragement during my early years at WVU. Thank you to the members of the Old Guys' Journal Club, I learned so much from all of you and no doubt will be a better scientist because of it.

Lastly, I would like to thank the Biochemistry and Molecular Biology program and West Virginia University for the opportunity to earn my Ph.D. You have shaped me into a capable scientist that I hope you will be proud to count among your alumni. 


\section{Table of Contents}

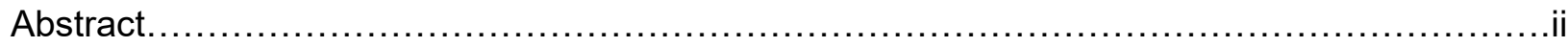

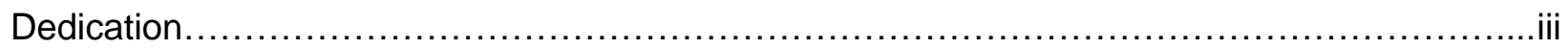

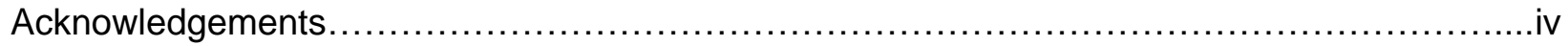

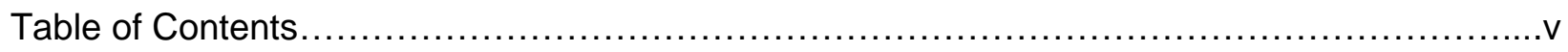

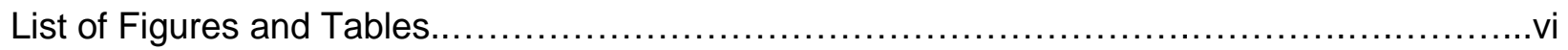

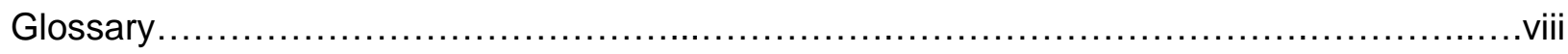

Literature Review - Part 1 - Neurodegenerative disease and the proteasome...................1

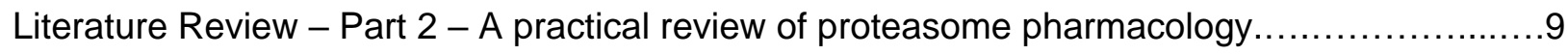

Study 1: A common mechanism of proteasome impairment by neurodegenerative disease-

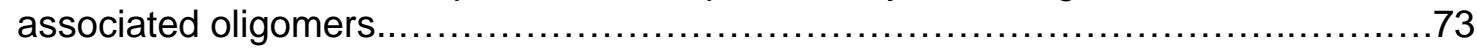

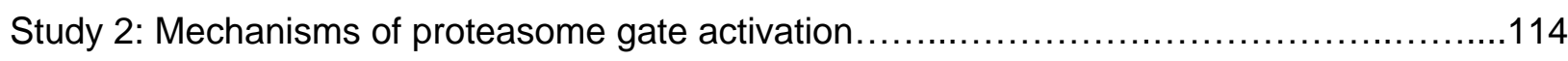

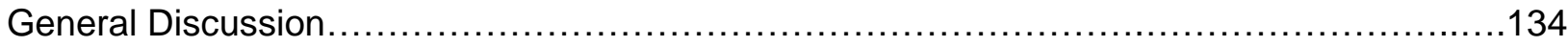

Appendix

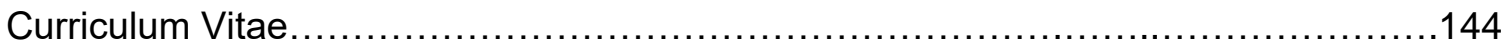




\section{List of Figures and Tables}

\section{Literature Review - Neurodegenerative Disease}

Figures

1. Characteristic neurodegenerative disease neuropathological lesions

2. Flowchart for pathway of protein aggregation

3. The proteostasis network

4. Protein misfolding and proteasome impairment

\section{Literature Review - Proteasome Pharmacology}

Figures

1. The ubiquitin proteasome pathway

2. Proteasome structure and function

3. Examples of cellular pathways that depend on proteasome function.

4. Proteasome inhibitors and the $\beta 5$ binding site

\section{Tables}

1. Experimental pitfalls

2. Fluorogenic peptide substrates

3. Cellular proteasome substrates

4. Proteasome inhibitors in clinical testing

\section{Study 1}

Figures

1. A specific conformation of soluble oligomers potently inhibits the mammalian $20 \mathrm{~S}$ proteasome

2. Generation of stabilized $A 11+$ oligomers $\left(A \beta^{\star} 56\right)$ for mechanistic evaluation of proteasome inhibition

3. The $A 11(+)$ oligomers bind to the $20 \mathrm{~S}$ proteasome and impair opening of the substrate gate

4. $\mathrm{A} 11(+)$ oligomers cannot inhibit PA26 or PA28 $\alpha \beta$ induced gate opening

5. $A \beta^{\star} 56$ oligomers inhibit ubiquitin-dependent and ubiquitin-independent degradation of fulllength proteins

6. HbYX-dependent $20 S$ gate opening counteracts inhibition by $A \beta^{*} 56$ oligomers

Supplementary Figures

1. $A 11(+)$ soluble aggregates of $A \beta, \alpha-S y n$, and Htt-Q53 impair the mammalian $20 S$ proteasome in a concentration-dependent manner

2. $A 11(+)$ oligomers from $A \beta, \alpha-S y n$, and $H t t-53 Q$ impair substrates that are specific for all three proteolytic sites in the mammalian $20 \mathrm{~S}$ proteasome

3. $A 11(+) A \beta$ intermediate oligomers $(A \beta-i O)$ impair the degradation of substrates specific for each of the three peptidase activities in the 20S, and $A 11(-) A \beta$ protofibrils (A $3-P F)$ do not have any effect

4. Soluble high molecular weight $A \beta$ oligomers also impair the $20 \mathrm{~S}$ proteasome if they are A11 positive.

5. $A 11(+) A \beta$ protofibrils impair the mammalian $20 \mathrm{~S}$ proteasome 
6. Incubation with antibody targeted to the $A \beta N$-terminus (1-8) does not rescue proteasome activity

7. $A \beta^{*} 56$ oligomers bind to the $20 \mathrm{~S}$ proteasome

8. Substrate saturation curve on the WT $20 S$ proteasome with and without $A \beta^{*} 56$ oligomers

\section{Study 2}

Figures

1. Proteasome activators - HbYX motif docking into $20 \mathrm{~S}$ a ring

2. Highly conserved $\alpha$-intersubunit pocket residues are required for PAN-HbYX gate activation

3. HbYX of peptides of different lengths to stimulate gate opening

4. Effect of ZYA derivatives on proteasome gate activation

5. ZYA activates the proteasome gate

6. ZYA activates protein degradation and prevent proteasome impairment by neurodegenerative-disease associated oligomers 


\section{Glossary}

$\begin{array}{ll}\text { A } & \text { amyloid- } \beta \text { peptide } \\ \text { Ac } & \text { N-terminal acetylation } \\ \text { Amc } & \text { 7-Amino-4-methylcoumarin } \\ \text { a-Syn } & \text { a-synuclein } \\ \text { ATPYS } & \text { adenosine 5'-[r-thio]-triphosphate } \\ \text { Boc } & \text { tert-butyloxycarbonyl } \\ \text { CNS } & \text { central nervous system } \\ \text { Ctl } & \text { control } \\ \text { DMSO } & \text { dimethyl sulfoxide } \\ \text { DRG } & \text { dorsal root ganglion } \\ \text { FITC } & \text { fluorescein isothiocyanate } \\ \text { HbYX } & \text { hydrophobic-tyrosine-[any residue] motif } \\ \text { Htt-Q53 } & \text { huntingtin protein exon 1 (53 polyglutamine repeat) } \\ \text { MM } & \text { multiple myeloma } \\ \text { RRMM } & \text { relapsed refractory multiple myeloma } \\ \text { nL } & \text { norleucine } \\ \text { ND } & \text { Neurodegeneration } \\ \text { PA } & \text { Proteasome activator } \\ \text { PN } & \text { peripheral neuropathy } \\ \text { PNS } & \text { peripheral nervous system } \\ \text { RRMM } & \text { relapsed/refractory multiple myeloma } \\ \text { SDS-PAGE } & \text { sodium dodecyl sulfate polyacrylamide gel electrophoresis } \\ \text { Ub } & \text { ubiquitin } \\ \text { UFD } & \text { ubiquitin fusion degradation pathway } \\ \text { UPS } & \text { ubiquitin proteasome system } \\ \text { Z } & \text { carboxybenzyl }\end{array}$




\section{Literature Review - Part 1}

\section{Neurodegenerative disease and the proteasome}

\section{Neurodegenerative disease}

Patients with neurodegenerative disease commonly suffer from escalating dementia, memory loss, and motor impairment as their disease progresses, ultimately resulting in death. The most common, Alzheimer's disease (AD), is the fourth leading cause of death in the elderly and a leading cause of poor health and disability ${ }^{1}$. Deaths due to AD increased by $68 \%$ over the past decade. The annual cost of care for AD is over $\$ 250$ billion dollars, and is projected to reach $\$ 1.2$ trillion by $2050^{1}$. The devastation caused by the development of $A D$ on the individual and the family unit is remarkable. Moreover, if left untreated the expected catastrophic impact of AD on our aging nation has been extensively modeled and discussed ${ }^{2}$. These facts clearly highlight the need for development of therapeutic strategies to combat these costly and deadly diseases.

Compelling evidence from decades of research indicates that diverse neurodegenerative diseases might have a common cause and pathological mechanism protein misfolding, oligomerization, and accumulation, resulting in loss of proteostasis and neuronal death ${ }^{3-5}$. The original amyloid-

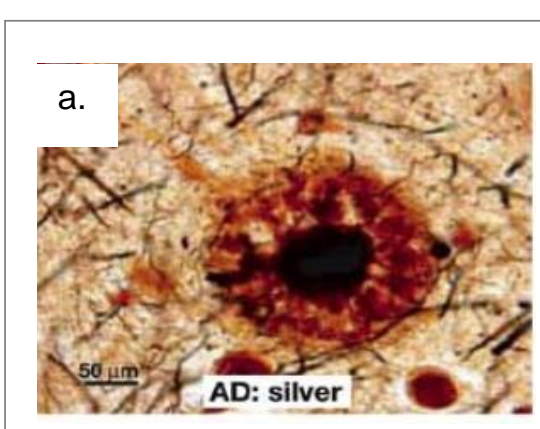

c.

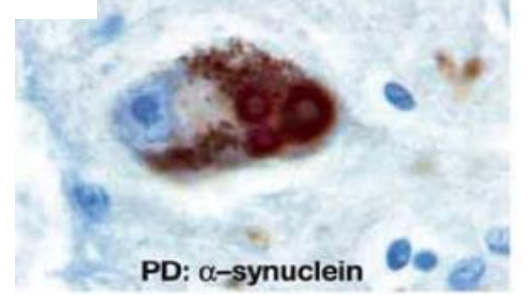

Figure 1. Characteristic neurodegenerative disease neuropathological lesions. (a) AD, neuritic plaque, silver stained (Hirano method)(cerebral cortex). (b) Alzheimer's disease $(A D)$, neuritic plaque labeled for $A \beta$ (cerebral cortex). (c) Parkinson's disease (PD), Lewy bodies labeled for $\alpha$-synuclein (substantial nigra). (d) Huntington's disease (HD), intranuclear inclusion labeled for huntingtin (cerebral cortex). Scale bar $50 \mu \mathrm{m}$. Figure adapted from [11].

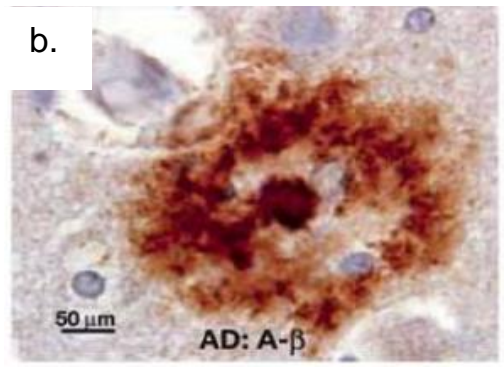

d.

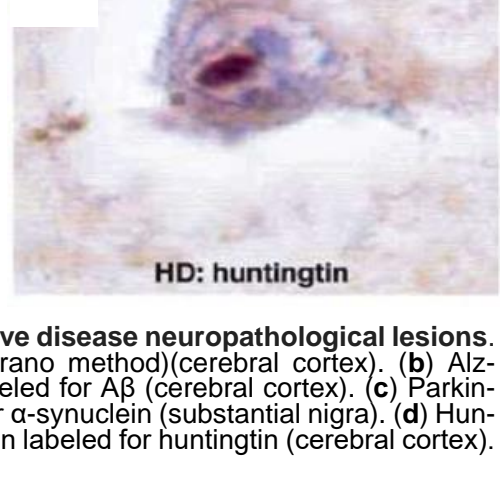




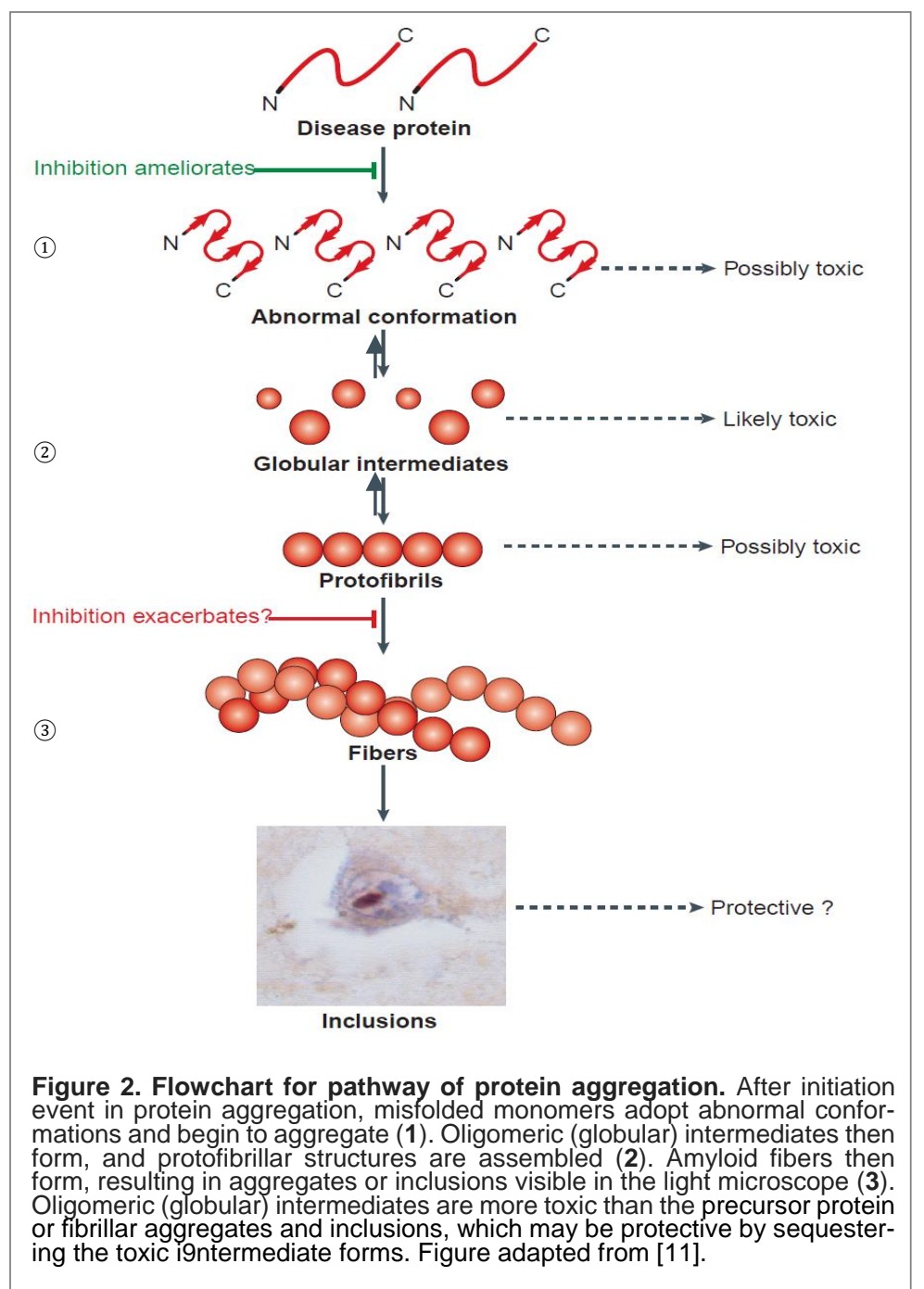

hypothesis postulated the extracellular, insoluble amyloid-fibrils were responsible for neuron death ${ }^{6}$. Early treatments focused on ameliorating pathologies visible with light microscopy (e.g. insoluble protein aggregates: amyloid plaques, intracellular tangles, inclusion bodies) (Figure 1). However, visible pathology alone does not correlate well with disease ${ }^{7-}$ 10 and clinical trials aimed at preventing the formation of or removing existing insoluble protein aggregates have failed ${ }^{11}$ (Figure 2). The most likely explanation is the insoluble aggregates represent an end-stage of the protein aggregation cascade, and that earlier intermediate steps in the cascade are directly tied to disease pathogenesis. In this proposed model, the insoluble protein aggregates may be protective by sequestering the toxic intermediates (Figure 2) ${ }^{12}$. Culminating evidence from the past two decades has revealed that soluble, oligomeric forms of protein aggregates (such as $A \beta$ in Alzheimer's disease, $\alpha$-Synuclein in Parkinson's disease, and mutant huntingtin in Huntington's disease) are key players in proteostasis dysfunction ${ }^{13,14}$. It is now widely appreciated that soluble oligomers of misfolded proteins (e.g. A $\beta$, Alzheimer's; $\alpha-S y n$, Parkinson's; huntingtin with polyglutamine expansion, Huntington's) and loss of proteostasis are the key drivers of disease development and progression ${ }^{15-19}$. 


\section{Proteostasis and the proteasome}

Proteostasis ${ }^{20,21}$ is maintained by several systems in the cell including the ubiquitin-proteasome system (UPS), chaperones, chaperone-mediated autophagy, and macroautophagy 22 (Figure 3), collectively called the Proteostasis Network (PN). If the efficiency of PN declines, misfolded proteins begin to accumulate and aggregate in the cell, which can disrupt normal cellular functions and even cause cell death ${ }^{23}$. Maintaining proteostasis is especially important for neurons due to their complex architecture, long lifespan, and inability to dilute aggregate load by cell division ${ }^{24}$.

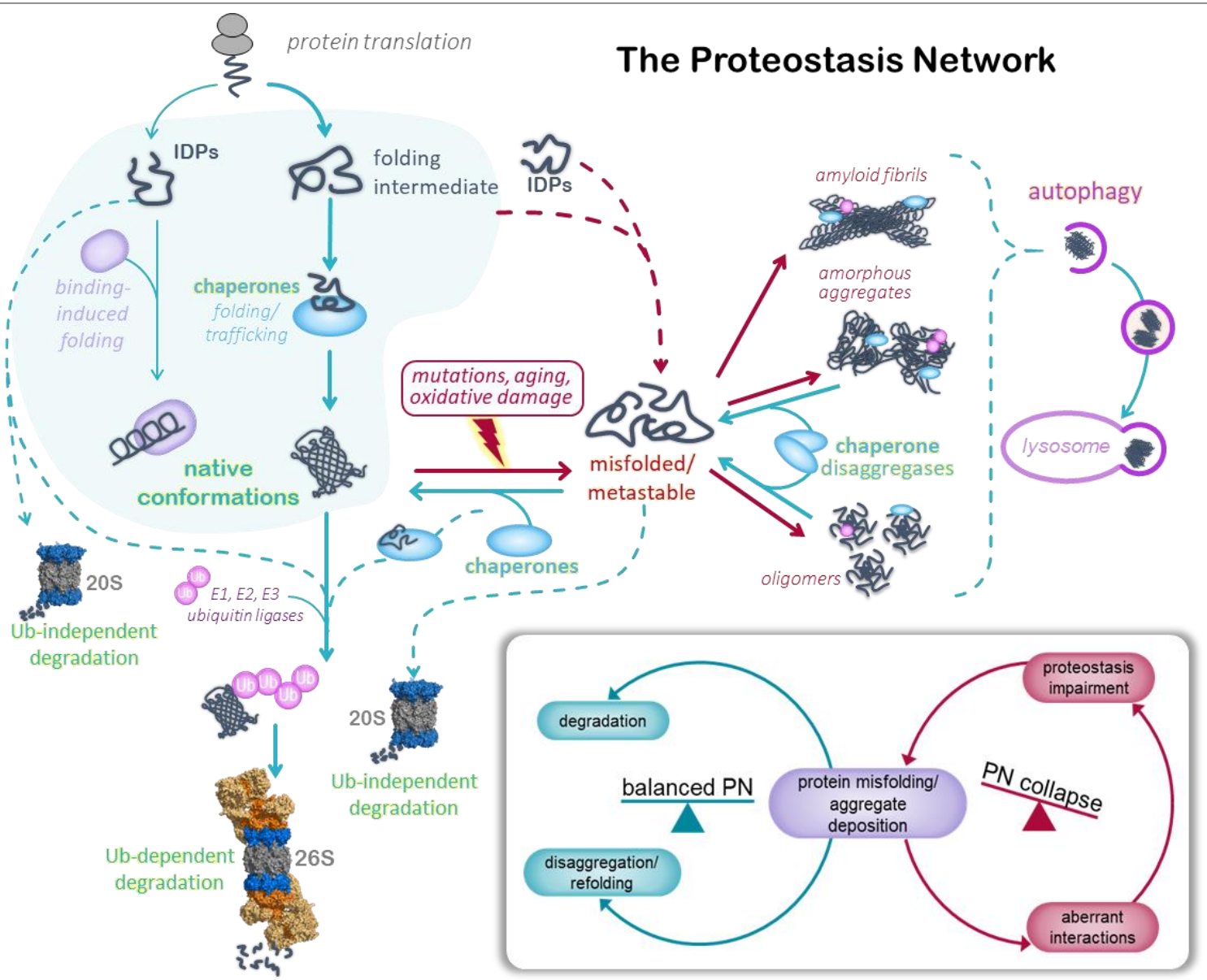

Figure 3. The Proteostasis Network (PN).

Balanced PN: Components of the PN (chaperones (blue spheres), the UPS, and autophagy) work together to maintain proteins in their native conformations and eliminate misfolded or metastable intermediates (blue arrows). On-pathway protein folding is highlighted with a blue background (left). The chaperone network (blue spheres) ensures correct folding and trafficking of nascent proteins. The proteasome degrades over $90 \%$ of the proteome, including intrinsically disordered proteins (IDPs) and folded proteins, and is the principal route for degradation of unneeded, damaged, misfolded or metastable proteins. Cellular stresses (including mutations, post-translational modifications, and oxidative damage) can partially unfold structured proteins (burgundy arrows). Partially unfolded proteins (and IDPS) can be degraded by the 20S and 26S proteasomes, or self-associate to form aggregates (burgundy arrows). In response to accumulation of misfolded proteins and aggregates, the chaperone network facilitates disaggregation and refolding of these proteins or directs them to the proteasome for degradation. Large bulk aggregates resistant to disaggregation are removed by macroautophagy. PN Collapse: When the efficiency of the PN declines, misfolded and metastable proteins accumulate. Protein aggregates cause cellular toxicity, in part through aberrant interactions with cellular proteins, sequestering chaperones, and by impairing the UPS. PN, proteostasis network ; UPS, ubiquitin proteasome system; IDP, intrinsically disordered protein; E1-3, ubiquitin ligases of the ubiquitination conjugation cascade. 
The UPS is the principle route for the degradation of intracellular misfolded, damaged, or unneeded proteins ${ }^{25}$. The UPS is critical for normal functioning of neuronal synapses, including synaptic protein turnover, plasticity, and long-term memory formation, all of which rely on tightly controlled changes in the proteome ${ }^{24,26-28}$. Recently, Ramachandran and Margolis ${ }^{29}$ identified a specialized neuronal membrane proteasome (NMP) that mediates neuronal function by "inside-out" signaling through the production of extracellular proteasome-derived peptides. Pharmacological dissection of the downstream pathways of peptide signaling revealed that NMP-derived peptides act in part by modulating postsynaptic N-methyl-D-aspartate receptors (NMDARs), and it is wellwell-known that NMDARs are critical for neuronal activity-dependent signaling relevant to learning and memory 30,31 .

It is well established that proteasome function is decreased in neurodegenerative diseases, and impaired function has been implicated in the development of many neurodegenerative diseases, including Alzheimer's, Parkinson's, and Huntington's diseases ${ }^{4,32-35}$. Experimental proteasome inhibition in animals recapitulates many as-

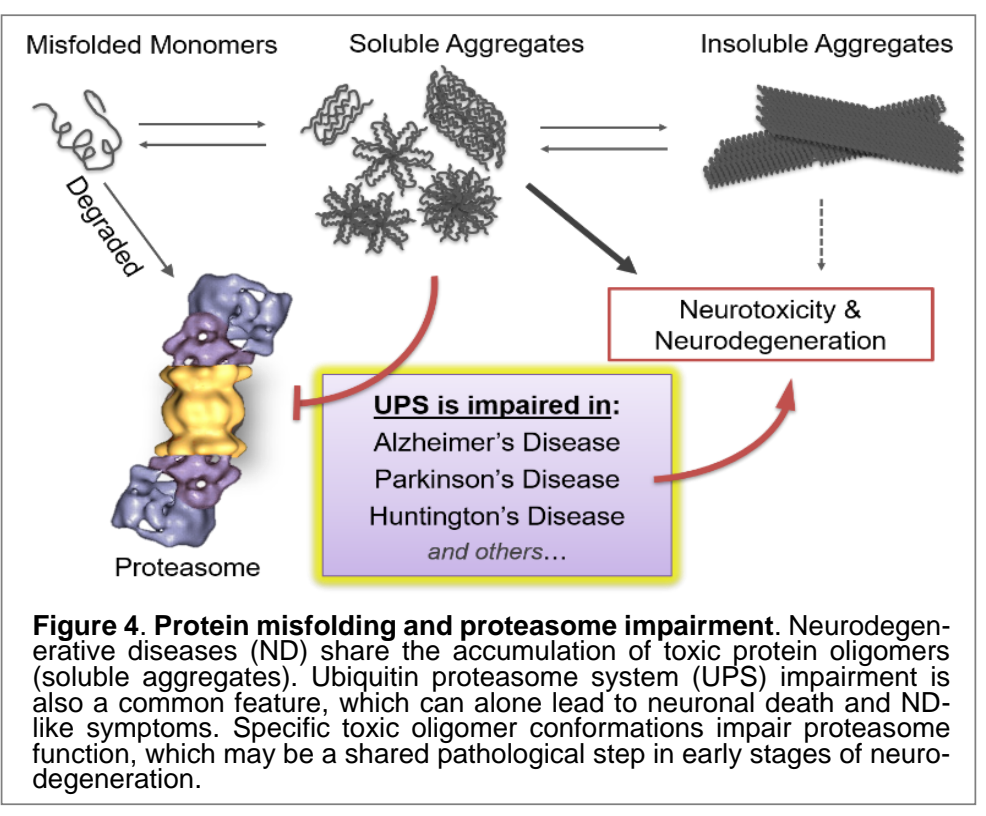
pects of human neurodegenerative diseases ${ }^{36-39}$. For example, stereotaxic unilateral infusion of lactacystin (a selective proteasome inhibitor) into the substantia nigra pars compacta of rats caused neurodegenerative disease like symptoms ${ }^{40}$. Several groups have provided evidence that aggregated proteins from neurodegenerative diseases interact with and impair proteasome ${ }^{41-49}$ (Figure 4). We recently identified a mechanism by which neurodegenerative-disease related 
protein oligomers ( $A \beta, \alpha$-synuclein, and mutant huntingtin) impair the proteasome ${ }^{50}$. Our results provide a novel molecular model for oligomer-driven impairment of proteasome function that is relevant to a variety of neurodegenerative diseases.

Increased proteasome activity shown to be beneficial in many related disease models. Drugs that reverse proteasome impairment in neurodegenerative disease are expected to restore cellular proteostasis, thereby reducing neuronal loss and simultaneously stimulate clearance of toxic oligomers by depleting the pool of misfolded monomers. Such therapeutic interventions have the potential to restore proteostasis in patients suffering from neurodegenerative diseases, as evidenced by several recent studies. For example, Choi et al..$^{51}$ showed that opening of the $20 \mathrm{~S}$ proteasome gate (thereby increasing proteasome activity) in cells leads to enhanced cellular proteasome function, including ubiquitin-dependent protein degradation, decreased protein aggregation, and confers protection from oxidative stress. A better understanding of the proteasome gating functions may lead to the development of novel proteasome gate-activating molecules. 


\section{References}

1. Alzheimer'sAssociation. 2017 Alzheimer's disease facts and figures. Alzheimer's Dement. 13, 325-373 (2017).

2. Holtzman, D. M., Goate, A., Kelly, J. \& Sperling, R. Mapping the road forward in Alzheimer's disease. Sci. Transl. Med. 3, 114ps48 (2011).

3. Brettschneider, J., Tredici, K. D., Lee, V. M. \& Trojanowski, J. Q. Spreading of pathology in neurodegenerative diseases: a focus on human studies. Nat. Rev. Neurosci. 16, 10920 (2015).

4. Rubinsztein, D. C. The roles of intracellular protein-degradation pathways in neurodegeneration. Nature 443, 780-786 (2006).

5. Selkoe, D. J. Folding proteins in fatal ways. Nature 426, 900-904 (2003).

6. Hardy, J. \& Selkoe, D. J. The amyloid hypothesis of Alzheimer's disease: progress and problems on the road to therapeutics. Science 297, 353-356 (2002).

7. Katzman, R. et al. Clinical, pathological, and neurochemical changes in dementia: A subgroup with preserved mental status and numerous neocortical plaques. Ann. Neurol. 23, 138-144 (1988).

8. Terry, R. D. et al. Physical basis of cognitive alterations in alzheimer's disease: Synapse loss is the major correlate of cognitive impairment. Ann. Neurol. 30, 572-580 (1991).

9. McKee, A. C., Kosik, K. S. \& Kowall, N. W. Neuritic pathology and dementia in alzheimer's disease. Ann. Neurol. 30, 156-165 (1991).

10. Dickson, D. W. et al. Identification of normal and pathological aging in prospectively studied nondemented elderly humans. Neurobiol. Aging 13, 179-189 (1992).

11. Rosenblum, W. I. Why Alzheimer trials fail: Removing soluble oligomeric beta amyloid is essential, inconsistent, and difficult. Neurobiology of Aging 35, 969-974 (2014).

12. Ross, C. A. \& Poirier, M. A. Protein aggregation and neurodegenerative disease. Nat. Med. 10, S10 (2004).

13. Haass, C. \& Selkoe, D. J. Soluble protein oligomers in neurodegeneration: Lessons from the Alzheimer's amyloid $\beta$-peptide. Nat. Rev. Mol. Cell Biol. 8, 101-112 (2007).

14. Kayed, R. \& Lasagna-Reeves, C. A. Molecular mechanisms of amyloid oligomers toxicity. Adv. Alzheimer's Dis. 3, 67-78 (2012).

15. Barghorn, S. et al. Globular amyloid $\beta$-peptide1-42 oligomer - A homogenous and stable neuropathological protein in Alzheimer's disease. J. Neurochem. 95, 834-847 (2005).

16. Lue, L.-F. et al. Soluble Amyloid $\beta$ Peptide Concentration as a Predictor of Synaptic Change in Alzheimer's Disease. Am. J. Pathol. 155, 853-862 (1999).

17. McLean, C. A. et al. Soluble pool of $A \beta$ amyloid as a determinant of severity of neurodegeneration in Alzheimer's disease. Ann. Neurol. 46, 860-866 (1999).

18. Stefani, M. Structural features and cytotoxicity of amyloid oligomers: Implications in Alzheimer's disease and other diseases with amyloid deposits. Progress in Neurobiology 99, 226-245 (2012).

19. Caughey, B. \& Lansbury, P. T. Protofibrils, pores, fibrils, and neurodegeneration: Separating the responsible protein aggregates from the innocent bystanders. Annu. Rev. Neurosci. 26, 267-298 (2003).

20. Calamini, B. \& Morimoto, R. I. Protein homeostasis as a therapeutic target for diseases of protein conformation. Curr. Top. Med. Chem. 12, 2623-40 (2012).

21. Labbadia, J. \& Morimoto, R. I. The biology of proteostasis in aging and disease. Annu. Rev. Biochem. 84, 435-464 (2015).

22. Ciechanover, A. Intracellular protein degradation: from a vague idea through the lysosome and the ubiquitin-proteasome system and onto human diseases and drug targeting. Bioorganic Med. Chem. 21, 3400-3410 (2017).

23. Ciechanover, A. \& Kwon, Y. T. Degradation of misfolded proteins in neurodegenerative 
diseases: therapeutic targets and strategies. Exp. Mol. Med. 47, e147 (2015).

24. Tai, H.-C. \& Schuman, E. M. Ubiquitin, the proteasome and protein degradation in neuronal function and dysfunction. Nat. Rev. Neurosci. 9, 826-38 (2008).

25. Glickman, M. H. \& Ciechanover, A. The ubiquitin-proteasome proteolytic pathway: Destruction for the sake of construction. Physiol Rev 82, 373-428 (2002).

26. Fonseca, R., Vabulas, R. M., Hartl, F. U., Bonhoeffer, T. \& Nägerl, U. V. A balance of protein synthesis and proteasome-dependent degradation determines the maintenance of LTP. Neuron 52, 239-245 (2006).

27. Djakovic, S. N. et al. Phosphorylation of Rpt6 regulates synaptic strength in hippocampal neurons. J. Neurosci. 32, 5126-5131 (2012).

28. Aso, E. et al. Amyloid generation and dysfunctional immunoproteasome activation with disease progression in animal model of familial Alzheimer's disease. Brain Pathol. 22, 63653 (2012).

29. Ramachandran, K. V \& Margolis, S. S. A mammalian nervous-system-specific plasma membrane proteasome complex that modulates neuronal function. Nat. Struct. Mol. Biol. 24, 419-430 (2017).

30. Xia, Z., Dudek, H., Miranti, C. K. \& Greenberg, M. E. Calcium influx via the NMDA receptor induces immediate early gene transcription by a MAP kinase/ERK-dependent mechanism. J. Neurosci. 16, 5425-36 (1996).

31. Nicoll, R. A. \& Roche, K. W. Long-term potentiation: Peeling the onion. Neuropharmacology 74, 18-22 (2013).

32. Ciechanover, A. \& Brundin, P. The ubiquitin proteasome system in neurodegenerative diseases: Sometimes the chicken, sometimes the egg. Neuron 40, 427-446 (2003).

33. McNaught, K. S., Olanow, C. W., Halliwell, B., Isacson, O. \& Jenner, P. Failure of the ubiquitin-proteasome system in Parkinson's disease. Nat. Rev. Neurosci. 2, 589-594 (2001).

34. McKinnon, C. \& Tabrizi, S. J. The ubiquitin-proteasome system in neurodegeneration. Antioxid. Redox Signal. 5, 2302-2321 (2014).

35. Ortega, Z., Díaz-Hernández, M. \& Lucas, J. J. Is the ubiquitin-proteasome system impaired in Huntington's disease? Cell. Mol. Life Sci. 64, 2245-2257 (2007).

36. McNaught, K. S. et al. Impairment of the ubiquitin-proteasome system causes dopaminergic cell death and inclusion body formation in ventral mesencephalic cultures. J. Neurochem. 81, 301-306 (2002).

37. McNaught, K. S. P. \& Olanow, C. W. Proteasome inhibitor-induced model of Parkinson's disease. Ann. Neurol. 60, 243-247 (2006).

38. Bedford, L. et al. Depletion of $26 \mathrm{~S}$ proteasomes neurodegeneration and lewy-like inclusions resembling human pale bodies. J. Neurosci. 28, 8189-8198 (2008).

39. $\mathrm{Li}, \mathrm{X}$. et al. Inhibiting the ubiquitin-proteasome system leads to preferential accumulation of toxic N-terminal mutant huntingtin fragments. Hum. Mol. Genet. 19, 2445-2455 (2010).

40. McNaught, K. S. P. et al. Proteasome inhibition causes nigral degeneration with inclusion bodies in rats. Neuroreport 13, 1437-1441 (2002).

41. Cecarini, V. et al. Amyloid peptides in different assembly states and related effects on isolated and cellular proteasomes. Brain Res. 1209, 8-18 (2008).

42. Díaz-Hernández, M. et al. Inhibition of $26 \mathrm{~S}$ proteasome activity by huntingtin filaments but not inclusion bodies isolated from mouse and human brain. J. Neurochem. 98, 1585-1596 (2006).

43. Gregori, L., Fuchs, C., Figueiredo-Pereira, M. E., Van Nostrand, W. E. \& Goldgaber, D. Amyloid beta-protein inhibits ubiquitin-dependent protein degradation in vitro. J. Biol. Chem. 270, 19702-19708 (1995).

44. Lindersson, E. et al. Proteasomal inhibition by a-synuclein filaments and oligomers. J. Biol. Chem. 279, 12924-12934 (2004). 
45. Bence, N. F., Sampat, R. M. \& Kopito, R. R. Impairment of the ubiquitin-proteasome system by protein aggregation. Science 292, 1552-1555 (2001).

46. Oh, S. et al. Amyloid peptide attenuates the proteasome activity in neuronal cells. Mech. Ageing Dev. 126, 1292-1299 (2005).

47. Tanaka, Y. et al. Inducible expression of mutant alpha-synuclein decreases proteasome activity and increases sensitivity to mitochondria-dependent apoptosis. Hum. Mol. Genet. 10, 919-926 (2001).

48. Tseng, B. P., Green, K. N., Chan, J. L., Blurton-jones, M. \& Laferla, F. M. Abeta inhibits the proteasome and enhances amyloid and tau accumulation. Neurobiol. Aging 29, 1607-1618 (2008).

49. Emmanouilidou, E., Stefanis, L. \& Vekrellis, K. Cell-produced a-synuclein oligomers are targeted to, and impair, the $26 \mathrm{~S}$ proteasome. Neurobiol. Aging 31, 953-968 (2010).

50. Thibaudeau, T. A., Anderson, R. T. \& Smith, D. M. A common mechanism of proteasome impairment by neurodegenerative disease-associated oligomers. Nat. Commun. 9, 1097 (2018).

51. Choi, W. H. et al. Open-gate mutants of the mammalian proteasome show enhanced ubiquitin-conjugate degradation. Nat. Commun. 7, 10963 (2016). 


\title{
Literature Review - Part 2
}

\section{A practical review of proteasome pharmacology}

\author{
Tiffany A. Thibaudeau ${ }^{1}$ and David M. Smith ${ }^{1 *}$ \\ Pharmacological Reviews, volume 71, pages 170-197 (2019) \\ DOI: https://doi.org/10.1124/pr.117.015370
}

${ }^{1}$ Program in Biochemistry and Molecular Biology, Department of Biochemistry, West Virginia University, Morgantown, WV

* Corresponding Author: David M. Smith, West Virginia University Department of Biochemistry, P.O. Box 9142, Morgantown WV, 26506. Email: dmsmith@hsc.wvu.edu

Keywords. Proteasome, proteasome gate activators, $\mathrm{HbYX}$ motif

Financial support: NIH-R01GM107129 to D.M.S.

The content is solely the responsibility of the authors and does not necessarily represent the official views of the $\mathrm{NIH}$.

This article is licensed under a Creative Commons Attribution 4.0 International License: https://creativecommons.org/licenses/by/4.0/legalcode. Modified with minor text edits. 


\section{Abstract}

The Ubiquitin Proteasome System (UPS) degrades individual proteins in a highly regulated fashion and is responsible for the degradation of misfolded, damaged, or unneeded cellular proteins. During the past 20 years, investigators have established a critical role for the UPS in essentially every cellular process including: cell cycle progression, transcriptional regulation, genome integrity, apoptosis, immune responses, and neuronal plasticity. At the center of the UPS is the proteasome, a large and complex molecular machine containing a multicatalytic protease complex. When the efficiency of this proteostasis system is perturbed, misfolded and damaged protein aggregates can accumulate to toxic levels and cause neuronal dysfunction, which may underlie many neurodegenerative diseases. In addition, many cancers rely on robust proteasome activity for degrading tumor suppressors and cell cycle checkpoint inhibitors necessary for rapid cell division. Thus clinically, proteasome inhibitors have proven useful to treat some types of cancer, especially Multiple Myeloma. Numerous cellular processes rely on finely tuned proteasome function, making it a crucial target for future therapeutic intervention in many diseases including neurodegenerative diseases, cystic fibrosis, atherosclerosis, autoimmune diseases, diabetes, and cancer. In our review, we discuss the structure and function of the proteasome, the mechanisms of action of different proteasome inhibitors, various techniques to evaluate proteasome function in vitro and in vivo, proteasome inhibitors in preclinical and clinical development, and the feasibility for pharmacological activation of the proteasome to potentially treat neurodegenerative disease. 


\section{Prologue}

Proteasome structure and function

I.A. Proteasome structure and activity

I.A.1. Active sites of the $20 \mathrm{~S}$

I.A.2. Specialized catalytic subunits

I.A.3. Proteasome regulatory caps and their diverse biological roles

I.B. Proteasome dependent cellular processes

I.B.1. Cell cycle

I.B.2. NF-kB activation

I.B.3. Neuronal function

I.B.4. Endoplasmic reticulum-associated protein degradation

II. Development of proteasome inhibitors

II.A. The rise of proteasome inhibitors

II.B. Chemical classes of proteasome inhibitors

II.B.1. Peptide aldehydes

II.B.2. Peptide boronates

II.B.3. Epoxomicin and epoxyketones

II.B.4. Lactacystin and $\beta$-lactone

II.B.5. Vinyl sulfones

II.C. Considerations for proteasome inhibitor design

II.C.1. Substrate binding pockets

II.C.2. Structural analysis of bound inhibitors

II.C.3. Proteasome inhibitor pharmacophore properties

III. Methods for pharmacological proteasome research

III.A. Proteasome purification

II.A.1. Endogenous proteasomes

II.A.2. Affinity-tagged proteasomes

II.A.3. Immunoproteasomes

II.A.4. Validating and storing proteasome preparations

III.B. Monitoring proteasome activity

III.B.1. Peptide-based model substrates

III.B.2. Protein-based model substrates

III.B.3. Proteasome activity in cell culture

III.B.4. In vivo proteasome activity

III.C. Proteasome active site probes

IV. Proteasome inhibitors to treat human disease

IV.A. Hematological malignancies

IV.A.1. Bortezomib and multiple myeloma

IV.A.2. Bortezomib resistance

IV.A.3. Bortezomib dose limiting toxicity

IV.A.4. Bortezomib efficacy in solid tumors

IV.B. Second generation proteasome inhibitors

IV.B.1. Carfilzomib

IV.B.2. Ixazomib

IV.C. Additional proteasome inhibitors in clinical trials

IV.C.1. Marizomib

IV.C.2. Oprozomib

IV.D. Immunoproteasome specific proteasome inhibitors

IV.D.1. ONX-0914

IV.E. Novel combination therapies

V. Novel proteasome pharmacological targets

V.A. Deubiquitinating enzyme inhibitors

V.B. Activation of the $20 \mathrm{~S}$ by gate-opening

Conclusion

Acknowledgements

References 


\section{Prologue}

Early biologists viewed cellular proteins as essentially stable constituents subjected to only minor 'wear and tear'. The widely accepted theory was that dietary proteins functioned primarily as energy, providing fuel for the body. Rudolf Schoenheimer and colleagues challenged that notion in the late 1930s using stable isotopes to show that trace dietary amino acids rapidly incorporated into tissue proteins ${ }^{1}$ and that these proteins are in a dynamic state of synthesis and degradation 2. Today we understand that all intracellular proteins are continually "turning over;" i.e., they are being hydrolyzed into their constituent amino acids and replaced via de novo synthesis. Individual proteins are degraded at different rates; varying from minutes for certain regulatory enzymes, to days for myosin heavy chain in cardiac muscle, to months for hemoglobin in erythrocytes ${ }^{3}$. While cytosolic proteins can be degraded in lysosomes (via chaperone-mediated autophagy and macroautophagy), the majority are degraded by the proteasome ${ }^{4}$.

The discovery of a special class of cytoplasmic granules containing acid hydrolases in the 50's 5,6, called lysosomes ${ }^{7}$, was an important step forward in understanding intracellular protein breakdown. Breakdown of endogenous (autophagy) and exogenous (heterophagy) material was believed to occur in lysosomes, the 'intracellular digestive system' ${ }^{8}$. Because, peptide hydrolysis is an exergonic (i.e. downhill) reaction, the discovery of ATP-dependent protein breakdown in mammalian ${ }^{9}$ and bacterial cells ${ }^{10}$ was unexpected. Simpson et al. suggested "the possible existence of two (or more) mechanisms of protein breakdown, one hydrolytic, the other energyrequiring" 9 . Subsequent work over the next two decades firmly established that both rates of protein synthesis and degradation determine the cellular protein concentration as well as the wide variability of protein half-lives ${ }^{11-13}$.

Studies in the 1970's supported the prediction of a new selective degradation pathway that accounted for the wide distribution of protein half-lives ${ }^{14-17}$. Interestingly, cytosolic proteins 
synthesized with structural analogues of a normal amino acids are rapidly degraded within the cell $14,18,19$. These seminal observations added another layer of selectivity in which the inherent stability of each protein also determines the degradation rate, presumably to prevent the accumulation of abnormal proteins ${ }^{19}$. However, the mechanism of selectivity remained a mystery. ATP was found to be essential for protein catabolism but it was unknown whether a proteolytic step was directly dependent on ATP or whether it required some additional reactions ${ }^{20}$. Selective and ATP-dependent protein degradation was not congruent with the notion of the lysosome as the key player in protein breakdown. What could be responsible for this exquisitely controlled protein degradation? In 1977 Etlinger and Goldberg identified a novel, soluble, ATP-dependent proteolytic system that was independent from the lysosome ${ }^{21}$. The importance of the soluble degradation system was emphasized when Rechsteiner and colleagues showed that most intracellular proteins are degraded in the cytosol, not the lysosome ${ }^{22}$.

Wilk and Orlowski (1980) purified a 700 kilodalton (kDa) 'multicatalytic proteinase complex' (later shown to be the $20 S$ proteasome) ${ }^{23}$. Unlike all other known proteases, this new protease complex could cleave peptides after basic, acidic, or hydrophobic residues, suggesting that it contained multiple distinct active sites ${ }^{23,24}$. Electron micrographs revealed the complex to be a $700 \mathrm{kDa}$ stacked 'donut' ring structure ${ }^{25}$. Due to their critical roles in intracellular protein breakdown, these protease complexes were collectively renamed 'proteasomes' ${ }^{26}$. Analogous protease complexes of equivalent size, shape, polypeptide composition, and proteolytic activities have since been identified across all three domains of life ${ }^{25,27}$.

The next major advancement in the field came with the discovery of ubiquitin (Ub), a small $\sim 8$ kDa protein with a big role in protein degradation. Aaron Ciechanover and colleagues identified a small heat-stable protein, ubiquitin, covalently conjugated to target substrates ${ }^{28}$, in an ATPdependent manner ${ }^{29,30}$. This led to the proposed model in which protein-substrate modification by several ubiquitin moieties targets it for degradation by a downstream, as-yet unidentified 
protease that cannot recognize the unmodified substrate ${ }^{31}$. It was later shown that some nonubiquitin proteins are also degraded in an ATP-dependent manner ${ }^{32}$. Rechsteiner's group later went on to purify the ATP-dependent 26S proteasome responsible for ubiquitin conjugate degradation ${ }^{33,34}$.

Avram Hershko, Aaron Ciechanover, and Irwin Rose characterized the system of Ub conjugation and its role in marking proteins for degradation ${ }^{35}$ - an achievement that earned them the Nobel Prize in Chemistry (2004) ${ }^{36}$. Attachment of poly-Ub chains to specific proteins selects them for proteasome-mediated degradation (Figure 1A). Targeting proteins for degradation requires three enzymatic components to link chains of Ub onto selected protein substrates. E1 (Ub-activating enzyme) and E2s (Ub-carrier or conjugating proteins) prepare Ub for conjugation. The E3 (Ubprotein ligase) enzymes control substrate specificity, recognizing substrate degradation signals and catalyzing the transfer of activated $\mathrm{Ub}$ to the substrate ${ }^{35,37}$. Eukaryotic cells contain hundreds of E3 ligases allowing the cell to precisely control ubiquitination and degradation of individual proteins ${ }^{38}$. Ubiquitin conjugation is necessary for cell viability ${ }^{39,40}$ and activity of the ubiquitin pathway is greatly increased in cells making abnormal proteins ${ }^{41}$.

During the past 20 years, investigators have established the critical role of the UPS in cell cycle progression ${ }^{42-44}$, transcriptional regulation, genome integrity, apoptosis, immune responses ${ }^{45}$, and the pathogenesis of many human diseases ${ }^{4,46}$. With respect to disease, the proteasome is particularly important for maintaining cellular protein homeostasis (i.e. proteostasis). When the efficiency of proteostasis systems decline, misfolded and damaged proteins aggregate to toxic levels within the cell, potentially giving rise to many neurodegenerative diseases 47,48 . Too much of a good thing can be just as detrimental. Cancer cells rely on robust proteasome activity for degrading tumor suppressors and cell cycle checkpoint inhibitors necessary for rapid cell division 49. Numerous processes rely on finely tuned proteasome function, making it a crucial target for therapeutic intervention in many diseases including neurodegenerative diseases, cystic fibrosis, 
atherosclerosis, autoimmune diseases, diabetes, and cancer ${ }^{50}$. In 2003 bortezomib (Velcade®) became the first US Food and Drug Administration (FDA) approved proteasome inhibitor as a third line treatment for multiple myeloma (MM).

In this review, we first discuss the structure, function, and regulation of the proteasome. Then we discuss the classes and mechanisms of action of Proteasome Inhibitors (PIs). Next, we summarize commonly used in vitro and in vivo techniques for studying proteasome activity and inhibition followed by a review of currently FDA approved proteasome inhibitors (PIs) as well as novel inhibitors undergoing clinical and preclinical trials. Lastly, we discuss how pharmacological activation of the proteasome- could produce novel therapeutics to treat neurodegenerative disease.

I. Proteasome structure and function. Overview of the current understanding of 20S/26S proteasome structure, mechanism of protein degradation, and the cellular processes associated with proteasome impairment or hyperactivation in human diseases.

II. Development of proteasome inhibitors. Proteasome inhibitor classes and mechanisms of action, mechanisms of proteasome inhibitor resistance, and considerations for proteasome inhibitor design.

III. Methods for pharmacological proteasome research. Overview of in vitro and in vivo methods for investigating proteasome activity

IV. Proteasome inhibitors to treat human disease. Review FDA approved proteasome inhibitors, clinical trials, and pre-clinical research to treat cancer and inflammatory disease, including strategies aimed at overcoming proteasome inhibitor resistance.

V. Novel proteasome pharmacological targets. Novel proteasome targets for modulating proteasome activity. 


\section{Proteasome Structure and Function}

\section{I.A. Proteasome structure and activity}

The $26 \mathrm{~S}$ proteasome is a 2.4 megadalton (MDa) molecular machine that makes up nearly $2 \%$ of total cellular protein ${ }^{51}$. It is composed of a $20 \mathrm{~S}$ proteasome core particle capped on one or both ends by the $19 \mathrm{~S}$ regulatory particle (Figure 1B). It degrades proteins by a multistep process; the $19 S$ regulatory particle binds ubiquitinated substrates, opens a substrate entry gate in the $20 \mathrm{~S}$ 52,53 , and unfolds its substrates by linearly translocating them into the $20 \mathrm{~S}$ catalytic chamber where

they are degraded to peptides ${ }^{54,55}$. Numerous studies over the past two decades have developed our present understanding of proteasome structure and function. The first 20S core particle was crystalized in the late 90 's ${ }^{56}$. Since then hundreds of $20 S$ structures, complexed with regulators or inhibitors, have been solved.

Eukaryotic proteasomes contain four stacked heteroheptameric rings arranged in an $\alpha_{7}-\beta_{7}-\beta_{7}-\alpha_{7}$ fashion ${ }^{56}$. The amino(N)-termini of the a subunits form a "gate," folding over the 13 Angstrom central pore and occluding access to the proteolytic sites located on the $\beta$ subunit lumen ${ }^{57}$. Passage through this gate is the rate limiting step and prevents unregulated protein degradation 58. The $\alpha \mathrm{N}$-termini tails are highly conserved, containing a tyrosine-aspartate-arginine (YDR) motif that forms salt bridges with neighboring tails that obstruct the $13 \mathrm{~A}$ entry pore. The $\alpha 3 \mathrm{~N}$-terminus is the lynchpin, critical for stabilizing the closed gate confirmation ${ }^{58}$. Note that the purified latent $20 S$ proteasome still exhibits a degree of peptidase activity due to stochastic conformational fluctuations within the $\mathrm{N}$-termini ${ }^{59,60}$. Interestingly, deletion of the first eight $\alpha 3$ residues $(\alpha 3 \Delta \mathrm{N}$ 20S) sufficiently destabilizes the closed-gate conformation and accelerates the entry and degradation of peptides ${ }^{58}$. $\alpha 3 \Delta \mathrm{N}-20 \mathrm{~S}$ crystallographic structures show that the remaining $\mathrm{N}$ termini are disordered resulting in a constitutively open gate ${ }^{58}$. Wild type $20 \mathrm{~S}$ proteasome activity is similarly accelerated when bound to a proteasome activator (e.g. 19S/PA700, 11S/PA28, 
BIm10/PA200) ${ }^{61}$. Proteasome activators bind to a free end of the a subunit ring and "open" the gate by distinct mechanisms that are discussed in detail below.

I.A.1. Active sites of the 20S. The eukaryotic $20 \mathrm{~S}$ proteasome contains six proteolytically active $\beta$-subunits, three on each $\beta$-ring that exhibit different substrate preferences (Figure $2 \mathrm{~A}$ and $2 \mathrm{~B}$ ). The various substrate binding pockets determine active site specificity like classical proteases but with more complexities. The binding pockets themselves are formed by specific interactions between the catalytic subunit and the neighboring $\beta$-subunit ${ }^{62}$. As a result, the proteasome is not simply a complex of independent proteases but is a unique multicatalytic enzyme functioning only when wholly intact. The chymotrypsin-like site $(\beta 5)$ preferentially cleaves after hydrophobic residues, the trypsin-like site $(\beta 2)$ preferentially cleaves after basic residues, and the caspase-like site ( $\beta 1)$ preferentially cleaves after acidic residues ${ }^{55,63}$. Despite their names, these sites do not share the catalytic mechanisms of their namesakes and their substrate preferences are much broader than the names imply ${ }^{51}$. Multiple catalytic sites with varying specificities advantageously allow for the rapid and processive degradation of cellular proteins.

All proteasome active sites utilize an $\mathrm{N}$-terminal threonine nucleophile. Enzyme inhibitor and sitedirected mutagenesis studies compose much of what we know about the proteasome's unusual catalytic mechanism ${ }^{64}$. Although lacking the classical triad of cysteine and serine proteases, proteasome sensitivity to peptide aldehyde inhibitors suggests a similar catalytic mechanism ${ }^{64}$. Accordingly, the crystal structure of the $20 \mathrm{~S}$ bound to the peptide aldehyde Ac-Leu-Leu-nLeu-al (ALLN) reveals a hemiacetyl bond between the $\beta$ subunit $\mathrm{N}$-terminal threonine hydroxyl groups 64. Proteasome inhibitors (lactacystin, vinyl sulfones, and epoxyketones) are often found to covalently modify this threonine. As expected, mutation to a serine residue retains significant activity while mutation to an alanine residue completely abolishes activity ${ }^{64}$. 
A. Substrate ubiquitination

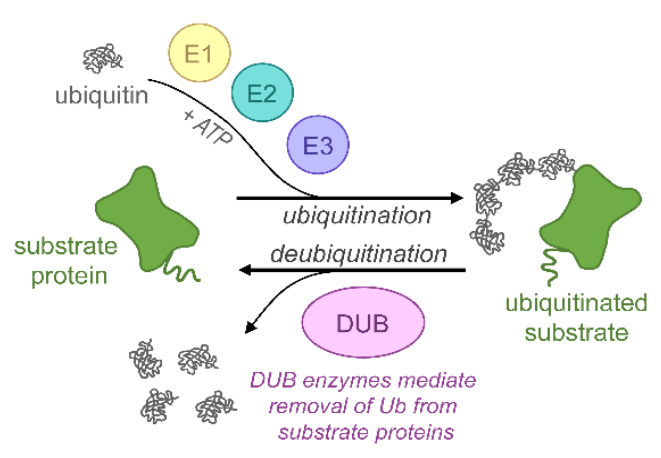

B. Substrate processing by the 265 proteasome

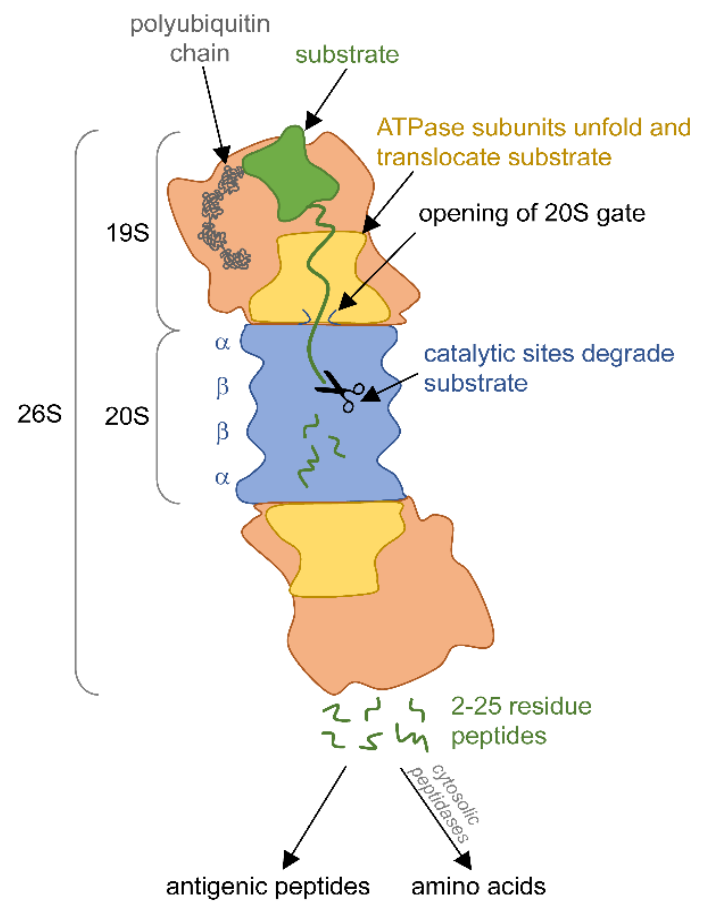

Figure 1. The ubiquitin proteasome pathway

A. Simplified model of the ubiquitin conjugation system. B. Primary steps involved in ubiquitinated-substrate processing by the $26 \mathrm{~S}$ proteasome.

1.A.2. Specialized catalytic subunits. Some cell types express $\beta$ subunits with modified catalytic sites under certain conditions. Immune cells constitutively express alternative catalytic subunits $(\beta 1 \mathrm{i} / \mathrm{LMP} 2, \beta 2 \mathrm{i} / \mathrm{MECL}-1$, and $\beta 5 \mathrm{i} / \mathrm{LMP} 7)$ that are preferentially incorporated into the $20 \mathrm{~S}$ proteasome de novo in place of the constitutive $\beta 1(\beta 1 c), \beta 2(\beta 2 c)$, and $\beta 5(\beta 5 c)$ subunits, forming the immunoproteasome ${ }^{65}$ (Figure 2A). The immunoproteasome is also expressed in nonhematopoietic cells when exposed to interferon (INF)- $y$ or tumor necrosis factor (TNF)- $\alpha{ }^{65}$. To our best knowledge the main purpose of the immunoproteasome is to enhance ligand generation for major histocompatibility complex class I (MHC-I) molecules 66 that allow for immune surveillance ${ }^{67}$. How do these immune subunits do this? These subunits use the same catalytic mechanism as their constitutively expressed counterparts but they have different cleavage preferences due to changes in substrate binding pockets ${ }^{64,68}$. The most striking 
difference between constitutive and immunoproteasomes is the $\beta 1 \mathrm{i}$ subunit, which lacks caspaselike activity but instead cleaves after hydrophobic residues ${ }^{64}$. This is a crucial difference because only MHC-I molecules with tightly bound ligands are expressed on the cell surface. Tight class-I binding requires ligands 8-9 amino acids in length with either a hydrophobic or basic anchor residue at the carboxy $(\mathrm{C})$-terminus. Ligands with acidic $\mathrm{C}$-termini are not accepted ${ }^{69,70}$. Thus, the immunoproteasome facilitates the production of peptides suitable for MHC-I presentation ${ }^{66}$.

Human thymus cortical epithelial cells express a thymic-specific catalytic subunit $\beta 5 t$, which together with $\beta 1 \mathrm{i}$ and $\beta 2 \mathrm{i}$ form the thymoproteasome ${ }^{71}$ (Figure $1 \mathrm{~A}$ ). The thymoproteasome is essential for $T$ lymphocytes positive selection. Compared to $\beta 5 \mathrm{c}$ and $\beta 5 \mathrm{i}$, the $\beta 5 \mathrm{t}$ has weak chymotrypsin-like activity, thus it is speculated that thymoproteasomes facilitate the low affinity MHC-I molecule ligand production necessary for positive selection ${ }^{71}$. Further details on unique functions of tissue-specific proteasomes can be found in Kniepert \& Groettrup (2014) ${ }^{70}$.

1.A.3. Proteasome regulatory caps and their diverse biological roles. Regulation of gate opening in the $20 \mathrm{~S}$ proteasome is an important aspect of proteasome function, and as such the cell has evolved many different proteasomal regulators that control $20 S$ gate-opening ${ }^{61}$. The most well-known regulator is the 195 (also known as PA700), a component of the 26S proteasome. The $26 \mathrm{~S}$ proteasome is a structurally dynamic complex, adopting large-scale conformational changes around the central axis during the ATP-dependent processing of substrates ${ }^{72-75}$. These structural changes appear to be necessary for substrate protein unfolding and injection into the $20 \mathrm{~S}$ core particle.

Ubiquitin-dependent degradation requires several steps: (1) substrate binding and commitment, (2) $20 \mathrm{~S}$ gate opening, (3) substrate unfolding and translocation, and (4) deubiquitination (Figure 2B). (1) The 19S regulatory particle has three integral subunits that serve as substrate receptors: Rpn1, Rpn10, and Rpn13. These substrate receptors reversibly associate with Ub, and have only 
A.

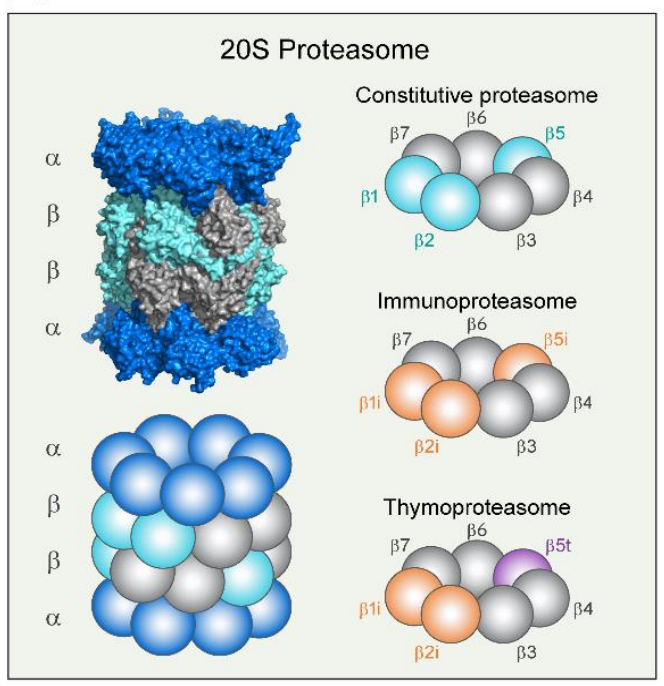

C.

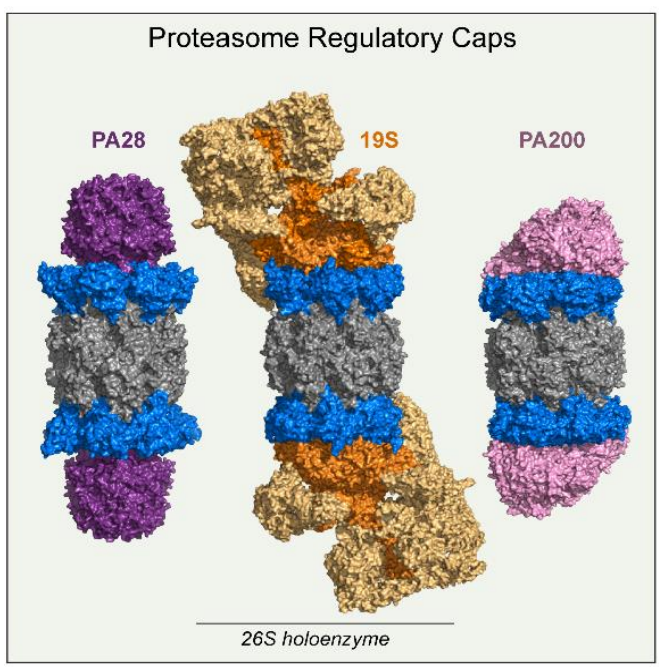

B.

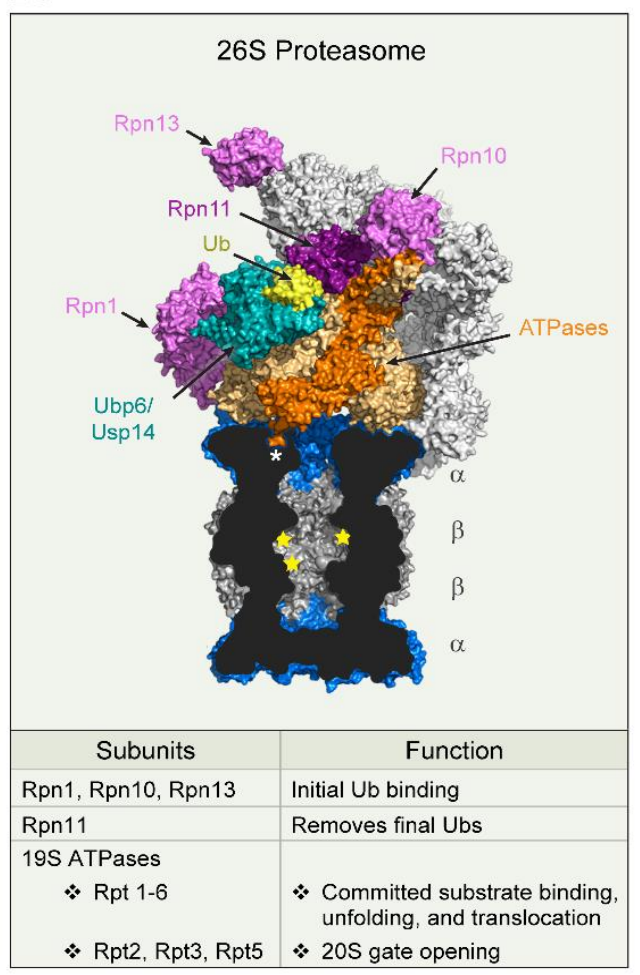

Figure 2. Proteasome structure and function.

A. Structures (PDB 4r3o) and cartoon representation of $20 \mathrm{~S}$ proteasome, highlighting the different $\beta$-subunit combinations found in tissue specific proteasomes discuss in the text. B. Structure of $26 \mathrm{~S}$ proteasome in complex with Ubp6 (PDB 5a5b). Cross section of 20S proteasome reveals the C-terminus of Rpt5 ATPase (dark orange) positioned in the inter- $\alpha$-subunit pocket $\left(^{*}\right)$. Proteolytic sites are marked by yellow stars. Labeled 19S subunits are discussed in the text. C. 20 S proteasomes (blue and gray) complexed with regulatory caps: PA28 homolog PA26 (PDB 1fnt), 19S (PDB 5gjr), and PA200 yeast homolog BIm10 (PDB 4v7o). 19S ATPases are dark orange, non-ATPase subunits are light orange.

low affinity for mono- $\mathrm{Ub}{ }^{76}$. The multiplicity of $\mathrm{Ub}$ receptors coupled with a variety of shuttling factors (e.g. proteins that have a ubiquitin-like domain (UBL) and a ubiquitin associating domain (UBA)) allows the 26S proteasome to recognize and degrade many types of Ub conjugates ${ }^{77}$. Substrate binding to the $\mathrm{Ub}$ receptors induces a conformational change aligning the 19S ATPase 
translocation channel directly over the $20 \mathrm{~S}_{\text {gate }}{ }^{73}$, induces gate opening, and stimulates ATP hydrolysis ${ }^{78,79}$. Substrate commitment requires a loosely folded region of the protein (e.g. unstructured initiation site) to insert into the ATPase ring where tyrosine pore loops inside the ATPase ring "grip" the substrate in an ATP-dependent manner ${ }^{80}$. (2) The six ATPase subunits (Rpt1-6) form a ring at the bottom of the 19S complex with their C-termini inserting into the 20S $\alpha$ subunit intersubunit pockets ${ }^{82}$. $19 \mathrm{~S}$-dependent gate-opening requires ATPase C-terminal $\mathrm{HbYX}$ motif binding to intersubunit pockets (between the $\alpha$ subunits) on top of the $20{ }^{83}$. Binding of the $19 S \mathrm{C}$-termini to the $20 \mathrm{~S}$ is thought to induce a conformational change in the $\alpha$ subunits, opening the gate ${ }^{82}$. The exact mechanisms behind the $26 \mathrm{~S} \mathrm{HbYX-motif} \mathrm{gate} \mathrm{opening} \mathrm{in} \mathrm{human} 26 \mathrm{~S}$ proteasomes are not clear. However, binding of a HbYX-motif peptide (the last 8 residues of Rpt5) is sufficient to allosterically induce conformational changes in the $20 S \alpha$-subunit and open the gate ${ }^{84}$. (3) The six ATPase subunits power processive unfolding and translocation of substrates into the $20 \mathrm{~S}$ core coupled with ATP hydrolysis ${ }^{85,86}$. (4) Rpn11 is the integral proteasomesassociated deubiquitinase (DUB) enzyme of the 19 S complex. Rpn11 is positioned directly above the translocation channel when substrate is committed for degradation and removes the entire Ub chain as proteins are translocated ${ }^{76}$. Two other DUBs are transiently associated with proteasomes (Usp14 and Uch37) and they can trim substrate Ub chains prior to the committed step, rescuing the substrate from degradation ${ }^{76}$. Proteasome associated DUBs are discussed in Part V.

In addition to the 19S, there are two other proteasome gate activator families, the $11 \mathrm{~S}$ and PA200/BIm10, neither of which contain unfoldase activity or require ATP (Figure 2C). Higher eukaryotes express three $11 \mathrm{~S}$ regulatory subunits, PA28 $\alpha, \beta, Y$ (aka REG $\alpha, \beta, \gamma$ ) ${ }^{87,88}$. PA28a $\beta$ forms inducible heteroheptamer that is primarily located in the cytoplasm. In contrast, PA28y forms a homoheptamer that is constitutively expressed in the nucleus ${ }^{61,89}$. The biological roles of these regulators remain relatively mysterious. However, both forms of PA28 regulators show 
increased expression following acute oxidative stress in cells, suggesting both play a significant role in oxidized protein degradation ${ }^{90}$. Additionally, IFN- $\gamma$ increases PA28a $\beta$ expression, oxidized protein degradation capacity ${ }^{90}$, and MHC-I ligand generation.

PA200 (BIm10 in yeast) plays a role in spermatogenesis ${ }^{91}$, response to DNA repair ${ }^{92}$, glutamine homeostasis ${ }^{93}$, and mitochondrial inheritance ${ }^{94}$, although molecular details behind many of these functions are not clear. The crystal structure of yeast BIm10-20S shows that BIm10 forms a large HEAT repeat-like solenoid in a 1.5 super helical turn, forming a dome that caps the ends of the $20 \mathrm{~S}$ proteasome ${ }^{94}$. One $\mathrm{C}$-terminal $\mathrm{HbYX}$ motif binds between the $\alpha 5$ and $\alpha 6$ intersubunit pocket and facilitates gate opening ${ }^{95,96}$. As with the $19 \mathrm{~S}$ Rpt5 subunit, an eight-amino acid BIm10 Cterminal fragment (Blm-pep) induces gate opening in purified 20S proteasomes ${ }^{97}$. The BIm-pep bound $20 \mathrm{~S}$ crystal structure closely resembles the bound $\mathrm{HbYX}$ in the full-length BIm10 structure. PA200/BIm10 containing proteasomes specifically catalyze the acetylation-dependent, but not polyubiquitination-dependent, core histone degradation during somatic DNA damage response and spermatogenesis ${ }^{98}$. During spermatogenesis, the spermatoproteasome (formed by PA200, 20S, $\beta 1 \mathrm{i}, \beta 2 \mathrm{i}, \beta 5 \mathrm{i}$ and the human testis specific $\alpha 4_{s}$ ) degrades core histones and is required for

proper sperm maturation and viability ${ }^{98}$. The existence of such proteasomes highlights the seemingly infinite complexities of the cell's repertoire of proteasome complexes for specific cellular roles.

\section{I.B. Proteasome dependent cellular processes}

Proteasome function is essential to cellular homeostasis. In addition to maintaining proteostasis, the proteasome plays a key role in the regulating many physiological processes. Four major areas not previously discussed include cell cycle regulation, NF-KB activation, neuronal function, and ER-associated protein degradation (Figure 3). 


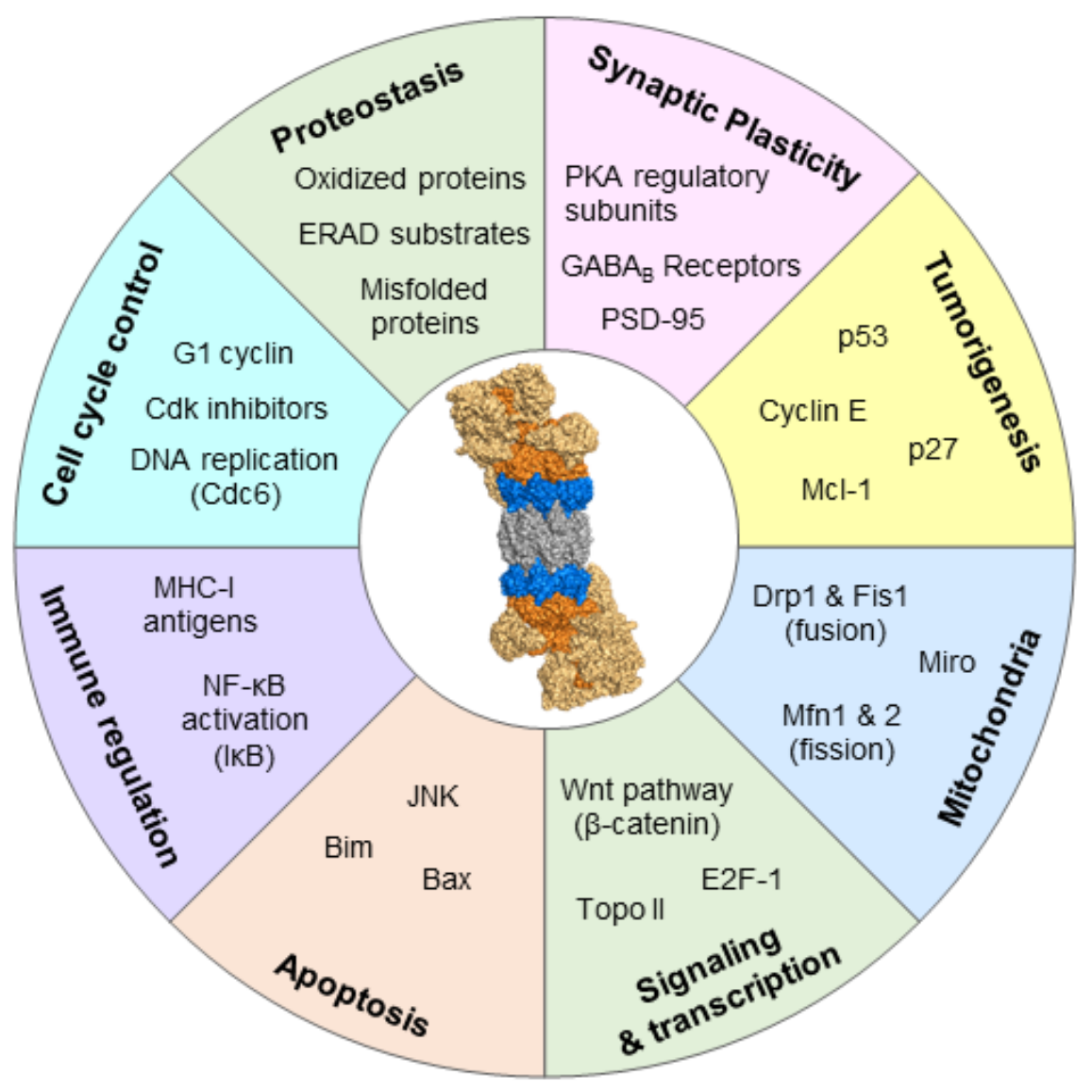

Figure 3. Examples of cellular functions that depend on proteasome function. Important pathways dependent on proteasome function and exemplar substrates.

I.B.1. Cell Cycle. The proteasome degrades many cell cycle regulatory proteins that typically have short half-lives (e.g. cyclin B1, p21, p27) and tumor suppressors (e.g. p53) promoting cycle progression ${ }^{99-103}$. Not surprisingly, most cancers heavily rely on proteasome activity and are more susceptible to proteasome inhibition than normal cells ${ }^{104-106}$. Proteasome inhibition in cancer is discussed in Part III.

I.B.2. NF-kB Activation. The NF-kB transcription factors (NF-kB and Rel proteins) regulate expression of genes involved in innate and adaptive immunity, inflammation, stress responses, B cell development, and lymphoid organogenesis. In cancer cells, NF-kB is critically involved in the expression of the antiapoptotic IAP family of genes as well as BCL-2 pro-survival genes ${ }^{107-109}$. 
Canonical and a non-canonical NF-kB activation requires proteasome-mediated degradation of regulatory elements for transcriptional activation. In the unstimulated state, the inhibitory $\mathrm{IKB}$ subunits bind and sequester NF-KB/Rel complexes in the cytoplasm ${ }^{110}$. In canonical pathway activation, proinflammmatory cytokines activate the IKK complex (IKK , IKKa, and NEMO) which phosphorylates $1 \mathrm{kB}$, leading to IKB ubiquitination and proteasomal degradation ${ }^{111-114}$, freeing NF$\mathrm{kB} /$ RelA complexes. Freed NF-kB/RelA complexes translocate to the nucleus where they (either alone in or combination with other transcription factors) induce target gene expression. In the noncanonical pathway, NF-kB-p100/RelB complexes are inactive in the cytoplasm. Signaling activates the kinase NIK, which activates IKKa complexes that phosphorylate NF-KB2-p100 Cterminal residues. Phosphorylated NFKB is ubiquitinated and processed by the proteasome into NF-kB2-p52, which is transcriptionally competent. Such processing by the proteasome is remarkable since it requires the initiation of protein degradation, followed by termination of degradation at a specific domain, demonstrating exquisite control over degradation. After processing, NF-kB2-p52 translocates to the nucleus and induces target gene expression. NF-kB is a pro-survival pathway and is upregulated in many cancers and inflammatory diseases ${ }^{115}$. Given the indispensable role of proteasome function in activating this pathway, proteasome inhibition is a valid therapeutic target. The role of NF-KB in cancer pathology is discussed further in Part IV.

I.B.3. Neuronal function. Maintaining proteostasis in neurons is especially important due to their complex architecture, long lifespan, and inability to dilute aggregate load through cell division ${ }^{116}$. Importantly, the UPS is critical for normal functioning of neuronal synapses, including synaptic protein turnover, plasticity, and long-term memory formation, which rely on tightly controlled changes in the proteome ${ }^{116-119}$. In addition to the intracellular proteasomes, Ramachandran and Margolis (2017) identified a mammalian nervous-system-specific membrane-associated proteasome complex that rapidly modulates neuronal function ${ }^{120}$. This proteasome complex 
degrades intracellular proteins and releases the products into the synaptic cleft where they stimulate postsynaptic N-methyl-D-aspartate receptor-dependent neuronal signaling, a process important for regulating synaptic function.

The accumulation of aggregation-prone proteins is a hallmark of neurodegenerative disease commonly accompanied by loss of proteostasis and progressive death of neurons ${ }^{121-123}$. It is established that proteasome function is decreased in neurodegenerative diseases, and its impairment is implicated in the development of many neurodegenerative diseases including Alzheimer's, Parkinson's, and Huntington's diseases 122,124-127. To highlight this point, brain region-specific proteasome inhibition closely mirrors the neuropathology and clinical hallmarks of neurodegenerative diseases ${ }^{128-131}$. The importance of targeting the proteasome for potential neurodegenerative disease therapy is discussed in Part V.

\section{I.B.4. Endoplasmic reticulum-associated protein degradation and the unfolded protein}

response. Secretory proteins and most integral membrane proteins are synthesized and enter the endoplasmic reticulum (ER) lumen for proper folding and covalent modifications. The ERassociated protein degradation (ERAD) pathway is an evolutionarily conserved process that discards misfolded ER proteins ${ }^{132}$. Three different ERAD pathways (ERAD-L, $-\mathrm{M}$, and $-\mathrm{C}$ ) are utilized for degrading misfolded ER proteins, depending on whether their misfolded domain is localized in the ER lumen, within the membrane, or on the cytosolic side of the ER membrane, respectively ${ }^{133-135}$. A fourth pathway is responsible for misfolded protein removal from the inner nuclear membrane ${ }^{136,137}$. Each pathway involves distinct Ub ligases and co-factors, although it is unclear how proteins are targets to each pathway. ERAD substrates from all pathways are retrotranslocated to the cytosolic side of the membrane ${ }^{132}$. With the help of ubiquitination machinery and the Cdc48/p97 ATPase complex, the substrates are extracted from the membrane and delivered to the $26 \mathrm{~S}$ proteasome for degradation ${ }^{138-141}$. Proteasome inhibition stalls ERAD and causes misfolded proteins to accumulate within the ER. In response, the cell activates a 
highly conserved signaling pathway called the unfolded protein response (UPR) ${ }^{142}$. Multiple physiological conditions also lead to accumulation of misfolded proteins in the ER and subsequent UPR activation, including hypoxia, glucose deprivation, oxidative stress, and mutations in certain secretory proteins ${ }^{142}$. UPR activation regulates the gene expression involved in protein folding (e.g. chaperones) and ERAD, and decreases protein translation into the ER in an attempt to restore ER homeostasis ${ }^{143}$. The UPR initially performs a protective role in the cell. However, prolonged ER stress and UPR activation eventually leads to cell death ${ }^{143}$. Wu and Rapoport (2018) recently published an extensive review discussing the molecular mechanisms of ERAD and associated protein degradation ${ }^{132}$.

\section{Development of Proteasome Inhibitors (PIs)}

\section{II.A. The Rise of Proteasome Inhibitors}

Our understanding of the importance of the UPS for biological functions and processes rapidly advanced with the introduction of the first proteasome inhibitors ${ }^{144}$. These valuable tools allowed researchers to interrogate proteasome function in complex cellular systems and tissues, and greatly advanced our understanding of many aspects of cell regulation, disease mechanisms, and immune surveillance ${ }^{66}$. Perhaps the most clinically important developments to come from the early proteasome inhibitor studies were advancements in understanding the regulation of NF-kB and its key role in the pathogenesis of many inflammatory and neoplastic diseases ${ }^{27,145}$. The first proteasome inhibitors were simple hydrophobic peptide aldehydes (analogues of serine protease inhibitors) designed to mimic the preferred substrates of the proteasome's chymotrypsin-like site (B5) and inhibit it ${ }^{27}$. The tri-peptide aldehyde, MG132 (carbobenzyl-Leu-Leu-Leu-aldehyde), is still the most widely used proteasome inhibitor in scientific research because it is potent, inexpensive, and quickly reversible ${ }^{27}$. Given the indispensable role of the proteasome in this pathway, proteasome inhibitors showed therapeutic potential for the treatment of some human 
diseases, yet it was also appreciated that complete proteasome inhibition would lead to cell death 27.

Researchers hypothesized that partial and reversible inhibition of the proteasome might be beneficial in killing neoplastic cells because they lack many of the checkpoint mechanisms that protect normal cells from apoptosis ${ }^{27,146}$. Accordingly, proteasome inhibitors were preferentially toxic to transformed and patient derived malignant cell cultures rather than their non-transformed and healthy counterparts ${ }^{146,147}$. Aldehyde proteasome inhibitors (e.g. MG-132) had limited therapeutic potential in humans due to off-target effects (e.g. inhibition of cathepsin B and calpains) and poor metabolic stability ${ }^{148}$. With MG-132 as a lead compound, a team of medicinal chemists led by Julian Adams synthesized PS-341 (a dipeptide boronic acid), a slowly reversible inhibitor of the $\beta 5$ active site (with some activity toward the $\beta 2$ active site). PS-341 proved to be a potent and selective proteasome inhibitor demonstrating therapeutic activity in preclinical models of inflammatory diseases and human cancers $146,147,149$. PS-341 entered phase I clinical trials where remarkably one patient with multiple myeloma (MM) showed a complete response to PS-341 treatment ${ }^{146}$. MM is an incurable plasma cell malignancy and at that time patients had a poor prognosis due to lack of effective treatment options, making the complete response to PS341 a dramatic clinical breakthrough ${ }^{27}$. PS-341 progressed to phase II trials for MM and chronic lymphoid leukemia. Due to the remarkable success of PS-341 in phase II trials, the FDA approved PS-341 (later renamed bortezomib and marketed it as Velcade®) (Figure 4A) as a third-line treatment for relapsed and refractory multiple myeloma (RRMM) in 2003 27. Bortezomib revolutionized the treatment of $\mathrm{MM}$ and today bortezomib is approved for use as a first line therapy for MM and mantle cell lymphoma.

Despite the clinical success in treating hematological diseases, bortezomib therapy is associated with a high rate of resistance (primary or secondary) and serious dose-limiting toxicity which require reduction or discontinuation of the drug ${ }^{150}$. Advances in proteasome inhibitor chemistry 
and a better understanding of the proteasomes unique catalytic mechanism have led to the development of second generation proteasome inhibitors with improved pharmacokinetics when compared to bortezomib ${ }^{151}$. The mechanisms of available PIs and their uses in research and clinical settings are discussed in the following sections.

\section{II.B. Chemical Classes of Proteasome Inhibitors}

There are several classes of proteasome inhibitors. Like the majority of protease inhibitors, most proteasome inhibitors are short peptides designed to fit into the substrate binding site on the catalytic subunit. The activity of proteasome inhibitors depends on the pharmacophore warhead at the C-terminus, which reacts with the active site threonine nucleophile to form a reversible or irreversible covalent adducts ${ }^{51}$. Although the proteasome has three types of catalytic sites, inhibition of all three is not required to significantly affect protein degradation ${ }^{152}$. Specific $\beta 1$ or $\beta 2$ inhibition does not have a significant effect on overall protein breakdown, however, $\beta 5$ inhibition results in significantly reduced protein breakdown ${ }^{152}$. Consequently, most proteasome inhibitors target the $\beta 5$ site, although they often have some lesser activity against $\beta 1$ and/or $\beta 2$ 152.

II.B.1. Peptide Aldehydes. Based on the well-characterized serine and cysteine protease inhibitors, peptide aldehydes (e.g. MG132) were the first synthesized proteasome inhibitors. MG132 is cell-permeable and a reversible proteasome inhibitor, which makes it a valuable

research tool. Because MG132 has slow binding and fast dissociation kinetics (Kisselev and Goldberg, 2001), the effects of MG132 proteasome inhibition on cultured cells is quickly reversed by switching to inhibitor-free media. The low cost and rapid reversibility make MG-132 the most used proteasome inhibitor for research ${ }^{27}$. However, there are several limitations to MG-132. First, MG-132 in cell culture media is rapidly oxidized into an inactive acid ${ }^{51}$. For long cell culture experiments, MG-132 containing media should be replaced daily. Second, as other peptide 
aldehydes, MG-132 also inhibits (albeit with much lower affinity) calpains and cathepsins ${ }^{51}$, therefore it is necessary to perform control experiments to confirm that the observed effects are due to proteasome inhibition. Proteasome involvement can be verified using a more selective proteasome inhibitor (e.g. epoxomicin, boronates, and lactacystin), although these compounds may be cost prohibitive for routine studies/screens. Additionally, inhibitors that specifically block other proteases (e.g. E-64 for calpains), but not the proteasome, can be used to confirm the observed effect is not due to off target inhibition of another protease.

II.B.2. Peptide Boronates. Peptide boronates are significantly more potent proteasome inhibitors when compared to peptide aldehydes. Like peptide aldehydes, peptide boronates form a tetrahedral adduct with the active site threonine, but their dissociation rate is much slower making boronates practically irreversible over hour scale time courses ${ }^{51}$. MG-232, the boronate version of MG-132, is 100 -fold more potent than its aldehyde counterpart ${ }^{51}$. Additionally, peptide boronates are not oxidized into inactive forms like MG-132, making them more stable in vivo ${ }^{51}$. The boronate pharmacophore cannot react with active site cysteines, so they have fewer nonproteasome targets ${ }^{51}$.

II.B.3. Epoxomicin \& Epoxyketones. Another naturally derived proteasome inhibitor is epoxomicin, an actinomycete fermentation metabolite. It is a modified peptide that contains a C terminal $\alpha^{\prime}, \beta^{\prime}$-epoxyketone group attached to an aliphatic P1 amino acid ${ }^{51}$. Epoxomicin is extremely specific for the proteasome. The crystal structure of yeast $20 \mathrm{~S}$ proteasome in complex with epoxomicin revealed its unusual mechanism of action and explained the basis for proteasome specificity ${ }^{57}$. Epoxomicin reacts covalently with both the catalytically active $\mathrm{N}$ terminal threonine hydroxyl and the free amino group, producing a highly stable and irreversible morpholino ring ${ }^{57}$. Unlike other PIs that only form a bond with the threonine hydroxyl, the double covalent bond formation of the epoxyketone group limits its reactivity to the $\mathrm{N}$-terminal nucleophile threonine proteases without inhibiting any other cellular protease ${ }^{64}$. Since the discovery of 
epoxomicin as a proteasome inhibitor many $\alpha^{\prime}, \beta^{\prime}$ epoxyketone electrophiles have been incorporated into peptides sequences and optimized for binding to the proteasome $\beta$ subunits . The most well-characterized epoxyketone inhibitor is carfilzomib (Kryopolis®, Onxy Pharm / Takeda) (Figure 4A), a second-generation FDA approved proteasome inhibitor for treatment of RRMM and is discussed further in Part IV.

II.B.4. Lactacystin and beta-lactone. Lactacystin, a Streptomyces metabolite, is a non-peptide proteasome inhibitor. Lactacystin itself does not inhibit the proteasome, but at neutral $\mathrm{pH}$ lactacystin spontaneously converts to clasto-lactacystin- $\beta$-lactone which is reactive with the proteasome. The $\beta$-lactone reacts with the proteasome active site threonine resulting in opening of the $\beta$-lactone ring and acylation of the proteasome catalytic threonine hydroxyl ${ }^{64}$. The yeast $20 S$ proteasome in complex with lactacystin crystal structure confirmed this mechanism providing strong evidence that an acyl enzyme conjugate is an intermediate in proteasome catalysis ${ }^{64}$. Lactacystin is more proteasome-specific than MG132, with a single off target substrate, cathepsin A. Although lactacystin is considered an irreversible proteasome inhibitor, its adduct is slowly water hydrolyzed $\left(t_{1 / 2} \sim 20 \mathrm{hr}\right)$. Lactacystin is the least stable of the proteasome inhibitors and exists in vivo in equilibrium with lactathione, its glutathione reaction product ${ }^{51}$. Despite this drawback, the high proteasome selectivity makes lactacystin a viable proteasome inhibitor for investigating the role of the proteasome in cellular processes.

II.B.5. Vinyl sulfones. Peptide vinyl sulfones are a class of irreversible proteasome inhibitors. Peptide vinyl sulfones also inhibit cysteine proteases (e.g. cathepsins), but changing the functional groups in the inhibitor's peptide portion can modulate their specificity ${ }^{153}$. For example, replacing the benzyloxycarbonyl (Z) group with the 3-nitro-4-hydroxy-5-iodophenylacetate (NIP) group in ZLVS (Z-Leu3-VS) generates NLVS significantly reducing inhibition of cathepsins B and $S^{51}$. Peptide vinyl sulfones are easy and inexpensive to synthesize, and their irreversible binding makes them attractive as protease activity probes. Peptide vinyl sulfones are commonly labeled 
with fluorophores, biotin, or a radioactive moiety, and specific uses as proteasome activity probes are discussed in Part III. Interestingly, vinyl sulfones are more potent and more trypsin-like siteselective inhibitors than epoxyketones with an identical peptide sequence ${ }^{154}$, a feature exploited in the development of $\beta 2$ specific proteasome inhibitors, as discussed in Part IV.

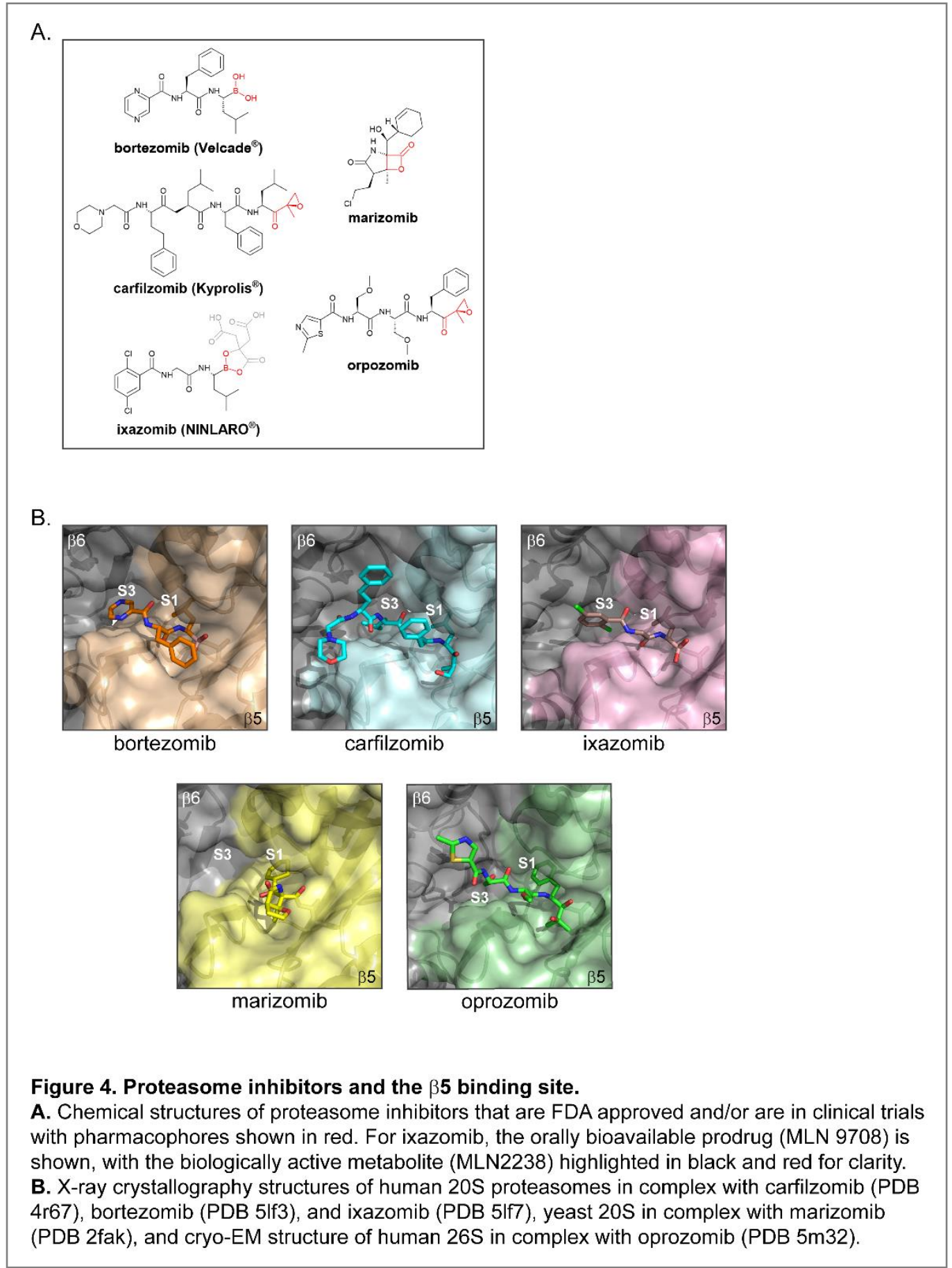




\section{II.C. Considerations for proteasome inhibitor design}

In this section we review the structural features that have been exploited to design specific inhibitors of the 20 S catalytic sites.

II.C.1. Substrate binding pockets. All six proteasome catalytic subunits (constitutive and immune) have a similar substrate binding site topology, in which the $S 1$ position is buried in the subunit next to the threonine, the S2 is solvent exposed, and both the catalytic subunit and its neighbor contribute to the S3 binding position ${ }^{155}$ (Figure 4B). However, residues that make up the S1 and S3 sites have very different catalytic subunit properties. Modification of the P1 and P3 sites on a proteasome inhibitor can significantly alter their subunit specificity and affinity. The $\beta 5 \mathrm{c}$ prefers a small hydrophobic group in P1 and a large hydrophobic group in P3, whereas $\beta 5$ i favors the inverse arrangement ${ }^{64}$. Therefore, altering the hydrophobic group size in P1 and P3 confers selectivity for $\beta 5 \mathrm{c}$ or $\beta 5 \mathrm{i}$. Due to solvent exposure in the $\mathrm{P} 2$ position, it can accommodate a range of moieties without affecting proteasome binding and is often the site for modifications aimed at improving inhibitor solubility and stability. P2 is also a useful attachment site for fluorescent probes, biotin tags, or azide handles (discussed further below).

II.C.2. Structural analysis of bound inhibitors. Knowledge of protein structure and its interaction with ligands, guides drug discovery and design. X-ray crystallography is an excellent method for obtaining high resolution proteasome structures in complex with inhibitors and has been instrumental in understanding proteasome function and advancing proteasome inhibitor development ${ }^{62}$. The early structures of ALLN and lactacystin bound proteasomes provided clues as to the threonine catalytic mechanism and intermediate states ${ }^{62}$. The structures of a substrate analog bound proteasome showed long range allosteric changes that occur upon substrate binding in the active site. The inhibitor-bound structure can be used together with biochemical data for structure-activity relationship (SAR) studies and subsequent lead compound optimization. 
There are drawbacks to using crystallography to study proteasome inhibitor - proteasome interactions. First, solvent conditions and inhibitor concentrations used in co-crystallization are not physiological and should be considered when interpreting the resultant structure. 20S proteasome crystals are usually soaked in solutions with high inhibitor concentrations under conditions that preserve crystal integrity, whereas enzymatic assays are carried out at $37^{\circ} \mathrm{C}$ with low inhibitor concentrations and proteasomes under conditions optimized for substrate degradation. Discordant data in the yeast $20 \mathrm{~S}$ proteasome structure in complex with Ac-LLN-al showed the inhibitor bound to all six proteasome active sites, but the biochemical data indicated that Ac-LLN-al proteasome inhibitor preferentially inhibits the $\beta 5$ site (with very low activity against the $\beta 1$ and $\beta 2$ ) unless used at extremely high concentrations ${ }^{64}$. If one considers that most proteasome inhibitors affects multiple active sites at high concentrations and that proteasome inhibitor concentration in the crystallization condition was in the millimolar range, it is unsurprising that the inhibitor bound all sites. This example illustrates the necessity to carefully consider existing biochemical data when interpreting new structures.

Additionally, obtaining good diffraction data relies on homogenous crystal packing. Under these conditions, one may miss larger scale conformational changes that take place upon $20 \mathrm{~S}$ proteasome ligand binding. The inherent drawbacks in crystallography methodology highlight the need to incorporate other structural methods into understanding the proteasome. Recent advances in cryo-electron microscopy (EM) and single particle analysis make it possible to obtain near atomic level resolution protein structures in more physiological conditions ${ }^{156}$. Proof of principle for the cryo-EM utility in structure-based drug discovery and development is found in ${ }^{157}$. Recently, the noncovalent reversible asparagine-ethylenediamine (AsnEDA)-based inhibitor bound human immuno-proteasome cryo-EM structure was used in SAR studies ${ }^{157}$. Exploiting $\beta 5 c / \beta 5 i$ residue differences near the S1 pocket improved PKS21187 affinity for $\beta 5 i$ down to 15 $\mathrm{nM}$ (from $58 \mathrm{nM}$ ) and successfully improved selectivity (20 fold) over $\beta 5 \mathrm{c}{ }^{156}$. Although cryo-EM 
typically cannot provide quite the same resolution as crystallography, the $20 \mathrm{~S}$ core particle characteristically has high local resolution ( $\sim 3 \AA$, in both above studies) at the interior of the $\beta$ subunits, making this a valuable method for investigating proteasome ligand binding under relatively physiological conditions.

It is important to keep in mind that cryo-EM structures are derived from averaging classes of particles, meaning that protein sub-conformations may be overlooked. This is important in interpreting $26 \mathrm{~S}$ proteasome structures because the complex undergoes large-scale conformational changes during cycles of substrate binding and ATP hydrolysis, resulting in many conformational states coexisting simultaneously. Despite physiologically relevant conditions, conformer subpopulations may not be apparent. For example, Baumeister and colleagues published $26 \mathrm{~S}$ proteasome in the ATP-hydrolyzing state ${ }^{74}$ and the ATP-yS bound cryo-EM structures ${ }^{72}$. However, when they performed a deep classification of more than 3 million $26 \mathrm{~S}$ proteasome particles in the presence of both ATP and ATP-yS they identified a third state of the 265 proteasome that was believed to be an intermediate conformation during the ATP hydrolysis cycle ${ }^{158}$. Additional intermediate $26 \mathrm{~S}$ conformations states have been identified in humans and yeast using cryo-EM ${ }^{159-161 .}$

II.C.3. Proteasome inhibitor pharmacophore properties. As previously discussed, pharmacophores confer specific proteasome inhibitor properties including compound stability, off target protease inhibition, and inhibition kinetics. Interestingly, the pharmacophore nature is suggested to influence proteasome inhibitor active site specificity. Epoxyketone warhead replacement with vinyl sulfone moieties in $\beta 5$ inhibitors further improves $\beta 5$ site (but not $\beta 5 i$ site) selectivity ${ }^{153}$. Therefore, each warhead confers unique properties to the proteasome inhibitor and thus careful considerations needs to be made when selecting a pharmacophore along with appropriate controls. 


\section{Methods for pharmacological proteasome research}

Extensive methodology exists for investigating proteasome function in vitro and in vivo. Here, we describe some commonly used methods for proteasome purification, peptidase activity assays, and protein degradation assays in vitro and in cell culture, and discuss their advantages and limitations.

\section{III.A. Proteasome purifications}

Rigorous and reproducible studies of proteasome pharmacology require a source of pure and active proteasomes. The following is a summary of methods for endogenous and affinity tagged proteasome purifications.

III.A.1 Endogenous proteasome purification. Endogenous proteasomes are purified from a variety of tissues using a series of anion exchange chromatography columns. After purification, $20 \mathrm{~S}$ and $26 \mathrm{~S}$ proteasomes are separated by gel filtration or glycerol gradient centrifugation. Because 26S proteasomes require ATP hydrolysis to remain intact, omitting ATP from the homogenization and chromatography buffers enriches for $20 \mathrm{~S}$ proteasomes. Rabbit skeletal muscle and bovine liver have abundant proteasomes making it possible to obtain pure proteasomes (>95\%) in milligram quantities in under a week. Most 20 S proteasome preparations still use this method today.

Another method to purify endogenous $26 \mathrm{~S}$ proteasomes from almost any tissue or cell type takes advantage of the 195 regulatory particle's affinity for proteins containing ubiquitin-like (UBL) domains ${ }^{162}$. Recombinant glutathione S-transferase (GST) fused to the ubiquitin-like (UBL) domain of ubiquitin shuttling factor RAD23B is purified from $E$. coli and bound to glutathione beads. 26 S proteasomes in cell or tissue lysates bind the GST-UBL column while all other cellular proteins are washed away. Bound $26 \mathrm{~S}$ proteasomes are subsequently eluted by adding high 
concentrations of a tandem ubiquitin-interacting motif (UIM) derived from the 19S ubiquitin binding subunit, Rpn10 162162. Unlike anion exchange chromatography (which takes $\sim 3$ days), the UBLaffinity method is completed in a single day without high salt buffers. Rapid and gentle 26S purification is essential to retain loosely associated proteasome proteins which are lost during anion exchange chromatography.

Because the GST-UBL bait occupies UBL binding sites on the 19S, endogenous UBL domain containing proteins and Ub-conjugates may be dislodged from the proteasome upon purification 163. Despite this limitation, the UBL-affinity method has been valuable in studies investigating $26 \mathrm{~S}$ proteasome composition in a variety of physiological and disease states. For example, Qiu et al., (2006) used the UBL-affinity method to identify Rpn13 ${ }^{164}$, a novel human $19 S$ subunit that tethers and activates UCHL5 (a deubiquitinase) to the 26S proteasome and functions as a ubiquitin receptor ${ }^{165}$. The UBL-affinity method also co-purifies other important proteasome associated proteins with important roles in regulating proteasome function and Ub-conjugate degradation (e.g. the deubiquitinase USP14) ${ }^{163}$. A significant advantage of the UBL-affinity method is that you can purify proteasomes from diseased tissues and study the changes in proteasome activity and composition without genetic alterations.

Most commercially available 20S and 26S proteasomes are purified from mammalian (e.g. human, rabbit) erythrocytes. Since erythrocytes lack nuclei, these endogenous proteasomes are "aged", perhaps with oxidative damage, and may have lower basal activity than those derived from nucleated cells.

III.A.2 Affinity-tagged proteasomes. Several groups have created human cell lines and yeast strains stably expressing affinity tagged proteasome subunits to rapidly isolate proteasomes for structural and functional studies. Affinity tags are often appended to the C-termini of Rpn11 or $\beta 4$ 
because modifications on these subunits do not cause any discernable effect on proteasome function in cellular or yeast cultures.

Affinity-tagged proteasome purifications are especially useful for studies of changes in $26 \mathrm{~S}$ proteasome composition because they typically co-purify with more Ub-conjugates and additional proteasome-interacting proteins than UBL-affinity or ion exchange chromatography methods. For example, Leggett et al., (2002) used a TEV-protease cleavable Protein A derived tags on Rpn11, Rpt1, and $\beta 4$ to study how proteasome interacting proteins (PIPs) regulate the stability of the yeast $26 \mathrm{~S}$ complex in vivo ${ }^{167}$. The high purity and yield of affinity-tagged proteasomes is well suited for cryo-EM studies. For example, Matyskiela et al., (2013) used cryo-EM analysis of Rpn11-3xFLAG yeast proteasomes to study the conformational dynamics during 26S substrate engagement ${ }^{75}$. Affinity tags are also amenable to high purification efficiency of crosslinked complexes under fully denaturing conditions. Guerrero et al., (2006) designed a tandem affinity tag consisting of a hexahistidine sequence and an in vivo biotinylation signal (HB) ${ }^{168}$. Tandem affinity purification of Rpn11-HB proteasomes after in vivo cross-linking was combined with tandem mass spectrometry (MS) and quantitative SILAC (stable isotope labeling of amino acids in cell culture) to globally map the $26 \mathrm{~S}$ proteasome interaction network in yeast ${ }^{168}$. A TEVcleavable version of the HB tag (HTBH and HBTH) allows for one-step purification of human $26 \mathrm{~S}$ proteasomes. Wang et al., (2017) generated several stable HEK293 cell lines expressing tagged subunits (e.g. Rpn11-HTBH, HBTH-Rpn1, HBTH-Rpt6) ${ }^{170 . ~ T h e y ~ u s e d ~ t h e s e ~ c e l l s ~ l i n e s ~ w i t h ~ i n ~}$ vivo and in vitro crosslinking-MS workflows and cryo-EM approaches to comprehensively examine protein-protein interactions within the $26 S$ proteasome ${ }^{169}$. Choi et al. (2016) developed stable $\beta 4-H T B H / \alpha 3-F L A G$ and $\beta 4-H T B H / \alpha 3 \Delta N-F L A G$ HEK293 cell lines ${ }^{171}$. The $\alpha 3 \mathrm{~N}$-terminal deletion $(\alpha 3 \Delta N)$ results in a constitutively open gate. These cell lines are an excellent tool for investigating the role of dynamic proteasome gating, and are discussed further in Part V. It is worth noting that most of these cell lines stably express the tagged subunit in addition to the 
endogenous gene, thus purification does not isolate all proteasome complexes and may not reflect all changes in proteasome composition under different physiological states or drug treatments.

III.A.3. Immunoproteasomes. Immunoproteasome preparations are usually purified from spleens and cell cultures treated with IFN- $y$ to increase immunoproteasome subunit expression. Immunoproteasomes can be purified using the same anion exchange chromatography methods as described above for constitutive proteasomes. However, constitutive proteasomes are ubiquitously present in all tissue types and even low amounts can interfere with immunoproteasome-specific research. Dechavanne et al., (2013) report that hydrophobic interaction chromatography can successfully separate immunoproteasomes from residual constitutive proteasome contamination after purification ${ }^{172}$.

III.A.4. Validating and storing proteasome preparations. It is imperative to check for contaminating proteases in all proteasome preparations. For example, the anion exchange elution profile of tripeptidyl-proteases (TPP) overlaps with proteasomes. TPP is a serine protease and capable of cleaving fluorogenic peptides (e.g. Suc-LLVY-amc, Ac-AFF-amc). Proteasome specific activity can be verified with proteasome inhibitors as a negative control.

Regardless of the purification method, it is necessary to confirm the $20 \mathrm{~S} / 26 \mathrm{~S}$ proteasome assemblies in your preparation. For example, purifying affinity tagged RPN11 proteasomes will select for single capped 20S, double capped 20S, and free 19S particles, while affinity tagged 20 S subunits selects for free 20S, 26S, and 20 S associated with other regulators (e.g. PA28, PA200). 20S proteasomes and 20 S bound to regulators are clearly separated by native-PAGE (e.g. NuPAGE® 3-8\% tris-acetate gel) ${ }^{162}$. Electrophoresis with $26 \mathrm{~S}$ proteasomes are performed at $4^{\circ} \mathrm{C}$ with adequate ATP and $\mathrm{MgCl}_{2}$ in the buffer to prevent complex dissociation (Table 1). After electrophoresis peptidase activity of proteasome complexes is measured by an in-gel 
fluorescence activity assay ${ }^{162}$. The gel is incubated at $37^{\circ} \mathrm{C}$ in buffer containing the fluorogenic peptide substrate Suc-LLVY-amc and the cleaved amc fluorophore is visualized with ultraviolet light. Adding $0.02 \%$ sodium dodecyl sulfate the gel incubation buffer enhances 20 S peptidase activity and improves visualization of the $20 \mathrm{~S}$ band. After UV imaging, the gel can be processed with coomassie/silver stain, analyzed by 2D native-SDS-PAGE, or transferred to a membrane for immunoblot analysis. Due to the large size of the proteasome complexes (700kDa-2.4MDa), incubating the gel in SDS buffer prior to transfer may improve transfer efficiency and increase epitope availability. Roelofs et al., (2018) provide methods for various downstream analyses to investigate the activity and composition of proteasome complexes separated by native-PAGE ${ }^{173}$.

The association between the $19 \mathrm{~S}$ and $20 \mathrm{~S}$ is labile and sensitive to changes in temperature and presence of nucleotides. To support integrity of $26 \mathrm{~S}$ proteasome complexes, purification should be performed as rapidly as possible in the presence of adequate ATP and $\mathrm{MgCl}_{2}$ and always at $4^{\circ} \mathrm{C}$. Addition of glycerol ( 10\%) in the proteasome purification and storage buffers stabilizes the $26 \mathrm{~S}$ complexes and the latent 20S gate. After purification, both $20 \mathrm{~S}$ and $26 \mathrm{~S}$ proteasomes should be flash frozen in liquid nitrogen, stored at $-80^{\circ} \mathrm{C}$, and never refrozen once thawed. It is important to thaw frozen 265 proteasomes on ice and use them immediately after thawing. Since freezing and storage conditions can affect the labile $26 \mathrm{~S}$ proteasome assembly, we recommend verifying the assembly state of your 26S preparation after thawing with native-PAGE.

\section{III.B. Monitoring proteasome activity}

There are numerous methods for monitoring proteasome activity in vitro and in vivo. In vitro experiments are performed with either peptide-based or protein-based model substrates. The following section covers commonly used peptide and protein-based model proteasome substrates and methods for monitoring their degradation. Lastly, we will discuss artificial proteasome substrates for expression in cell culture and transgenic animals. 


\section{TABLE 1 | EXPERIMENTAL PITFALLS}

Buffers

- ATP \& Magnesium: Stability of the $26 \mathrm{~S}$ complex is dependent on a high ATP:ADP ratio. For monitoring 265 activity, an adequate amount of ATP and $\mathrm{MgCl}_{2}$ in a $1: 5$ ratio (we typically use $2 \mathrm{mM}$ and $10 \mathrm{mM}$, respectively) should be present in all buffers to maintain the stability of the $26 \mathrm{~S}$ proteasome complex. At the same time, the levels of ADP should be kept at a minimum.

- Glycerol: Glycerol in the buffer stabilizes and maintains the closed, latent gate. Typically, purification buffers for proteasomes contain $10 \%$ glycerol, and assay buffers contain $5 \%$.

- Sodium dodecyl sulfate (SDS): Addition of $0.02 \%$ SDS to assay buffer mildly stimulates opening of the latent $20 \mathrm{~S}$ gate.

Proteasome populations

- Proteasomes are present as individual 20S particles and 26S particles (singly caped 20S-19S and doubly capped 19S-20S-19S). The 26S complex can dissociate into the $19 S$ and $20 S$ constituents over time, after a freeze/thaw, or in response to buffer condition changes.

- When performing experiments with purified $26 \mathrm{~S}$ proteasomes, it is important to determine the ratio of intact $26 \mathrm{~S}$ and free $20 \mathrm{~S}$ proteasomes. This is commonly accomplished by native-PAGE electrophoresis as described in the text.

\section{Microplates}

- The type of microplate (e.g. untreated vs non-binding surface treatments, polypropylene vs polystyrene) can affects the observed activity, Some substrates (e.g. GFP) interact with and "stick" to untreated plate surfaces. Some compounds or small substrates may bind to the plate surface and reduce the effective concentration in the assay. It is worthwhile to confirm results with new compounds/substrates by using two or more types of plates. BSA can be included in the buffer to prevent nonspecific binding to the plate.

Proteasome gating studies

- Experiments probing the role/regulation of the proteasome gate might be facilitated by using the "open-gate" $20 S$ mutant, $\alpha 3 \Delta \mathrm{N}$.

- Suc-LLVY-amc has been shown to mildly stimulate gate opening, so another substrate (e.g. Ac-nLPnLD-amc) should be used in conjunction.

III.B.1. Peptide-based model substrates: In vitro proteasome activity is often measured using a fluorescent substrate enzyme activity assay. Peptide substrates are useful for monitoring proteasome gating and peptidase activities and are amenable to high throughput formats. Model peptide substrates are short tri- or tetrapeptides with a C terminal fluorophore (e.g. 7-amino-4methylcoumarin, amc). The amino acid sequences are designed to preferentially interact with and be degraded by specific $20 \mathrm{~S}$ subunits (Table 2, Figure 2B). Common peptide substrates are SucLLVY-amc (Succinyl-leucine-leucine-valine-tyrosine-amc), Boc-LRR-amc (Tert-butoxycarbonyl-

\section{TABLE 2 | FLUOROGENIC PEPTIDES}

\begin{tabular}{|c|c|c|}
\hline Subunit & Preference & Substrate \\
\hline$\beta 1$ & acidic & $\begin{array}{c}\text { Ac-nLPnLD-amc } \\
\text { Z-LLE-amc }\end{array}$ \\
\hline$\beta 1 i$ & hydrophobic & Ac-PAL-amc* \\
\hline$\beta 2$ & basic & $\begin{array}{l}\text { Boc-LRR-amc } \\
\text { Ac-RLR-amc }\end{array}$ \\
\hline$\beta 2 i$ & basic & $\mathrm{n} / \mathrm{a}$ \\
\hline$\beta 5$ & hydrophobic & $\begin{array}{c}\text { Suc-LLVY-amc } \\
\text { Ac-WLA-amc }\end{array}$ \\
\hline$\beta 5 i$ & hydrophobic & Ac-ANW-amc* \\
\hline
\end{tabular}

* these substrates differentiate between $\beta 5$ and $\beta 5$ i activity.

$n / a$, substrates specific for $\beta 2 i$ have not been developed to date. leucine-arginine-arginine-amc), and Ac-nLPnLD-

aminoluciferin ( $\mathrm{N}$ acetyl-norleucinal-proline-norleucinalaspartate-amc) for the $\beta 5$ chymotrypsin-like, $\beta 2$ trypsinlike and $\beta 1$ caspase-like activities, respectively. However, it is important to note that at high substrate concentrations multiple active sites can participate in substrate cleavage. The amc moiety is quenched at the excitation wavelength (ex: $380 \mathrm{~nm}$ ) when attached to the peptide, and proteolytic 
cleavage enhances fluorescence (em: $460 \mathrm{~nm})$. Changes in fluorescence intensity are monitored in real time using a microplate reader. The increases in fluorescence intensity is directly proportional to proteasome proteolytic activity. This assay is rapid and suitable for high-throughput studies. Cell-based reagent kits use similar aminoluciferin-fused peptide substrates which allows measurement of proteasome peptidase activity in intact cells.

Although fluorescent substrate peptides are an excellent tool for preliminary studies measuring proteasome activity (e.g. screening compound libraries), there are several pitfalls. Foremost, small peptides bypass the need for 195 recognition and unfolding and thus report on only $20 \mathrm{~S}$ peptidase activities. Furthermore, changes in peptide hydrolysis does not necessarily correlate with changes in protein degradation, and it is important to note that at high peptide substrate concentrations multiple active site types can participate in peptide cleavage. As such, chymotrypsin-like ( $\beta 5$ ) activity (Suc-LLVY-amc) assays evaluating response to a proteasome inhibitor may overestimate the reduction in protein degradation in vivo. Therefore, it is imperative to evaluate the experimental effects with full length protein degradation and not rely solely on peptide hydrolysis data.

III.B.2. Protein based model substrates. Many protein substrates are available for monitoring $20 S$ and 265 proteasome degradation in vitro (e.g. using purified proteasomes or cellular lysates). Here we describe methods for quantitating protein degradation and give examples of model substrates for $20 \mathrm{~S}$ and $26 \mathrm{~S}$ proteasome activity.

Methods to quantitate protein degradation. The extent of in vitro protein degradation can be measured in several ways. The fluorescamine assay is a quantitative method for measuring protein cleavage products generated by the proteasome. The proteasome processively degrades proteins and the products generated equals the number of substrate molecules degraded multiplied by the mean number of cuts made in a single polypeptide ${ }^{152}$. Fluorescamine addition 
to an amine free assay buffer quickly labels new $\mathrm{N}$-termini amines on short peptides generated by proteolytic cleavage.

Alternatively, the proteins can be separated by SDS-PAGE and monitored for substrate band disappearance via coomassie staining, silver staining, or immunoblot. Care must be taken when monitoring degradation via western blot, as degradation of the epitope results in complete loss of signal, which may not corollate with complete protein degradation. Since most gel-based protein degradation assays are performed with small reaction volumes $(<20 \mu \mathrm{L})$ incubated in centrifuge tubes, the amount of substrate remaining should be normalized to a loading control (e.g. a 20S proteasome subunit) to control for loss of substrate protein during pipetting or incubation steps. Fluorescence anisotropy is useful to follow the degradation of fluorescent dye labeled protein substrates of $20 \mathrm{~S}$ and $26 \mathrm{~S}$ proteasomes in real time ${ }^{174,175}$. Bhattacharyya et al. (2018) describe methods for high throughput measurement of $26 \mathrm{~S}$ ubiquitin-dependent degradation using dye labeled substrates ${ }^{174}$.

$20 S$ substrates (19S independent). For example, $\beta$-casein is a good substrate for monitoring $20 \mathrm{~S}$ protein degradation since it is unstructured, thus abrogating the requirement for the unfoldase activity associated with the $19 \mathrm{~S}$ regulatory particle and is commercially available.

265 (ubiquitin independent) substrates. Folded substrates are required to determine the contribution of 195 activities toward 26S proteasome degradation. Ornithine decarboxylase (ODC) is a stably folded protein containing a C-terminus degradation tag ${ }^{176}$ that promotes rapid Ub-independent degradation by $26 \mathrm{~S}$ proteasomes ${ }^{177}$. Fusing the ODC degradation tag (CODC) to other proteins promotes their proteasomal degradation ${ }^{178}$. For example, cODC fusion to the titin I27 domain allows for testing of the ubiquitin-independent degradation of a folded protein, though the kinetics may be slow ${ }^{179}$. Destabilizing mutations can be introduced to 127 and accelerate substrate degradation ${ }^{179}$. 
265 (ubiquitin dependent) substrates. The 265 ubiquitin-dependent degradation of folded proteins can be monitored with a tetra-ubiquitin fused green fluorescent protein (GFP) ${ }^{180}$ with a C-terminal unstructured region ${ }^{181}$ or a tetra-ubiquitinated dihydrofolate reductase (DHFR) ${ }^{182}$. It is also possible to express some cODC fusion proteins in vivo to monitor proteasome activity.

III.B.3. Proteasome activity in cell culture. The $26 \mathrm{~S}$ proteasome degrades ubiquitinated proteins and proteasome impairment leads to poly-ubiquitinated protein accumulation. Measuring changes in high molecular weight poly-ubiquitin protein conjugates via immunodetection is a method to monitor changes in proteasome activity. This is a general, non-specific method to measure changes in proteasome degradation and should not be used alone to assess proteasome activity.

Stable expression of GFP-fusion reporters is commonly used in cell culture to monitor proteasome activity. Measuring fluorescent reporters is a well-established technique for monitoring proteasome activity. Changes in reporter protein levels (in the absence of translation changes) inversely reflects degradative capacity of the UPS. Wild type GFP has a long half-life in mammalian cells and therefore is not a suitable proteasome degradation substrate for most experiments. Several GFP-fusion proteins have been engineered as specific proteasome substrates (Table 3). Bence et al. (2005) designed a synthetic reporter consisting of the short degron, CL1 ${ }^{183}$, a consensus sequence for ubiquitination that was first identified in fission yeast 184, fused to the C-terminus of GFP (GFPu), thereby targeting it for ubiquitin-dependent proteasome degradation ${ }^{183}$. Addition of the CL1 degron converted the GFP half-life from $\sim 10$ hours to $\sim 30$ minutes. Cell compartment-specific proteasome function can be monitored by localization of GFPu directed to the nucleus (NLS- GFPu), the cytoplasm (NES-GFPu) ${ }^{183,185}$, or to neuronal synapses (PSD95- GFPu and SNAP25- GFPu) ${ }^{186}$ (Table 3). 
Other UPS reporters have been generated to determine targeted proteasome degradation using different pathways. The $\mathrm{N}$-end rule relates the cellular protein half-life to the identity of its $\mathrm{N}$ terminal residue ${ }^{187}$. Fluorescent substrates of the $\mathrm{N}$-end rule degradation pathway have been created with ubiquitin-GFP fusion constructs. When these ubiquitin fusion proteins are expressed in cells, deubiquitinases rapidly cleave the ubiquitin and expose an unmodified N terminal GFP residue. $\mathrm{N}$ terminal arginine residue exposure (e.g. the substrate Ub-R-GFP) recruits UBR E3 ligases which ubiquitinate the protein, targeting it for proteasome degradation ${ }^{188}$. Techniques for generating ubiquitin fusion proteins with varying half-lives and conditional mutants are described in ${ }^{189,190}$. Unlike Ub-R-GFP, the reporter Ub ${ }^{\mathrm{G} 76 V}$-GFP cannot be deubiquitinated (thereby bypassing the $\mathrm{N}$-end rule pathway) and is a model substrate for the in vivo Ub fusion degradation (UFD) pathway ${ }^{188}$. The $T$ cell receptor protein $\alpha$ chain is rapidly degraded in nonhematopoietic cells, and a TCRa-GFP fusion protein can monitor ERAD specific proteasome activity ${ }^{191}$. Protein synthesis also influences the steady state protein levels, and synthesis rates can be affected by cellular stress, transfection efficiency, and varies from cell to cell. Therefore, it is imperative to utilize appropriate experimental design to take expression and translation differences into account when monitoring protein degradation. Commonly used methods are pulse-chase experiments, cycloheximide chase experiments, bicistronic constructs, or the parallel measurement of stable, long lived fluorescent reporters. Lastly, each substrate only illustrates a single degradation pathway, which may or may not accurately reflect the UPS perturbations within a cell. It is advantageous to consider the use of multiple such proteasome substrates to confirm findings.

In addition to cellular proteasomal activity, proteasome localization and dynamics can also be monitored with fluorescently labeled proteasome subunits. Both $\alpha$ and $\beta$ subunits can be fused to a fluorescent protein and have been shown to efficiently incorporate into proteasome particles. For example, a4-YFP ${ }^{192}$ and a CFP-tagged $\beta 1 i^{193}$ have been used to monitor localization 
dynamics of constitutive and immunoproteasomes in living cells. Detailed methods for monitoring proteasome dynamics in living cells are described elsewhere ${ }^{194}$.

\begin{tabular}{|c|c|c|c|c|}
\hline Substrate & Ub / pathway & Localization & $\mathrm{t} 1 / 2$ & Ref \\
\hline GFPu & Yes / CL1 degron & & $30 \mathrm{~m}$ & Bence et al., 2001 \\
\hline NLS-GFPu & Yes / CL1 degron & Nuclear & $60 \mathrm{~m}$ & Bennett et al., 2005 \\
\hline NES-GFPu & Yes / CL1 degron & Cytosolic & $60 \mathrm{~m}$ & Bennett et al., 2005 \\
\hline PSD95-GFPu & Yes / CL1 degron & Postsynaptic & ND & Wang et al., 2008 \\
\hline SNAP25-GFPu & Yes / CL1 degron & Presynaptic & ND & Wang et al., 2008 \\
\hline TCR-a-GFP & Yes / ERAD & & ND & DeLaBarre et al., 2006 \\
\hline Ub-M-GFP & Yes / "normal" & & "Stable" & Dantuma et al., 2000 \\
\hline Ub-R-GFP & Yes / N-end rule & & "short" & Dantuma et al., 2000 \\
\hline UbG76V_GFP & Yes / UFD & & "short" & Dantuma et al., 2000 \\
\hline Ub ${ }^{G 76 V}$-Dendra2 & Yes / UFD & & ND & Hamer et al., 2010 \\
\hline GFP-ODC & No & & $2 \mathrm{~h}$ & Li et al., 1998 \\
\hline \multicolumn{5}{|c|}{$\begin{array}{l}\text { Abbreviations: GFP, green fluorescent protein; Ub, ubiquitin; } \mathrm{t} 1 / 2 \text {, in vivo half life; } N L S \text {, nuclear localization signal; } N E S \text {, nuclear export } \\
\text { signal; PSD95, post synaptic density } 95 ; \text { SNAP25, synaptosomal associated protein } 25 ; T C R-\alpha, T \text { cell receptor a chain; ERAD, } \\
\text { endoplasmic-reticulum-association-degradation; } M \text {, methionine; R, arginine; ND, not determined; ODC, ornithine decarboxylase }\end{array}$} \\
\hline
\end{tabular}

III.B.4. In vivo proteasome activity. Transgenic animals that carry UFD proteasome substrates have also been generated and are used to study proteasome function in live tissues ${ }^{195,196 .}$ Detailed methods for monitoring UFD protein degradation in yeast, cell lines, and transgenic mice are described in Menendez-Benito et al. (2005) ${ }^{197}$. The photoactivatable UbG76V-dendra2 construct monitors proteasome activity independent of translation and has been successfully used in transgenic $C$. elegans to determine tissue specific proteasome degradation rates ${ }^{198}$.

\section{III.C. Proteasome active site probes}

Activity based probes $(\mathrm{ABP})$ recognize catalytic sites on the constitutive or immuno-proteasomes without requiring genetic techniques. Most proteasome ABP probes are modified proteasome 
inhibitors with a fluorescent molecule incorporated at or near the N-terminus. ABPs have been developed that can distinguish specific constitutive and immunoproteasome subunits ${ }^{199}$. After proteasome labeling and SDS-PAGE protein separation, the modified proteasome subunits are immediately visualized via in-gel fluorescence or immunoblotting. Furthermore, cell permeable ABPs are compatible with live-cell imaging to detect real-time proteasome localization or flow cytometry-based experiments. Site selective ABPs are useful in determining novel proteasome inhibitor subunit specificity. A recent review provides a detailed account of currently available APBs $^{196}$.

\section{Proteasome inhibitors to treat human disease}

\section{IV.A. Hematological Malignancies}

IV.A.1. Bortezomib \& Multiple Myeloma. At therapeutic doses, bortezomib inhibits approximately $30 \%$ of proteasome mediated protein degradation ${ }^{146}$, which is sufficient to induce MM tumor cells apoptosis without causing general toxicity in non-transformed cells. Considering the indispensable role of proteasome function in all cell types this raises an important question: why is bortezomib particularly toxic to MM cells? First, proteasome inhibition stabilizes the NF-KB complex in the cytoplasm and reduces NF-KB dependent gene expression. MM cells have increased NF-KB activity and rely on this pathway for survival and proliferation ${ }^{200}$. Furthermore, NF-kB activity generates more pro-inflammatory NF-KB activators in a positive feedback loop, therefore partial proteasome inhibition is sufficient to reduce this pathological cascade ${ }^{201}$. Bortezomib-mediated NF-KB inhibition enhances the anti-MM conventional chemotherapeutic agents (e.g. dexamethasone, lenalidomide) effects and increases MM patient progression-free survival (PFS) and overall survival (OS) ${ }^{151}$. Second, proteasome inhibition reduces misfolded protein clearance. MM arises from mature immunoglobulin (lg)-secreting plasma cell hyper proliferation in the bone marrow and MM cells have a high rate of Ig production. Immunoglobulins are large multisubunit molecules synthesized and folded in the ER where they are post- 
translationally modified prior to secretion. The high lg production rate and multiple modifications make MM cells heavily reliant on the proteasome and ERAD to maintain ER homeostasis ${ }^{202}$ - so it reasons they will be more sensitive to proteasome inhibition. Accordingly, treatment of MM cells with proteasome inhibitors results in a toxic misfolded protein buildup activating JNK and eventually resulting in apoptosis. Third, proteasome inhibition stabilizes various tumor suppressor proteins (e.g. p27, p53) and prevent cell cycle progression ${ }^{203}$.

IV.A.2. Bortezomib Resistance. Although bortezomib revolutionized the treatment for MM, bortezomib resistance and relapse often occurs in patients who initially respond to bortezomib. Therefore, bortezomib resistance is a major issue for MM therapy. There are several mechanisms linked to bortezomib resistance and they fall into two broad categories. First, there are changes in proteasome subunit composition and expression ${ }^{204}$. Additionally, mutations in the $\beta 5$ bortezomib binding pocket are associated with bortezomib resistance in MM cell lines ${ }^{205}$. However, the same mutations have not been confirmed in MM patients resistant to bortezomib treatment. Bortezomib-resistant $\mathrm{MM}$ cells also display transcriptome alterations including increased anti-apoptotic protein and decreased pro-apoptotic protein expression ${ }^{206}$. For example, bortezomib-resistant MM cells have higher Bcl-2 family ${ }^{207}$, and heat shock protein (Hsp27, Hsp70, and Hsp90) expression, which are expected to mitigate the misfolded protein burden in these cells.

Other extrinsic factors contribute to bortezomib-resistance. Not surprisingly, the bone marrow microenvironment plays an important role in supporting bortezomib-resistance. Bone marrow stem cells (BMSC) isolated from bortezomib-resistance MM patients have different cytokine profiles than BMSCs from bortezomib-sensitive MM patients ${ }^{208}$. Several specific pro-survival cytokines and microRNAs are upregulated and contribute to bortezomib-resistance ${ }^{209}$. Interestingly, bortezomib-resistant patient BMSCs confer resistance to proteasome inhibitor naïve $\mathrm{MM}$ cells, whereas proteasome inhibitor -naïve MM cells co-cultured with bortezomib- 
sensitive MM patient BSMCs respond to subsequent bortezomib exposure ${ }^{209}$. Elucidating specific microenvironment mechanisms supporting bortezomib-resistance is expected to identify additional targets for combination therapies to enhance proteasome inhibitor sensitivity in RRMM patient.

IV.A.3. Bortezomib Dose Limiting Toxicity. The primary dose limiting bortezomib treatment toxicity is peripheral neuropathy (PN). Bortezomib induced PN typically affects the peripheral nervous system sensory fibers and is associated with a painful burning sensation, numbness, and/or tingling in the extremities. Clinical trials report bortezomib-induced PN incidence (all grades) between $30-60 \%{ }^{210}$ and grade $\geq 3$ occurring between $2-23 \%{ }^{211}$. PN is a major cause of dose reduction or discontinuation in patients and overcoming this limitation is a significant challenge to clinicians and pharmaceutical development. Recent clinical trials demonstrated that patients receiving alternative dosing schedules (i.e. once weekly, instead of bi-weekly) or subcutaneous injection of bortezomib (instead of IV) had a lower incidence of PN, without changes in efficacy. For patients with intolerable PN, these options constitute another avenue of hope before discontinuing treatment ${ }^{211}$.

Although the exact molecular mechanisms by which bortezomib induces PN are not completely clear, clinical and experimental evidence points to pathology in the primary sensory neurons cell bodies as a major contributing factor. The peripheral nervous system (PNS) encompasses the nerve fibers and cell bodies that reside outside the central nervous system (CNS, i.e. brain and spinal cord). Sensory receptors in periphery tissues transduce physical stimuli (e.g. pain, touch, pressure, temperature) into action potentials which are transmitted via primary sensory neurons to the CNS. The primary sensory neuron cell bodies are in the dorsal root ganglion (DRG) just outside of the spinal cord. Many in vivo mouse studies and in vitro DRG explant studies of bortezomib induced PN demonstrate accumulation of ubiquitinated proteins in DRG soma, defects in mitochondrial calcium homeostasis, disrupted mitochondrial axonal transport, 
alterations in tubulin polymerization and localization, and defects in fast axonal transport due to blockage of axonal protein turnover ${ }^{212-214}$.

Why are the DRG neurons especially susceptible to proteasome inhibitor toxicity? Proper CNS and PNS neural function requires an exquisitely controlled microenvironment. To this end, the blood-neural barrier (BNB) and the blood-brain barrier (BBB) form a protective barrier between the changing circulatory milieu and the PNS and CNS, respectively. These intricate barriers contain complex tight junction proteins. Unlike the rest of the nervous system, the cell body-rich area within the DRG has endothelial fenestrations and lacks tight junction proteins, rendering it more permeable to substances in the blood compared to the rest of the nervous system. This region is highly vascularized and blood permeability has been observed in human subjects using magnetic resonance imaging (MRI) with gadolinium contrast agents ${ }^{210}$. While bortezomib cannot cross the tight BNB and BBB, it can cross into the cell body-rich region of the DRG and inhibit proteasome function. This differential permeability is thought to underlie peripheral sensory system vulnerability to cytostatic agents used in chemotherapy (e.g. bortezomib) compared with other neurons in the CNS ${ }^{210}$.

Arastu-Kapur et al. (2011) suggest bortezomib induced neurotoxicity is due to mitochondrial serine protease, HtrA2/Omi, inhibition and independent of proteasome inhibition ${ }^{215}$. They also showed that a second-generation proteasome inhibitor, carfilzomib, did not inhibit HtrA2/Omi in this study. The authors conclude that proteasome inhibitor induced neurotoxicity is due to offtarget bortezomib effects, and not generalizable to the proteasome inhibitor class. However, two subsequent independent studies failed to show bortezomib-mediated HtrA2 inhibition 216,217. Carfilzomib is associated with less severe PN than bortezomib, however, the role of HtrA2 or other off target effects in proteasome inhibitor induced PN remains to be determined. 
IV.A.4. Bortezomib Efficacy in Solid Tumors. Despite bortezomib's clinical efficacy in treating hematological malignancies, it has had limited success in clinical trials for solid tumors. This may arise from poor bortezomib tissue penetration (at the doses used in $\mathrm{MM}$ ) and rapid clearance from the blood ${ }^{218}$. Dosage cannot be increased to overcome poor tissue penetration due to increased risk for PN. Second generation proteasome inhibitor are in development to overcome these limitations of bortezomib. Ongoing clinical trials are evaluating combinations of chemotherapy and radiotherapy with bortezomib in a search for new synergistic drug combinations.

\section{IV.B. Second Generation proteasome inhibitors}

Bortezomib treatment efficacy in human cancer renewed interest in developing other novel proteasome inhibitors to overcome bortezomib limitations. Two second generation proteasome inhibitors, carfilzomib (2012) and ixazomib (2015), are FDA approved for relapsed-refractory MM patients. Carfilzomib and ixazomib are well tolerated in heavily pretreated patients and show effectiveness in bortezomib-resistant cases ${ }^{211}$. Importantly, most cases of PN with carfilzomib and ixazomib are low grade and usually do not worsen preexisting PN resultant from previous bortezomib treatment ${ }^{211}$. Schlafer et al. (2017) provide a detailed review of the bortezomib, carfilzomib, and ixazomib clinical trials. Here we highlight second-generation FDA approved Pls and selected newer Pls undergoing preclinical evaluation in hematological cancers, solid tumors, and autoimmune disorders ${ }^{208}$. Our list of proteasome inhibitors is not intended to be comprehensive, but to rather increase familiarity with these important aspects. Table 4 lists important properties of first- and second-generation proteasome inhibitors in clinical trials.

IV.B.1. Carfilzomib. Carfilzomib (Kyprolis®) is the second FDA approved proteasome inhibitor. Carfilzomib is used as a single agent or in combination with immunomodulatory agents in RRMM patients who have received one to three prior therapies, including other proteasome inhibitors ${ }^{219}$. Carfilzomib has a tripeptide backbone containing phenylalanine, leucine, and homophenylalanine 
with a terminal epoxyketone group that forms an irreversible covalently bond with the proteasome catalytic threonine ${ }^{220}$. As with epoxomicin, the carfilzomib epoxyketone warhead forms a dual covalent adduct with the active site threonine, which greatly reduces the off targets.

Importantly, carfilzomib provides some therapeutic benefit for RRMM patients who relapse after bortezomib treatment, while also rendering less neurotoxic side effects ${ }^{211,219}$. The ENDEAVOR phase III clinical trial compared bortezomib plus dexamethasone versus carfilzomib plus dexamethasone in a cohort of newly diagnosed MM patients. ENDEAVOR reported significantly higher incidence of $\geq 2 \mathrm{PN}$ in the bortezomib group (32\%) vs. $6 \%$ in the carfilzomib group ${ }^{221}$. This was the first head to head comparison between bortezomib and carfilzomib patients. Unfortunately, drug resistance is observed following carfilzomib treatment in a small subset of patients ${ }^{222}$. Although the resistance mechanism is not fully elucidated, studies suggest that increased expression of drug efflux pump P-glycoprotein (P-gp, a known transporter of carfilzomib) in carfilzomib-resistant cells contributes to the resistant phenotype ${ }^{223}$. However, more detailed studies are needed to confirm this resistance mechanism.

Carfilzomib also shows therapeutic promise in several preclinical models of solid tumors. Carfilzomib effectively sensitized tumor cells to doxorubicin induced apoptosis in several in vivo preclinical solid tumor models including neuroblastoma and colon cancer ${ }^{224,225}$. Many doxorubicin (dox) resistant tumor cells have upregulated NF-kB activity that promotes survival 226,227,

\begin{tabular}{|c|c|c|c|c|c|c|c|c|}
\hline Inhibitor & Class & $\begin{array}{c}\mathrm{CT}-\mathrm{L} \\
1 C_{50}(n M)\end{array}$ & $\begin{array}{c}\mathrm{C}-\mathrm{L} \\
1 C_{50}(n M)\end{array}$ & $\begin{array}{c}\mathrm{T}-\mathrm{L} \\
1 C_{50}^{(n M)}\end{array}$ & $\begin{array}{l}\text { Half-life } \\
\text { (min) }\end{array}$ & Route & Binding & Ref \\
\hline Bortezomib & boronate & 7.9 & 53 & 590 & 110 & IVISC & $\begin{array}{l}\text { Slowly } \\
\text { reversible }\end{array}$ & Cauhan et al., 2005 \\
\hline Carfilzomib & epoxyketone & 6 & 2400 & 3600 & $<30$ & IV & Irreversible & Kuhn et al., 2007 \\
\hline Ixazomib & boronate & 3.4 & 31 & 3500 & 18 & Oral & Reversible & $\begin{array}{l}\text { Kupperman, et al., } 2010 \\
\text { Cauhan et al., } 2005\end{array}$ \\
\hline Marizomib & $\beta$-lactone & 3.5 & 430 & 28 & $10-15$ & IV & Irreversible & Feling et al., 2003 \\
\hline Oprozomib & epoxyketone & $\begin{array}{l}36(\beta 5) \\
82(\beta 5 i)\end{array}$ & ND & ND & $30-90$ & Oral & Irreversible & $\begin{array}{l}\text { Zhou et al., } 2009 \\
\text { Roccaro et al., } 2010\end{array}$ \\
\hline
\end{tabular}


suggesting combination treatment with a proteasome inhibitor may be effective at overcoming dox resistance. Although the exact mechanisms are unknown, it is thought that carfilzomibmediated NF-KB inhibition sensitizes tumor cells to doxorubicin. Based on these preclinical studies, carfilzomib (in combination with other chemotherapy drugs) is currently undergoing phase I and II clinical trials in advanced solid tumors, renal disease, transplant rejection, and hematological malignancies (clinicaltrials.gov).

IV.B.2. Ixazomib. Bortezomib and carfilzomib are administered intravenously, requiring patients to visit a clinic several times over the course of their treatment. Ixazomib (MLN9708, Ninlaro®) is the first and only orally bioavailable PI FDA approved for RRMM treatment ${ }^{228}$. Ixazomib was developed through a large-scale boron-containing $\mathrm{PI}$ screening for compounds with physiochemical properties distinct from bortezomib. Ixazomib is a bioavailable prodrug which is hydrolyzed into the active metabolite MLN2238 when exposed to the GI tract and plasma and is a potent and reversible $\beta 5$ proteasome subunit inhibitor ${ }^{229}$.

Despite similarities, there are important distinctions between ixazomib and bortezomib. Importantly, clinical trials report a lower incidence of $\mathrm{PN}$ in patients treated with ixazomib compared to bortezomib, and ixazomib is effective in treating bortezomib-resistant RRMM patients ${ }^{230}$. In addition, ixazomib demonstrated five times higher drug distribution supported by blood volume distribution ( $\mathrm{Vd}$ ), with a $\mathrm{Vd} 20.2 \mathrm{~L} / \mathrm{kg}$ for ixazomib vs. $\mathrm{Vd}=4.3 \mathrm{~L} / \mathrm{kg}$ for bortezomib 229. Ixazomib also displays anti-tumor efficacy in solid tumor preclinical models ${ }^{224}$. Numerous phase I and II clinical trials are underway, evaluating ixazomib treatment in glioblastomas, solid tumors, triple negative breast cancer, B cell lymphoma, lupus, bladder cancer, and lymphoma.

\section{IV.C. Additional proteasome inhibitors in clinical trials.}

IV.C.1. Marizomib. Marizomib (NPI-0052, Salinosporamide A) is a naturally occurring PI isolated from the marine actinomycete Salinispora tropica and is under development for MM and 
glioblastoma (GBM) treatment ${ }^{231}$. Marizomib is a $y$-lactam- $\beta$-lactone which demonstrates unique binding and bioavailability profiles, setting it apart from other Pls. Its unusual binding mechanism was elucidated using biochemical and crystallography approaches. In contrast to other $\beta$ lactones, marizomib has unique chloroethyl and cyclohex-2-enylcarbinol substituents, giving rise to important interactions within proteasome active sites. These unique interactions are thought to be responsible for marizomib's high proteasome affinity and specificity ${ }^{64}$.

Marizomib irreversibly inhibits $\beta 5\left(\mathrm{IC}_{50} 3.5 \mathrm{nM}\right)$ and $\beta 2\left(\mathrm{IC}_{50} 28 \mathrm{nM}\right)$ active sites, although it can inhibit $\beta 1$ at higher concentrations $\left(\mathrm{IC}_{50} 430 \mathrm{nM}\right)^{232}$. In vitro and binding competition experiments show marizomib binds to all three subunits at clinically relevant doses ${ }^{229}$. Unexpectedly, patient's $\beta 1$ and $\beta 2$ activities were not affected (in PBMCs) during the first cycle of marizomib treatment ${ }^{233}$. After the second dose cycle, decreased peptidase was observed, but only in packed whole blood samples - which contain mostly erythrocytes ${ }^{233}$. Whether marizomib inhibits $\beta 1$ and $\beta 2$ activity in nucleated cells that can synthesize new proteins remains to be determined. Importantly, marizomib shows the ability to overcome bortezomib and carfilzomib resistance in a limited number of RRMM patients ${ }^{233}$. Additional trials investigating marizomib in combination with other chemotherapy drugs in RRMM are ongoing.

Marizomib is the only PI in clinical trials that can permeate the blood brain barrier, making it an attractive candidate in CNS malignancy treatment. In animal studies, marizomib distributed to the brain at $30 \%$ blood levels in rats and significantly inhibited (>30\%) baseline chymotrypsin-like proteasome activity in monkey brain tissue ${ }^{234}$. Furthermore, marizomib treatment elicited a significant antitumor effect in a rodent intracranial malignant glioma model ${ }^{234}$ and was well tolerated in phase I/II clinical trials for advanced and newly diagnosed glioblastoma. Based on encouraging results from phase I and phase II marizomib trials in GBM patients, a phase III trial of marizomib in combination with standard temozolomide-based radiochemotherapy for patients 
with newly diagnosed GBM (clinical trial NCT03345095ํ) is scheduled to begin June 2018. GBM is the most common high-grade brain malignancy in adults with a $25 \%$ two-year survival after standard treatment ${ }^{235}$. New therapies are critically needed for these patients.

IV.C.2. Oprozomib. Efforts to synthesize an orally bioavailable epoxyketone proteasome inhibitor led to oprozomib (PR-047, ONX-0912) development. Oprozomib is an irreversible and potent $\beta 5 \mathrm{c}$ and $\beta 5$ i proteasome subunit inhibitor, with an IC50 $36 \mathrm{nM}$ and $82 \mathrm{nM}$, respectively ${ }^{236}$. Oprozomib oral administration demonstrated anti-tumor activity in MM, squamous cell carcinoma of the head and neck, and colorectal cancer preclinical models ${ }^{237}$.

The first phase I study (NCT01365559) with single-agent oprozomib evaluated patients with advanced solid tumors. Oprozomib was well tolerated, with low-grade gastrointestinal side effects being the most common. Unfortunately, despite dose-dependent increases in proteasome inhibition, the best response achieved was stable disease (23\% of patients) and no clinically meaningful correlates between proteasome inhibition and treatment efficacy were observed. Considering this was a heavily pretreated patient population with advanced cancer, the lack of a therapeutic response does not necessarily reflect the potential of oprozomib to treat earlier stages or other cancers. Clinical studies investigating the oprozomib efficacy in hematological malignancies are ongoing. In a phase I/II study (NCT01416428²), a new oral oprozomib formulation is being investigated, and other studies are investigating oprozomib in combination with dexamethasone and lenalidomide.

\section{IV.D. Immunoproteasome specific proteasome inhibitors}

Specifically targeting the immunoproteasome could be advantageous over currently approved Pls in treating certain diseases. The immunoproteasome is present at low levels in normal cells. In

\footnotetext{
${ }^{1}$ ClinicalTrials.gov [Internet]. Bethesda (MD): National Library of Medicine (US). 2017 Nov 17 -. Identifier NCT03345095

2 ClinicalTrials.gov [Internet]. Bethesda (MD): National Library of Medicine (US). 2011 Aug 5 -. Identifier NCT01416428
} 
contrast, some cancers (including MM), inflammatory diseases, and autoimmune diseases have increased immunoproteasome subunit expression ${ }^{238}$. It is thought that selective immunoproteasome inhibition may have less adverse effects than broadly acting Pls like bortezomib and carfilzomib since certain cell populations would be preferentially affected ${ }^{239}$.

IV.D.1. ONX-0914. ONX-0914 (PR-957) is an irreversible epoxyketone $\beta 5 \mathrm{i}$ immunoproteasome subunit inhibitor, with minimal cross-reactivity for the constitutive proteasome $\beta 5$ subunit $(\beta 5 c)$. Preclinical trials demonstrate $\beta 5$ i inhibition with ONX-0914 attenuates disease progression in colorectal cancer ${ }^{240}$, rheumatoid arthritis ${ }^{241}$, and systemic lupus erythematosus animal models 242. Importantly, ONX-0914 displays low neurotoxicity without sacrificing efficacy, an effect that may be attributed to the selective $\beta 5$ i inhibition ${ }^{243}$. Johnson et al. (2017) synthesized and screened ONX-0914 analogues for $\beta 2 \mathrm{i}$ inhibition. They identified and characterized compound KZR-504, a $\beta 2$ i selective proteasome inhibitor. However, KZR-504 displayed poor membrane permeability and did not ameliorate cytokine release from stimulated splenocytes ${ }^{244}$.

Another analogue, KZR-616, was well tolerated in a phase I clinical trial in healthy volunteers with minimal adverse side effects (Lickliter et al., $2017^{3}$ ). KZR-616 recently entered a phase Ib/II clinical trial (NCT03393013 ${ }^{4}$ ) as a single agent treatment for autoimmune-triggered inflammation (systemic lupus erythematosus).

\section{IV.E. Novel combination therapies}

Anti-cancer drugs are often administered in combination to synergistically enhance cytotoxicity and prevent drug-resistant tumor cell population development. Several known chemotherapy resistance mechanisms result from abrogated proteasome activity. For example, NF-kB activity

\footnotetext{
3 Lickliter J, Anderl J, Kirk CJ, Wang J, Bomba D. KZR-616, a Selective Inhibitor of the Immunoproteasome, Shows a Promising Safety and Target Inhibition Profile in a Phase I, Double-Blind, Single (SAD) and Multiple Ascending Dose (MAD) Study in Healthy Volunteers[abstract].ArthritisRheumatol. 2017; 69(suppl 10

${ }^{4}$ ClinicalTrials.gov [Internet]. Bethesda (MD): National Library of Medicine (US). 2018 Jan 8 -. Identifier NCT03393013
} 
is upregulated in solid tumors that develop chemotherapy (e.g. doxorubicin) or radiation resistance and is a driving force behind the resistant phenotype ${ }^{51}$. Accordingly, preclinical studies with solid-tumor xenograft and cellular models show increased sensitivity to chemotherapy and/or radiation induced apoptosis when combined with proteasome inhibitors ${ }^{245}$. Several phase I and II clinical trials are evaluating PI safety and efficacy in combination with various chemotherapy agents in recurrent or refractory advanced cancers.

$\beta 1$ and $\beta 2$ site upregulation has been reported in tumor cells resistant to bortezomib or carfilzomib treatment ${ }^{51}$. LU-102 is a peptide vinyl-sulfone $\beta 2$ specific inhibitor ${ }^{246}$ and alone it is toxic to MM cells, but it also synergized with $\beta 5$ inhibitors (bortezomib and carfilzomib) to overcome PI resistance in $\mathrm{MM}$ cell lines ${ }^{246}$. The observed synergy between $\beta 5$ and $\beta 2$ inhibitors suggests that multiple site-specific proteasome inhibitor combinations may be an effective alternative in $\mathrm{PI}$ resistant malignancies with reduced risk of adverse side effects.

\section{Novel Proteasome Drug Targets}

\section{V.A. Deubiquitinating enzyme inhibitors}

With three ubiquitin receptors on the proteasome, several shuttling factors, and multiple ubiquitin chain types on substrates, the process of substrate recognition by the $26 \mathrm{~S}$ proteasome is incredibly complex and much remains unknown. We briefly summarized the process of ubiquitindegradation in Part I and here, will discuss how deubiquitinating enzymes (DUBs) may be targeted to treat diseases. Detailed reviews of $26 \mathrm{~S}$ substrate recognition and processing have already been written ${ }^{77}$ and ${ }^{76}$.

Three DUBs are associated with the 26S proteasome: Rpn11, Uch37 and Usp14. Rpn11 is the only DUB that is an integral part of the 195 regulatory particle. Positioned directly above the translocation pore, Rpn11 is a metalloprotease that removes $\mathrm{Ub}$ chains at their attachment point 
(lysine) on the substrate that is committed for degradation ${ }^{75}$. Mutations in Rpn11 that disrupt its catalytic activity stall ubiquitin substrate degradation and eventually lead to cell death ${ }^{247}$. Li et al. (2017) recently developed capzimin, a first-in-class selective inhibitor of Rpn11. The Rpn11 active site is located within the highly conserved JAMM motif and features a catalytic $\mathrm{Zn}^{2+}$ ion ${ }^{247}$. Capzimin binds the catalytic zinc ion and prevents Rpn11 activity ${ }^{248}$. Remarkably, Capzimin treatment stabilized proteasome substrates and blocked proliferation in several tumor cell lines 248. This anti-tumor activity suggests Rpn11 inhibition may be an effective alternative to active site inhibition for treating malignancies. Unlike Rpn11, Uch37 and Usp14 are only transiently associated with the 195 regulatory particle ${ }^{166,249}$. Uch37 and Usp14 enzymes trim Ub chains before the substrate is committed to degradation, which may suppress protein degradation by promoting early substrate release ${ }^{76}$. Accordingly, loss of Usp14 homologue Ubp6 increases substrate degradation by the proteasome ${ }^{250,251}$. Despite high affinity, Usp14 easily dissociates from 26S complexes during conventional proteasome purification techniques and should be considered when designing in vitro experiments ${ }^{76}$.

Enhancing proteasome function has the potential to treat protein misfolding disorders, such as neurodegenerative diseases, and Usp14 inhibition may promote ubiquitin-dependent protein degradation. To this end, Boselli et al. (2017) developed IU-47, a potent and specific inhibitor of Usp14. Inhibition of Usp14 DUB activity by IU-47 promoted proteasome degradation of the microtubule associated protein tau (implicated in the pathogenesis of Alzheimer's disease) in neuronal cultures and enhanced resistance to oxidative stress ${ }^{252}$. The implications of pharmacological proteasome activation will be discussed in the following section.

\section{V.B. Activation of the $20 \mathrm{~S}$ by gate-opening}

We highlighted essential functions of the proteasome required for proper neuronal functioning in Part I. Then Part II discussed the negative impacts of proteasome inhibition on primary sensory 
neurons. To bridge these concepts, we will further discuss evidence that supports the proteasome as a target for pharmacological activation to treat proteopathies.

The most common neurodegenerative diseases are characterized by an accumulation of aggregation-prone proteins concomitant with a loss of proteostasis, which results in progressive death of neurons ${ }^{121-123}$. Impaired proteasome function has been implicated, as a primary cause or a secondary consequence, in the pathogenesis of many neurodegenerative diseases, including Alzheimer's, Parkinson's, and Huntington's disease ${ }^{47,124-127 . ~}$

Soluble forms of aggregated proteins, called oligomers, are implicated in the pathogenesis of most neurodegenerative diseases ${ }^{253,254}$ and haven been shown to impair proteasome function 255-260. Our lab recently identified a mechanism in which soluble oligomers of different proteins from multiple neurodegenerative diseases allosterically impair the proteasome gate by a shared mechanism ${ }^{175}$. These toxic oligomers shared a similar three-dimensional conformation recognized by the anti-oligomer antibody, $\mathrm{A} 11^{261}$. The $\mathrm{A} 11+$ oligomers bound to the outside of the 205 cylinder and stabilized the gate in the closed conformation. However, proteasome function could be rescued by adding an 8 residue HbYX peptides. The HbYX peptides allosterically open the gate and the $\mathrm{HbYX}$ motif peptides overcame the oligomer inhibition and restored proteasome function ${ }^{175}$.

A recent and popular idea for treating neurodegenerative diseases is enhancing proteasome activity to suppress toxicity and related proteotoxic pathophysiology, but to date no drugs are available that directly activate proteasome function.

Since IU-47 exerts its effect upstream of the proteasome gate, it is not expected to function in conditions where the proteasome gate is impaired. Choi et al. (2016) generated a HEK293 cell line with a stable transfection of a mutant $20 S \alpha$-subunit $(\alpha 3 \Delta N)$ that induces $20 S$ gate opening. They showed that HEK293- $\alpha 3 \Delta \mathrm{N}$ cells had increased degradation of proteasome substrates 
(including tau protein) and increased resistance to oxidative stress compared to WT ${ }^{171}$. While serving as a proof-of-principle that opening the proteasome gate can clear aggregation prone proteins and increase cell viability, it cannot be determined if pharmacologically activating the proteasome in an already diseased state (pre-existing oligomers, aggregates, and oxidative stress) can restore cellular proteostasis. Nonetheless, elucidation of a common mechanisms of proteasome inhibition is a major step towards the rational design of proteasome activating compounds, which may be a promising route to restore proteostasis in diseases.

\section{Conclusions}

As a highly regulated, multicatalytic macromolecular complex, the proteasome possesses multiple drug targets to module its degradation capacity. Proteasome inhibitors helped early researchers study the proteasome's cellular functions. Today, three inhibitors are approved for use in the clinic to treat hematological cancers and several more inhibitors (synthetic and naturally occurring) are in preclinical and clinical testing. Fine tuning the pharmacological properties of proteasome inhibitors may improve their efficacy for use in solid tumors. The last decade has witnessed major progress in our understanding of 265 proteasome structure and dynamics. In the coming years, we expect development of new inhibitors and activators of nonproteolytic components of the $26 \mathrm{~S}$ proteasome. We anticipate that proteasome activation will become a validated target to treat proteotoxic diseases.

\section{Acknowledgements}

We are grateful to Steven Markwell for critical reading of the manuscript.

\section{Author Contributions}

Wrote the manuscript: Tiffany Thibaudeau with the assistance of David M. Smith 


\section{References}

1. Schoenheimer, R., Ratner, S. \& Rittenberg, D. Studies in protein metabolism: VII. The metabolism of tyrosine. J Biol Chem 127, 333-344 (1939).

2. Schoenheimer, R. The dynamic state of body constituents. Cancer Research 2, (Harvard University Press, 1942).

3. Lecker, S. H., Goldberg, A. L. \& Mitch, W. E. Protein degradation by the ubiquitinproteasome pathway in normal and disease states. J. Am. Soc. Nephrol. 17, 1807-1819 (2006).

4. Glickman, M. H. \& Ciechanover, A. The ubiquitin-proteasome proteolytic pathway: Destruction for the sake of construction. Physiol Rev 82, 373-428 (2002).

5. Duve, C. de, Gianetto, R., Appelmans, F. \& Wattiaux, R. Enzymic content of the mitochondria fraction. Nature 172, 1143-1144 (1953).

6. Appelmans, F., Wattiaux, R. \& De Duve, C. Tissue fractionation studies: the association of acid phosphatase with a special class of cytoplasmic granules in rat liver. Biochem $J$ 59, 438-445 (1954).

7. de Duve, C., Pressman, B. C., Gianetto, R., Wattiaux, R. \& Appelmans, F. Tissue fractionation studies. 6 . Intracellular distribution patterns of enzymes in rat-liver tissue. Biochem. J. 60, 604-617 (1955).

8. de Duve, C. \& Wattiaux, R. Functions of lysosomes. Annu. Rev. Physiol. 28, 435-492 (1966).

9. Simpson, M. V. The release of labeled amino acids from the proteins of rat liver slices. $J$ Biol Chem 201, 143-154 (1953).

10. Mandelstam, J. Turnover of protein in growing and non-growing populations of Escherichia coli. Biochem. Journal1 69, 110-119 (1958).

11. Schimke, R. The importance of both synthesis and degradation in the control of arginase levels in rat liver. J Biol Chem 239, 3808-3817 (1964).

12. Schimke, R. T. \& Doyle, D. Control of enzyme levels in animal tissues. Annu. Rev. Biochem. 39, 929-76 (1970).

13. Zavortink, M., Thacher, T. \& Rechsteiner, M. Degradation of proteins microinjected into cultured mammalian cells. J. Cell. Physiol. 100, 175-185 (1979).

14. Goldberg, A. L. Degradation of abnormal proteins in Escherichia coli. Proc. Natl. Acad. Sci. 69, 422-426 (1972).

15. Goldberg, A. L. \& Dice, J. F. Intracellular protein degradation in mammalian and bacterial cells. Annu. Rev. Biochem. 43, 835-869 (1974).

16. Poole, B., Ohkuma, S. \& Warburton, M. The accumulation of weakly basic substances in lysosomes and the inhibition of intracellular protein degradation. Acta Biol. Med. Germ. 36, 1777-1788 (1977).

17. Poole, B., Ohkuma, S. \& Warburton, M. Selective control of the degradation of normal and aberrant proteins in Reuber H35 hepatoma cells. Biochem. J. 156, 609-617 (1976).

18. Knowles, S. E., Gunn, J. M., Hanson, R. W. \& Ballard, F. J. Increased degradation rates of protein synthesized in hepatoma cells in the presence of amino acid analogues.

Biochem. J. 146, 595-600 (1975).

19. Knowles, S. E. \& Ballard, F. J. Selective control of the degradation of normal and aberrant proteins in Reuber H35 hepatoma cells. Biochem. J. 156, 609-17 (1976).

20. Goldberg, A. L. \& St John, A. C. Intracellular protein degradation in mammalian and bacterial cells. Annu. Rev. Biochem. 45, 747-803 (1976).

21. Etlinger, J. D. \& Goldberg, A. L. A soluble ATP-dependent proteolytic system responsible for the degradation of abnormal proteins in reticulocytes. Proc. Natl. Acad. Sci. 74, 54-58 (1977).

22. Bigelow, S., Hough, R. \& Rechsteiner, M. The selective degradation of injected proteins 
occurs principally in the cytosol rather than in lysosomes. Cell 25, 83-93 (1981).

23. Wilk, S. \& Orlowski, M. Cation-sensitive neutral endopeptidase: isolation and specificity of the bovine pituitary enzyme. J. Neurochem. 35, 1172-1182 (1980).

24. Wilk, S. \& Orlowski, M. Evidence that pituitary cation-sensitive neutral endopeptidase is a multicatalytic protease complex. J. Neurochem. 40, 842-849 (1983).

25. Tanaka, K. et al. Proteasomes (multi-protease complexes) as $20 \mathrm{~S}$ ring-shaped particles in a variety of eukaryotic cells. J Biol Chem 263, 16209-17 (1988).

26. Arrigo, A. P., Tanaka, K., Goldberg, A. L. \& Welch, W. J. Identity of the 19S 'prosome' particle with the large multifunctional protease complex of mammalian cells (the proteasome). Nature 331, 192-194 (1988).

27. Goldberg, A. L. Functions of the proteasome: from protein degradation and immune surveillance to cancer therapy. Biochem. Soc. Trans. 35, 12-17 (2007).

28. Ciechanover, A., Hod, Y. \& Hershko, A. A heat-stable polypeptide component of an ATPdependent proteolytic system from reticulocytes. Biochem. Biophys. Res. Commun. 81, 1100-1105 (1978).

29. Wilkinson, K. D., Urban, M. K. \& Haas, A. L. Ubiquitin is the ATP-dependent proteolysis factor I of rabbit reticulocytes. J. Biol. Chem. 255, 7529-7532 (1980).

30. Ciechanover, A., Heller, H., Elias, S., Haas, A. L. \& Hershko, A. ATP-dependent conjugation of reticulocyte proteins with the polypeptide required for protein degradation. Proc. Natl. Acad. Sci. 77, 1365-1368 (1980).

31. Hershko, A., Ciechanover, A., Heller, H., Haas, A. L. \& Rose, I. A. Proposed role of ATP in protein breakdown: Conjugation of protein with multiple chains of the polypeptide of ATP-dependent proteolysis. Proc. Natl. Acad. Sci. 77, 1783-1786 (1980).

32. Tanaka, K., Waxman, L. \& Goldberg, A. L. ATP serves two distinct roles in protein degradation in reticulocytes, one requiring and one independent of ubiquitin. J. Cell Biol. 96, 1580-1585 (1983).

33. Hough, R., Pratt, G. \& Rechsteiner, M. Ubiquitin-lysozyme conjugates. Identification and characterization of an ATP-dependent protease from rabbit reticulocyte lysates. J. Biol. Chem. 261, 2400-2408 (1986).

34. Hough, R., Pratt, G. \& Rechsteiner, M. Purification of two high molecular weight proteases from rabbit reticulocyte lysate. J. Biol. Chem. 262, 8303-8313 (1987).

35. Hershko, A., Heller, H., Elias, S. \& Ciechanover, A. Components of ubiquitin-protein ligase system: Resolution, affinity purification and role in protein breakdown. J Biol Chem 258, 8206-8214 (1983).

36. Ciechanover, A. Proteolysis: from the lysosome to ubiquitin and the proteasome. Nat Rev Mol Cell Biol 6, 79-87 (2005).

37. Ciechanover, A., Elias, S., Heller, H. \& Hershko, A. Covalent affinity purification of ubiquitin-activating enzyme. J Biol Chem 257, 2537-2542 (1982).

38. Ciechanover, A. Intracellular protein degradation: from a vague idea through the lysosome and the ubiquitin-proteasome system and onto human diseases and drug targeting. Bioorganic Med. Chem. 21, 3400-3410 (2017).

39. Finley, D., Ciechanover, A. \& Varshavsky, A. Thermolability of ubiquitin-activating enzyme from the mammalian cell cycle mutant ts85. Cell 37, 43-55 (1984).

40. Ciechanover, A., Finley, D. \& Varshavsky, A. Ubiquitin dependence of selective protein degradation demonstrated in the mammalian cell cycle mutant ts85. Cell 37, 57-66 (1984).

41. Hershko, A., Eytan, E., Ciechanover, A. \& Haas, A. L. Immunochemical analysis of the turnover of ubiquitin-protein conjugates in intact cells: Relationship to the breakdown of abnormal proteins. J. Biol. Chem. 257, 13964-13970 (1982).

42. Peters, J. M. The anaphase-promoting complex: Proteolysis in mitosis and beyond. Mol. Cell 9, 931-943 (2002). 
43. Bassermann, F., Eichner, R. \& Pagano, M. The ubiquitin proteasome system Implications for cell cycle control and the targeted treatment of cancer. UbiquitinProteasome Syst. 1843, 150-162 (2014).

44. Hershko, A. \& Ciechanover, A. The ubiquitin system. Annu. Rev. Biochem. 67, 425-479 (1998).

45. Ben-Neriah, Y. Regulatory functions of ubiquitination in the immune system. Nat. Immunol. 3, 20-26 (2002).

46. Sakamoto, K. M. Ubiquitin-dependent proteolysis: Its role in human diseases and the design of therapeutic strategies. Mol. Genet. Metab. 77, 44-56 (2002).

47. McKinnon, C. \& Tabrizi, S. J. The ubiquitin-proteasome system in neurodegeneration. Antioxid. Redox Signal. 5, 2302-2321 (2014).

48. Layfield, R., Lowe, J. \& Bedford, L. The ubiquitin-proteasome system and neurodegenerative disorders. Essays Biochem. 41, 157-71 (2005).

49. Dou, Q. P., Smith, D. M., Daniel, K. G. \& Kazi, A. Interruption of tumor cell cycle progression through proteasome inhibition: Implications for cancer therapy. Prog Cell Cycle Res 5, 441-446 (2003).

50. Schmidt, M. \& Finley, D. Regulation of proteasome activity in health and disease. Biochim. Biophys. Acta 1843, 13-25 (2014).

51. Kisselev, A. F. \& Goldberg, A. L. Proteasome inhibitors: From research tools to drug candidates. Chem. Biol. 8, 739-758 (2001).

52. DeMartino, G. N. et al. Identification, purification, and characterization of a PA700dependent activator of the proteasome. J. Biol. Chem. 271, 3112-3118 (1996).

53. Adams, G. M., Crotchett, B., Slaughter, C. A., DeMartino, G. N. \& Gogol, E. P. Formation of proteasome-PA700 complexes directly correlates with activation of peptidase activity. Biochemistry 37, 12927-1 2 (1998).

54. DeMartino, G. N. \& Slaughter, C. A. The proteasome, a novel protease regulated by multiple mechanisms. J Biol Chem 274, 22123-22126 (1999).

55. Voges, D., Zwickl, P. \& Baumeister, W. The 26S proteasome: A molecular machine designed for controlled proteolysis. Annu. Rev. Biochem. 68, 1015-1068 (1999).

56. Groll, M. et al. Structure of $20 \mathrm{~S}$ proteasome from yeast At $2.4 \AA ̊$ resolution. Nature 386, 463-471 (1997).

57. Groll, M. et al. A gated channel into the proteasome core particle. Nat. Struct. Biol. 7, 1062-1067 (2000).

58. Köhler, A. et al. The axial channel of the proteasome core particle is gated by the Rpt2 ATPase and controls both substrate entry and product release. Mol. Cell 7, 1143-1152 (2001).

59. Ruschak, A. M. \& Kay, L. E. Proteasome allostery as a population shift between interchanging conformers. Proc. Natl. Acad. Sci. 109, E3454-62 (2012).

60. Religa, T. L., Sprangers, R. \& Kay, L. E. Dynamic regulation of archaeal proteasome gate opening as studied by TROSY NMR. Science 328, 98-102 (2010).

61. Finley, D., Chen, X. \& Walters, K. J. Gates, channels, and switches: Elements of the proteasome machine. Trends Biochem. Sci. 41, 77-93 (2016).

62. Borissenko, L. \& Groll, M. 20S proteasome and its inhibitors: crystallographic knowledge for drug development. Chem. Rev. 107, 687-717 (2007).

63. Arendt, C. S. \& Hochstrasser, M. Identification of the yeast $20 \mathrm{~S}$ proteasome catalytic centers and subunit interactions required for active-site formation. Proc. Natl. Acad. Sci. U. S. A. 94, 7156-7161 (1997).

64. Groll, M. \& Huber, R. Inhibitors of the eukaryotic 20 S proteasome core particle: A structural approach. Biochim. Biophys. Acta 1695, 33-44 (2004).

65. Tanaka, K. Role of proteasomes modified by interferon- $\gamma$ in antigen processing. $J$. Leukoc. Biol. 56, 571-575 (1994). 
66. Rock, K. L. \& Goldberg, A. L. Degradation of cell proteins and the generation of MHC class I-presented peptides. Annu Rev Immunol 17, 739-779 (1999).

67. Van Kaert, L. et al. Altered peptidase and viral-specific T cell response in LMP2 mutant mice. Immunity 1, 533-541 (1994).

68. Gaczynska, M., Rock, K. L. \& Goldberg, A. L. Gamma-interferon and expression of MHC genes regulate peptide hydrolysis by proteasomes. Nature 365, 264-267 (1993).

69. Falk, K. \& Rotzschke, O. Consensus motifs and peptide ligands of MHC class I molecules. Semin Immunol. 5, 81-94 (1993).

70. Kniepert, A. \& Groettrup, M. The unique functions of tissue-specific proteasomes. Trends Biochem. Sci. 39, 17-24 (2014).

71. Murata, S. et al. Regulation of CD8+ T cell development by thymus-specific proteasomes. Science 316, 1349-1354 (2007).

72. Sledz, P. et al. Structure of the 26S proteasome with ATP-yS bound provides insights into the mechanism of nucleotide-dependent substrate translocation. Proc. Natl. Acad. Sci. 110, 7264-7269 (2013).

73. Bashore, C. et al. Ubp6 deubiquitinase controls conformational dynamics and substrate degradation of the 26S proteasome. Nat. Struct. Mol. Biol. 22, 712-719 (2015).

74. Beck, F. et al. Near-atomic resolution structural model of the yeast $26 \mathrm{~S}$ proteasome. Proc. Natl. Acad. Sci. 109, 14870-14875 (2012).

75. Matyskiela, M. E., Lander, G. C. \& Martin, A. Conformational switching of the $26 \mathrm{~S}$ proteasome enables substrate degradation. Nat. Struct. Mol. Biol. 20, 781-788 (2013).

76. de Poot, S. A. H., Tian, G. \& Finley, D. Meddling with Fate: The Proteasomal Deubiquitinating Enzymes. J. Mol. Biol. 429, 3525-3545 (2017).

77. Collins, G. A. \& Goldberg, A. L. The logic of the $26 \mathrm{~S}$ proteasome. Cell 169, 792-806 (2017).

78. Peth, A., Kukushkin, N., Bossé, M. \& Goldberg, A. L. Ubiquitinated proteins activate the proteasomal ATPases by binding to Usp14 or Uch37 homologs. J. Biol. Chem. 288, 7781-7790 (2013).

79. Peth, A., Besche, H. C. \& Goldberg, A. L. Ubiquitinated proteins activate the proteasome by binding to Usp14/Ubp6, which causes $20 S$ gate opening. Mol. Cell 36, 794-804 (2009).

80. Peth, A., Uchiki, T. \& Goldberg, A. L. ATP-Dependent steps in the binding of ubiquitin conjugates to the 26s proteasome that commit to degradation. Mol. Cell 40, 671-681 (2010).

81. Snoberger, A., Anderson, R. T. \& Smith, D. M. The proteasomal ATPases use a slow but highly processive strategy to unfold proteins. Front. Mol. Biosci. 4, 18 (2017).

82. Rabl, J. et al. Mechanism of gate opening in the $20 \mathrm{~S}$ proteasome by the proteasomal ATPases. Mol. Cell 30, 360-368 (2008).

83. Yu, Y. et al. Interactions of PAN's C-termini with archaeal $20 \mathrm{~S}$ proteasome and implications for the eukaryotic proteasome-ATPase interactions. EMBO J. 29, 692-702 (2010).

84. Smith, D. M. et al. Docking of the proteasomal ATPases' carboxyl termini in the $20 \mathrm{~S}$ proteasome's $\alpha$-ring opens the gate for substrate entry. Mol. Cell 27, 731-744 (2007).

85. Smith, D. M., Benaroudj, N. \& Goldberg, A. Proteasomes and their associated ATPases: A destructive combination. J. Struct. Biol. 156, 72-83 (2006).

86. Beckwith, R., Estrin, E., Worden, E. J. \& Martin, A. Reconstitution of the 26S proteasome reveals functional asymmetries in its AAA+ unfoldase. Nat. Struct. Mol. Biol. 20, 11641172 (2013).

87. Johnston, S. C., Whitby, F. G., Realini, C., Rechsteiner, M. \& Hill, C. P. The proteasome $11 \mathrm{~S}$ regulator subunit REG alpha (PA28 alpha) is a heptamer. Protein Sci. 6, 2469-2473 (1997). 
88. Rechsteiner, M. \& Hill, C. P. Mobilizing the proteolytic machine: Cell biological roles of proteasome activators and inhibitors. Trends Cell Biol. 15, 27-33 (2005).

89. Baldin, V. et al. A novel role for PA28gamma-proteasome in nuclear speckle organization and SR protein trafficking. Mol. Biol. Cell 19, 1706-16 (2008).

90. Pickering, A. M. \& Davies, K. J. A. Differential roles of proteasome and immunoproteasome regulators $\mathrm{Pa} 28 \alpha \beta, \mathrm{Pa} 28 y$ and $\mathrm{Pa} 200$ in the degradation of oxidized proteins. Arch. Biochem. Biophys. 523, 181-190 (2012).

91. Khor, B. et al. Proteasome activator PA200 is required for normal spermatogenesis. Mol. Cell. Biol. 26, 2999-3007 (2006).

92. Ustrell, V., Hoffman, L., Pratt, G. \& Rechsteiner, M. Pa200, a nuclear proteasome activator involved in DNA repair. EMBO J. 21, 3516-3525 (2002).

93. Blickwedehl, J. et al. The proteasome activator PA200 regulates tumor cell responsiveness to glutamine and resistance to ionizing radiation. Mol. Cancer Res. 10, 937-944 (2012).

94. Sadre-Bazzaz, K., Whitby, F. G., Robinson, H., Formosa, T. \& Hill, C. P. Structure of a Blm10 complex reveals common mechanisms for proteasome binding and gate opening. Mol. Cell 37, 728-735 (2010).

95. Schmidt, M. et al. The HEAT repeat protein BIm10 regulates the yeast proteasome by capping the core particle. Nat Struct Mol Biol 12, 294-303 (2005).

96. Ortega, J. et al. The axial channel of the $20 \mathrm{~S}$ proteasome opens upon binding of the PA200 activator. J. Mol. Biol. 346, 1221-1227 (2005).

97. Witkowska, J. et al. Crystal structure of a low molecular weight activator Blm-pep with yeast 20 S proteasome - Insights into the enzyme activation mechanism. Sci. Rep. 7, 6177 (2017).

98. Qian, M. X. M. et al. Acetylation-mediated proteasomal degradation of core histones during DNA repair and spermatogenesis. Cell 153, 1012-1024 (2013).

99. Adams, J. et al. Proteasome inhibitors: a novel class of potent and effective antitumor agents. Cancer Res 59, 2615-2622 (1999).

100. Shah, S. A. et al. $26 \mathrm{~S}$ proteasome inhibition induces apoptosis and limits growth of human pancreatic cancer. J Cell Biochem 82, 110-122 (2001).

101. Dietrich, C., Bartsch, T., Schanz, F., Oesch, F. \& Wieser, R. J. p53-dependent cell cycle arrest induced by $\mathrm{N}$-acetyl-L-leucinyl-L-leucinyl-L-norleucinal in platelet-derived growth factor-stimulated human fibroblasts. Proc Natl Acad Sci 93, 10815-10819 (1996).

102. Machiels, B. M. et al. Detailed analysis of cell cycle kinetics upon proteasome inhibition. Cytometry 28, 243-252 (1997).

103. Wu, Y., Luo, H., Kanaan, N. \& Wu, J. The proteasome controls the expression of a proliferation-associated nuclear antigen Ki-67. J. Cell. Biochem. 76, 596-604 (2000).

104. Dulić, V. et al. p53-dependent inhibition of cyclin-dependent kinase activities in human fibroblasts during radiation-induced G1 arrest. Cell 76, 1013-1023 (1994).

105. King, R. W., Deshaies, R. J., Peters, J. M. \& Kirschner, M. W. How proteolysis drives the cell cycle. Science 274, 1652-1659 (1996).

106. Pagano, M. et al. Role of the ubiquitin-proteasome pathway in regulating abundance of the cyclin-dependent kinase inhibitor p27. Science 269, 682-685 (1995).

107. Chen, C., Edelstein, L. C. \& Gelinas, C. The Rel/NF-kB family directly activates expression of the apoptosis inhibitor Bcl-xL. Mol. Cell. Biol. 20, 2687-2695 (2000).

108. Wang, C. Y., Mayo, M. W., Korneluk, R. G., Goeddel, D. V. \& Baldwin, A. S. NF-KkappaB antiapoptosis: Induction of TRAF1 and TRAF2 and c-IAP1 and c-IAP2 to suppress caspase-8 activation. Science 281, 1680-1683 (1998).

109. Zong, W. X., Edelstein, L. C., Chen, C., Bash, J. \& Gélinas, C. The prosurvival Bcl-2 homolog Bfl-1/A1 is a direct transcriptional target of NF-KB that blocks TNFa-induced apoptosis. Genes Dev. 13, 382-387 (1999). 
110. Baldwin, A. S. Control of oncogenesis and cancer therapy resistance by the transcription factor NF-kB. J. Clin. Invest. 107, 241-246 (2001).

111. Scherer, D. C., Brockman, J. A., Chen, Z., Maniatis, T. \& Ballard, D. W. Signal-induced degradation of I kappa B alpha requires site-specific ubiquitination. Proc. Natl. Acad. Sci. 92, 11259-11263 (1995).

112. Chen, Z. et al. Signal-induced site-specific phosphorylation targets I kappa B alpha to the ubiquitin-proteasome pathway. Genes Dev 9, 1586-1597 (1995).

113. Spencer, E., Jiang, J. \& Chen, Z. J. Signal-induced ubiquitination of IkappaBalpha by the F-box protein Slimb/beta-TrCP. Genes Dev. 13, 284-94 (1999).

114. Winston, J. T. et al. The SCF( $\beta$-TRCP)-ubiquitin ligase complex associates specifically with phosphorylated destruction motifs in IKBa and $\beta$-catenin and stimulates IKBa ubiquitination in vitro. Genes Dev. 13, 270-283 (1999).

115. Wang, C. Y., Mayo, M. W. \& Baldwin, A. S. TNF- and cancer therapy-induced apoptosis: potentiation by inhibition of NF-kappaB. Science 274, 784-787 (1996).

116. Tai, H.-C. \& Schuman, E. M. Ubiquitin, the proteasome and protein degradation in neuronal function and dysfunction. Nat. Rev. Neurosci. 9, 826-38 (2008).

117. Aso, E. et al. Amyloid generation and dysfunctional immunoproteasome activation with disease progression in animal model of familial Alzheimer's disease. Brain Pathol. 22, 636-53 (2012).

118. Djakovic, S. N. et al. Phosphorylation of Rpt6 regulates synaptic strength in hippocampal neurons. J. Neurosci. 32, 5126-5131 (2012).

119. Fonseca, R., Vabulas, R. M., Hartl, F. U., Bonhoeffer, T. \& Nägerl, U. V. A balance of protein synthesis and proteasome-dependent degradation determines the maintenance of LTP. Neuron 52, 239-245 (2006).

120. Ramachandran, K. V \& Margolis, S. S. A mammalian nervous-system-specific plasma membrane proteasome complex that modulates neuronal function. Nat. Struct. Mol. Biol. 24, 419-430 (2017).

121. Brettschneider, J., Tredici, K. D., Lee, V. M. \& Trojanowski, J. Q. Spreading of pathology in neurodegenerative diseases: a focus on human studies. Nat. Rev. Neurosci. 16, 10920 (2015).

122. Rubinsztein, D. C. The roles of intracellular protein-degradation pathways in neurodegeneration. Nature 443, 780-786 (2006).

123. Selkoe, D. J. Folding proteins in fatal ways. Nature 426, 900-904 (2003).

124. Ciechanover, A. \& Brundin, P. The ubiquitin proteasome system in neurodegenerative diseases: Sometimes the chicken, sometimes the egg. Neuron 40, 427-446 (2003).

125. Keller, J. N., Hanni, K. B. \& Markesbery, W. R. Impaired proteasome function in Alzheimer's disease. J. Neurochem. 75, 436-439 (2000).

126. McNaught, K. S., Olanow, C. W., Halliwell, B., Isacson, O. \& Jenner, P. Failure of the ubiquitin-proteasome system in Parkinson's disease. Nat. Rev. Neurosci. 2, 589-594 (2001).

127. Ortega, Z., Díaz-Hernández, M. \& Lucas, J. J. Is the ubiquitin-proteasome system impaired in Huntington's disease? Cell. Mol. Life Sci. 64, 2245-2257 (2007).

128. Bedford, L. et al. Depletion of $26 \mathrm{~S}$ proteasomes neurodegeneration and lewy-like inclusions resembling human pale bodies. J. Neurosci. 28, 8189-8198 (2008).

129. $\mathrm{Li}, \mathrm{X}$. et al. Inhibiting the ubiquitin-proteasome system leads to preferential accumulation of toxic N-terminal mutant huntingtin fragments. Hum. Mol. Genet. 19, 2445-2455 (2010).

130. McNaught, K. S. et al. Impairment of the ubiquitin-proteasome system causes dopaminergic cell death and inclusion body formation in ventral mesencephalic cultures. J. Neurochem. 81, 301-306 (2002).

131. McNaught, K. S., Perl, D. P., Brownell, A. L. \& Olanow, C. W. Systemic exposure to proteasome inhibitors causes a progressive model of Parkinson's disease. Ann. Neurol. 
56, 149-162 (2004).

132. Wu, X. \& Rapoport, T. A. Mechanistic insights into ER-associated protein degradation. Curr Opin Cell Biol 53, 22-28 (2018).

133. Carvalho, P., Goder, V. \& Rapoport, T. A. Distinct ubiquitin-ligase complexes define convergent pathways for the degradation of ER proteins. Cell 126, 361-373 (2006).

134. Vashist, S. \& Ng, D. T. W. Misfolded proteins are sorted by a sequential checkpoint mechanism of ER quality control. J. Cell Biol. 165, 41-52 (2004).

135. Huyer, G. et al. Distinct machinery is required in Saccharomyces cerevisiae for the endoplasmic reticulum-associated degradation of a multispanning membrane protein and a soluble luminal protein. J. Biol. Chem. 279, 38369-38378 (2004).

136. Foresti, O., Rodriguez-Vaello, V., Funaya, C. \& Carvalho, P. Quality control of inner nuclear membrane proteins by the Asi complex. Science 346, 751-755 (2014).

137. Khmelinskii, A. et al. Protein quality control at the inner nuclear membrane. Nature 516, 410-413 (2014).

138. Bays, N. W., Wilhovsky, S. K., Goradia, A., Hodgkiss-Harlow, K. \& Hampton, R. Y. HRD4/NPL4 is required for the proteasomal processing of ubiquitinated ER proteins. Mol. Biol. Cell 12, 4114-28 (2001).

139. Braun, S. \& Matuschewski, K. Role of the ubiquitin-selective CDC48UFD1/NPL4 chaperone (segregase) in ERAD of OLE1 and other substrates. EMBO J. 21, 615-621 (2002).

140. Jarosch, E. et al. Protein dislocation from the ER requires polyubiquitination and the AAAATPase Cdc48. Nat Cell Biol 4, 134-139 (2002).

141. Rabinovich, E., Kerem, A., Frohlich, K.-U., Diamant, N. \& Bar-Nun, S. AAA-ATPase p97/Cdc48p, a cytosolic chaperone required for endoplasmic reticulum-associated protein degradation. Mol. Cell. Biol. 22, 626-634 (2002).

142. Tsai, Y. C. \& Weissman, A. M. The unfolded protein response, degradation from the endoplasmic reticulum, and cancer. Genes and Cancer 1, 764-778 (2010).

143. Travers, K. J. et al. Functional and genomic analyses reveal an essential coordination between the unfolded protein response and ER-associated degradation. Cell 101, 249258 (2000).

144. Rock, K. L. et al. Inhibitors of the proteasome block the degradation of most cell proteins and the generation of peptides presented on MHC class I molecules. Cell 78, 761-771 (1994).

145. Palombella, V. J., Rando, O. J., Goldberg, A. L. \& Maniatis, T. The ubiquitin-proteasome pathway is required for processing the NF-kappa B1 precursor protein and the activation of NF-kappa B. Cell 78, 773-785 (1994).

146. Adams, J. Proteasome inhibition in cancer: development of PS-341. Semin Oncol 28, 613-619 (2001).

147. Masdehors, P. et al. Increased sensitivity of CLL-derived lymphocytes to apoptotic death activation by the proteasome-specific inhibitor lactacystin. Br. J. Haematol. 105, 752-757 (1999).

148. Adams, J. et al. Potent and selective inhibitors of the proteasome: dipeptidyl boronic acids. Bioorg Med Chem Lett 8, 333-338 (1998).

149. An, B., Goldfarb, R. H., Siman, R. \& Ping Dou, Q. Novel dipeptidyl proteasome inhibitors overcome Bcl-2 protective function and selectively accumulate the cyclin-dependent kinase inhibitor p27 and induce apoptosis in transformed, but not normal, human fibroblasts. Cell Death Differ. 5, 1062-1075 (1998).

150. Goldberg, A. L. Development of proteasome inhibitors as research tools and cancer drugs. J. Cell Biol. 199, 583-588 (2012).

151. Goldberg, A. L. Probing the proteasome. Trends Cell Biol. 26, 792-794 (2016).

152. Kisselev, A. F., Callard, A. \& Goldberg, A. L. Importance of the different proteolytic sites 
of the proteasome and the efficacy of inhibitors varies with the protein substrate. $J$ Biol Chem 281, 8582-8590 (2006).

153. Screen, M. et al. Nature of pharmacophore influences active site specificity of proteasome inhibitors. J. Biol. Chem. 285, 40125-40134 (2010).

154. Kraus, M. et al. The novel $\beta 2$-selective proteasome inhibitor LU-102 synergizes with bortezomib and carfilzomib to overcome proteasome inhibitor resistance of myeloma cells. Haematologica 100, 1350-1360 (2015).

155. Groll, M. \& Huber, R. Substrate access and processing by the $20 \mathrm{~S}$ proteasome core particle. Int. J. Biochem. Cell Biol. 35, 606-616 (2003).

156. da Fonseca, P. C. \& Morris, E. P. Cryo-EM reveals the conformation of a substrate analogue in the human $20 \mathrm{~S}$ proteasome core. Nat. Commun. 6, 7573 (2015).

157. Morris, E. P. \& da Fonseca, P. C. A. High-resolution cryo-EM proteasome structures in drug development. Acta Crystallogr. D73, 522-533 (2017).

158. Unverdorben, P. et al. Deep classification of a large cryo-EM dataset defines the conformational landscape of the $26 \mathrm{~S}$ proteasome. Proc. Natl. Acad. Sci. 111, 5544-5549 (2014).

159. Wehmer, M. et al. Structural insights into the functional cycle of the ATPase module of the 26S proteasome. Proc. Natl. Acad. Sci. 114, 201621129 (2017).

160. Chen, S. et al. Structural basis for dynamic regulation of the human $26 \mathrm{~S}$ proteasome. Proc. Natl. Acad. Sci. 113, 12991-12996 (2016).

161. Guo, Q. et al. In situ structure of neuronal C9orf72 poly-GA aggregates reveals proteasome recruitment. Cell 172, 696-705 (2018).

162. Besche, H. C. \& Goldberg, A. L. Affinity purification of mammalian $26 \mathrm{~S}$ proteasomes using an ubiquitin-like domain. Methods Mol. Biol. 832, 423-432 (2012).

163. Kuo, C., Collins, G. A. \& Goldberg, A. L. Methods to rapidly prepare mammalian $26 \mathrm{~S}$ proteasomes for biochemical analysis. Methods Mol. Biol. 1844, 277-288 (2018).

164. Qiu, X. B. et al. hRpn13/ADRM1/GP110 is a novel proteasome subunit that binds the deubiquitinating enzyme, UCH37. EMBO J. 25, 5742-5753 (2006).

165. Husnjak, K. et al. Proteasome subunit Rpn13 is a novel ubiquitin receptor. Nature 453, 481-488 (2008).

166. Leggett, D. et al. Multiple associated proteins regulate proteasome structure and function. Mol Cell 10, 495-507 (2002).

167. Leggett, D. et al. Multiple associated proteins regulate proteasome structure and function. Mol. Cell 10, 495-507 (2002).

168. Guerrero, C., Tagwerker, C., Kaiser, P. \& Huang, L. An integrated mass spectrometrybased proteomic approach quantitative analysis of tandem affinity-purified in vivo crosslinked protein complexes (QTAX) to decipher the 26S proteasome-interacting network. Mol. Cell. Proteomics 5, 366-378 (2006).

169. Wang, X. et al. Molecular details underlying dynamic structures and regulation of the human 26 S proteasome. Mol. Cell. Proteomics 16, 840-854 (2017).

170. Wang, X. et al. Molecular details underlying dynamic structures and regulation of the human 26S proteasome. Mol. Cell. Proteomics (2017).

171. Choi, W. H. et al. Open-gate mutants of the mammalian proteasome show enhanced ubiquitin-conjugate degradation. Nat. Commun. 7, 10963 (2016).

172. Dechavanne, V., Vilbois, F., Glez, L. \& Antonsson, B. Purification and separation of the $20 \mathrm{~S}$ immunoproteasome from the constitutive proteasome and identification of the subunits by LC-MS. Protein Expr. Purif. 87, 100-110 (2013).

173. Roelofs, J., Suppahia, A., Waite, K. A. \& Park, S. Native gel approaches in studying proteasome assembly and chaperones. Methods Mol. Biol. 1844, 237-260 (2018).

174. Bhattacharyya, S., Renn, J. P., Yu, H., Marko, J. F. \& Matouschek, A. An assay for $26 \mathrm{~S}$ proteasome activity based on fluorescence anisotropy measurements of dye-labeled 
protein substrates. Anal. Biochem. 509, 50-59 (2016).

175. Thibaudeau, T. A., Anderson, R. T. \& Smith, D. M. A common mechanism of proteasome impairment by neurodegenerative disease-associated oligomers. Nat. Commun. 9, 1097 (2018).

176. Ghoda, L., Van Daalen Wetters, T., Macrae, M., Ascherman, D. \& Coffino, P. Prevention of rapid intracellular degradation of ODC by a carboxyl-terminal truncation. Science 243, 1493-1495 (1989).

177. Murakami, Y. et al. Ornithine decarboxylase is degraded by the $26 \mathrm{~S}$ proteasome without ubiquitination. Nature 360, 597-599 (1992).

178. Hoyt, M. A., Zhang, M. \& Coffino, P. Ubiquitin-independent mechanisms of mouse ornithine decarboxylase degradation are conserved between mammalian and fungal cells. J. Biol. Chem. 278, 12135-12143 (2003).

179. Henderson, A., Erales, J., Hoyt, M. A. \& Coffino, P. Dependence of proteasome processing rate on substrate unfolding. J. Biol. Chem. 286, 17495-17502 (2011).

180. Martinez-Fonts, K. \& Matouschek, A. A rapid and versatile method for generating proteins with defined ubiquitin chains. Biochemistry 55, 1898-1908 (2016).

181. Prakash, S., Tian, L., Ratliff, K. S., Lehotzky, R. E. \& Matouschek, A. An unstructured initiation site is required for efficient proteasome-mediated degradation. Nat Struct Mol Biol 11, 830-837 (2004).

182. Thrower, J. S., Hoffman, L., Rechsteiner, M. \& Pickart, C. M. Recognition of the polyubiquitin proteolytic signal. EMBO J. 19, 94-102 (2000).

183. Bence, N. F., Bennett, E. J. \& Kopito, R. R. Application and analysis of the GFPu family of ubiquitin-proteasome system reporters. Methods Enzymol. 399, 481-490 (2005).

184. Gilon, T., Chomsky, O. \& Kulka, R. G. Degradation signals for ubiquitin system proteolysis in Saccharomyces cerevisiae. EMBO J. 17, 2759-2766 (1998).

185. Bennett, E. J., Bence, N. F., Jayakumar, R. \& Kopito, R. R. Global impairment of the ubiquitin-proteasome system by nuclear or cytoplasmic protein aggregates precedes inclusion body formation. Mol. Cell 17, 351-365 (2005).

186. Wang, J. et al. Impaired ubiquitin-proteasome system activity in the synapses of Huntington's disease mice. J. Cell Biol. 180, 1177-1189 (2008).

187. Varshavsky, A., Bachmair, A. \& Finley, D. The N-end rule of selective protein turnover: mechanistic aspects and functional implications. Biochem Soc Trans 15, 815-816 (1987).

188. Dantuma, N. P., Lindsten, K., Glas, R., Jellne, M. \& Masucci, M. G. Short-lived green fluorescent proteins for quantifying ubiquitin/proteasome-dependent proteolysis in living cells. Nat. Biotechnol. 18, 538-543 (2000).

189. Varshavsky, A. Ubiquitin fusion technique and related methods. Methods Enzymol. 399, 777-799 (2005).

190. Dohmen, R. J. \& Varshavsky, A. Heat-inducible degron and the making of conditional mutants. Methods Enzymol. 399, 799-822 (2005).

191. DeLaBarre, B., Christianson, J. C., Kopito, R. R. \& Brunger, A. T. Central pore residues mediate the p97/VCP activity required for ERAD. Mol Cell 22, 451-462 (2006).

192. Otero, M. G. et al. Fast axonal transport of the proteasome complex depends on membrane interaction and molecular motor function. J. Cell Sci. 127, 1537-1549 (2014).

193. Reits, E. et al. Peptide diffusion, protection, and degradation in nuclear and cytoplasmic compartments before antigen presentation by MHC class I molecules. Immunity 18, 97108 (2003).

194. Groothuis, T. A. \& Reits, E. A. Monitoring the distribution and dynamics of proteasomes in living cells. Methods Enzymol. 399, 549-563 (2005).

195. Luker, G. D., Pica, C. M., Song, J., Luker, K. E. \& Piwnica-Worms, D. Imaging 26 S proteasome activity and inhibition in living mice. Nat. Med. 9, 969-973 (2003).

196. Lindsten, K., Menendez-Benito, V., Masucci, M. G. \& Dantuma, N. P. A transgenic mouse 
model of the ubiquitin/proteasome system. Nat Biotechnol 21, 897-902 (2003).

197. Menéndez-Benito, V., Heessen, S. \& Dantuma, N. P. Monitoring of ubiquitin-dependent proteolysis with green fluorescent protein substrates. Methods Enzymol. 399, 490-511 (2005).

198. Hamer, G., Matilainen, O. \& Holmberg, C. I. A photoconvertible reporter of the ubiquitinproteasome system in vivo. Nat. Methods 7, 473-478 (2010).

199. Hewings, D. S., Flygare, J. A., Wertz, I. E. \& Bogyo, M. Activity-based probes for the multicatalytic proteasome. FEBS J. 284, 1540-1554 (2017).

200. Hideshima, T. et al. NF-kappa B as a therapeutic target in multiple myeloma. J Biol Chem 277, 16639-16647 (2002).

201. Adams, J. Potential for proteasome inhibition in the treatment of cancer. Drug Discov Today 8, 307-315 (2003).

202. Chhabra, S. Novel proteasome inhibitors and histone deacetylase inhibitors: progress in myeloma therapeutics. Pharmaceuticals 10, E40 (2017).

203. Chen, X., Barton, L. F., Chi, Y., Clurman, B. E. \& Roberts, J. M. Ubiquitin-independent degradation of cell-cycle inhibitors by the REGy proteasome. Mol. Cell 26, 843-852 (2007).

204. Lü, S. et al. Overexpression of the PSMB5 gene contributes to bortezomib resistance in T-lymphoblastic lymphoma/leukemia cells derived from Jurkat line. Exp. Hematol. 36, 1278-1284 (2008).

205. Oerlemans, R. et al. Molecular basis of bortezomib resistance: Proteasome subunit $\beta 5$ (PSMB5) gene mutation and overexpression of PSMB5 protein. Blood 112, 2489-2499 (2008).

206. Zhu, Y. X. et al. RNAi screen of the druggable genome identifies modulators of proteasome inhibitor sensitivity in myeloma including CDK5. Blood 117, 3847-3857 (2011).

207. Smith, A. J. et al. Noxa/Bcl-2 protein interactions contribute to bortezomib resistance in human lymphoid cells. J. Biol. Chem. 286, 17682-17692 (2011).

208. Hideshima, T. et al. Molecular characterization of PS-341 (bortezomib) resistance: Implications for overcoming resistance using lysophosphatidic acid acyltransferase (LPAAT)-beta inhibitors. Oncogene 24, 3121-3129 (2005).

209. Hao, M. et al. Bone marrow stromal cells protect myeloma cells from bortezomib induced apoptosis by suppressing microRNA-15a expression. Leuk. Lymphoma 52, 1787-1794 (2011).

210. Kerckhove, N. et al. Long-term effects, pathophysiological mechanisms, and risk factors of chemotherapy-induced peripheral neuropathies: A comprehensive literature review. Front. Pharmacol. 8, 86 (2017).

211. Schlafer, D., Shah, K. S., Panjic, E. H. \& Lonial, S. Safety of proteasome inhibitors for treatment of multiple myeloma. Expert Opin Drug Saf. 16, 167-83 (2017).

212. Carozzi, V. A. et al. Neurophysiological and neuropathological characterization of new murine models of chemotherapy-induced chronic peripheral neuropathies. Exp. Neurol. 226, 301-309 (2010).

213. Staff, N. P. et al. Bortezomib alters microtubule polymerization and axonal transport in rat dorsal root ganglion neurons. Neurotoxicology 39, 124-131 (2013).

214. Dasuri, K. et al. Selective vulnerability of neurons to acute toxicity after proteasome inhibitor treatment: implications for oxidative stress and insolubility of newly synthesized proteins. Free Radic. Biol. Med. 49, 1290-1297 (2010).

215. Arastu-Kapur, S. et al. Nonproteasomal targets of the proteasome inhibitors bortezomib and carfilzomib: a link to clinical adverse events. Clin. Cancer Res. 17, 2734-2743 (2011).

216. Bachovchin, D. A. et al. Superfamily-wide profiling of enzyme activity. Nat. Chem. Biol. 
10, 656-663 (2014).

217. Csizmadia, V. et al. Proteasome inhibitors bortezomib and carfilzomib used for the treatment of multiple myeloma do not inhibit the serine protease HtrA2/Omi. Toxicol. Res. 5, 1619-1628 (2016).

218. Adams, J. Proteasome inhibitors as new anticancer drugs. Curr Opin Oncol 14, 628-634 (2002).

219. Steele, J. M. Carfilzomib: A new proteasome inhibitor for relapsed or refractory multiple myeloma. J. Oncol. Pharm. Pract. 19, 348-354 (2013).

220. Sugumar, D., Keller, J. \& Vij, R. Targeted treatments for multiple myeloma: Specific role of carfilzomib. Pharmgenomics. Pers. Med. 8, 23-33 (2015).

221. Dimopoulos, M. A. et al. Carfilzomib or bortezomib in relapsed or refractory multiple myeloma (ENDEAVOR): An interim overall survival analysis of an open-label, randomised, phase 3 trial. Lancet Oncol. 18, 1327-1337 (2017).

222. Buac, D. et al. From bortezomib to other inhibitors of the proteasome and beyond. Curr. Pharm. Des. 19, 4025-38 (2013).

223. Ao, L. et al. Development of peptide-based reversing agents for P-glycoprotein-mediated resistance to carfilzomib. Mol. Pharm. 9, 2197-2205 (2012).

224. Fan, Q. \& Liu, B. Identification of the anticancer effects of a novel proteasome inhibitor, ixazomib, on colorectal cancer using a combined method of microarray and bioinformatics analysis. Onco. Targets. Ther. 10, 3591-3606 (2017).

225. $\mathrm{Li}, \mathrm{H}$. et al. Novel proteasome inhibitor ixazomib sensitizes neuroblastoma cells to doxorubicin treatment. Sci. Rep. 6, 34397 (2016).

226. Esparza-López, J. et al. Doxorubicin induces atypical NF-KB activation through c-Abl kinase activity in breast cancer cells. J. Cancer Res. Clin. Oncol. 139, 1625-1635 (2013).

227. Enzler, T. et al. Cell-selective inhibition of NF-KB signaling improves therapeutic index in a melanoma chemotherapy model. Cancer Discov. 1, 496-507 (2011).

228. Moreau, P. et al. Oral ixazomib, lenalidomide, and dexamethasone for multiple myeloma. N. Engl. J. Med. 374, 1621-1634 (2016).

229. Muz, B. et al. Spotlight on ixazomib: Potential in the treatment of multiple myeloma. Drug Des. Devel. Ther. 10, 217-226 (2016).

230. Mateos, M.-V. et al. Impact of prior therapy on the efficacy and safety of oral ixazomiblenalidomide-dexamethasone vs placebo-lenalidomide-dexamethasone in patients with relapsed/refractory multiple myeloma in TOURMALINE-MM1. Haematologica 102, 17671775 (2017).

231. Dou, Q. P. \& Zonder, J. A. Overview of proteasome inhibitor-based anti-cancer therapies: Perspective on bortezomib and second generation proteasome inhibitors versus future generation inhibitors of ubiquitin-proteasome system. Curr. Cancer Drug Targets 14, 517-536 (2014).

232. Chauhan, D. et al. A novel orally active proteasome inhibitor induces apoptosis in multiple myeloma cells with mechanisms distinct from Bortezomib. Cancer Cell 8, 407-419 (2005).

233. Levin, N. et al. Marizomib irreversibly inhibits proteasome to overcome compensatory hyperactivation in multiple myeloma and solid tumour patients. Br. J. Haematol. 174, 711-720 (2016).

234. Di, K. et al. Marizomib activity as a single agent in malignant gliomas: Ability to cross the blood-brain barrier. Neuro. Oncol. 18, 840-848 (2016).

235. Van Tellingen, $O$. et al. Overcoming the blood-brain tumor barrier for effective glioblastoma treatment. Drug Resist. Updat. 19, 1-12 (2015).

236. Laubach, J. P. et al. Clinical challenges associated with bortezomib therapy in multiple myeloma and Waldenstroms Macroglobulinemia. Leuk Lymphoma 50, 694-702 (2009).

237. Roccaro, A. et al. Selective inhibition of chymotrypsin-like activity of the 
immunoproteasome and constitutive proteasome in Waldenström macroglobulinemia. Blood 115, 4051-4060 (2010).

238. J. Kuhn, D., Z. Orlowski, R. \& C. Bjorklund, C. Second generation proteasome inhibitors: carfilzomib and immunoproteasome-specific inhibitors (IPSIs). Curr. Cancer Drug Targets 11, 285-295 (2011).

239. Basler, M., Mundt, S., Bitzer, A., Schmidt, C. \& Groettrup, M. The immunoproteasome: a novel drug target for autoimmune diseases. Clin. Exp. Rheumatol. 33, 74-79 (2015).

240. Liu, H. et al. PR-957, a selective inhibitor of immunoproteasome subunit low-MW polypeptide 7, attenuates experimental autoimmune neuritis by suppressing Th17-cell differentiation and regulating cytokine production. FASEB J. 31, 1756-1766 (2017).

241. Muchamuel, T. et al. A selective inhibitor of the immunoproteasome subunit LMP7 blocks cytokine production and attenuates progression of experimental arthritis. Nat. Med. 15, 781-787 (2009).

242. Ichikawa, H. T. et al. Beneficial effect of novel proteasome inhibitors in murine lupus via dual inhibition of type i interferon and autoantibody-secreting cells. Arthritis Rheum. 64, 493-503 (2012).

243. von Brzezinski, L. et al. Low neurotoxicity of ONX-0914 supports the idea of specific immunoproteasome inhibition as a side-effect-limiting, therapeutic strategy. Eur. J. Microbiol. Immunol. 7, 234-245 (2017).

244. Johnson, H. W. B. et al. Discovery of highly selective inhibitors of the immunoproteasome low molecular mass polypeptide 2 (LMP2) subunit. ACS Med. Chem. Lett. 8, 413-417 (2017).

245. Holbeck, S. et al. The National Cancer Institute ALMANAC: A Comprehensive Screening Resource for the Detection of Anticancer Drug Pairs with Enhanced Therapeutic Activity. Cancer Res 77, 3564-3576 (2017).

246. Kisselev, A. F., van der Linder, W. \& Overkleeft, H. S. Proteasome inhibitors: An expanding army attacking a unique target. Chem Biol 19, 99-115 (2012).

247. Verma, R. et al. Role of Rpn11 metalloprotease in deubiquitination and degradation by the 26S proteasome. Science 298, 611-615 (2002).

248. Li, J. et al. Capzimin is a potent and specific inhibitor of proteasome isopeptidase Rpn11. Nat. Chem. Biol. 13, 486-493 (2017).

249. Borodovsky, A. et al. A novel active site-directed probe specific for deubiquitylating enzymes reveals proteasome association of USP14. EMBO J. 20,5187-5196 (2001).

250. Lee, B.-H. H. et al. Enhancement of proteasome activity by a small-molecule inhibitor of USP14. Nature 467, 179-184 (2010).

251. Kim, H. T. \& Goldberg, A. L. The deubiquitinating enzyme Usp14 allosterically inhibits multiple proteasomal activities and ubiquitin-independent proteolysis. J. Biol. Chem. 292, 9830-9839 (2017).

252. Boselli, M. et al. An inhibitor of the proteasomal deubiquitinating enzyme USP14 induces tau elimination in cultured neurons. J. Biol. Chem. 292, 19209-19225 (2017).

253. Haass, C. \& Selkoe, D. J. Soluble protein oligomers in neurodegeneration: Lessons from the Alzheimer's amyloid $\beta$-peptide. Nat. Rev. Mol. Cell Biol. 8, 101-112 (2007).

254. Castillo-Carranza, D. L., Guerrero-Muñoz, M. J. \& Kayed, R. Therapeutic approaches against common structural features of toxic oligomers shared by multiple amyloidogenic proteins. Biochem. Pharmacol. 88, 468-478 (2014).

255. Bence, N. F., Sampat, R. M. \& Kopito, R. R. Impairment of the ubiquitin-proteasome system by protein aggregation. Science 292, 1552-1555 (2001).

256. Tseng, B. P., Green, K. N., Chan, J. L., Blurton-jones, M. \& Laferla, F. M. Abeta inhibits the proteasome and enhances amyloid and tau accumulation. Neurobiol. Aging 29, 1607-1618 (2008).

257. Deriziotis, P. et al. Misfolded PrP impairs the UPS by interaction with the $20 \mathrm{~S}$ proteasome 
and inhibition of substrate entry. EMBO J. 30, 3065-3077 (2011).

258. Díaz-Hernández, M. et al. Inhibition of 26 S proteasome activity by huntingtin filaments but not inclusion bodies isolated from mouse and human brain. J. Neurochem. 98, 15851596 (2006).

259. Cecarini, V. et al. Amyloid peptides in different assembly states and related effects on isolated and cellular proteasomes. Brain Res. 1209, 8-18 (2008).

260. Lindersson, E. et al. Proteasomal inhibition by $\alpha$-synuclein filaments and oligomers. J. Biol. Chem. 279, 12924-12934 (2004).

261. Kayed, R. et al. Common structure of soluble amyloid oligomers implies common mechanism of pathogenesis. Science 300, 486-489 (2003). 


\title{
Study 1: A common mechanism of proteasome impairment by neurodegenerative disease-associated oligomers
}

\author{
Tiffany A. Thibaudeau ${ }^{1}$, Raymond T. Anderson ${ }^{2}$, and David M. Smith ${ }^{1,2^{*}}$ \\ Nature Communications, volume 9, Article number: 1097 (2018) \\ DOI: https://doi.org/10.1038/s41467-018-03509-0
}

\begin{abstract}
${ }^{1}$ Program in Biochemistry and Molecular Biology, Department of Biochemistry, West Virginia University, Morgantown, WV

${ }^{2}$ Department of Neuroscience, West Virginia University, Morgantown, WV

* Corresponding Author: David M. Smith, West Virginia University Department of Biochemistry, P.O. Box 9142, Morgantown WV, 26506. Email: dmsmith@hsc.wvu.edu
\end{abstract}

Keywords: Amyloid- $\beta$, proteasome, oligomers

Financial support: NIH-R01GM107129 to D.M.S.

The content is solely the responsibility of the authors and does not necessarily represent the official views of the $\mathrm{NIH}$.

This article is licensed under a Creative Commons Attribution 4.0 International License: https://creativecommons.org/licenses/by/4.0/legalcode. Modified with minor text edits and addition of statistical analyses to figures. 


\section{Abstract}

Protein accumulation and aggregation with a concomitant loss of proteostasis contributes to the vast majority of neurodegenerative diseases. Because the ubiquitin-proteasome system (UPS) plays a major role in protein degradation and proteostasis we hypothesized that a general mechanism of UPS impairment could contribute to neurodegenerative diseases. Here, we show that three different proteins from Alzheimer's, Parkinson's, and Huntington's disease that misfold and oligomerize into a shared three-dimensional structure potently impair the proteasome. This study indicates that the shared conformation allows these oligomers to bind to and inhibit the proteasome with low nM affinity, impairing ubiquitin-dependent and ubiquitin-independent proteasome function in brain lysates. Detailed mechanistic analysis demonstrates that these oligomers inhibit the $20 S$ proteasome through allosteric impairment of the substrate-gate in the $20 \mathrm{~S}$ core particle, preventing the $19 \mathrm{~S}$ regulatory particle from injecting substrates into the degradation chamber. These results provide a novel molecular model for oligomer-driven impairment of proteasome function that is relevant to a variety of neurodegenerative diseases, irrespective of the specific misfolded protein that is involved. 


\section{Introduction}

The most common neurodegenerative diseases are characterized by an accumulation of aggregation-prone proteins concomitant with a loss of proteostasis, that results in progressive death of neurons ${ }^{1-3}$. Culminating evidence from the past two decades has revealed that soluble, oligomeric forms of protein aggregates (such as $A \beta$ in Alzheimer's disease, $\alpha$-Synuclein in Parkinson's disease, and mutant huntingtin in Huntington's disease) are likely the most toxic species ${ }^{4,5}$. While different regions of the brain are affected in these distinct diseases, proteotoxicity is a shared feature found in these affected regions of the brain. This suggests that a common mechanism of proteotoxicity could contribute to the development and progression of these distinct neurodegenerative diseases.

Proteostasis ${ }^{6,7}$ is maintained by several systems in the cell including the ubiquitin-proteasome system (UPS), chaperones, chaperone-mediated autophagy, and macroautophagy ${ }^{8}$. The ubiquitin proteasome system (UPS) is the principle route for the degradation of intracellular misfolded, damaged, or unneeded proteins ${ }^{9}$. If the efficiency of proteostasis systems declines, misfolded proteins accumulate and aggregate in the cell, which can disrupt normal cellular functions and even cause cell death ${ }^{10}$. Maintaining proteostasis is especially important for neurons due to their complex architecture, long lifespan, and inability to dilute aggregate load by cell division ${ }^{11}$. Most importantly, the UPS is critical for normal functioning of neuronal synapses, including synaptic protein turnover, plasticity, and long-term memory formation, which rely on tightly controlled changes in the proteome ${ }^{11-15}$. Recently, Ramachandran \& Margolis (2017) ${ }^{16}$ identified a mammalian nervous-system-specific membrane proteasome complex that directly and rapidly modulates neuronal function by degrading intracellular proteins into extracellular peptides that stimulate neuronal signaling through postsynaptic $\mathrm{N}$-methyl-D-aspartate receptors. 
Decreased proteasome function has been reported in a broad array of chronic neurodegenerative diseases ${ }^{17}$. Impaired proteasome function has been implicated, as a primary cause or a secondary consequence, in the in the pathogenesis of many neurodegenerative diseases, including Alzheimer's, Parkinson's, and Huntington's diseases ${ }^{2,17-21}$. In fact, brain region-specific proteasome inhibition (e.g. forebrain, substantia nigra) closely mirrors the neuropathology and clinical hallmarks of neurodegenerative diseases ${ }^{22-26}$. A small percentage of neurodegenerative disease are caused by hereditary gene mutations, many of which affect components of the UPS (e.g. PARK1, PINK $)^{20}$. However, the vast majority of neurodegeneration is idiopathic in origin and the involvement of the UPS is less clear ${ }^{17}$. What is clear in these diseases is that proteins that are normally degraded are not properly degraded after misfolding occurs, leading to their accumulation. Several groups have provided evidence that aggregated proteins from neurodegenerative diseases interact with and impair proteasome function ${ }^{27-37}$. However, it is not clear what specific types of aggregates impair the proteasome, and a mechanistic understanding of how they do so has not been elucidated. One study has been able to show that heterogeneous aggregates of the mouse prion protein, PrPsc, reduced substrate entry by decreasing proteasomal gating ${ }^{36}$. Despite these many efforts, an understanding of why and how the proteasome is so generally impaired in neurodegenerative disease has remained elusive. Understanding the mechanism of impairment will provide a basis for drug development to restore proteasome activity and proteostasis in the brain and is therefore an important effort.

Proteins targeted for proteasomal degradation are marked by the attachment of several ubiquitin proteins. These poly-ubiquitinated substrates are recognized by the $26 \mathrm{~S}$ proteasome and are degraded $^{9}$. The $26 \mathrm{~S}$ proteasome is made up of a $20 \mathrm{~S}$ proteasome core particle capped on one or both ends by the $19 \mathrm{~S}$ regulatory particle. It degrades proteins by a multistep process: the $19 \mathrm{~S}$ regulatory particle binds ubiquitinated substrates and opens a substrate entry gate in the $20 S^{38-}$ ${ }^{40}$ and unfolds its substrates by translocating them into the $20 \mathrm{~S}$ catalytic chamber where they are 
degraded ${ }^{41,42}$. The $20 S$ is a hollow cylindrical complex composed of four heteroheptameric rings arranged in a $\alpha_{7}-\beta_{7-}-\beta_{7}-\alpha_{7}$ fashion ${ }^{43}$. Proteolysis occurs on the interior surface of $\beta$-subunit rings. The substrate gate is formed by the $\mathrm{N}$-termini of the $\alpha$-subunits, which prevent unregulated access to the catalytic sites by folding over the entry pore and blocking substrate translocation into the catalytic chamber ${ }^{44}$. Triggering of gate-opening by the $19 \mathrm{~S}$ requires the C-terminal HbYX motif of the 19S ATPases to bind to intersubunit pockets (between the $\alpha$-subunits) on top of the $20 \mathrm{~S}^{45}$. The HbYX motif allows the 19S to bind to the 20S core particle, but binding of the HbYX motif by itself (as a hepta-peptide) is also sufficient to allosterically induce conformational changes in the $\alpha$-subunits that cause gate opening ${ }^{45-48}$. Clearly, regulation of the $20 S$ proteasome gate is an important aspect of proteasome function and the cell has evolved many different proteasomal regulators that control $20 \mathrm{~S}$ gate-opening, many of which contain the HbYX motif (e.g. the 19S ATPases: Rpt2, Rpt3, Rpt5; Blm10/PA200; Pba1-Pba2; PI31; and archaeal CDC48/P97), and some that do not (i.e. the 11 family: PA28 $\alpha \beta$ and PA26) $)^{48}$.

This study demonstrates that misfolded proteins from three distinct neurodegenerative diseases adopt a common three-dimensional conformation that is capable of impairing ubiquitin-dependent and ubiquitin-independent proteasome function. Although these oligomers possess unique primary sequences, they all impair the proteasome through allosteric stabilization of the closed gated conformation of the $20 \mathrm{~S}$ core particle, therein blocking protein degradation. Moreover, these toxic oligomers specifically impair HbYX motif dependent gate-opening, yet do not impair gate-opening induced by the $11 \mathrm{~S}$ family of regulators. These data suggest that proteasome impairment in various neurodegenerative diseases may share a common mechanism.

\section{Results}

Prior studies report conflicting observations regarding the impairment of the proteasome by disease-related aggregated proteins, some demonstrating proteasome impairment ${ }^{27-30,32-35}$ while others do not ${ }^{49,50}$. The major limitation of these studies is that the conformational state of the 
aggregates was not accounted for or considered. Aggregation-prone proteins have the unique property of conformational polymorphism. During amyloid formation a variety of aggregate species are formed, ranging from small dimers up to large insoluble fibrils. Oligomers are metastable intermediates to fibril formation or an off-pathway product of aggregation and are recognized as the primary pathogenic effectors ${ }^{51}$. Since the previous studies used heterogeneous compositions of the aggregated proteins, these seemingly conflicting results for proteasome impairment are not surprising. In this study, we purify to homogeneity a specific conformation of a pathological oligomer, identified its conformational status, and extensively characterize its mechanism of impairment on human and mammalian 20S/26S proteasomes. In addition, this study identifies a specific oligomeric conformation found in Alzheimer's, Parkinson's, and Huntington's disease that substantially impairs proteasome function in a way that could contribute to the development and progression of these and other neurodegenerative diseases.

To determine if specific types of oligomers are responsible for proteasome impairment we began by generating various mixed populations of protein aggregates made from either amyloid- $\beta$ 1-42 $(A \beta), \alpha$-synuclein $(\alpha-S y n)$, or huntingtin exon 1 with a polyQ-expansion (Htt-53Q) and asked if they could impair purified mammalian $20 \mathrm{~S}$ proteasome. We found that under specific oligomerization conditions (different for each protein type) each of the aggregate preparations could significantly impair the 20 S proteasomes ability to hydrolyze fluorogenic peptide substrates (Fig. 1A). These results replicate those which have been reported to some extent previously ${ }^{29-31}$. Next, we separated the mixed aggregates into soluble and insoluble fractions and again tested their effect on proteasome activity. The soluble oligomers, but not equal amounts of monomers or insoluble fibrils, strongly impaired proteasome activity (Fig. 1B) in a concentration-dependent manner (Supplementary Fig. 1). The eukaryotic proteasome has three types of active sites, each displaying preference for cleavage after specific residues (chymotrypsin-like, hydrophobic; caspase-like, 
acidic; trypsin-like, basic). Substrate hydrolysis by all three of the catalytic sites were impaired by the soluble oligomers (Supplementary Fig. 2).
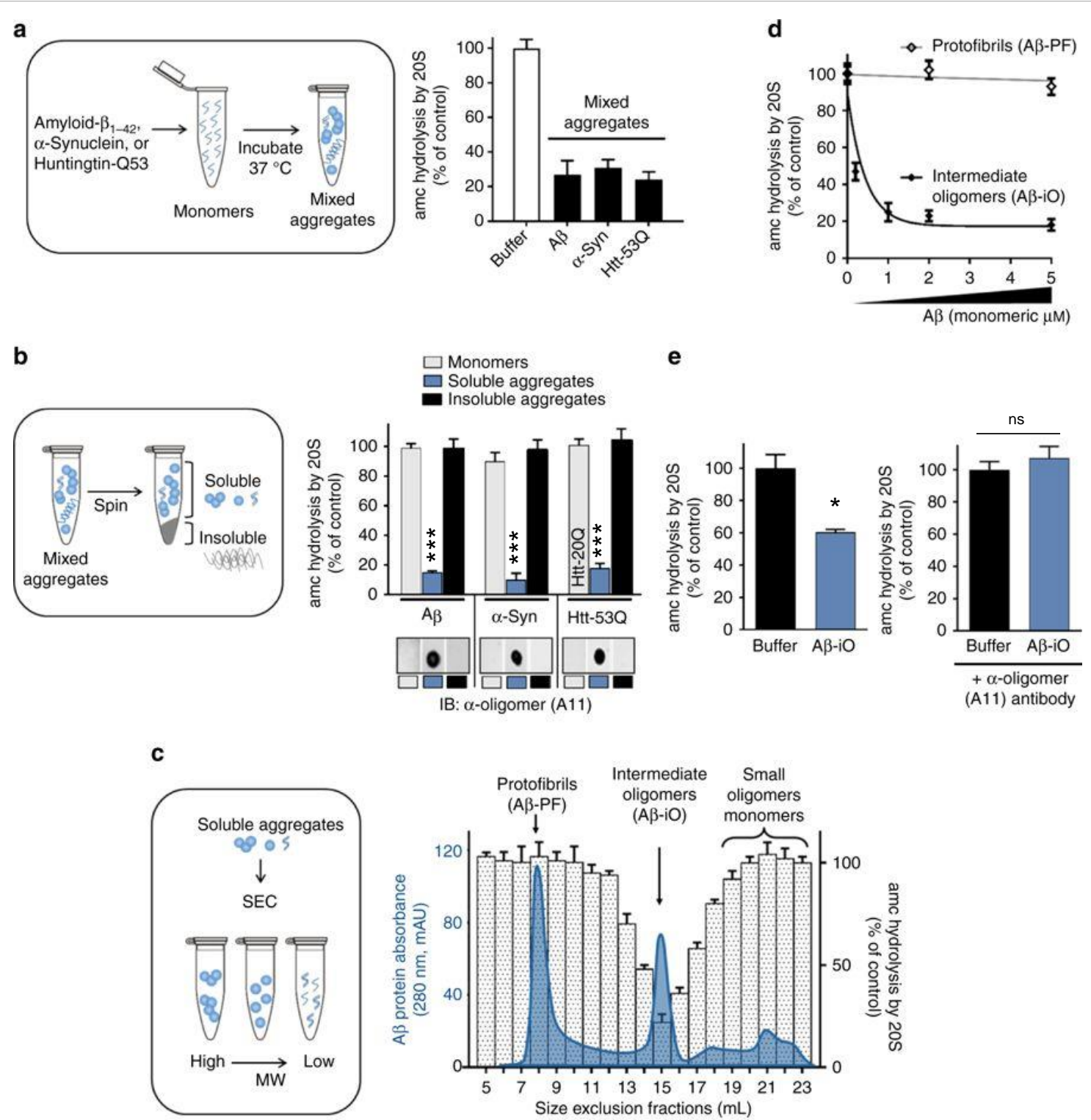

Dot blot:

Size exclusion fractions $(\mathrm{mL})$

Figure 1. A specific conformation of soluble oligomers potently inhibits the mammalian 20S proteasome. (a) Mammalian $20 \mathrm{~S}$ proteasomes were incubated with mixed aggregates of $\mathrm{A} \beta 1-42(5 \mu \mathrm{M}), \alpha-\operatorname{Syn}(1 \mu \mathrm{M}), \mathrm{Htt}-53 \mathrm{Q}(0.1 \mu \mathrm{M})$, or an equal volume of oligomer buffer (control). Proteasome activity (linear rate of LLVY-amc hydrolysis) is represented as a percentage of activity compared to the control. (b) Crude aggregates from $A$ were separated into soluble and insoluble aggregates (schematic, left) and were assayed as in A (bar graph, right). For huntingtin monomers, Htt-20Q monomers were used because pure Htt-53Q monomers could not be obtained due to rapid oligomerization. Dot blots of monomers, soluble aggregates, and insoluble aggregates from $B$ were probed with the conformation-dependent anti-oligomer ' $A 11$ ' antibody (bottom right). (c) Soluble $A \beta$ aggregates from $B$ were separated by size exclusion chromatography (SEC) (Abs $280 \mathrm{~nm}$, solid blue line). $2 \mu$ from each fraction was evaluated for its effect on $20 \mathrm{~S}$ proteasome chymotrypsin-like activity (bars) and probed for anti-oligomer A11 reactivity (dot blot, bottom). (d) Proteasome activity with up to $5 \mu \mathrm{M}$ of $A \beta$ oligomers $(A \beta-i O)$ or $A \beta$ protofibrils $(A \beta-P F)$ from $C$. (e) Intermediate oligomers from $D$ were pre-incubated with anti-oligomer $A 11$ antibody $(A \beta-i O+A 11)$ or an equal volume of antibody buffer $(A \beta-i O)$ for 30 minutes at $37^{\circ} \mathrm{C}$ before to addition to proteasome activity assay. Final concentration of $A \beta-i O$ in the assay was $0.25 \mu M$ (the $\sim \mathrm{IC} 50$ as determined in $D)$. The concentrations of aggregates are calculated based on the respective monomeric peptide/protein mass $(A \beta$, $4.5 \mathrm{kDa} ; \alpha-S y n, 14 \mathrm{kDa}$; and Htt-53Q, $22 \mathrm{kDa}$ ). All controls contained an equal volume of buffer identical to that of the respective aggregates. Data are representative of three or more independent experiments performed in triplicate. Error bars represent \pm standard deviation. ${ }^{* * *} p<0.0001 ;{ }^{*} p<0.05 ;$ ns $p>0.05$ (students $t$ test) 
a

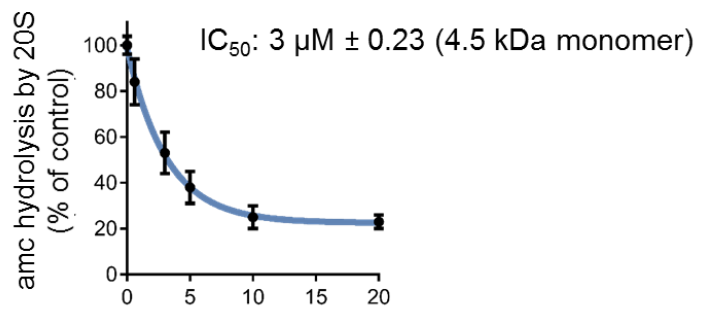

$\mathrm{A} \beta \mathrm{A} 11(+)$ mixed aggregates

(monomeric $\mu \mathrm{M})$

C

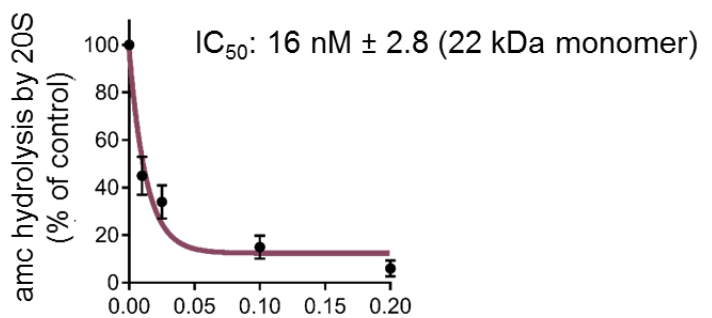

Htt-53Q A11(+) oligomers (monomeric $\mu \mathrm{M}$ ) b

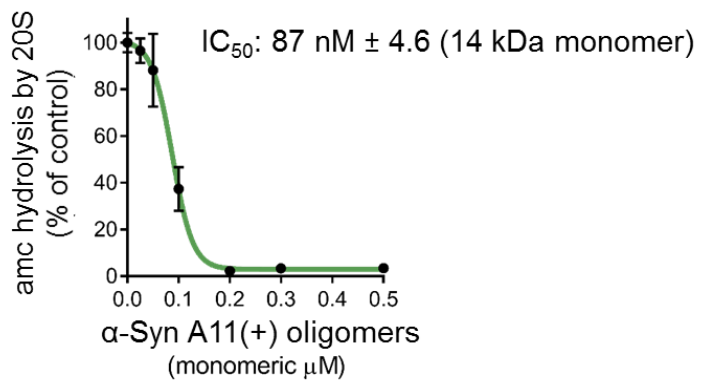

Supplemental Figure 1. Corresponds to Figure $1 B$.

A11(+) soluble aggregates of $A \beta, \alpha-S y n$, and $H t t-53 Q$ impair the mammalian $20 \mathrm{~S}$ proteasome in a concentration-dependent manner. Mammalian 20S proteasomes were incubated with soluble aggregates of $A \beta(\boldsymbol{a}), \alpha$-Syn $(\boldsymbol{b})$, or $\mathrm{Htt}-53 \mathrm{Q}(\boldsymbol{c})$ at the indicated concentrations and proteasome activity (LLVY-amc hydrolysis) was measured. $A \beta$ mixed aggregates are the same as used in Figure 1a. Soluble oligomers from $\alpha-S y n$ and $H \mathrm{Ht}-53 \mathrm{Q}$ are from Figure $1 \mathrm{~b}$. Half-maximal inhibition of the $20 \mathrm{~S}$ is indicated. The concentrations of aggregates are calculated based on the respective monomeric peptide/protein mass (A $\beta, 4.5 \mathrm{kDa}$; $\alpha$-Syn, $14 \mathrm{kDa}$; and $\mathrm{Htt}-53 \mathrm{Q}, 22$ $\mathrm{kDa})$. All controls contained an equal volume of buffer identical to that of the respective aggregates. Data is representative of three independent experiments performed in triplicate. Error bars represent \pm standard deviation. Sigmoidal dose response (variable slope) line fit performed with GraphPad version 7.

a

Chymotrypsin-like Activity

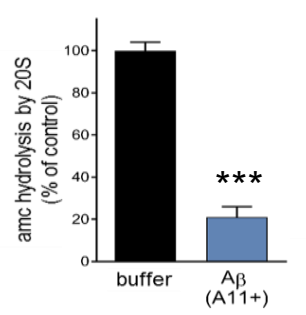

b

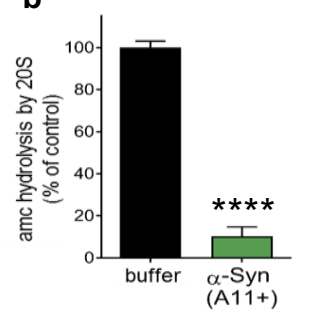

C

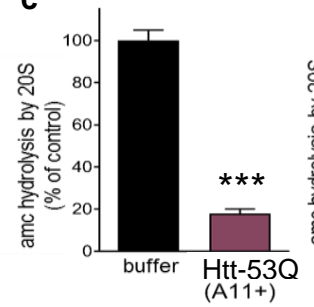

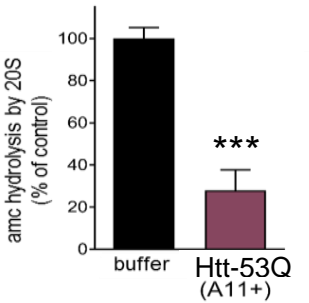

Trypsin-like Activity
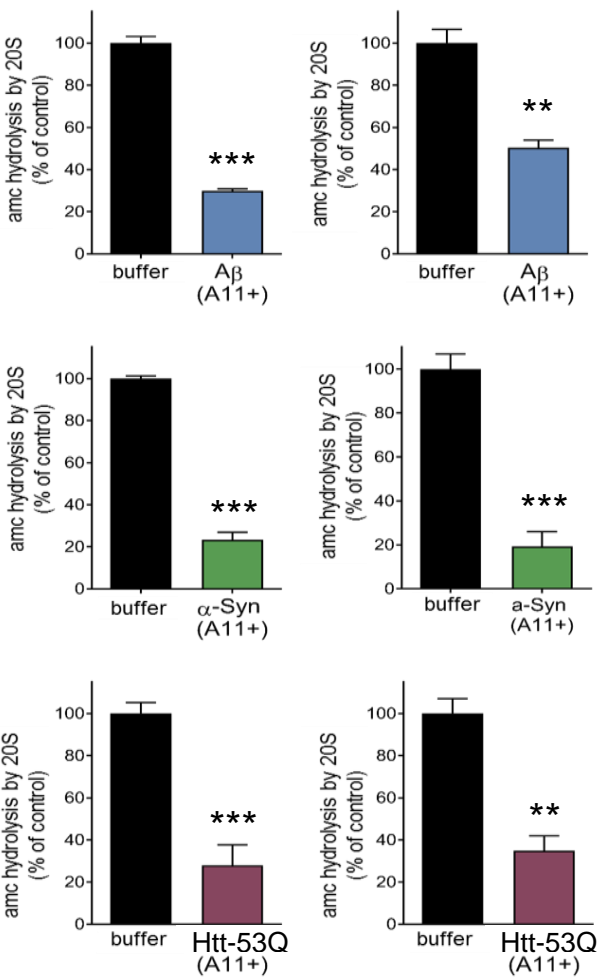

Supplemental Figure 2. Corresponds to Figure $1 B$.

A11(+) oligomers from $A \beta, \alpha-S y n$, and $\mathrm{Htt}-53 Q$ impair substrates that are specific for all three proteolytic sites in the mammalian 20S proteasome. Mammalian 20S proteasomes were incubated with $\mathrm{A} \beta$ oligomers $(2 \mu \mathrm{M})(\boldsymbol{a}), \alpha$-Syn $\mathrm{A} 11+$ oligomers $(1 \mu \mathrm{M})(\boldsymbol{b})$, or Htt-53Q A $11+$ oligomers $(0.1 \mu \mathrm{M})(\boldsymbol{c})$, and proteasome chymotrypsin-like (LLV Y-amclysisolysis), caspase-like (nLP ysis) activity was measured. Chymotrypsin-like and caspase-like activity assays used $0.5 \mathrm{nM}$ of $20 \mathrm{~S}$ proteasome, trypsin-like activity assays used $1 \mathrm{nM}$ of $20 \mathrm{~S}$ proteasome. The concentrations of aggregates are calculated based on the respective monomeric peptide/protein mass (A $\beta, 4.5 \mathrm{kDa} ; \alpha$-Syn, $14 \mathrm{kDa}$; and $\mathrm{Htt}-53 \mathrm{Q}, 22 \mathrm{kDa}$ ). All controls contained an equal volume of buffer identical to that of the respective aggregates. Data is representative of three independent experiments performed in triplicate. Error bars represent \pm standard deviation. ${ }^{* * * *} p<0.0001 ;{ }^{* * *} p<0.001 ;{ }^{* *} p<0.01$ (students $t$ test). 
$\mathrm{A} \beta, \alpha-\mathrm{Syn}$, and $\mathrm{Htt}-53 \mathrm{Q}$ monomers are relatively unstructured and they can enter the $20 \mathrm{~S}$ proteasome to be degraded. However, the monomers fail to impair peptide hydrolysis by the proteasome at equal concentrations as the oligomers (Fig. 1B) therefore, substrate competition at the active site cannot explain impairment by the oligomers. Furthermore, oligomers are too large (Fig. 2G-H) to enter the $13 \AA$ wide substrate-entry channel of the 20 S proteasome. Additionally, since the insoluble aggregates of these proteins cannot impair the proteasome (Fig. 1B), this suggests that impairment by the oligomers may be due to a specific conformation of the soluble oligomers which is lost after conversion to larger aggregates or fibrils. This is consistent with literature that ascribes cellular toxicity to soluble oligomers in neurodegenerative diseases ${ }^{4,52,53}$. Many species of oligomeric structures have been described, and antibodies developed to recognize specific structural conformations of disease related species ${ }^{54-56}$. Kayed et al. $(2003)^{55}$ generated a polyclonal anti-oligomer antibody (A11) that specifically recognizes some types of protein oligomers independent of the proteins amino acid sequence. This A11 antibody recognizes some oligomeric species of $A \beta$, polyglutamine proteins, $\alpha$-synuclein, and prion, and has been used to assess the presence of oligomers in diseased brains compared to aged matched controls ${ }^{55,57}$. We performed a dot blot with A11 on the monomers, oligomers, and insoluble fibrils for each protein that we tested. All three of the soluble oligomer preparations contained the A11 epitope (A11+), while the epitope was absent in the monomeric and fibril fractions (Fig. 1B bottom).

It is interesting that all three soluble oligomer types that impaired proteasome activity also showed strong A11 antibody binding. To correlate proteasome impairment with the presence of the A11 epitope more specifically, the soluble fraction of the $A \beta$ aggregates were separated by size exclusion chromatography. Three prominent populations of soluble aggregates were observed, one in the void volume consistent with larger protofibrils (A $\beta-P F)$, a second peak corresponding to intermediate sized oligomers $(\mathrm{A} \beta-\mathrm{iO})$ and a third pool of small oligomers and monomers (Fig. 1Cchromatogram). The effect of each fraction on $20 \mathrm{~S}$ proteasome activity was determined. Only the 
intermediate sized oligomers ( 56kDa) impaired the $20 \mathrm{~S}$ proteasome (Fig. 1C bars) and this impairment correlated with the fractions that were positive for A11 (Fig. 1C dot blot). This inhibitory species also impaired the degradation of fluorogenic substrates specific for each of the 20S's three different proteolytic sites as observed in the mixed oligomer populations (Supplementary Fig. 3A). This suggests that impairment could be due to impairment of substrate entry rather than impairment of a specific catalytic active site. The $A \beta$ protofibril peak $(A \beta-P F)$, lacking the $A 11$ epitope, did not impair degradation of any fluorogenic substrates, even in the presence of ten times more $A \beta$ protofibrils than $A \beta$ intermediate oligomers (Fig. 1D \& Supplementary Fig. 3B).

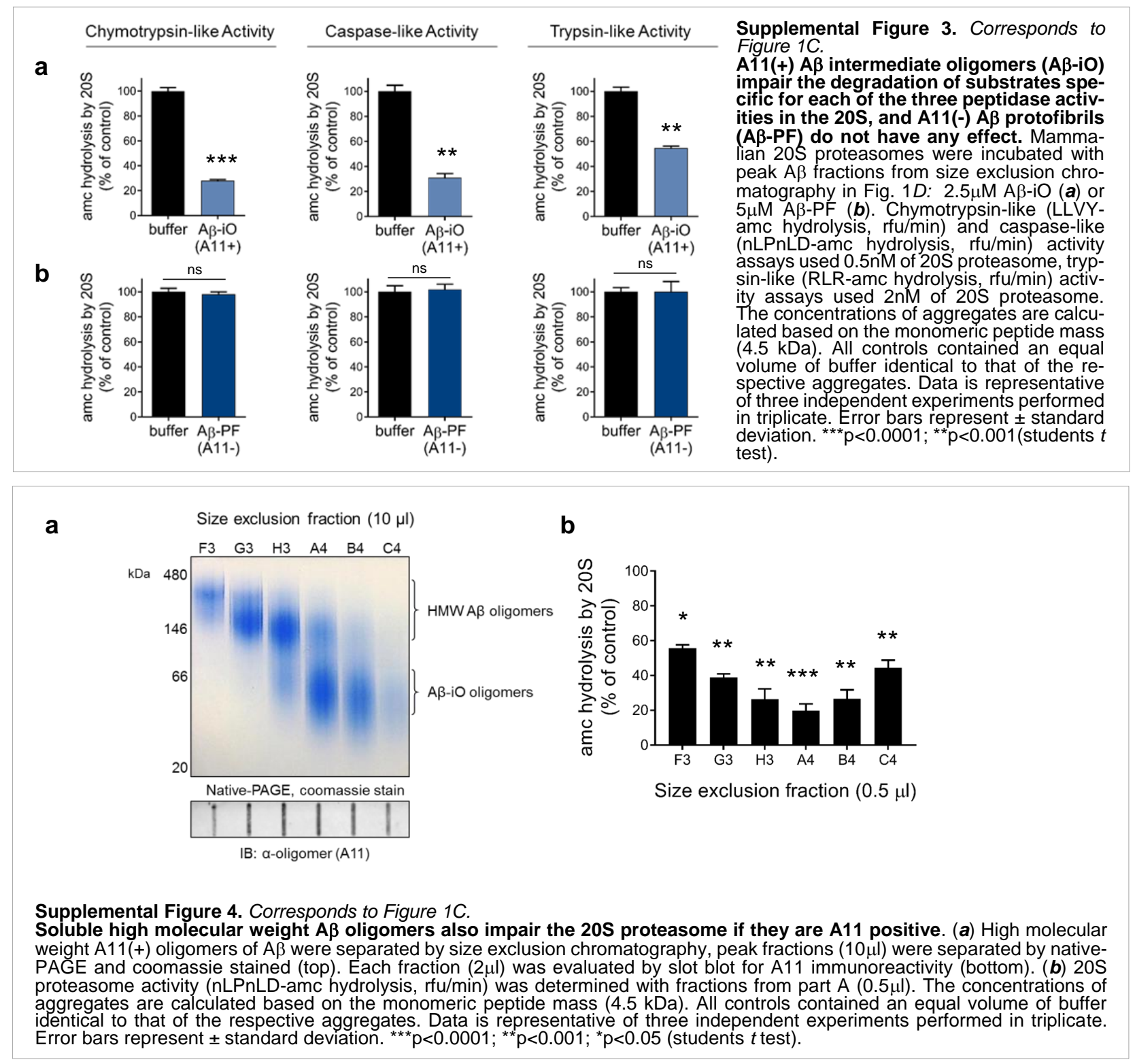


It is plausible that proteasome impairment is due to the oligomer size rather than a specific oligomeric structure. To determine if the impairment is due to the size of the oligomer/protofibrils and whether the shared A11 reactivity is merely a coincidence, we generated high molecular weight (200-400 kDa) A11+A oligomers (Supplementary Fig. 4A) and A11+A $\beta$-protofibrils (>700 kDa) (Supplementary Fig. 5A). The high MW A11+ A $\beta$ oligomers impaired the $20 \mathrm{~S}$ commensurate with the level of $A 11$ reactivity (Supplementary Fig. 4A \& B). The higher molecular weight $A 11+A \beta$ protofibrils also impaired substrate hydrolysis by all three active sites of the proteasome (Supplementary Fig. 5B), although to a considerably lesser extent than the intermediate $A \beta$ oligomers (Fig. 1D). This is expected because protofibrils form when oligomers bind to one another to form a chain of oligomers ${ }^{58}$, which sterically blocks surfaces on the internal oligomers but not the terminal ones, which could still interact with the proteasome. To further determine if the structural epitope of the $A 11$ antibody on the intermediate $A \beta$ oligomers is necessary for proteasome impairment we performed a neutralization assay to block the A11 epitope. A11+ oligomers were incubated with the $A 11$ antibody prior to testing proteasome activity. $A \beta$ oligomers were used at a concentration of $0.2 \mu \mathrm{M}$, the $\mathrm{IC}_{50}$, as determined in Fig. 1D, so an increase or decrease in pro-

a

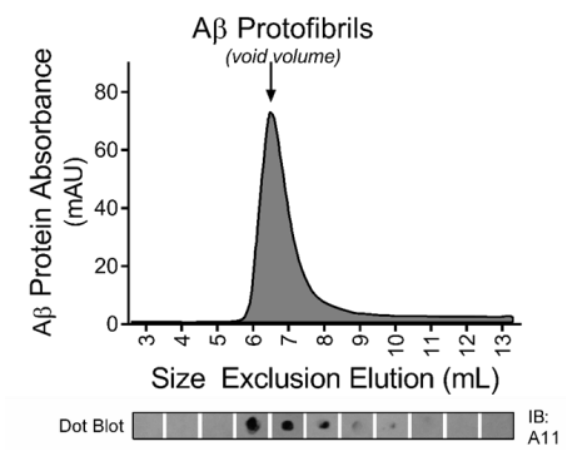

b
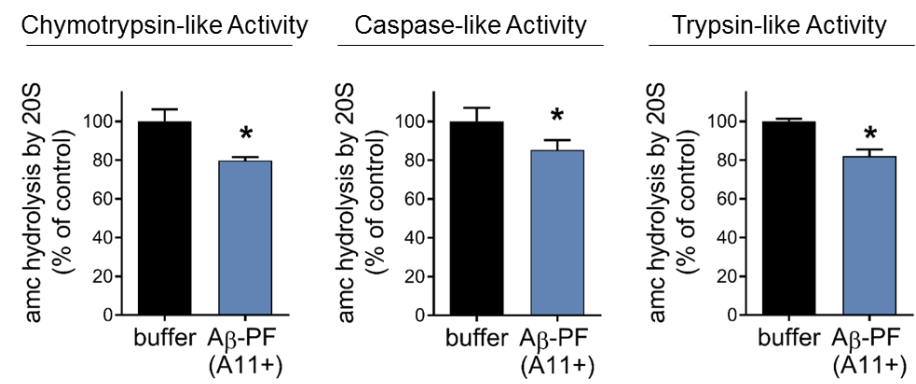

Supplementary Figure 5. Corresponds to Figure $1 \mathrm{C}$.

$A 11(+) A \beta$ protofibrils impair the mammalian $20 S$ proteasome. (a) $A 11(+) A \beta$ protofibril preparation was separated by size exclusion chromatography (Superose $6 \mathrm{GL}$ ). The protofibrils eluted in the column void volume, indicating the soluble aggregates are $>700 \mathrm{kDa}$ (top). Equal volumes of each fraction were probed for A11 reactivity (bottom). (b) Mammalian proteasomes were incubated with $\mathrm{A} 11(+) \mathrm{A} \beta$ protofibrils $(2.5 \mu \mathrm{M})$ and the activity of all three active sites was measured by fluorescent substrate hydrolysis. The concentrations of aggregates are calculated based on the monomeric peptide mass (4.5 kDa). All controls contained an equal volume of buffer identical to that of the respective aggregates. Data is representative of three independent experiments performed in triplicate. Error bars represent \pm standard deviation. ${ }^{\star}=p<0.05$ (student $t$ test). 
teasome activity could be readily observed. Indeed, the A11 antibody when bound to the A $\beta$ oligomers completely rescued proteasome activity (Fig. 1E). As a control, the experiment was repeated with an antibody raised against the $N$-terminal residues of $A \beta$ (clone NAB228), which did not rescue proteasome function (Supplementary Fig. 6). This demonstrates that an available oligomer-specific A11 epitope site is necessary for impairment of the proteasome.

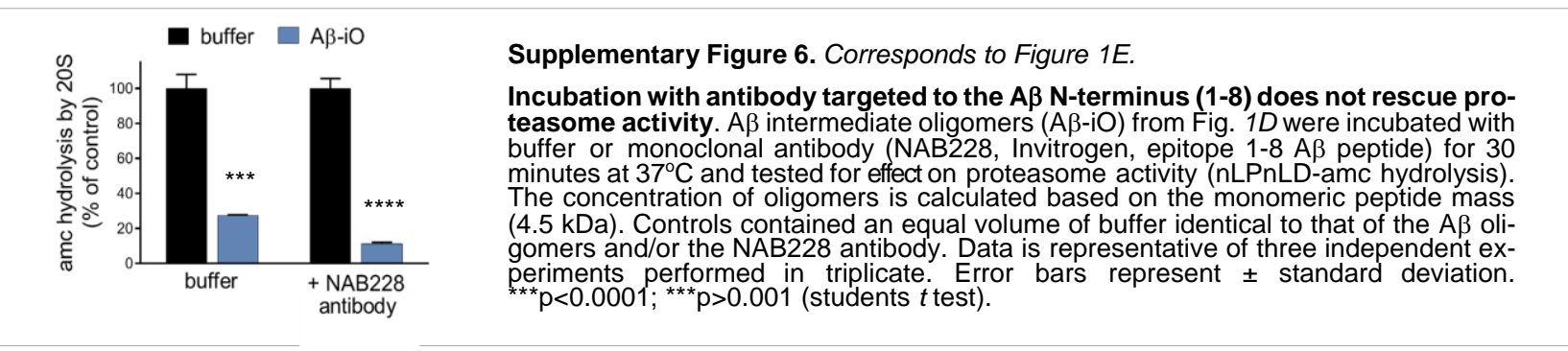

\section{Generation and characterization of homogenous and stable $A 11+A \beta^{\star} 56$ oligomers}

Above we described the isolation of a specific proteasomal inhibitory oligomer from a mixed population of oligomers and aggregates. In order to determine the mechanism of impairment we sought to generate homogenous, stable, and reproducible A11+ oligomers, which could be used for reliable mechanistic analysis. In contrast to $\alpha$-synuclein and huntingtin aggregates, methods to generate physiological relevant oligomers from synthetic $A \beta$ peptides have been extensively developed. Barghorn et al. (2005) ${ }^{59}$ characterized a highly stable A $\beta$ (1-42) oligomer species ( dodecamer) which can be prepared in vitro and can be found in the brains of patients with AD. The relevance of dodecameric $A \beta$ oligomers to disease pathology is established ${ }^{57,60}$. With some modifications to the protocol of Barghorn et al., 2005 we generated A $\beta^{\star} 56$ oligomers, and purified them by nondenaturing size exclusion chromatography (Fig. 2A chromatogram). The major peak corresponds to the intermediate sized $A \beta$ oligomers in Figure 1D. We tested each fraction for proteasome activity and found the major peak impaired the 20S (Fig. 2A bar graph). The single symmetric protein peak demonstrates the homogenous nature of the oligomer preparations. Consistent with $A \beta^{\star} 56$ oligomers isolated from human brain tissue and cerebrospinal fluid, our $A \beta^{\star} 56$ 
oligomers are $\mathrm{A} 11+($ Fig. $2 \mathrm{~A})$, run at $\sim 56 \mathrm{kDa}$ (Fig. 2B) ${ }^{57}$, and significantly impair proteasome activity in a concentration dependent manner (Fig. 2C). Representative real-time fluorogenic substrate hydrolysis data is also shown (Fig 2D).

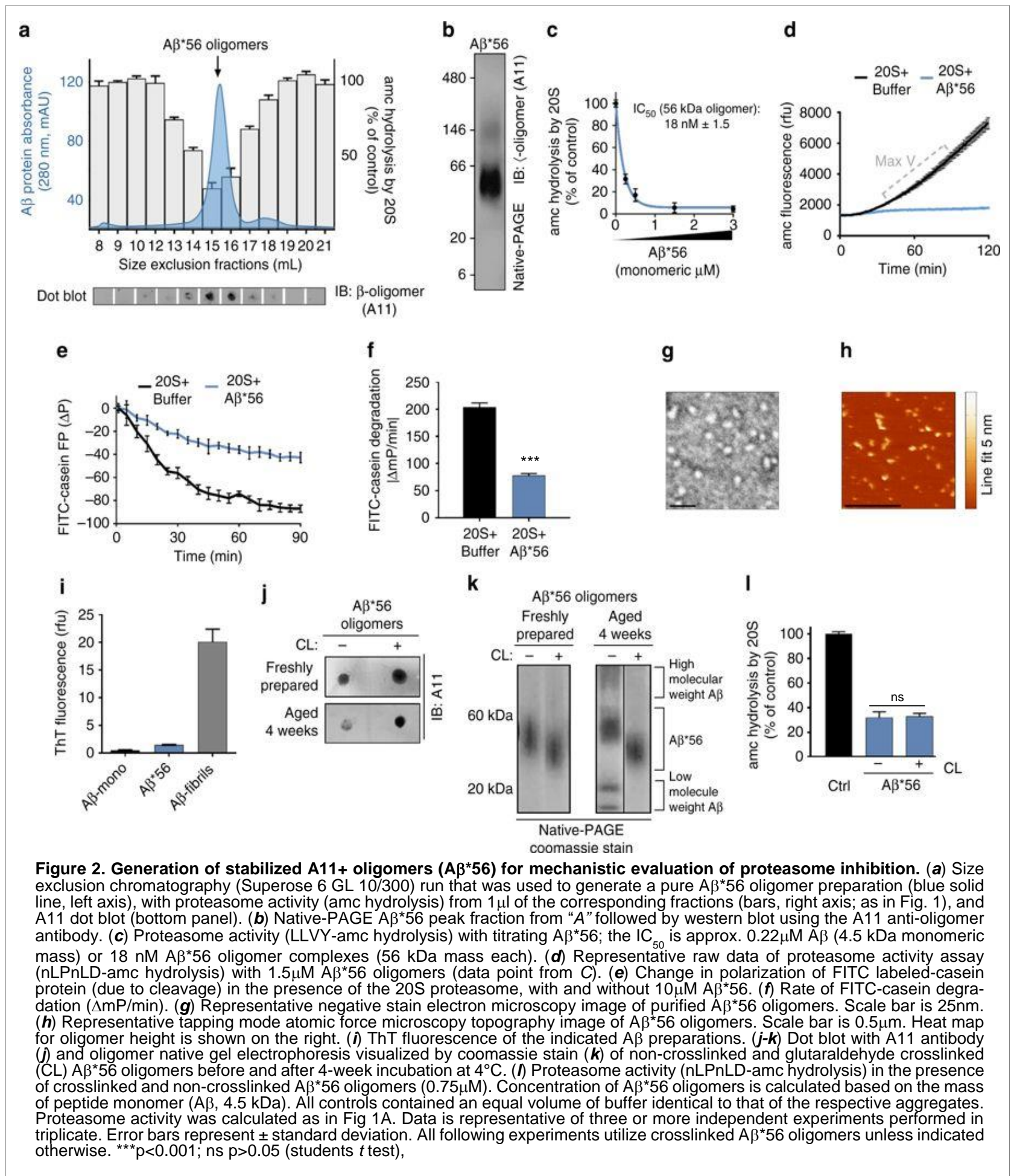


Since $A \beta^{\star} 56$ can impair $20 S$ peptide substrate degradation, we asked if it could impair protein degradation as well. The $20 \mathrm{~S}$ core particle by itself cannot unfold proteins, so we used $\beta$-casein, a classical unfolded protein substrate. We used FITC-labeled casein to follow its degradation in real time using anisotropy, which monitors the tumbling rate of the fluorophore. When FITC-labeled casein is degraded by the proteasome, the tumbling rate of the fluorophore increases, causing a decrease in anisotropy (Fig. 2E 20S+buffer). Similar to peptide substrates, purified $A \beta^{\star} 56$ oligomers also impaired proteasome degradation of the FITC-labeled casein protein (Fig. 2E $\left.20 S+A \beta^{\star} 56\right)$, demonstrating that $A \beta^{\star} 56$ also impairs the degradation of an unfolded protein. We confirmed that the relevant morphology of these oligomers were consistent with those published for synthetic and human brain derived oligomers via: native gel electrophoresis ${ }^{57,61}$ (for MW), TEM $^{62}$ (for spherical shape), AFM ${ }^{63,64}$ (for size), Thioflavin-T staining ${ }^{65}$ (slight but low staining), and anti-oligomer immuno-detection ${ }^{57,65}$ (Fig. 2G-I).

Oligomers are metastable intermediate structures which complicates analysis when consistent homogeneous preparations are needed for in depth biochemical analysis. To circumvent this issue, we stabilized the $A \beta^{*} 56$ oligomers by crosslinking, which maintained the conformation of the A11+ epitope for four weeks when stored at $4^{\circ} \mathrm{C}$ (Fig. 2J) and without crosslinking the A11+ epitope was not as stable over this time period. In addition, the apparent mass of the crosslinked oligomers was also assessed via Native-PAGE and we found that it was unchanged over the fourweek incubation (Fig. 2K). The crosslinked $A \beta^{\star} 56$ oligomers ran slightly faster than the non-crosslinked oligomers as expected ${ }^{59}$, likely due to stabilization of the crosslinked structure. In contrast, the non-crosslinked oligomers partially dissociated into smaller oligomers and formed larger oligomers after four weeks (Fig. 2K). Most importantly, crosslinking of the oligomers does not alter their proteasome impairment activity compared to the non-crosslinked form (Fig. 2L). Together, 


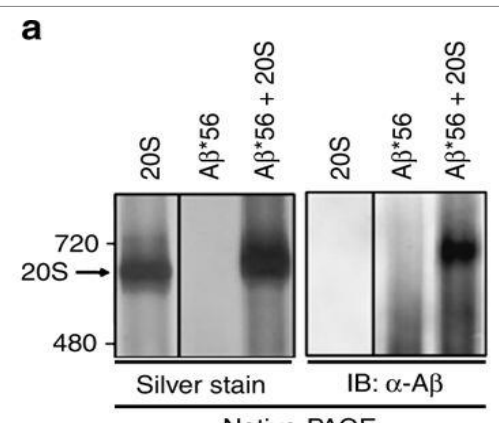

Native-PAGE

b

Chymotrypsin-like activity

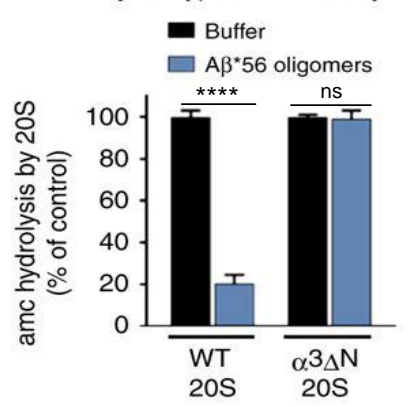

C

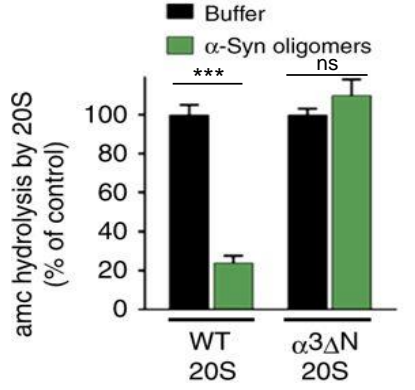

d
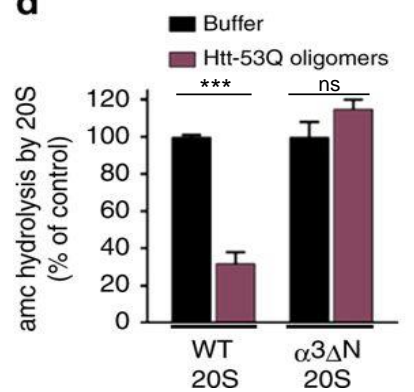

Caspase-like activity

- Buffer

$\square \mathrm{A} \beta^{\star} 56$ oligomers
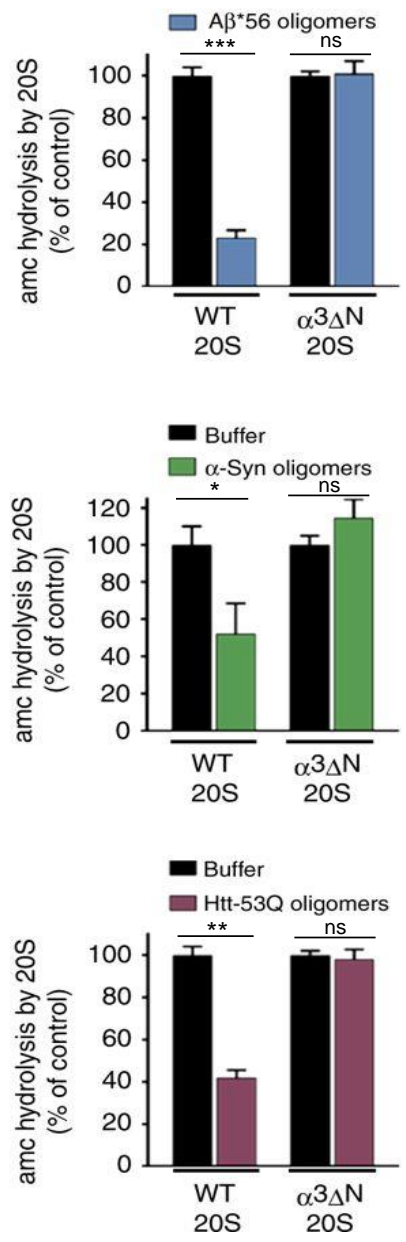
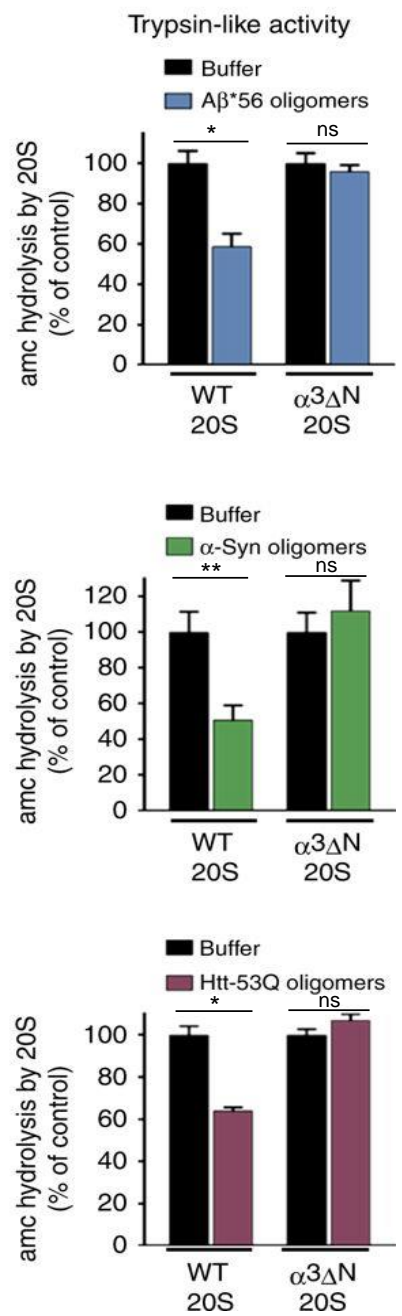

Figure 3. The $A 11(+)$ oligomers bind to the $20 \mathrm{~S}$ proteasome and impair opening of the substrate gate. (a) 20S proteasomes $(0.4 \mu \mathrm{g})$ and pure non-crosslinked $A \beta^{\star} 56$ oligomers $(1.5 \mu \mathrm{g})$ were incubated separately or together for 30 minutes $\left(37^{\circ} \mathrm{C}\right)$, crosslinked with $1 \mathrm{mM}$ glutaraldehyde for 5 minutes, and separated by Native-PAGE (4-8\% tris-acetate gel). Total protein was detected by silver stain (left), and total $A \beta$ was detected by western blot (right). (b-d) The activity of yeast 20S wild-type (WT) and open-gate ( $\alpha 3 \Delta \mathrm{N}$ ) proteasomes was measured for all three proteolytic sites in the presence of $A 11(+)$ oligomers from $A \beta^{\star} 56(B ; 2.5 \mu M), \alpha-S y n(C$; $100 \mathrm{nM})$, and $\mathrm{Htt}-53 \mathrm{Q}(D ; 50 \mathrm{nM})$. Chymotrypsin-like activity was measured by LLVY-amc hydrolysis, trypsin-like activity by RLRamc, and caspase-like by $\mathrm{nLPnLD}$-amc hydrolysis. The concentrations of aggregates are calculated based on the respective monomeric peptide/protein mass. All controls contained an equal volume of buffer identical to that of the respective aggregates. Data is representative of three or more independent experiments performed in triplicate. Error bars represent \pm standard deviation ${ }^{* * * *} \mathrm{p}<0.0001 ;{ }^{* * *} \mathrm{p}<0.001 ;{ }^{* *} \mathrm{p}<0.01 ;{ }^{*} \mathrm{p}<0.05 ;$ ns $\mathrm{p}>0.05$ (students $t$ test).

this demonstrates that the synthetic $A \beta^{\star} 56$ oligomers are homogenous, relevant, stable, reproducible, and represent a single oligomeric species that potently impairs peptide and protein deg- 
radation by the $20 S$ proteasome. These crosslinked $A \beta^{\star} 56$ oligomers are therefore ideally suited for further mechanistic and biochemical analysis to understand how oligomers impair proteasome function and are thus used in all of the following experiments using $A \beta$ oligomers unless stated otherwise.

\section{Direct binding of $A \beta^{\star} 56$ to $20 S$ proteasome}

We next sought to determine if $A \beta^{\star} 56$ and the $20 S$ proteasome could be observed to directly interact. Non-crosslinked $A \beta^{\star} 56$ oligomers were mixed with purified $20 \mathrm{~S}$ proteasomes. To stabilize their interaction, we used a low concentration of glutaraldehyde $(1 \mathrm{mM})$ to induce crosslinking and analyzed migration. $A \beta^{\star} 56$ is clearly seen co-migrating with the $20 S$ proteasome by NativePAGE gel visualized with both silver stain (Fig. 3A left) and by immunoblotting for total A $\beta$ (Fig. 3 A right). Notably, the low concentration of glutaraldehyde treatment did not cause random nonspecific protein crosslinking and did not crosslink the entire multisubunit proteasome into a single $700 \mathrm{kDa}$ complex as determined by the absence of protein aggregates in the SDS-PAGE stacking gel (Supplementary Fig. 7). Minimal crosslinking conditions are further demonstrated by the discrete banding pattern of multiple subunits and the persistence of two single subunit bands (Supplementary Fig. 7).

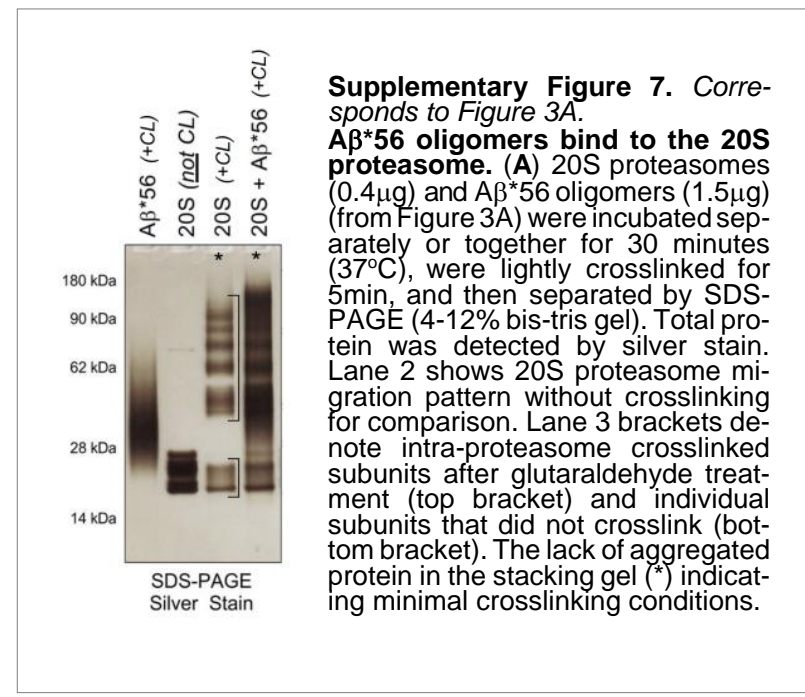

\section{Toxic oligomers impair proteasome gate}

Substrates must pass through the gated translocation channel before gaining access to the proteolytic sites ${ }^{44}$. The $A 11+$ oligomers are too large to enter the $13 \AA$ translocation channel and directly inhibit $\beta$-subunit active sites; however, they could be impairing 20 S proteasome function by impairing substrate entry through 
the gate or by allosterically impairing the active sites. To address this question, we used the $\alpha 3 \Delta \mathrm{N}$ proteasome mutant, which has a constitutively open gate ${ }^{66}$. If the oligomers impair proteasome activity by clogging the catalytic chamber or allosterically impairing the active sites, then they should be able to impair the proteasome regardless if its substrate-gate is in the opened or closed state. Alternatively, if the oligomers require a functioning gate for impairment, then they should not be able to impair a proteasome with a constitutively open gate, i.e. the $\alpha 3 \Delta \mathrm{N} 20 \mathrm{~S}$ proteasome, which lacks only 1 of its $7 \alpha$-subunit $\mathrm{N}$-termini ${ }^{44}$. We added the three different $\mathrm{A} 11+$ oligomers: $\mathrm{A} \beta^{\star} 56, \alpha-S y n$, and $\mathrm{Htt}-53 \mathrm{Q}$, to the WT or the $\alpha 3 \Delta \mathrm{N} 20 \mathrm{~S}$ proteasome and monitored substrate degradation. All three A11 + oligomers significantly impair wild-type proteasomes but do not impair the $\alpha 3 \Delta \mathrm{N}$ proteasomes (Fig. 3B-D). These results demonstrate that the $A 11+$ oligomers require a functioning gate in order to impair the 20 S proteasome. In addition, most active site proteasome inhibitors only inhibit one or two proteolytic sites, but the A11+ oligomers impair the degradation of substrates specific for each of the three different catalytic sites (Fig. 3B-D), further supporting a gating mechanism of impairment, since restricting substrate access would be expected to impair all types of substrates. Moreover, a translocation channel clogging mechanism can also be ruled out since the $\alpha 3 \Delta \mathrm{N} 20$ S proteasome could not be impaired. To further confirm an allosteric mechanism of proteasome impairment, we performed a substrate saturation curve on the WT 20S proteasome with and without $A \beta^{\star} 56$ oligomers. We used non-linear regression and the MichaelisMenten equation to analyze the $K_{D}$ and $V_{\max }$ of the two curves. We found that the $A \beta^{\star} 56$ oligomers caused a decrease in the $V_{\max }\left(49.51\right.$ to $26.48 \mu \mathrm{M}$ substrate) and an increase in the $\mathrm{K}_{\mathrm{m}}(9.816$ to $18.57 \mu \mathrm{M}$ substrate) (Supplementary Fig. 8), which is consistent with allosteric inhibition (i.e. mixed inhibition - a form of noncompetitive inhibition). Taken together, these data clearly demonstrate that all three diseases-related oligomers impair proteasome function by a similar allosteric mechanism, since all three A11+ oligomers require a closable gate on the $20 \mathrm{~S}$ proteasome in order to impair it. 
To validate the preparation of the open-gate $\alpha 3 \Delta \mathrm{N} 20 \mathrm{~S}$ proteasomes, they were incubated with either a known gate-opening peptide (KANLQYYA ${ }^{45}$ from the C-terminus of Rpt5, which includes the HbYX motif) or the $\beta$-subunit active site inhibitor, MG132. Treatment with MG132 completely inhibits WT and open-gate $\alpha 3 \Delta \mathrm{N}$ proteasomes (Supplementary Fig. 9A) as expected for a pure proteasome preparation. The Rpt5 peptide increases WT 20S proteasome substrate degradation but failed to stimulate the open-gate $\alpha 3 \Delta \mathrm{N} 20 \mathrm{~S}$ proteasome (Supplementary Fig. 9B) as expected for proteasomes with constitutively open gates. The preparations of pure $\alpha 3 \Delta \mathrm{N} 20 \mathrm{~S}$ were approximately 10 times more active than the WT 20S and thus ten times more WT 20S was used in these experiments to obtain comparable basal rates (Supplementary Fig. 9).

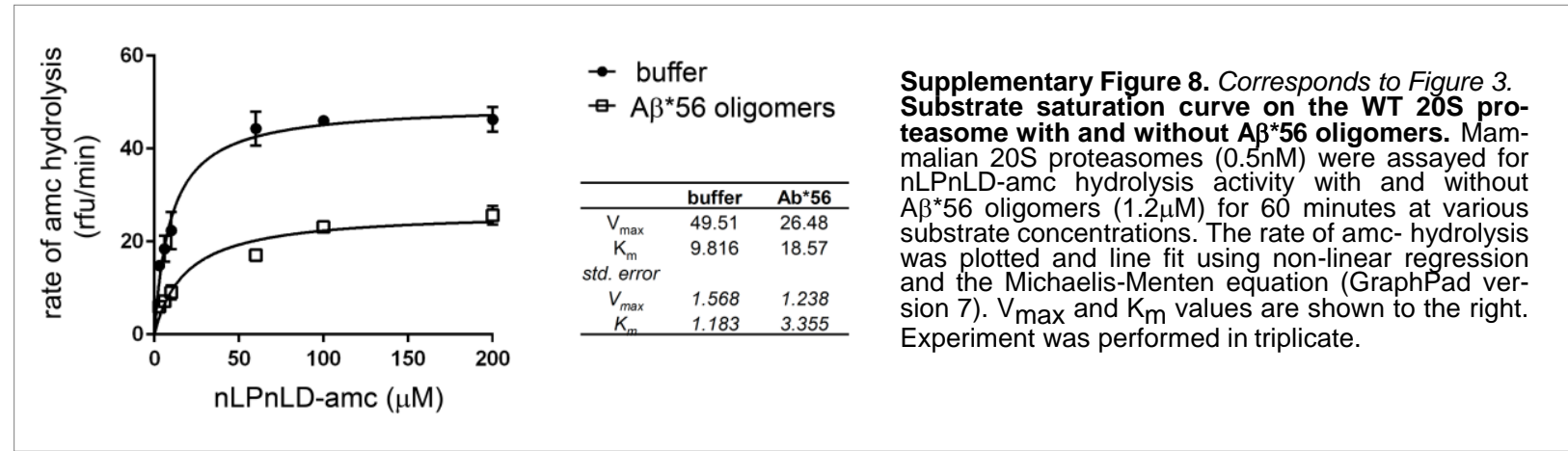

a

WT 20S

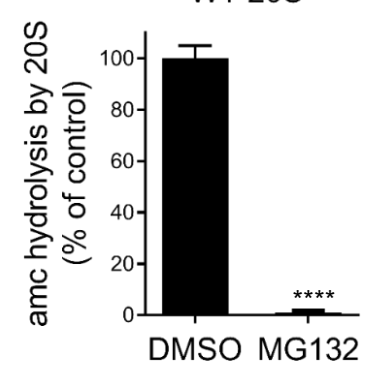

$\alpha 3 \Delta \mathrm{N} 20 \mathrm{~S}$

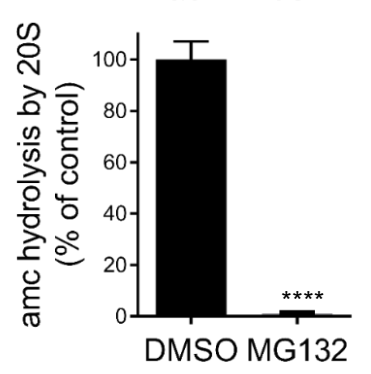

b

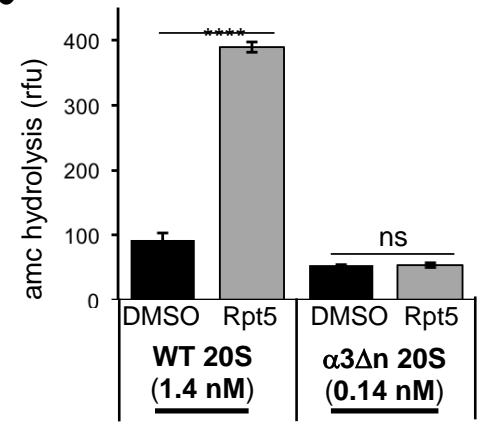

Supplemental Figure 9. Corresponds to Figure $3 B-D$.

WT and a $3 \Delta N$ proteasome preparations have expected activities. (a) Chymotrypsin-like activity (LLVY-amc hydrolysis) is abolished in both wild-type 20S and $\alpha 3 \Delta \mathrm{N} 20 \mathrm{~S}$ mutant proteasomes after pre-treatment with proteasome inhibitor MG132 (50 $\mu M$ ).

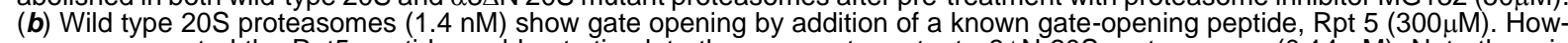
ever, as expected the Rpt5 peptide could not stimulate the open-gate mutant $\alpha 3 \Delta \mathrm{N} 20 \mathrm{~S}$ proteasomes $(0.14 \mathrm{nM})$. Note there is $10 \mathrm{X}$ more WT 20S than $\alpha 3 \Delta \mathrm{N} 20 \mathrm{~S}$. Data is representative of three independent experiments performed in triplicate. Error bars represent \pm standard deviation. ${ }^{\star * * *} p<0.0001 ;$ ns $p>0.05$ (students $t$ test). 


\section{Toxic oligomers stabilize the proteasomes closed gate conformation}

Binding of the 19S ATPases C-termini HbYX motif into the 20S intersubunit pockets induces a conformational change of the $20 \mathrm{~S} \alpha$-subunits, which stabilizes the open state of the $\mathrm{N}$-terminal gating residues ${ }^{9,45}$. However, recent cryo-EM studies have highlighted the complexity of this gateopening mechanism in the $26 \mathrm{~S}$ proteasome when the $19 \mathrm{~S}$ binds to a substrate (or when it is switched from an ATP-bound state to an ATP $\gamma$ S bound state) ${ }^{67,68}$. It is less clear how the dynamics

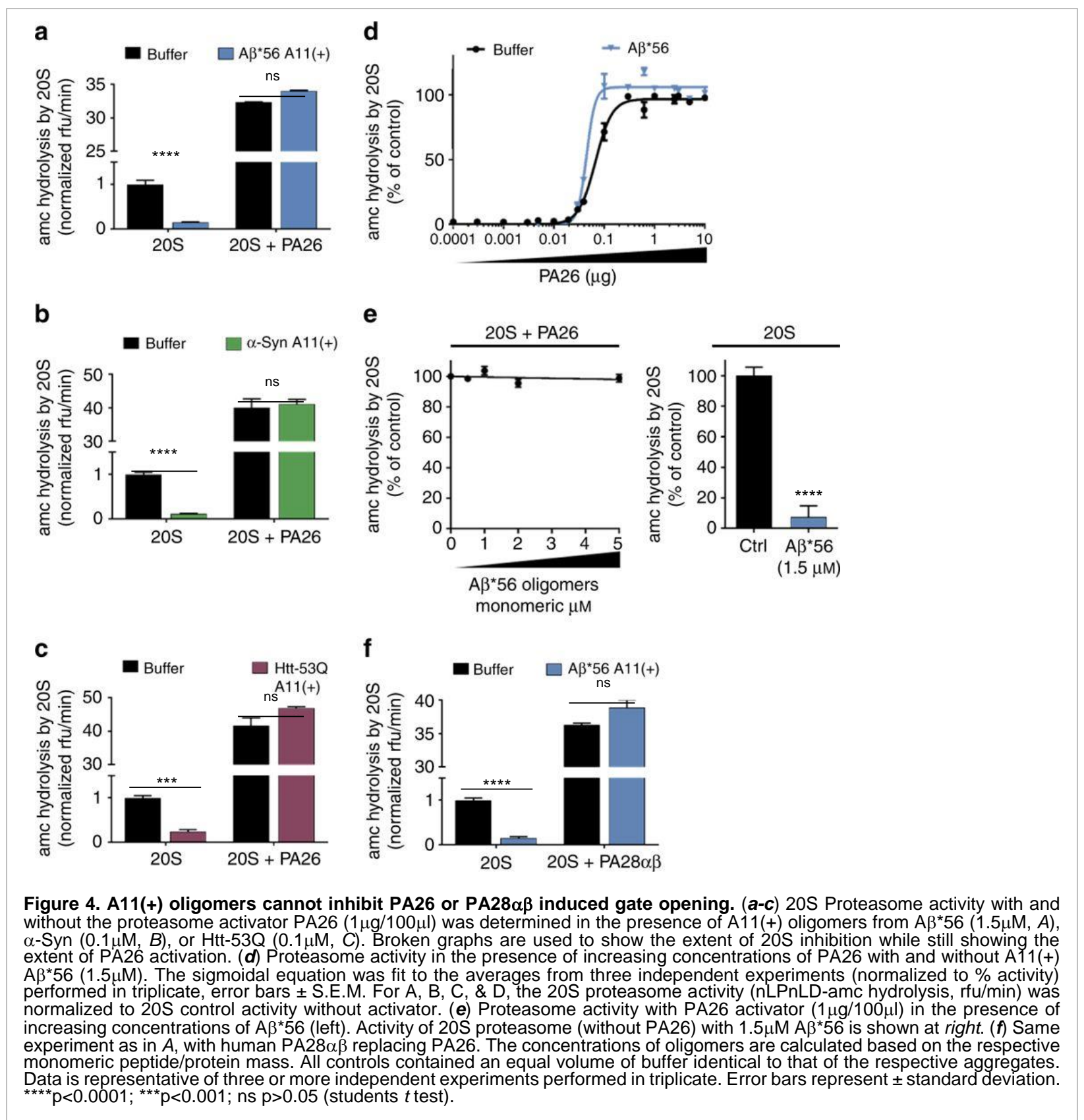


of the gate changes when the 195 associates with the 20S. Nevertheless, functional studies have shown that $19 \mathrm{~S}$ binding to the $20 \mathrm{~S}$ stimulates gate-opening in the $20 \mathrm{~S}$ proteasome ${ }^{39,40}$, in a $\mathrm{HbYX}$ motif dependent manner ${ }^{47}$. In contrast, the 11 S family of proteasome activators (e.g. PA28 $\alpha / \beta$ and PA26) bind to the $20 S \alpha$-subunits and facilitate gate opening by a different mechanism. Although the $11 \mathrm{~S}$ subunits also bind to the $\alpha$-intersubunit pockets, they lack the HbYX motif and thus do not induce $\alpha$-subunit conformational changes like the HbYX motif does. Instead, the 11S internal "activation loop" is required for gate opening. This "activation loop" directly contacts the base of the $\mathrm{N}$-terminal gating residues and locally repositions them into the open conformation ${ }^{69}$. We hypothesized that binding of the A11+ oligomers to the $20 S$ may specifically impair one of these distinct gate-opening mechanisms, which would provide evidence for the mechanism of oligomer-mediated proteasome impairment. If the oligomers could impair the PA26-20S complex, then it is expected that they would bind to the top of the 20S and compete with PA26 for binding to the 20S. Alternatively, if the oligomers do not compete with PA26 for binding to the 20S but they do impair the HbYX dependent gate-opening this indicates that oligomers must allosterically affect conformational changes that are caused upon $\mathrm{HbYX}$ motif binding. Another possibility is that the oligomers affect both or neither mechanisms of gate-opening. To assure that both ends of the 20 S proteasome were bound by PA26 we used saturating amounts to stimulate 20 S peptide degradation. None of the A11+ oligomers from A $\beta, \alpha-S y n$, or Htt-53Q could impair the PA26-20SPA26 complex (Fig. 4A-C). Thus, the PA26-20S-PA26 complex mirrors the results obtained for the $\alpha 3 \Delta \mathrm{N}-20 \mathrm{~S}$. To evaluate this possibility that the oligomers compete with PA26 for binding to the $20 S$ we generated a binding saturation curve for PA26 to $20 S$ by monitoring 20 S proteasome activation in the presence and absence of $A 11+A \beta^{\star} 56$ oligomers. The apparent affinity of PA26 binding for the $20 \mathrm{~S}$ did not decrease in the presence of the A11+ oligomers (Fig. 4D), and the oligomers did not impair PA26 at any concentration that was used, indicating that the oligomers 
do not compete with PA26 for binding to the 20S. Additionally, the A $\beta^{\star} 56$ oligomers could not impair PA26 mediated gate opening even at very high $A \beta^{\star} 56$ concentrations (Fig. 4E).

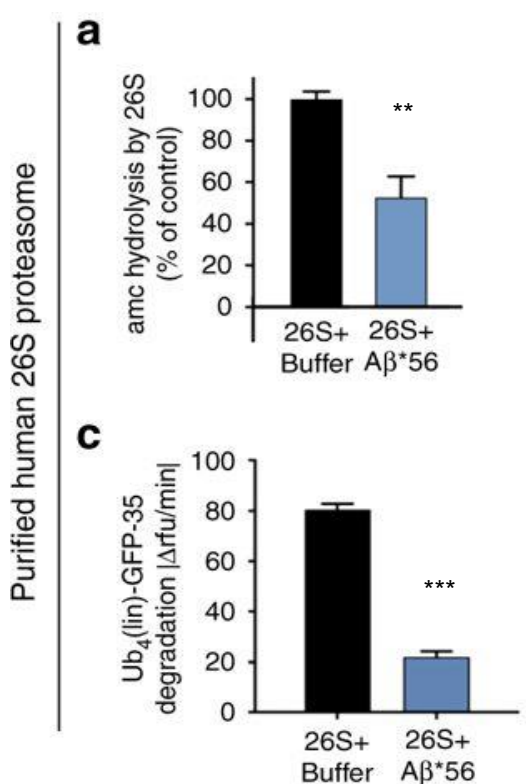

e

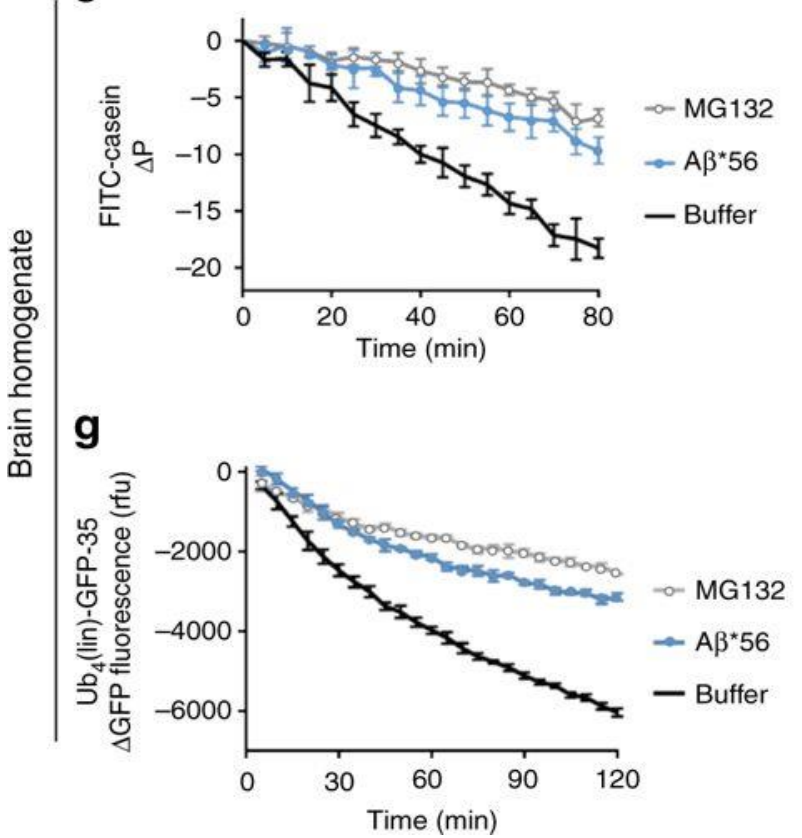

b

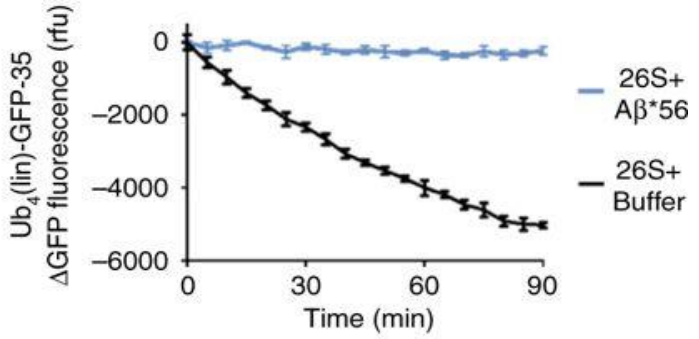

d

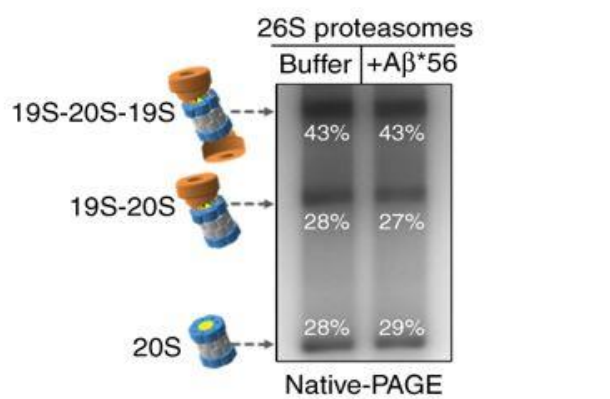

$\mathbf{f}$

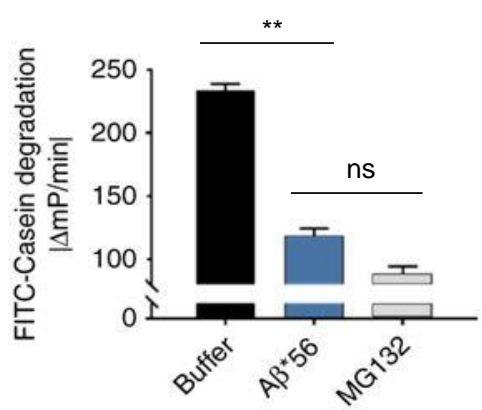

h

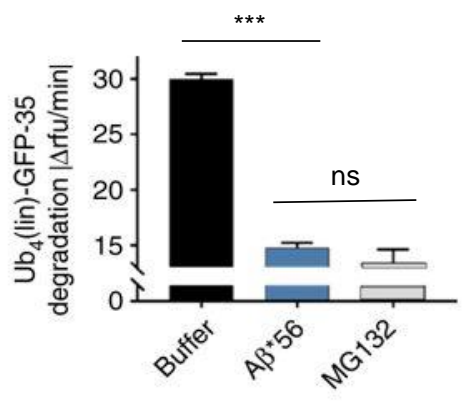

Figure 5. $A \beta^{\star} 56$ oligomers inhibit ubiquitin-dependent and ubiquitin-independent degradation of full-length proteins. (ad) Purified human $26 \mathrm{~S}$ proteasomes. (a) Human $26 \mathrm{~S}$ proteasome activity (LLVY-amc hydrolysis, rfu/min) with $2 \mu \mathrm{M} A \beta^{\star} 56$ compared to buffer control. (b) Change in fluorescence of polyubiquitin-GFP fusion protein (Ub4(lin)-GFP-35) in the presence purified human $26 \mathrm{~S}$ proteasome, with and without $5 \mu \mathrm{M} \mathrm{A} \beta^{*} 56$. (c) Rate of polyubiquitin-GFP fusion protein $\left(\mathrm{Ub}_{4}(\right.$ lin)-GFP-35) degradation in $C(\Delta \mathrm{rfu} / \mathrm{min})$. (d) Purified human $26 \mathrm{~S}$ proteasomes were incubated with or without $A \beta^{\star} 56$ for 90 minutes at $37^{\circ} \mathrm{C}$, and separated by Native-PAGE and silver stained. Band density was quantified with ImageJ. Band density is shown as a percentage of total density of each lane. $(\boldsymbol{e}-\boldsymbol{h})$ Full length protein degradation in mouse brain lysates. (e) Change in polarization of FITC labeledcasein in $2 \mu \mathrm{g}$ mouse brain lysates, with and without $10 \mu \mathrm{M} \mathrm{A} \beta^{*} 56$. ( $\boldsymbol{f}$ Rate of FITC-casein degradation in $E(\Delta \mathrm{mP} / \mathrm{min})$. (g) Change in fluorescence of polyubiquitin-GFP fusion protein ( $\mathrm{Ub}_{4}(\mathrm{lin})$-GFP-35) in $2 \mu \mathrm{g}$ mouse brain lysates, with and without $10 \mu \mathrm{M} A \beta^{\star} 56$. (h) Rate of polyubiquitin-GFP fusion protein ( $\mathrm{Ub}_{4}(\mathrm{lin})$-GFP-35) degradation in $G$ ( $\left.\Delta \mathrm{rfu} / \mathrm{min}\right)$. The concentration of $A \beta^{*} 56$ oligomers is calculated based on $A \beta$ monomeric peptide mass $(4.5 \mathrm{kDa}$ ). All controls contained an equal volume of buffer identical to that of the $A \beta^{\star} 56$ oligomers. Data is representative of three or more independent experiments performed in triplicate. Error bars represent \pm standard deviation. ${ }^{* * *} \mathrm{p}<0.001 ;{ }^{* *} \mathrm{p}<0.01 ; \mathrm{ns} \mathrm{p}>0.05$. (students $t$ test), 
The PA28 $\alpha \beta$ proteasome activator from humans is a homologue of PA26 and thought to open the $20 S$ gate in a similar activation loop-dependent manner ${ }^{69}$. Consistent with PA26 results, the A11+ $A \beta^{\star} 56$ oligomers could not impair the human PA28 $\alpha \beta$ mediated proteasome gate opening (Fig. $4 \mathrm{~F})$. Therefore, the $\mathrm{A} 11+$ oligomers bind to the $20 \mathrm{~S}$ proteasome at a location separate from the 11S proteasome activators, PA26 and PA28 $\alpha \beta$. Based on this we hypothesized that the oligomers stabilize the latent closed conformation of the $\alpha$-subunits which is not affected by the PA26/28 activation loop-dependent gate opening. To test this hypothesis, we asked if the A11+ oligomers could impair peptide and protein degradation by purified human $26 \mathrm{~S}$ proteasomes. The A11+ $A \beta^{\star} 56$ oligomers significantly impaired peptide degradation by the purified $26 S$ proteasome compared to controls (Fig. 5A).

To further test this possibility, we determined if oligomers could impair ubiquitin dependent $\left(\mathrm{Ub}_{4}(\mathrm{lin})\right.$-GFP-35) protein degradation by purified human $26 \mathrm{~S}$ proteasomes. The $\mathrm{Ub}_{4}(\mathrm{lin})$-GFP-35 substrate we used to monitor ubiquitin dependent degradation is a circularly permuted GFP with a linear tetra ubiquitin on $\mathrm{N}$-terminus and a 35-residue unstructured region on the $\mathrm{C}$-terminus that was created in the Matousheck lab. We found that $A \beta^{\star} 56$ also strongly impaired the degradation of this structured protein (Fig. 5B-C) by the human 26S proteasome, which requires ATP-dependent unfolding and injection into the 20S core. These data suggest the oligomers impair the HbYX mechanism of gate opening. However, it is possible that the oligomer binding to the $20 \mathrm{~S}$ could cause the 26S to disassemble into its $20 S$ and $19 S$ subcomplexes, which could also have the effect of impairing the ubiquitin-dependent protein degradation that we observed. To test this possibility, we incubated $A \beta^{\star} 56$ oligomers and purified human $26 S$ proteasome preparations together for 90 minutes at $37^{\circ} \mathrm{C}$ before running the samples on native-PAGE (Fig. 5D). We quantified the silver stain band densities for isolated 20S, singly capped 26S, and doubly capped $26 \mathrm{~S}$. The relative ratio of these three populations of proteasomes did not change with the incubation with A $\beta$ compared to control. 
The prior experiments where done with highly purified components thus providing good cause and effect confidence for mechanistic analysis; however, the purified system cannot assess if the oligomers are able to bind to and impair the proteasome in an environment that more closely mimics a complex cellular environment. To address this, we prepared mouse brain lysates to determine if the toxic oligomers could still impair protein degradation by the proteasome in such a heterogeneous environment. We found that the brain lysates were highly competent to degrade the protein substrates FITC-casein (Fig. 5E-F) and Ub4 (lin)-GFP-35 proteins (Fig. 5G-H) similar to the purified $26 \mathrm{~S}$ proteasome (Fig. 5B-C). We also assessed the proteasome activity component of this lysate by adding the proteasome inhibitor MG132, and found that the majority of the degradation activity we observed was due to proteasome activity (Fig. 5E-H).

When we assessed the degradation of these two specific proteins in brain lysates, in the presence of the crosslink-stabilized $A \beta^{\star} 56$ we observed extensive proteasome impairment—nearly as much as when MG132 was used (Fig. 5E-H). Therefore, the $A 11+A \beta^{\star} 56$ oligomers retain enough specificity to bind to and nearly completely impair proteasome function even in a complex brain lysate. These data demonstrate that the oligomers do not disrupt the $26 \mathrm{~S}$ complexes, and thus do not impair it by this mechanism, but must instead act, as we expected, on the gate. Impairment of the $26 \mathrm{~S}$ proteasome also demonstrates that the oligomers must bind to the $20 \mathrm{~S}$ even in the presence of the 19S, as was also observed for the PA26-20S complexes, demonstrating that the oligomers likely bind to the outer surface of the $20 \mathrm{~S}$ proteasome (i.e. not on the gating surface) thus supporting the hypothesis that these toxic oligomers act allosterically preventing the $20 \mathrm{~S}$ gate from opening properly. Since these results clearly demonstrate that A11+ oligomers are able to impair the $20 \mathrm{~S}$ core particle by itself, then it's most likely that their impairment of the $26 \mathrm{~S}$ proteasome is via the same mechanism, acting on the core particle. Importantly, this result demonstrates that A11+ oligomers can also impair proteasome function in a complex protein environment. 
The 195 requires binding of ATP for it to bind to and induce gate opening in the $20 S^{73}$. The $26 S$ proteasome adopts multiple conformations during the ATP hydrolysis cycle and substrate degradation ${ }^{67,74-76}$. In the presence of hydrolysable ATP, 26 S proteasomes seems to alternate between active (open gate) and inactive (closed gate) states, with the inactive state predominating, and in contrast, non-hydrolyzing ATP analogues better stabilize the active (open gate) form of the proteasome ${ }^{67,77}$. Interestingly, while the $A \beta^{\star} 56$ oligomers impaired the $26 S$ in the presence of ATP, they could not impair the $26 \mathrm{~S}$ in the presence of the analog ATP $\gamma \mathrm{S}$ (Fig. 6A). This shows that the oligomers are able to impair the normal physiological (with ATP) state of the $26 \mathrm{~S}$ but not the
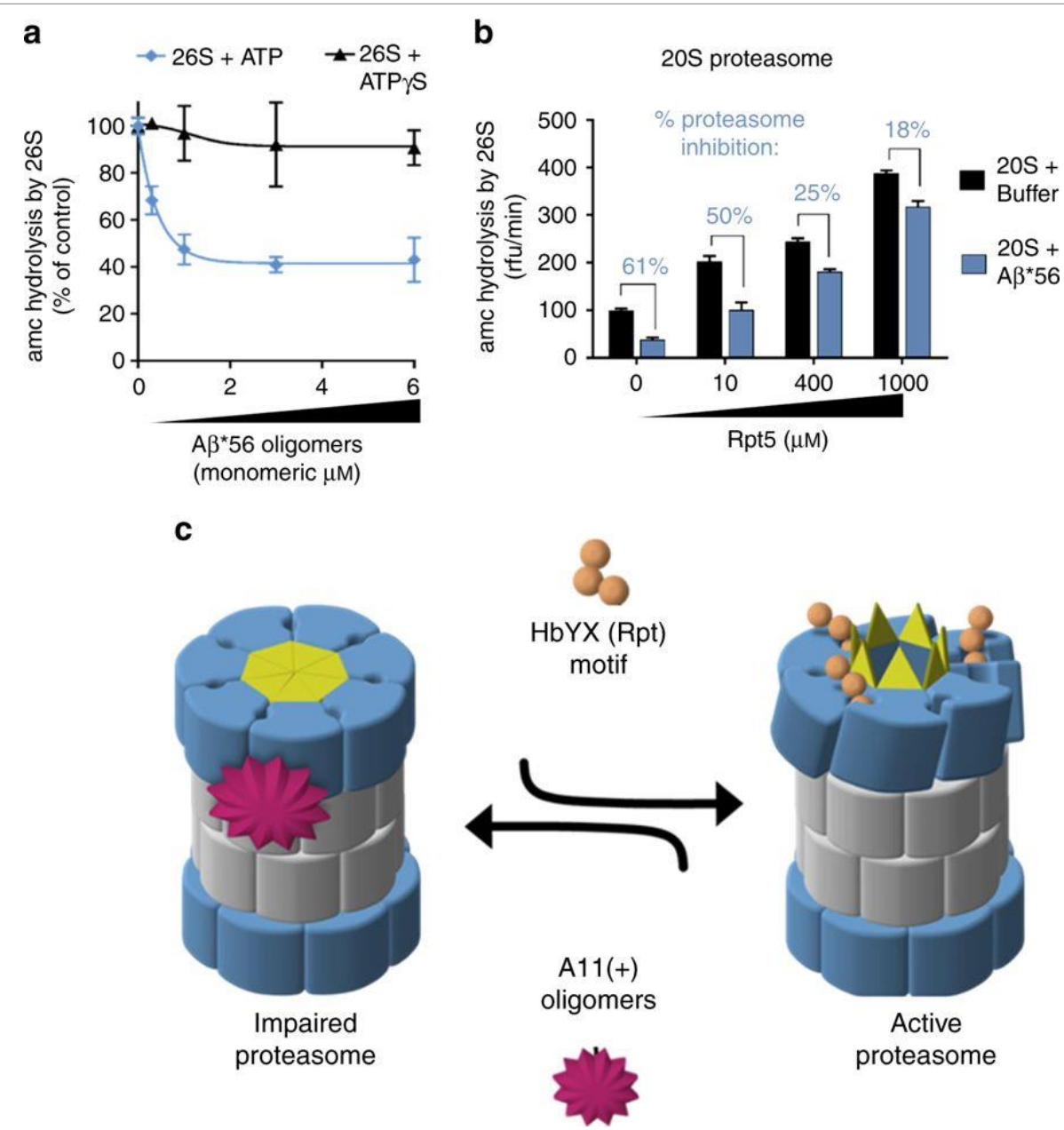

Figure 6. HbYX-dependent $20 \mathrm{~S}$ gate opening counteracts inhibition by $A \beta^{\star} 56$ oligomers.

(a) Mammalian $26 \mathrm{~S}$ proteasome activity (LLVY-amc hydrolysis, rfu/min) with $2 \mathrm{mM}$ ATP or $10 \mu \mathrm{M}$ ATP $\gamma$ S. A $\beta^{\star} 56$ titration up to $6 \mu \mathrm{M}$. (b) $20 \mathrm{~S}$ proteasome activity (nLPnLD-amc hydrolysis, rfu/min) with RPT5 peptide titration, with and without $\mathrm{A} \beta^{\star} 56(0.5 \mu \mathrm{M})$. (c) Schematic depicting our working model for proteasome inhibition by $A 11$ (+) oligomers (see text for details). The concentration of $A \beta^{\star} 56$ oligomers is calculated based on $A \beta$ monomeric peptide mass ( $\left.4.5 \mathrm{kDa}\right)$. All controls contained an equal volume of buffer identical to that of the $A \beta^{\star} 56$ oligomers. Data is representative of three or more independent experiments performed in triplicate. Error bars represent \pm standard deviation. 


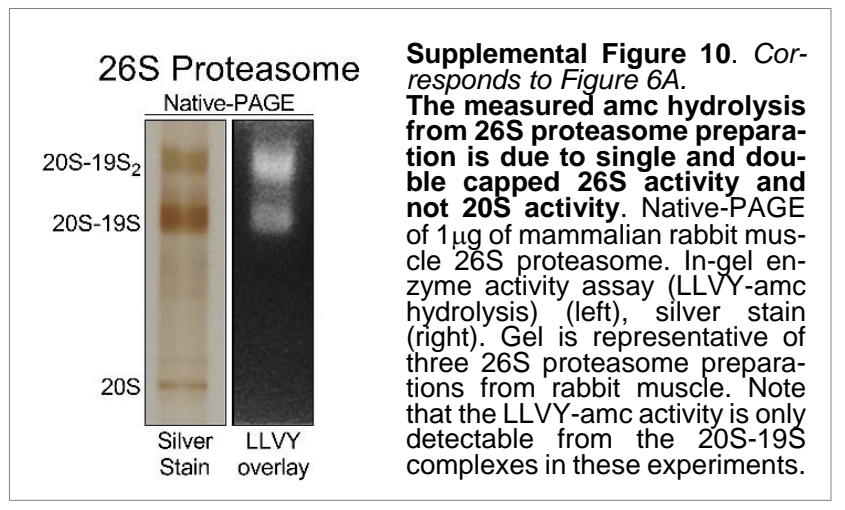

synthetically opened state (using ATP $\gamma \mathrm{S}$ ), in which the open state is more "strongly" stabilized. We verified the integrity of the purified 265 proteasomes preparation via NativePAGE to confirm that the observed activity came only from the $26 \mathrm{~S}$ complexes (Supple-

mentary Fig. 10) and not from any free $20 \mathrm{~S}$ proteasome in the preparation. These results thus further support the hypothesis that these oligomers oppose the HbYX-dependent conformational changes that lead to gate-opening.

To further test this hypothesis, we asked if the A11+ oligomers could block HbYX-dependent gate opening directly by the Rpt5 peptide (KANLQYYA), an established gate opening peptide ${ }^{45}$, derived from the C-terminus of Rpt5. We added increasing concentrations of the Rpt5 peptide to the $20 S$ proteasome with and without the $A 11+A \beta^{\star} 56$ oligomers. In the absence of oligomers, the Rpt5 peptide significantly stimulated proteasome activity as expected. However, the oligomers impaired Rpt5 activation at all concentrations. Interestingly, the more Rpt5 was added the less effective the oligomers were to impair the proteasome (Fig. 6B). These results indicate that the oligomers impair HbYX dependent gate opening, but also that HbYX peptide could overcome impairment by the oligomers at the higher concentrations (1mM Rpt5 peptide was the highest concentration that could be tested due to its solubility). In contrast, the oligomers could not impair PA26 activation at any concentration of PA26. We interpret these results to mimic the ATP/ATY $\gamma$ S experiment (Fig. 6A) whereby the ATP state is a low HbYX occupancy state and the ATP $\gamma \mathrm{S}$ state is a higher occupancy HbYX state. The rational is that ATP is rapidly hydrolyzed to ADP, and ADP cannot support HbYX-dependent gate-opening. On the other hand, ATP $\gamma \mathrm{S}$ is not hydrolyzed to ADP and thus it sustains the HbYX bound open-gate state or it could also enhance gate-opening by other mechanisms ${ }^{77}$. These combined results fit well with a model whereby the $A 11+$ oligomers 
impair proteasome function by binding to the outer surface of the $20 \mathrm{~S}$ barrel, and impair substrate entry by allosterically stabilizing the closed conformational state of the $20 S \alpha$-subunits, in a way that directly counteracts the conformational changes that are required for $\mathrm{HbYX}$-dependent gateopening.

\section{Discussion}

The structural evolution of compartmentalized proteases was driven by the need to protect proteolytic activity from the cellular milieu, but still have the capacity to degrade select proteins in a regulated manner. The substrate-entry gate in the $20 \mathrm{~S}$ proteasome thus plays a critical role in proteasome function and in cellular proteostasis. Here, we elucidate a common mechanism whereby soluble oligomers possessing a common three-dimensional structure found in many neurodegenerative diseases potently inhibit $20 S$ and 265 proteasome gate-opening thus drastically impairing its function. While certain studies show some forms of aggregates do not impair the proteasome (which we also find Fig. 1 B \& D), the aggregates from these studies were not assayed for the presence of A11+ oligomers. Based on our results we proposed the following mechanistic model (Fig. 6C) of how A11+ oligomers impair proteasome function: 1) A11+ oligomers bind with low nM affinity (Fig. $1 \& 2$ ) to the outer surface of the $\alpha$-subunits along the C2 axis (the presumed binding site); 2) by binding to this site the oligomers stabilize the closed conformation of the $\alpha$-subunits and prevent spontaneous gate-opening (Fig. 3); 3) activation loop-dependent gate opening (e.g. PA26) occurs normally in the presence of oligomers, since its mechanism only requires contact between the activation loops and the base of the gating residues (Fig. 4); 4) however, HbYX dependent gate-opening (e.g. the 19S regulatory particle or HbYX peptide) is inhibited as oligomer-bound $\alpha$-subunits are unable to undergo the conformational changes required to open the gate (Fig. $5 \& 6$ ), which are stabilized by the bound oligomer. From a general mechanistic perspective, in this model one expects to observe opposing allosteric controls fought between two allosteric modulators that bind to distinct sites on the $20 \mathrm{~S}$ proteasome. From this 
model, one expects to observe competition between two allosteric modulators (the HbYX motif and the oligomers) that bind to distinct sites on the 20S proteasome. In this sense, the HbYX motif is a positive allosteric modulator that induces gate opening, whereas the A11+ oligomers are negative allosteric modulators that induce gate closing. These diametrically opposed regulators thus fight to control the proteasome gate. Moreover, it appears that the HbYX mechanism is dominant since binding of the non-hydrolysable ATP analog, ATP $\gamma \mathrm{S}$, prevents inhibition by A11+ oligomers (Fig. 6A), though further confirmation is warranted.

These results demonstrate that oligomer-mediated impairment of proteasome function is not dependent on the sequence of the misfolded protein but rather the oligomer's three-dimensional shape. Specifically, we found a consistent correlation between an oligomer's ability to impair the proteasome and recognition by the $\mathrm{A} 11$ antibody. While the physiological concentration of $\mathrm{A} 11+$ oligomers in neurons is unknown, if we consider that the affinity constant for the oligomers is low $\mathrm{nM}$, and that the cellular concentration of the $20 \mathrm{~S}$ is estimated to be low micromolar ${ }^{70}$ then, with respect to this binding reaction, the $20 \mathrm{~S}$ is saturating in the cell. This implies toxic oligomers will bind to the $20 \mathrm{~S}$ irrespective of their cellular concentration. Which begs the question: are physiological levels of A11+ oligomers sufficient to impact protein degradation? Using laser capture microdissection and isolation of hippocampal pyramidal neurons from sporadic Alzheimer's Disease cases, Hashimoto et al. (2010) determined the intraneuronal concentration of $A \beta 42$ to be $3 \mu \mathrm{M}^{71}$, but what proportion of the intracellular $A \beta 42$ is in oligomeric form is not known. Furthermore, Kisselev et. al. (2006) showed that the amount of proteasome inhibitor, Velcade ${ }^{\mathrm{TM}}$ that is used to treat multiply myeloma only inhibits protein degradation by about $10-25 \%{ }^{72}$. This result demonstrates that a relatively small alteration of protein breakdown can have a substantial impact on cell death. Consistent with this reasoning, stereotaxic unilateral infusion of lactacystin (a selective proteasome inhibitor) into the substantia nigra pars compacta of rats caused neurodegenerative disease like symptoms ${ }^{22}$. However, the percentage of proteasomes that must be active in 
neurons to maintain normal proteostasis is not known and thus we could only speculate about what level of intracellular A11 oligomers would be required to impact neuronal function. Nevertheless, as protein degradation begins to suffer as oligomers accumulate, the level of proteasome impairment is expected to increase exponentially as more proteins accumulate and oligomerize. Such a model would be expected to exhibit exponential progression kinetics, which coincides with the exponential deterioration that is observed over decades in most neurodegenerative diseases. These results build confidence that such oligomers in neurons could impair proteasome function enough to contribute to the progression of these neurodegenerative diseases.

Future efforts are required to understand which structures within the A11 epitope facilitate $20 S$ proteasome binding and impairment and if this phenomenon occurs in human disease conditions. Elucidation of this mechanism provides a compelling model to explain why proteasome function has been found to be impaired in virtually all neurodegenerative diseases. Interestingly, Choi et. al. $(2016)^{78}$ showed that opening of the 20 S proteasome gate in cells leads to enhanced cellular proteasome function, including ubiquitin-dependent protein degradation, decreased protein aggregates, and protection from oxidative stress. Our model provides a mechanistic framework to develop small molecules to counteract proteasome impairment via A11+ oligomers. Illustrating this potential mechanism of proteasome impairment identifies novel drug targets for developing small molecule activators of the proteasome gate. Such therapeutic interventions have the potential to restore proteostasis in patients suffering from neurodegenerative diseases. 


\section{Acknowledgments}

We thank the members of the Smith lab for helpful and valuable discussions, and the protein core at WVU for their services. We thank Dr. Andreas Matouschek and his lab for generously providing us with the $\mathrm{Ub}^{4}$ (lin)-GFP ${ }^{35}-\mathrm{His}^{6}$ plasmid, Dr. Olivier Coax for kindly providing the PA28 $\alpha \beta$ plasmid, and Dr. William Wonderlin for kindly providing the $\alpha$-Synuclein plasmid. We thank Dr. Justin Legleiter for use of his AFM equipment and kindly providing the Htt-53Q and Htt-20Q constructs.

\section{Author Contributions}

T.A.T. designed, performed, and analyzed the various experiments in this manuscript (with input from D.M.S.). T.A.T. purified most proteins used in the manuscript, R.T.A. purified the Ub ${ }^{4}($ lin)GFP substrate. T.A.T and R.T.A. performed the $\alpha$-Synuclein experiments. Results were analyzed and interpreted by T.A.T., R.T.A., and D.M.S. Manuscript preparation was done by T.A.T. and D.M.S. All authors reviewed the results and approved the final version of this manuscript.

\section{Competing financial interests}

The authors declare no competing financial interests. 


\section{Materials and Methods}

\section{Proteasome purifications}

Mammalian 20S proteasomes were isolated from bovine liver as described ${ }^{79}$. Briefly, cleared liver homogenate was passed over DE53 column. Protein was eluted with a stepwise $\mathrm{NaCl}$ gradient. Fractions with significant proteasome activity were pooled and further separated by a strong anion exchange column (ResourceQ, GE Healthcare) eluting with $\mathrm{NaCl}$ gradient. Fractions with high suc-LLVY-amc hydrolysis were pooled for further purification using a hydroxyapatite column (CHT-I, Bio-Rad) and eluted by $\mathrm{KPO}_{4}$ gradient. Fractions with high proteasome activity were pooled and further purified by SEC (S-400, GE Healthcare). Eluted fractions were pooled and purity of 20 S proteasomes (>98\%) was determined by SDS-PAGE and quantified by densitometry (ImageJ, NIH). Mammalian 26S proteasomes were isolated from rabbit muscle using the Ubl affinity purification as described ${ }^{80}$. Human $26 \mathrm{~S}$ proteasomes were affinity purified on a streptavidin column from the HEK293- $\beta 4$-biotin cell line as described ${ }^{78}$. Recombinant PA26 was expressed in BL21-STAR E. coli and purified by affinity with a Ni-NTA column (Qiagen), as described ${ }^{81}$. Recombinant human PA28 $\alpha \beta$ was expressed in BL21-STAR E. coli and purified by affinity with a NiNTA column (Qiagen), as described ${ }^{82}$. Wild-type and mutant $\alpha 3 \Delta \mathrm{N}$ yeast $20 \mathrm{~S}$ proteasomes were expressed and purified by anion-exchange chromatography as described ${ }^{83}$. Fluorogenic substrate peptides were purchased from BostonBiochem (suc-LLVY-amc) and EZBiolabs (acnLPnLD-amc and ac-RLR-amc). Rpt5 peptides were synthesized by EZBiolabs. Protein concentrations were determined by Bradford assay (BSA standard) (Thermo Scientific).

\section{Proteasome activity assays - peptide substrates}

Unless otherwise specified, bovine $20 \mathrm{~S}(0.5 \mathrm{nM})$, rabbit muscle $26 \mathrm{~S}(0.4 \mathrm{nM})$, yeast WT $20 \mathrm{~S}(1.4$ $\mathrm{nM})$, or yeast $\alpha 3 \Delta \mathrm{N} 20 \mathrm{~S}(0.14 \mathrm{nM})$ proteasomes were assayed using fluorogenic peptides, as 
described ${ }^{45}$ in 96 -well black flat bottom untreated plates (Costar). Briefly, proteasomes were incubated in a reaction buffer containing $50 \mathrm{mM}$ Tris $-\mathrm{HCl}(\mathrm{pH} 7.4)$, and $100 \mu \mathrm{M}$ fluorogenic substrate (suc-LLVY-amc, ac-nLPnLD-amc) or $10 \mu \mathrm{M}$ fluorogenic substrate (boc-LRR-amc). 20S proteasomes were treated with Rpt5, or with PA28 $\alpha \beta$ or PA26 to induced gate opening as indicated. Rabbit muscle $26 \mathrm{~S}$ proteasomes were used in the presence of $1 \mathrm{mM} \mathrm{DTT,} 10 \mathrm{mM} \mathrm{MgCl}$, and $100 \mu \mathrm{M}$ of fluorogenic substrate (ac-nLPnLD-amc) with either $2 \mathrm{mM}$ ATP $(99 \%$, Sigma) or $10 \mu \mathrm{M}$ ATP $\gamma$ S (95\%, Sigma). Fluorescence was measured every 55 s for 120 min (ex/em:380nm/460nm). The rate of increase in fluorescence intensity is directly proportional to proteasome activity. For all experiments, an equal volume of the appropriate control buffer (identical to the aggregate/oligomer buffer that is described below) was used for controls. All molar concentrations of $\mathrm{A} \beta, \alpha-\mathrm{Syn}$, and $\mathrm{Htt}-53 \mathrm{Q}$ are calculated based upon the monomeric protein concentration.

\section{Proteasome activity assays - protein substrates}

FITC-casein $\left(0.08 \mu \mathrm{g}\right.$, Sigma) and $\mathrm{Ub}_{4}(\mathrm{lin})$-GFP-35 $(0.08 \mu \mathrm{g}$, a kind gift from Dr. Andreas Matousheck) degradation assays were carried out in $50 \mu$ l reactions using 96-half-well non-binding surface treated black plates (Corning) at $37^{\circ} \mathrm{C}$. The GFP substrate was generated as described ${ }^{84}$. Proteasomes were added to the reactions $(1 \mu \mathrm{g} 20 \mathrm{~S}$, or $0.9 \mu \mathrm{g}$ human $26 \mathrm{~S})$ in the presence or absence of $A \beta^{\star} 56$ oligomers $(10 \mu \mathrm{M})$ and fluorescence was measured at every 60 seconds for 90 minutes. Data shown is the mean of three reactions, with a 5-point moving average, and error bars represent +/- standard deviation. Degradation rates were determined by calculating the slope of a line fit to the first 30 minutes of activity.

\section{A 1 1-42 peptide}

Synthetic $A \beta(1-42)$ was purchased from Selleckchem, Anaspec, and EZBiolabs. To remove preexisting aggregates, synthetic A $\beta$ peptide was dissolved in 100\% Hexafluoroisopropanol 
(HFIP), and incubated at $37^{\circ} \mathrm{C}$ for 2 hours with shaking (500 RPM). The HFIP was removed and the remaining peptide films were stored at $-80^{\circ} \mathrm{C}$ until use. Monomeric $A \beta$ was obtained by dissolving synthetic peptide in $100 \%$ anhydrous DMSO (Thermo Scientific) at $5 \mathrm{mM}$ and diluted with PBS to a final concentration of $50 \mu \mathrm{M}$ immediately prior to use. Crude $\mathrm{A} \beta$ aggregates were prepared as described ${ }^{85}$. A $\beta^{\star} 56$ oligomers were generated similar to Barghorn et al. $2005^{59}$. Briefly, HFIP treated peptide films were resuspended in 100\% anhydrous DMSO $(5 \mathrm{mM})$ and bath sonicated for twenty minutes before further dilution $(400 \mu \mathrm{M})$ with $20 \mathrm{mM} \mathrm{NaPO}_{4} \mathrm{pH} 7.4,140 \mathrm{mM} \mathrm{NaCl}$, $0.2 \%$ SDS. The $100 \mu \mathrm{M} \mathrm{A} \beta$ was incubated at $37^{\circ} \mathrm{C}$ for six hours, diluted to $100 \mu \mathrm{M}$ with nanopore $\mathrm{H}_{2} \mathrm{O}$, incubated at $37^{\circ} \mathrm{C}$ for 18 hours, centrifuged for 10 minutes at $3,000 \mathrm{~g}$, and the supernatant containing $A \beta^{\star} 56$ oligomers was removed and dialyzed against $5 \mathrm{mM} \mathrm{NaPO}_{4} \mathrm{pH} 7.4,35 \mathrm{mM} \mathrm{NaCl}$. Where indicated, $A \beta^{*} 56$ oligomers were crosslinked (before dialysis) with $1 \mathrm{mM}$ glutaraldehyde (EM grade, Thermo Scientific) for 2 hours at room temperature. The reaction was quenched by the addition of $1 \mathrm{M}$ Tris- $\mathrm{HCl} \mathrm{pH} 8$ (to a final concentration of $10 \mathrm{mM}$ ) and incubated for an additional 30 minutes. $A \beta^{\star} 56$ oligomers were purified by SEC (Superose 12 10/30, GE Healthcare) and eluted as a single major peak. Each preparation of $A \beta^{\star} 56$ was confirmed to be $A 11+$ by dot blot analysis as described below. To generate $A \beta-H M W A 11+$ oligomers, the second $A \beta$ incubation at $100 \mu \mathrm{M}$ was extended to 26 hours. To generate $A \beta A 11$ + protofibrils, the second $A \beta$ incubation at $100 \mu \mathrm{M}$ was extended to 50 hours. The $\mathrm{A} \beta \mathrm{A} 11$ + protofibrils eluted from the Superose 6 column in a single peak at the void volume and were confirmed to be $>700 \mathrm{kDa}$ by Native-PAGE. All buffers were filtered with $0.2 \mu \mathrm{m}$ membranes immediately prior to use. All SEC experiments were performed on an ÄKTApurifier (GE Healthcare) at $4^{\circ} \mathrm{C}$ with $5 \mathrm{mM} \mathrm{NaPO}_{4} \mathrm{pH} 7.4,35 \mathrm{mM} \mathrm{NaCl}$ at a flow rate of $0.5 \mathrm{~mL} / \mathrm{min}$. $A \beta$ concentration was calculated by UV absorption at $280 \mathrm{~nm}$ (molar extinction coefficient $1940 \mathrm{M}^{-1} \mathrm{~cm}^{-1}$ ) and confirmed with Bradford protein concentration assay (Thermo Scientific). 


\section{$\alpha-S y n u c l e i n$ protein}

Human wild-type $\alpha$-Synuclein ( $\alpha$-Syn) with $\mathrm{N}$-terminal his-tag in pET28a vector was expressed and purified from BL21-STAR E. coli using a Ni-NTA column (Qiagen) followed by anion exchange chromatography (HiTrapQ, GE Healthcare). Pure $\alpha$-Syn monomers were obtained by SEC (Superose 12 10/30, GE Healthcare) immediately prior to use. The purity of $\alpha$-Syn monomers (>98\%) was determined by SDS-PAGE and quantified by densitometry (ImageJ, NIH). Crude $\alpha$-Syn aggregates/oligomers were generated by incubating monomeric $\alpha$-Syn $(3 \mathrm{mg} / \mathrm{mL})$ in PBS $(20 \mathrm{mM}$ $\left.\mathrm{NaPO}_{4} \mathrm{pH} 7.4,140 \mathrm{mM} \mathrm{NaCl}\right)$ at $37^{\circ} \mathrm{C}$ for 7 hours. After oligomerization, the oligomers were separated from the remaining monomers by SEC (Superose 12, GE Healthcare) and verified A11+ by dot blot.

\section{Huntingtin protein}

GST-tagged huntingtin exon1 constructs with a 53 polyglutamine repeat (GST-Htt-53Q) and a 20 polyglutamine repeat (GST-Htt-20Q). Protein was expressed and purified from BL21-STAR E. coli as described ${ }^{86}$. Briefly, the GST-fusion protein was cleaved with PreScission Protease (GE Healthcare) at $4^{\circ} \mathrm{C}$ according to manufacturer protocol. The free $\mathrm{Htt}-53 \mathrm{Q}$ proteins were further purified by SEC (Superose 12 10/30, GE Healthcare) to obtain a monomeric population immediately prior to oligomerization. The purity of $\mathrm{Htt}-53 \mathrm{Q}$ monomers (>95\%) was analyzed with SDSPAGE quantified by densitometry (ImageJ, NIH). Oligomers were generated by incubating monomeric $\mathrm{Htt}-53 \mathrm{Q}(1 \mathrm{mg} / \mathrm{mL})$ at $37^{\circ} \mathrm{C}$ for $1 \mathrm{hr}$. Due to the rapid formation of Htt-53Q oligomers, monomeric Htt-20Q (which oligomerized at a much slower rate) was used for the monomer assay in Figure 1B.

\section{Crude aggregate fractionation}

Insoluble aggregates were removed from crude aggregate preparations by centrifugation at $10,000 \times g$ for 10 minutes. The supernatant containing soluble oligomers was transferred to a 
fresh Eppendorf tube and the remaining pellet was gently resuspended in PBS. The pellet fraction was centrifuged twice more before final resuspension at $1 \mathrm{mg} / \mathrm{mL}$ in PBS. The fibrillar nature of the insoluble fraction was confirmed by Thioflavin-T (Sigma) fluorescence in comparison to monomer preparation controls as described below.

\section{SDS-PAGE and Native-PAGE}

Proteins were separated by SDS-PAGE using NuPAGE ${ }^{\mathrm{TM}}$ 4-12\% Bis-Tris Protein Gels (Invitrogen), or separated by Native-PAGE using Novex ${ }^{\text {TM }}$ 10-20\% Tris-Glycine or NuPAGE ${ }^{\text {TM }} 3-8 \%$ TrisAcetate Protein Gels (Invitrogen), as indicated. Total protein was visualized with coomassie stain (Simply Blue Safe Stain, Novex) or silver stain (Pierce Silver Stain kit, Thermo Scientific) as indicated according to manufacturer instructions. Immunoblots were performed as described below. Native-PAGE in-gel 26 s proteasome activity assay was performed using NuPAGE ${ }^{\text {TM }} 3-8 \%$ TrisAcetate gels (Invitrogen). Samples were mixed with Novex ${ }^{\mathrm{TM}}$ Tris-Glycine Native Sample Buffer (2X) (Invitrogen) just before loading. Electrophoresis was carried out in Novex ${ }^{\mathrm{TM}}$ Tris-Glycine Native Running Buffer (Invitrogen) (with $0.5 \mathrm{mM}$ DTT, $1 \mathrm{mM} \mathrm{ATP}$, and $5 \mathrm{mM} \mathrm{MgCl}_{2}$ ) at $4^{\circ} \mathrm{C}$ and $150 \mathrm{~V}$ for 4 hours. Native gels containing $26 \mathrm{~S}$ proteasomes were incubated with reaction buffer ( $50 \mathrm{mM}$ Tris $\mathrm{pH} 7.5,10 \mathrm{mM} \mathrm{MgCl} 2,2 \mathrm{mM}$ ATP, $1 \mathrm{mM}$ DTT, $50 \mu \mathrm{M}$ suc-LLVY-AMC) for $30 \mathrm{~min}$ at $37^{\circ} \mathrm{C}$. Fluorescent bands around proteasomes were visualized by standard gel-imaging systems for DNA staining by ethidium bromide.

\section{Immunoblotting}

For Native-PAGE Western blots, proteins were transferred to nitrocellulose membrane (GE) using tris-glycine transfer buffer (Novex). Primary antibodies were purchased from Invitrogen (anti-oligomer A11, and anti-A $\beta$ N-terminus clone NAB228) and diluted 1:1000 in TBST (50mM tris, 150 $\mathrm{mM} \mathrm{NaCl}, 0.01 \%$ Tween-20) $+5 \%$ nonfat milk prior to use. AlexaFluor-647 conjugated secondary antibodies (Invitrogen) were diluted 1:3500 in TBST prior to use. Membranes were blocked for 1 hour at room temperature in TBST $+10 \%$ nonfat milk, briefly washed with TBST, incubated with 
primary antibody for 1 hour at room temperature, washed with TBST ( $3 \times 5$ minutes), incubated with secondary antibody for 1 hour at room temperature, washed ( $3 \times 5$ minutes), and imaged on a Molecular Dynamics Typhoon 9410 Variable Mode Imager. Dot blots were performed by spotting protein on $0.1 \mu \mathrm{M}$ nitrocellulose membranes and processed the same as Western blots.

\section{Antibody neutralization assays}

Anti-oligomer A11 (Invitrogen) and A $\beta$ N-terminal antibody (clone NAB228, Invitrogen) were buffer exchanged to $50 \mathrm{mM}$ Tris ( $\mathrm{pH} 7.4$ ) with Zeba spin desalting columns (Thermo Scientific). The antibodies $(0.5 \mu \mathrm{g})$ were incubated with $\mathrm{A} \beta^{\star} 56(50 \mu \mathrm{M})$ or control buffer for 25 minutes at $37^{\circ} \mathrm{C}$ before adding to proteasome activity assays.

\section{Crosslinking $A \beta^{\star} 56$ and 20 S proteasomes}

Mammalian $20 \mathrm{~S}$ proteasomes were buffer exchanged to $10 \mathrm{mM} \mathrm{NaPO}_{4}(\mathrm{pH} 7$ with Zeba spin desalting columns (Thermo Scientific) and incubated with $A \beta^{\star} 56$ oligomers (or an equal volume of control buffer) for 45 minutes at $37^{\circ} \mathrm{C} .1 \mathrm{mM}$ Glutaraldehyde was added to the mixture of $20 \mathrm{~S}$ proteasomes and $A \beta^{\star} 56$ oligomers and incubated for 5 minutes at $37^{\circ} \mathrm{C}$. Crosslinking reactions were quenched by the addition of $1 \mathrm{M}$ Tris- $\mathrm{HCl} \mathrm{pH} 8(1 \mathrm{mM})$. Proteins were separated by SDSPAGE and Native-PAGE and visualized with silver stain or immunoblotting as described above.

\section{Oligomer characterization}

For atomic force microcopy imaging, preformed $A \beta$ oligomers were deposited on freshly cleaved mica (Ted Pella Inc., Redding, CA) and allowed to sit for $30 \mathrm{~s}$. The mica substrate was then washed with $200 \mu \mathrm{L}$ of ultrapure water and dried with a gentle stream of nitrogen. Samples were imaged in tapping mode via ex situ AFM using a Nanoscope V MultiMode scanning probe microscope (Veeco, Santa Barbara, CA). AFM images were analyzed with Matlab equipped with the image processing toolbox (Mathworks, Natick, MA). For negative stain electron microscopy, $6 \mu l$ 
of preformed $A \beta^{\star} 56$ oligomers were applied to ultra-thin copper 400 mesh carbon grids (Electron Microscopy Sciences) and imaged on a JEOL JEM-2100 Transmission Electron Microscope.

\section{Thioflavin-T florescence measurement}

Thioflavin-T (ThT) (Sigma) was dissolved (1mM) in PBS, filtered through a $0.2 \mu \mathrm{M}$ syringe filter, and stored at $-20^{\circ} \mathrm{C}$ until use. For the assays, $3 \mu \mathrm{g}$ of $A \beta$ was incubated at room temperature for 10 minutes in $100 \mu \mathrm{M}$ of PBS with $20 \mu \mathrm{M}$ ThT and fluorescence was measured (ex/em: 450nm/490nm) in a Synergy2 plate reader (GenTek).

\section{Statistical analysis}

Data were analyzed using an unpaired Student's t-test (Prism). For all statistical analyses, a value of $p<0.05$ was considered significant. 


\section{References}

1. Brettschneider, J., Tredici, K. Del, Lee, V. M.-Y. Y. \& Trojanowski, J. Q. Spreading of pathology in neurodegenerative diseases: a focus on human studies. Nat. Rev. Neurosci. 16, 109-20 (2015).

2. Rubinsztein, D. C. The roles of intracellular protein-degradation pathways in neurodegeneration. Nature 443, 780-786 (2006).

3. Selkoe, D. J. Folding proteins in fatal ways. Nature 426, 900-904 (2003).

4. Haass, C. \& Selkoe, D. J. Soluble protein oligomers in neurodegeneration: Lessons from the Alzheimer's amyloid beta-peptide. Nat. Rev. Mol. Cell Biol. 8, 101-112 (2007).

5. Kayed, R. \& Lasagna-Reeves, C. A. Molecular mechanisms of amyloid oligomers toxicity. Adv. Alzheimer's Dis. 3, 67-78 (2012).

6. Calamini, B. \& Morimoto, R. I. Protein homeostasis as a therapeutic target for diseases of protein conformation. Curr. Top. Med. Chem. 12, 2623-40 (2012).

7. Labbadia, J. \& Morimoto, R. I. The biology of proteostasis in aging and disease. Annu. Rev. Biochem. 84, 435-464 (2015).

8. Ciechanover, A. Intracellular protein degradation: From a vague idea through the lysosome and the ubiquitin-proteasome system and onto human diseases and drug targeting. Bioorganic Med. Chem. 21, 3400-3410 (2013).

9. Glickman, M. H. \& Ciechanover, A. The ubiquitin-proteasome proteolytic pathway: Destruction for the sake of construction. Physiol. Rev. 82, 373-428 (2002).

10. Ciechanover, A. \& Kwon, Y. T. Degradation of misfolded proteins in neurodegenerative diseases: therapeutic targets and strategies. Exp. Mol. Med. 47, e147 (2015).

11. Tai, H.-C. \& Schuman, E. M. Ubiquitin, the proteasome and protein degradation in neuronal function and dysfunction. Nat. Rev. Neurosci. 9, 826-38 (2008).

12. Fonseca, R. et al. A balance of protein synthesis and proteasome-dependent degradation determines the maintenance of LTP. Neuron 52, 239-245 (2006).

13. Djakovic, S. N., Schwarz, L. A., Barylko, B., DeMartino, G. N. \& Patrick, G. N. Regulation of the proteasome by neuronal activity and calcium/calmodulin-dependent protein kinase II. J. Biol. Chem. 284, 26655-26665 (2009).

14. Djakovic, S. N. et al. Phosphorylation of Rpt6 regulates synaptic strength in hippocampal neurons. J. Neurosci. 32, 5126-5131 (2012).

15. Lopez-Salon, M. et al. The ubiquitin-proteasome cascade is required for mammalian longterm memory formation. Eur. J. Neurosci. 14, 1820-1826 (2001).

16. Ramachandran, K. V \& Margolis, S. S. A mammalian nervous-system-specific plasma membrane proteasome complex that modulates neuronal function. Nat. Struct. Mol. Biol. 24, 419-430 (2017).

17. Ciechanover, A. \& Brundin, P. The ubiquitin proteasome system in neurodegenerative diseases: Sometimes the chicken, sometimes the egg. Neuron 40, 427-446 (2003).

18. Keller, J. N., Hanni, K. B. \& Markesbery, W. R. Impaired proteasome function in Alzheimer's disease. J. Neurochem. 75, 436-439 (2000).

19. McNaught, K. S., Olanow, C. W., Halliwell, B., Isacson, O. \& Jenner, P. Failure of the ubiquitin-proteasome system in Parkinson's disease. Nat. Rev. Neurosci. 2, 589-594 (2001).

20. McKinnon, C. \& Tabrizi, S. J. The ubiquitin-proteasome system in neurodegeneration. Antioxid. Redox Signal. 5, 2302-2321 (2014).

21. Ortega, Z., Díaz-Hernández, M. \& Lucas, J. J. Is the ubiquitin-proteasome system impaired in Huntington's disease? Cellular and Molecular Life Sciences 64, 2245-2257 (2007).

22. McNaught, K. S. P. et al. Proteasome inhibition causes nigral degeneration with inclusion bodies in rats. Neuroreport 13, 1437-1441 (2002). 
23. McNaught, K. S. P., Belizaire, R., Jenner, P., Olanow, C. W. \& Isacson, O. Selective loss of $20 \mathrm{~S}$ proteasome alpha-subunits in the substantia nigra pars compacta in Parkinson's disease. Neurosci Lett 326, 155-158 (2002).

24. Xie, W. et al. Proteasome inhibition modeling nigral neuron degeneration in Parkinson's disease. J. Neurochem. 115, 188-199 (2010).

25. Bedford, L. et al. Depletion of $26 \mathrm{~S}$ proteasomes neurodegeneration and lewy-like inclusions resembling human pale bodies. J. Neurosci. 28, 8189-8198 (2008).

26. Li, Z., Arnaud, L., Rockwell, P. \& Figueiredo-Pereira, M. E. A single amino acid substitution in a proteasome subunit triggers aggregation of ubiquitinated proteins in stressed neuronal cells. J. Neurochem. 90, 19-28 (2004).

27. Cecarini, V. et al. Amyloid peptides in different assembly states and related effects on isolated and cellular proteasomes. Brain Res. 1209, 8-18 (2008).

28. Díaz-Hernández, M. et al. Inhibition of $26 \mathrm{~S}$ proteasome activity by huntingtin filaments but not inclusion bodies isolated from mouse and human brain. J. Neurochem. 98, 1585-1596 (2006).

29. Gregori, L., Fuchs, C., Figueiredo-Pereira, M. E., Van Nostrand, W. E. \& Goldgaber, D. Amyloid beta-protein inhibits ubiquitin-dependent protein degradation in vitro. J. Biol. Chem. 270, 19702-19708 (1995).

30. Lindersson, E. et al. Proteasomal inhibition by alpha-synuclein filaments and oligomers. J. Biol. Chem. 279, 12924-12934 (2004).

31. Bence, N. F., Sampat, R. M. \& Kopito, R. R. Impairment of the ubiquitin-proteasome system by protein aggregation. Science. 292, 1552-1555 (2001).

32. Oh, S. et al. Amyloid peptide attenuates the proteasome activity in neuronal cells. Mech. Ageing Dev. 126, 1292-1299 (2005).

33. Tanaka, Y. et al. Inducible expression of mutant alpha-synuclein decreases proteasome activity and increases sensitivity to mitochondria-dependent apoptosis. Hum. Mol. Genet. 10, 919-926 (2001).

34. Tseng, B. P., Green, K. N., Chan, J. L., Blurton-jones, M. \& Laferla, F. M. A $\beta$ inhibits the proteasome and enhances amyloid and tau accumulation. Neurobiol. Aging 29, 1607-1618 (2008).

35. Emmanouilidou, E., Stefanis, L. \& Vekrellis, K. Cell-produced $\alpha$-synuclein oligomers are targeted to, and impair, the $26 \mathrm{~S}$ proteasome. Neurobiol. Aging 31, 953-968 (2010).

36. Deriziotis, P. et al. Misfolded PrP impairs the UPS by interaction with the $20 \mathrm{~S}$ proteasome and inhibition of substrate entry. EMBO J. 30, 3065-3077 (2011).

37. Kristiansen, M. et al. Disease-associated prion protein oligomers inhibit the $26 \mathrm{~S}$ proteasome. Mol. Cell 26, 175-188 (2007).

38. DeMartino, G. N. et al. Identification, purification, and characterization of a PA700dependent activator of the proteasome. J. Biol. Chem. 271, 3112-3118 (1996).

39. Adams, G. M., Crotchett, B., Slaughter, C. A., DeMartino, G. N. \& Gogol, E. P. Formation of proteasome-PA700 complexes directly correlates with activation of peptidase activity. Biochemistry 37, 12927-12932 (1998).

40. Chu, P. M. et al. Identification, purification, and characterization of a high molecular weight, ATP-dependent activator (PA700) of the $20 \mathrm{~S}$ proteasome. J Biol Chem 269, 3539-3547 (1994).

41. DeMartino, G. N. \& Slaughter, C. A. The proteasome, a novel protease regulated by multiple mechanisms. J Biol Chem 274, 22123-22126 (1999).

42. Voges, D., Zwickl, P. \& Baumeister, W. The 26S proteasome: a molecular machine designed for controlled proteolysis. Annu. Rev. Biochem. 68, 1015-1068 (1999).

43. Groll, M. et al. Structure of $20 \mathrm{~S}$ proteasome from yeast at 2.4 A resolution. Nature 386, 463-471 (1997).

44. Groll, M. et al. A gated channel into the proteasome core particle. Nat. Struct. Biol. 7, 1062- 
1067 (2000).

45. Smith, D. M. et al. Docking of the proteasomal ATPases' carboxyl termini in the $20 \mathrm{~S}$ proteasome's $\alpha$ ring opens the gate for substrate entry. Mol. Cell 27, 731-744 (2007).

46. Rabl, J. et al. Mechanism of gate opening in the $20 \mathrm{~S}$ proteasome by the proteasomal ATPases. Mol. Cell 30, 360-368 (2008).

47. Gillette, T. G., Kumar, B., Thompson, D., Slaughter, C. A. \& DeMartino, G. N. Differential roles of the $\mathrm{COOH}$ termini of AAA subunits of PA700 (19S regulator) in asymmetric assembly and activation of the $26 \mathrm{~S}$ proteasome. J. Biol. Chem. 283, 31813-31822 (2008).

48. Finley, D., Chen, X. \& Walters, K. J. Gates, channels, and switches: Elements of the proteasome machine. Trends Biochem. Sci. 41, 77-93 (2016).

49. Zhang, N.-Y. Y., Tang, Z. \& Liu, C.-W. W. A-synuclein protofibrils inhibit 26S proteasomemediated protein degradation: Understanding the cytotoxicity of protein protofibrils in neurodegenerative disease pathogenesis. J. Biol. Chem. 283, 20288-20298 (2008).

50. Díaz-Hernández, M. et al. Neuronal induction of the immunoproteasome in Huntington's disease. J. Neurosci. 23, 11653-11661 (2003).

51. Walsh, D. M. \& Selkoe, D. J. Oligomers on the brain: the emerging role of soluble protein aggregates in neurodegeneration. Protein Pept Lett. 3, 213-28 (2004).

52. Stefanis, L. alpha-Synuclein in Parkinson's disease. Cold Spring Harb. Perspect. Med. 2, 1-23 (2012).

53. Arrasate, M., Mitra, S., Schweitzer, E. S., Segal, M. R. \& Finkbeiner, S. Inclusion body formation reduces levels of mutant huntingtin and the risk of neuronal death. Nature 431, 805-810 (2004).

54. Castillo-Carranza, D. L., Guerrero-Muñoz, M. J. \& Kayed, R. Therapeutic approaches against common structural features of toxic oligomers shared by multiple amyloidogenic proteins. Biochem. Pharmacol. 88, 468-478 (2014).

55. Kayed, R. et al. Common structure of soluble amyloid oligomers implies common mechanism of pathogenesis. Science 300, 486-489 (2003).

56. Kayed, R. et al. Fibril specific, conformation dependent antibodies recognize a generic epitope common to amyloid fibrils and fibrillar oligomers that is absent in prefibrillar oligomers. Mol. Neurodegener. 2, 18 (2007).

57. Lesné, S. E. et al. Brain amyloid-beta oligomers in ageing and Alzheimer's disease. Brain 136, 1383-98 (2013).

58. Yang, T., Li, S., Xu, H., Walsh, D. M. \& Selkoe, D. J. Large soluble oligomers of amyloid $\beta$ protein from Alzheimer brain are far less neuroactive than the smaller oligomers to which they dissociate. J. Neurosci. 37, 152-163 (2017).

59. Barghorn, S. et al. Globular amyloid $\beta$-peptide1-42 oligomer - A homogenous and stable neuropathological protein in Alzheimer's disease. J. Neurochem. 95, 834-847 (2005).

60. Bernstein, S. L. et al. Amyloid- $\beta$ protein oligomerization and the importance of tetramers and dodecamers in the aetiology of Alzheimer's disease. Nat. Chem. 1, 326-331 (2009).

61. Lesné, S. et al. A specific amyloid- $\beta$ protein assembly in the brain impairs memory. Nature 440, 352-357 (2006).

62. Ahmed, M. et al. Structural conversion of neurotoxic amyloid-beta(1-42) oligomers to fibrils. Nat. Struct. Mol. Biol. 17, 561-567 (2010).

63. Sandberg, A. et al. Stabilization of neurotoxic Alzheimer amyloid- $\beta$ oligomers by protein engineering. Proc Natl Acad Sci U S A 107, 15595-600 (2010).

64. Cheng, I. H. et al. Accelerating amyloid-beta fibrillization reduces oligomer levels and functional deficits in Alzheimer disease mouse models. J Biol Chem 282, 23818-23828 (2007).

65. Kayed, R., Mcintire, T. M., Milton, S. C. \& Cotman, C. W. Common structure of soluble amyloid oligomers implies common amyloid oligomers implies common mechanism of pathogenesis. Science, 486, (2012). 
66. Köhler, A. et al. The axial channel of the proteasome core particle is gated by the Rpt2 ATPase and controls both substrate entry and product release. Mol. Cell 7, 1143-1152 (2001).

67. Unverdorben, P. et al. Deep classification of a large cryo-EM dataset defines the conformational landscape of the $26 \mathrm{~S}$ proteasome. Proc. Natl. Acad. Sci. U. S. A. 111, 5544-5549 (2014).

68. Śledź, P. et al. Structure of the $26 \mathrm{~S}$ proteasome with ATP-yS bound provides insights into the mechanism of nucleotide-dependent substrate translocation. Proc. Natl. Acad. Sci. U. S. A. 110, 7264-7269 (2013).

69. Whitby, F. G. et al. Structural basis for the activation of $20 \mathrm{~S}$ proteasomes by $11 \mathrm{~S}$ regulators. Nature 408, 115-120 (2000).

70. Russell, S. J., Steger, K. A. \& Johnston, S. A. Subcellular localization, stoichiometry, and protein levels of 26S proteasome subunits in yeast. J Biol Chem. 274, 21943-21952 (1999).

71. Hashimoto, M. et al. Analysis of microdissected human neurons by a sensitive ELISA reveals a correlation between elevated intracellular concentrations of $A \beta 42$ and Alzheimer's disease neuropathology. Acta Neuropathol. 119, 543-54 (2010).

72. Kisselev, A. F., Callard, A. \& Goldberg, A. L. Importance of the different proteolytic sites of the proteasome and the efficacy of inhibitors varies with the protein substrate. $\mathrm{J} \mathrm{Biol} \mathrm{Chem}$ 281, 8582-8590 (2006).

73. Smith, D. M. et al. ATP binding to PAN or the 26S ATPases causes association with the $20 \mathrm{~S}$ proteasome, gate opening, and translocation of unfolded proteins. Mol. Cell 20, 687698 (2005).

74. Asano, S. et al. Proteasomes. A molecular census of $26 \mathrm{~S}$ proteasomes in intact neurons. Science. 347, 439-442 (2015).

75. Luan, B. et al. Structure of an endogenous yeast $26 \mathrm{~S}$ proteasome reveals two major conformational states. Proc. Natl. Acad. Sci. U. S. A. 113, 2642-7 (2016).

76. Matyskiela, M. E., Lander, G. C. \& Martin, A. Conformational switching of the 26S proteasome enables substrate degradation. Nat. Struct. Mol. Biol. 20, 781-8 (2013).

77. Wehmer, M. et al. Structural insights into the functional cycle of the ATPase module of the 26 S proteasome. Proc. Natl. Acad. Sci. 114, 201621129 (2017).

78. Choi, W. H. et al. Open-gate mutants of the mammalian proteasome show enhanced ubiquitin-conjugate degradation. Nat. Commun. 7, 10963 (2016).

79. Unno, M. et al. The structure of the mammalian $20 \mathrm{~S}$ proteasome at 2.75 angstrom resolution. Structure 10,609-618 (2002).

80. Besche, H. C. \& Goldberg, A. L. Affinity purification of mammalian 265 proteasomes using an ubiquitin-like domain. Methods Mol. Biol. 832, 423-432 (2012).

81. Yu, Y. et al. Interactions of PAN's C-termini with archaeal $20 S$ proteasome and implications for the eukaryotic proteasome-ATPase interactions. EMBO J. 29, 692-702 (2010).

82. Le Feuvre, A. Y., Dantas-Barbosa, C., Baldin, V. \& Coux, O. High yield bacterial expression and purification of active recombinant PA28 $\alpha \beta$ complex. Protein Expr. Purif. 64, 219-24 (2009).

83. Kisselev, A. F., Kaganovich, D. \& Goldberg, A. L. Binding of hydrophobic peptides to several non-catalytic sites promotes peptide hydrolysis by all active sites of $20 \mathrm{~S}$ proteasomes. J Biol Chem 277, 22260-22270 (2002).

84. Martinez-Fonts, K. \& Matouschek, A. A rapid and versatile method for generating proteins with defined ubiquitin chains. Biochemistry 55, 1898-1908 (2016).

85. Jan, A., Hartley, D. M. \& Lashuel, H. a. Preparation and characterization of toxic $A \beta$ aggregates for structural and functional studies in Alzheimer's disease research. Nat. Protoc. 5, 1186-209 (2010).

86. Lotz, G. P. et al. Hsp70 and Hsp40 functionally interact with soluble mutant huntingtin 
oligomers in a classic ATP-dependent reaction cycle. J. Biol. Chem. 285, 38183-38193 (2010). 


\title{
Study 2: Mechanisms of proteasome gate activation
}

\author{
Tiffany A. Thibaudeau ${ }^{1}$ and David M. Smith ${ }^{1 *}$ \\ Manuscript in preparation
}

${ }^{1}$ Program in Biochemistry and Molecular Biology, Department of Biochemistry, West Virginia University, Morgantown, WV

* Corresponding Author: David M. Smith, West Virginia University Department of Biochemistry, P.O. Box 9142, Morgantown WV, 26506. Email: dmsmith@hsc.wvu.edu

Keywords. Proteasome, proteasome gate activators, $\mathrm{HbYX}$ motif

Financial support: NIH-R01GM107129 to D.M.S.

The content is solely the responsibility of the authors and does not necessarily represent the official views of the $\mathrm{NIH}$. 


\section{Abstract}

Proteostasis is maintained by several systems in the cell including the ubiquitin-proteasome system (UPS), chaperones, chaperone-mediated autophagy, and macroautophagy. The UPS is the principle route for the degradation of intracellular misfolded, damaged, or unneeded proteins. At the center of the UPS is the proteasome, a large and complex molecular machine containing a multicatalytic protease complex. When the efficiency of this proteostasis systems is perturbed, misfolded and damaged protein aggregates can accumulate to toxic levels and cause neuronal dysfunction, which may underlie many neurodegenerative diseases. The $20 \mathrm{~S}$ proteasome substrate-entry gate plays a critical role in proteasome function and in cellular proteostasis and the molecular mechanisms of gating remain poorly understood. In this study we develop a novel compound that promotes proteasome gate opening to increase the degradation rate of peptides and proteins. These results provide a new tool for investigating proteasome function and provide proof -of-concept development of gate-activating compounds. 


\section{Introduction}

The ubiquitin-proteasome system (UPS) is responsible for more regulated protein degradation in eukaryotes and regulates myriad cellular processes, such as the cell cycle, apoptosis, the immune response, inflammation, and the response to proteotoxic stress ${ }^{1,2}$. Proteins selected for degradation are typically modified by the covalent attachment of ubiquitin chains, which targets it to the $26 \mathrm{~S}$ proteasomes for degradation ${ }^{2,3}$. The $26 \mathrm{~S}$ proteasome is a complex and highly regulated 2.5 MDa peptidase complex, comprised of a barrel-shaped proteolytic 20S core particle with a 19S ATPase regulatory particle capped on one or both barrel ends ${ }^{4}$. Ubiquitinated proteins are degraded in a multistep process; the 195 regulatory particle binds ubiquitinated substrates, opens a substrate entry gate in the $20 \mathrm{~S}^{5,6}$, and unfolds its substrates by linearly translocating them into the $20 \mathrm{~S}$ catalytic chamber where they are degraded to peptides ${ }^{4,7}$.

Compartmentalized proteases protect the cellular milieu from proteolytic activity and retain the capacity to degrade specific proteins in a regulated manner. Eukaryotic $20 \mathrm{~S}$ proteasomes have four stacked heteroheptameric rings arranged in an $\alpha_{1-7-} \beta_{1-7-} \beta_{1-7-} \alpha_{1-7}$ fashion ${ }^{8}$. The two $\beta$ rings form a catalytic chamber with three different peptidase activities (chymotrypsin-like site ( $\beta 5)$ preferentially cleaves after hydrophobic residues, the trypsin-like site ( $\beta 2)$ preferentially cleaves after basic residues, and the caspase-like site $(\beta 1)$ preferentially cleaves after acidic residues $\left.{ }^{4,9}\right)$. The a subunits' amino(N)-termini extend over the 13 Angstrom central pore and form a gate, which occludes access to the proteolytic sites located inside ${ }^{10,11}$. The $20 \mathrm{~S}$ proteasome substrate-entry gate plays a critical role in proteasome function and in cellular proteostasis. The $\alpha 3 \mathrm{~N}$-terminus is the lynchpin and forms salt bridges with neighboring $\mathrm{N}$-termini to stabilize the closed gate confirmation $^{12}$. Deletion of the first eight $\alpha 3$ residues $\alpha 3 \Delta N$ ( $\left.\alpha 3 \Delta N-20 S\right)$ sufficiently destabilizes the closed-gate conformation and accelerates the entry and degradation of peptides ${ }^{12}$. Wild type $20 S$ proteasome activity is similarly accelerated when bound to a proteasome activator (e.g. 19S/PA700, 11S/PA28, BIm10/PA200) ${ }^{13}$. 
Proteasome activators bind to one or both ends of the $20 \mathrm{~S}$ core particle by inserting one or more C-termini into pockets between adjacent 20S $\alpha$ subunits (intersubunit pockets). Proteasome activators stabilize the conserved proline reverse turn (at the base of the N-termini gate) in the "open" conformation, using two different mechanisms. Most proteasome activators' C-termini (19S/PA700, BIm10/PA200, PAN) contain a hydrophobic-tyrosine-X (HbYX) motif (Figure 1A,B). The penultimate tyrosine forms a hydrogen bond with the proline reverse turn to stabilize the open conformation and trigger gate opening ${ }^{14,15}$. In contrast, the C-termini of 11S/PA28/PA26 family of proteasome activators do not have a HbYX motif, instead they use an internal activation loop to contact and reposition the proline reverse turn ${ }^{16}$.

Recent structural studies (cryo-EM) of the eukaryotic $26 \mathrm{~S}$ proteasome highlight the complexities of $26 \mathrm{~S}$ gate opening. Several studies suggest that the conserved $\mathrm{HbYX}$ motif of three $19 \mathrm{~S}$ subunits (Rpt2,Rpt3,Rpt5) stably insert into the intersubunit pockets without causing full gate opening $^{17-20}$. In contrast, functional studies have shown that 195 binding to the $20 \mathrm{~S}$ stimulates gateopening in the $20 \mathrm{~S}$ proteasome $\mathrm{e}^{6,21}$, in a $\mathrm{HbYX}$ motif dependent manner ${ }^{22}$, and binding of the $\mathrm{HbYX}$ motif by itself (as a hepta-peptide) is also sufficient to allosterically induce conformational changes in the $\alpha$-subunits that cause gate opening ${ }^{14,15}$. Hence, the molecular mechanism of gating by the $\mathrm{RP}$ and how the gate dynamics change when a the $20 \mathrm{~S}$ associates with an activator remains poorly understood. Drugs or genetic mutations that increase proteasome activity restore proteostasis in cellular models of neurodegenerative disease ${ }^{23-25}$. Therefore, there is a need for a better understanding of the $20 \mathrm{~S}$ gate mechanism.

In this study we design a small proteasome activator (ZYA) based on the molecular interactions of the conserved $\mathrm{C}$-terminal $\mathrm{HbYX}$ motif in proteasome activators. We show that ZYA activates proteasome function by opening the $20 \mathrm{~S}$ gate to increase degradation of both peptide and protein substrates. ZYA has potential application as a research tool to study proteasome function in vitro 
and in vivo, and we demonstrate the feasibility of designing drug-like molecules to activate proteasome function to treat patients with neurodegenerative diseases.

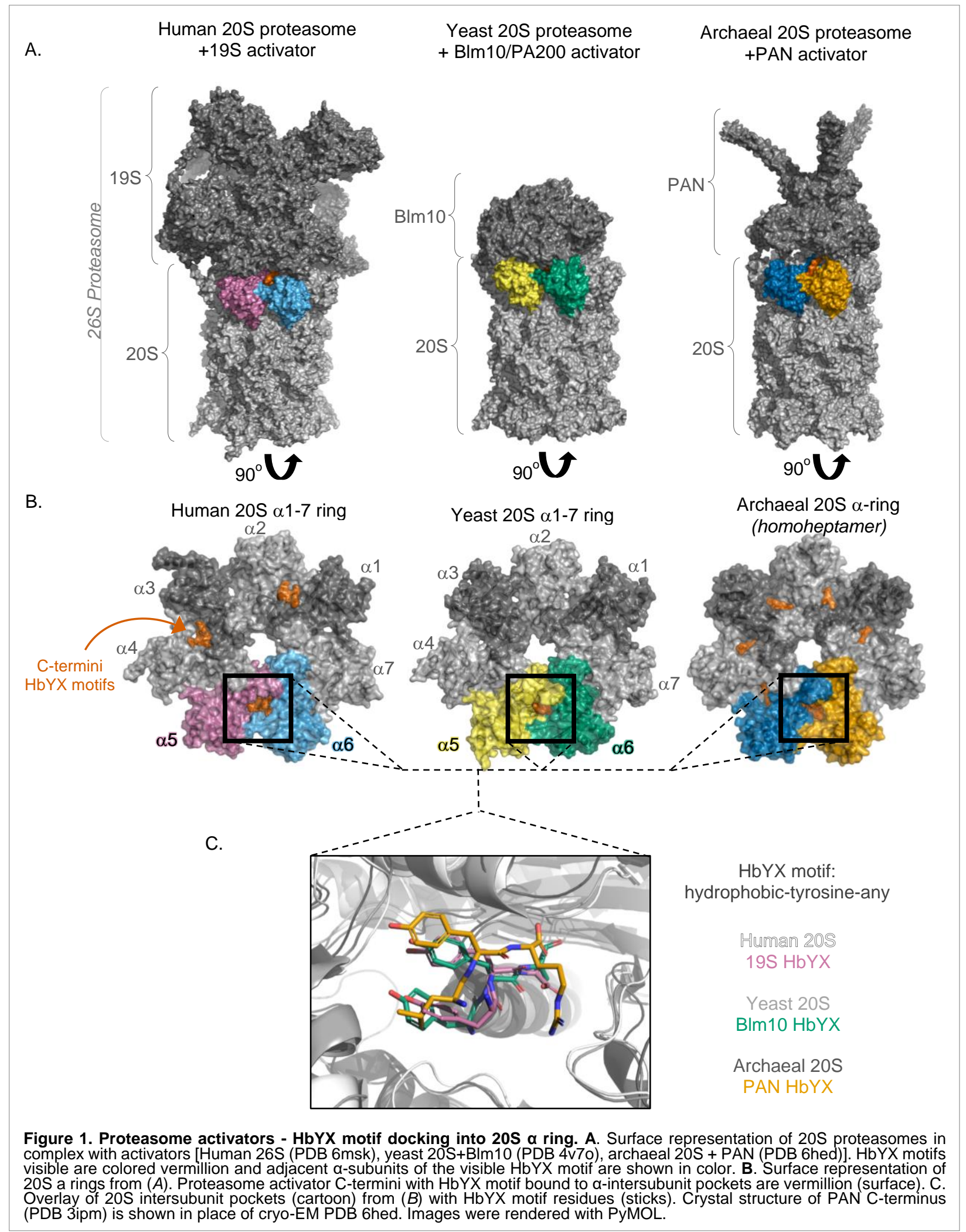




\section{Results and Discussion}

The conserved HbYX motif is found in proteasome activators from archaea to humans. We aligned activator-bound intersubunit $\alpha$ pockets from human (PDB $6 \mathrm{msk}^{17}$, cryo-EM), yeast (PDB $\left.4 \mathrm{v}^{2} \mathrm{o}^{26}\right)$, and archaeal proteasomes (PDB 6hed ${ }^{27}$, cryo-EM) (Figure 1C). As expected, the C-terminal $\mathrm{HbYX}$ motif bound to the intersubunit pockets in similar orientations, with the C-terminal carboxylic acid directed towards the conserved pocket lysine (which is required for 20S proteasome-activator complex formation ${ }^{14}$ ) and the penultimate tyrosine hydroxyl group oriented toward the proline reverse turn (located at the gate base). These two interactions are critical for HbYX motif binding to and activating the proteasome gate ${ }^{14,15,28}$. Our aim was to further characterize the HbYX binding pocket and apply this information to the design of small synthetic gate activating molecules.

The intersubunit pockets contain many conserved residues, some of which are known to be important for gate activation (e.g. Pro17, Lys66) ${ }^{28,29}$. We investigated the effect of mutations to three conserved residues positioned near the bound $\mathrm{HbYX}$ motif (Figure 2A). For mutational analysis, we used the archaeal (Thermoplasma acidophilum) $20 \mathrm{~S}$ because it is a homoheptamer, amenable to mutational studies, and easily expressed in E.coli. Consistent with previously published results ${ }^{14}$, Lys66Ala mutation prevented PAN $^{30}$ (the archaeal homologue of the 19S) activation of the $20 \mathrm{~S}$ (Figure 2B). Lys33 is located at the base of the a-helix leading toward the proline reverse turn. When K33 is mutated to a glycine, 20S activity was less than half that of the wild type and was not stimulated by PAN. This could be due to a disruption of the helices by inserting the secondary structure-destabilizing glycine, or by loss of the $\mathrm{K} 33 \varepsilon$-amine bonding interactions. Leucine 81 forms the bottom of the HbYX pocket directly underneath the HbYX tyrosine. Loss of this hydrophobic residue (by mutation to alanine or glycine) prevents PAN mediated proteasome activation (Figure $2 \mathrm{~B}$ ), suggesting this residue is important for stabilizing $\mathrm{HbYX}$ motif binding. We noted the proximity of Leu81 to the reverse turn backbone (Gly19). Considering the importance 
of $\mathrm{HbYX}$ tyrosine hydrogen bonding with the backbone, we asked if a tyrosine substitution at this position would mimic the effect of a bound HbYX motif. Surprisingly, proteasomes with the Leu81Tyr mutation ( $\alpha^{\mathrm{L} 81 Y} T 20 S$ ) had over three-fold greater basal activity compared to wild type (Figure 2C), suggesting this mutation stabilizes the open-gate conformation. In agreement with this, addition of PAN to $a^{\mathrm{L} 81 Y} T 20$ s proteasomes only resulted in $30 \%$ increased activation, whereas an equal amount of PAN increased wild-type T20S activity greater than five-fold (Figure $2 \mathrm{C})$. These data show that $20 \mathrm{~S}$ gate activation can be achieved by small scale alteration of the intersubunit pocket.

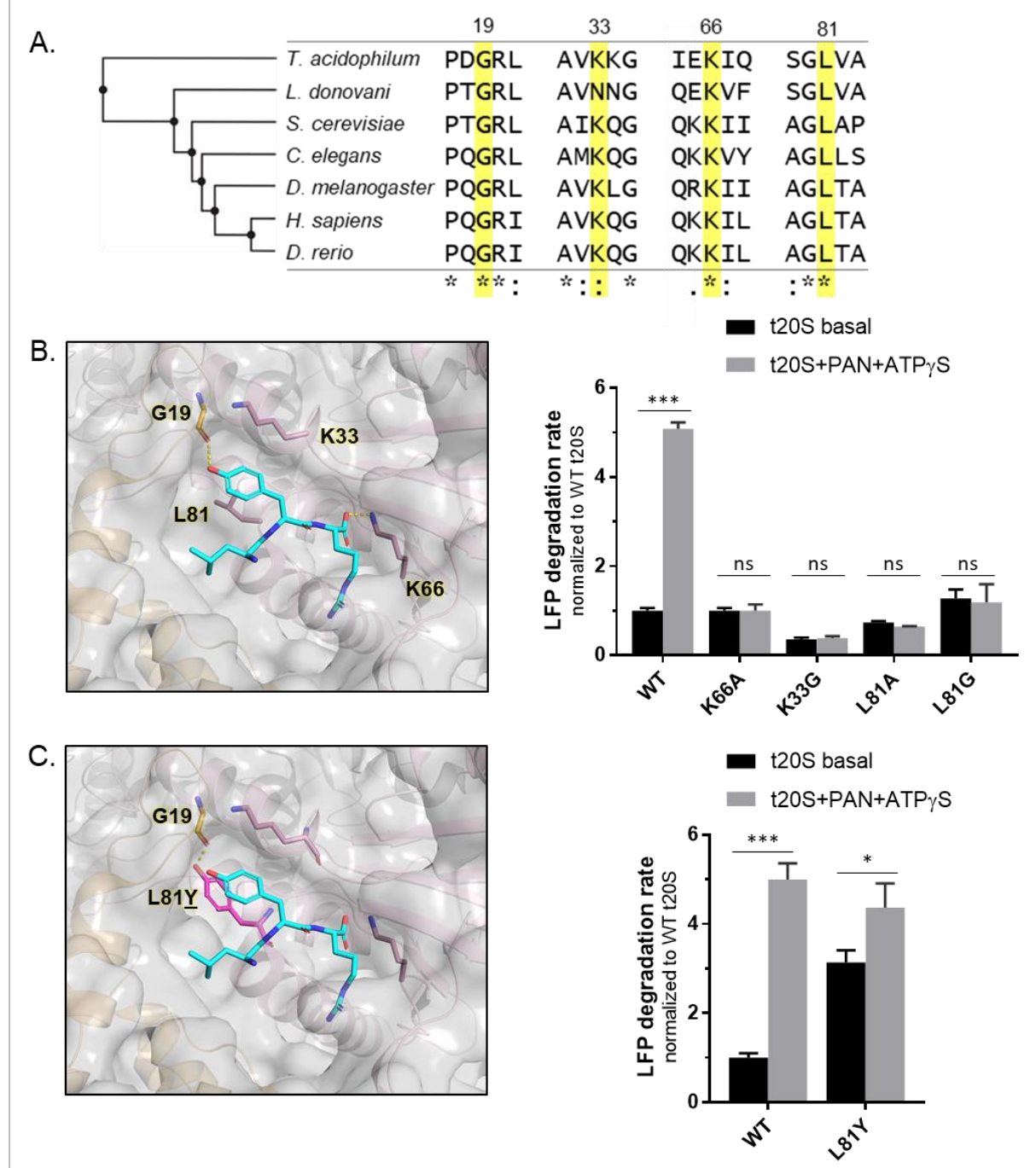

Figure 2. Highly conserved $\alpha$-intersubunit pocket residues are re-
quired for PAN-HbYX gate activation. A. Multiple sequences alignment (20S a6 protein) generated with CLUSTAL OMEGA (1.2.4). B. Left, conserved residues interacting with the bound HbYX motif (sticks) are shown in the t20S intersubunit binding pocket (PDB 3ipm). The PAN HbYX motif (LYR) is shown in cyan (stick). Right, t20S proteasome $(0.2 \mu \mathrm{g}$ of wild-type or K66/K33/L81 mutants) and fluorogenic octapeptide (LFP) are incubated
with or without PAN and ATPYS. The stimulation of gate opening was measured by the increase of LFP hydrolysis (rfu/min) over wild-type $20 \Omega$ without any activator. C. Left, same as $(B)$, with L81 mutated to tyrosine (magenta stick). Right, experiments with t20S proteasome $(0.2$ $\mu \mathrm{g}$ of wild-type or L81Y mutant) performed same as in $(B)$. Images in $(B)$ and $(C)$ were rendered with PyMOL. The data are representative of three or more independent experiments performed in triplicate. Error bars represent \pm standard deviation. Panels $B$ and $C$ : ${ }^{* * *} p<0.0001 ;$ ns $p>0.05$. (students $t$ test), P-value compared to t20s basal activity. 
Previous studies showed that short (3-6 amino acid) peptides with a C-terminal HbYX motif (corresponding to the PAN C-terminus) did not activate the $20 \mathrm{~S}$ gate ${ }^{14,15}$. This contrasts with our Leu81Tyr mutation data, from which we hypothesized small $\mathrm{HbYX}$ motif peptides can activate the gate. The 3-8 amino acid peptides used in Smith et al. $(2007)^{14}$ were synthesized with unmodified $\mathrm{N}$-termini, and this positive charge may prevent shorter $\mathrm{HbYX}$ peptides from binding in the intersubunit pocket. To test this hypothesis, we synthesized $\mathrm{N}$-terminal acetylated peptides corresponding to the PAN C-terminus from three to eight residues in length (CT3-8). In contrast to previous results ${ }^{14}$, PAN CT peptides shorter than seven residues stabilized the open $20 \mathrm{~S}$ gate (Figure 3A). Based on the successful gate-activation of PAN CT3 (Ac-LYR), we designed a small, novel dipeptide to mimic the HbYX-motif. We substituted an alanine in place of the bulky arginine residue to decrease the dipeptide size, as this substitution did not alter ability of PAN-CT8 peptide to bind $\alpha$ intersubunit pockets and open the gate ${ }^{14,15}$ in previous studies. Capping the tyrosine N-

Figure 3. HbYX of peptides of different lengths to stimulate gate opening A. Peptides $(200 \mu \mathrm{M})$ were incubated with $0.2 \mu \mathrm{g}$ t20s proteasomes and $10 \mu \mathrm{M}$ FP substrate. A correction was made because the added peptides also compete with LFP at the active sites. To determine the actual percentage stimulation of LFP hydrolysis due to gate opening, the ability of the various peptides to in hibit LFP hydrolysis by the gateless $(\Delta \alpha 2-12)$ 20S proteasome was also measured, and the values were used to normalize data on LFP hydrolysis by wild-type $20 S$ proteasomes. Without normalization, the three- to five-residue peptides still stimulated 20S proteasomes 2- to 3-fold. B. $0.2 \mu \mathrm{g}$ t20S proteasomes incubated with $10 \mu \mathrm{M}$ LFP substrate and DMSO or 200 $\mu M$ ZYA. LFP degradation rate (rfu/min) is normalized to DMSO. The data are representative of three or more independent experiments performed in triplicate. Error pars represent \pm standard deviation. $\quad{ }^{* * *} p<0.0001$; ${ }^{* *} \mathrm{p}<0.01$ (student $t$ test), $\mathrm{P}$ value compared to DMSO.
A.

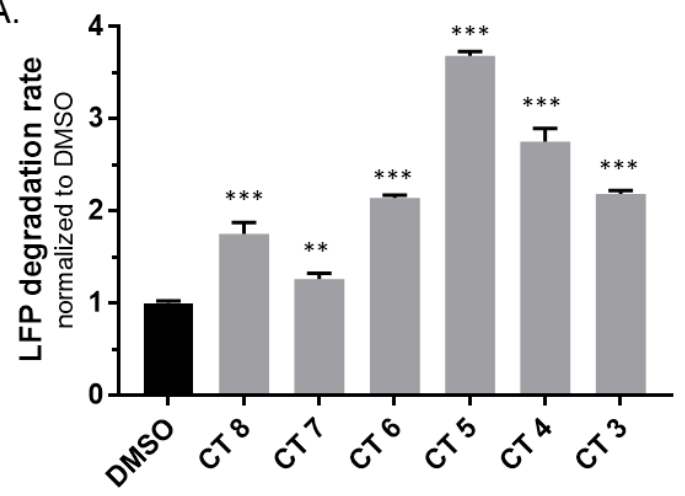

PAN C-terminus acetylated peptides $(200 \mu \mathrm{M})$

CT8 Ac-AHLDVLYR

CT7 AC-HLDVLYR

CT6 AC-LDVLYR

CT5 AC-DVLYR

CT4 Ac-VLYR

CT3 AC-LYR

B.

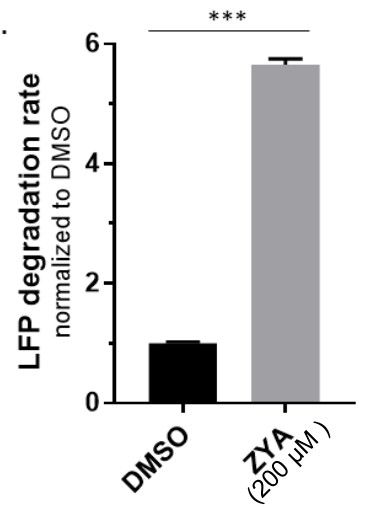<smiles>CC(NC(=O)C(Cc1ccc(O)cc1)NC(=O)OCc1ccccc1)C(=O)[O-]</smiles>

ZYA (Carboxybenzyl-Tyrosine-Alanine) 
terminus with a carboxybenzyl group allowed the synthesis of a dipeptide that retained a hydrophobic group preceding the tyrosine. We tested ZYA with T20S proteasomes and observed almost 6-fold activation (Figure 3B).

Our next step was to test ZYA with the more complex heteroheptameric 20S proteasomes found in eukaryotes. Consistent with our previous results, ZYA activated the mammalian 20 S proteasome (Figure 4A). When the charged carboxylic acid was modified with $\mathrm{NOH}_{2}$ group, (i.e. no longer carried a negative charge) ZYA-[NOH$]$ failed to activate the proteasome (Figure 4A). Since the charged C-terminus is critical for $\mathrm{HbYX}$ mediate gate activation, we evaluated the effect of adding additional negative charges to the HbYX-motif dipeptide (ZYD, ZYE), a backbone torsion constraint (ZYP), and a polar group (ZYQ). None of these "X" modifications activated the mammalian $20 \mathrm{~S}$ proteasome (Figure 4A).

Since hydrogen bonding between the HbYX hydroxyl group and G19 in the proline reverse turn is important for gate activation by proteasome activators and their C-terminal peptides ${ }^{31}$, we determined the effect of modifying the ZYA tyrosine. The tripeptide Ac-YFA partially activated the $20 \mathrm{~S}$ proteasome gate (Figure 4B). Although 
A.

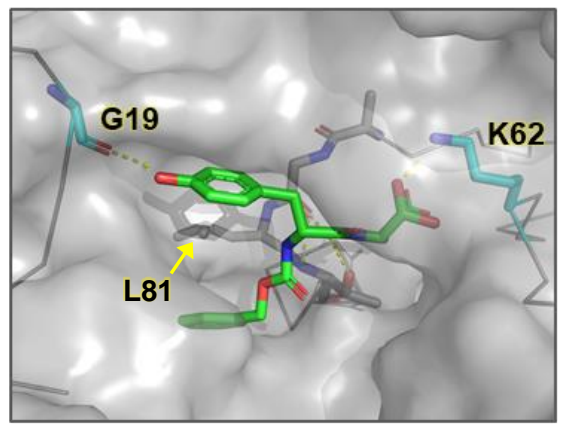

D.
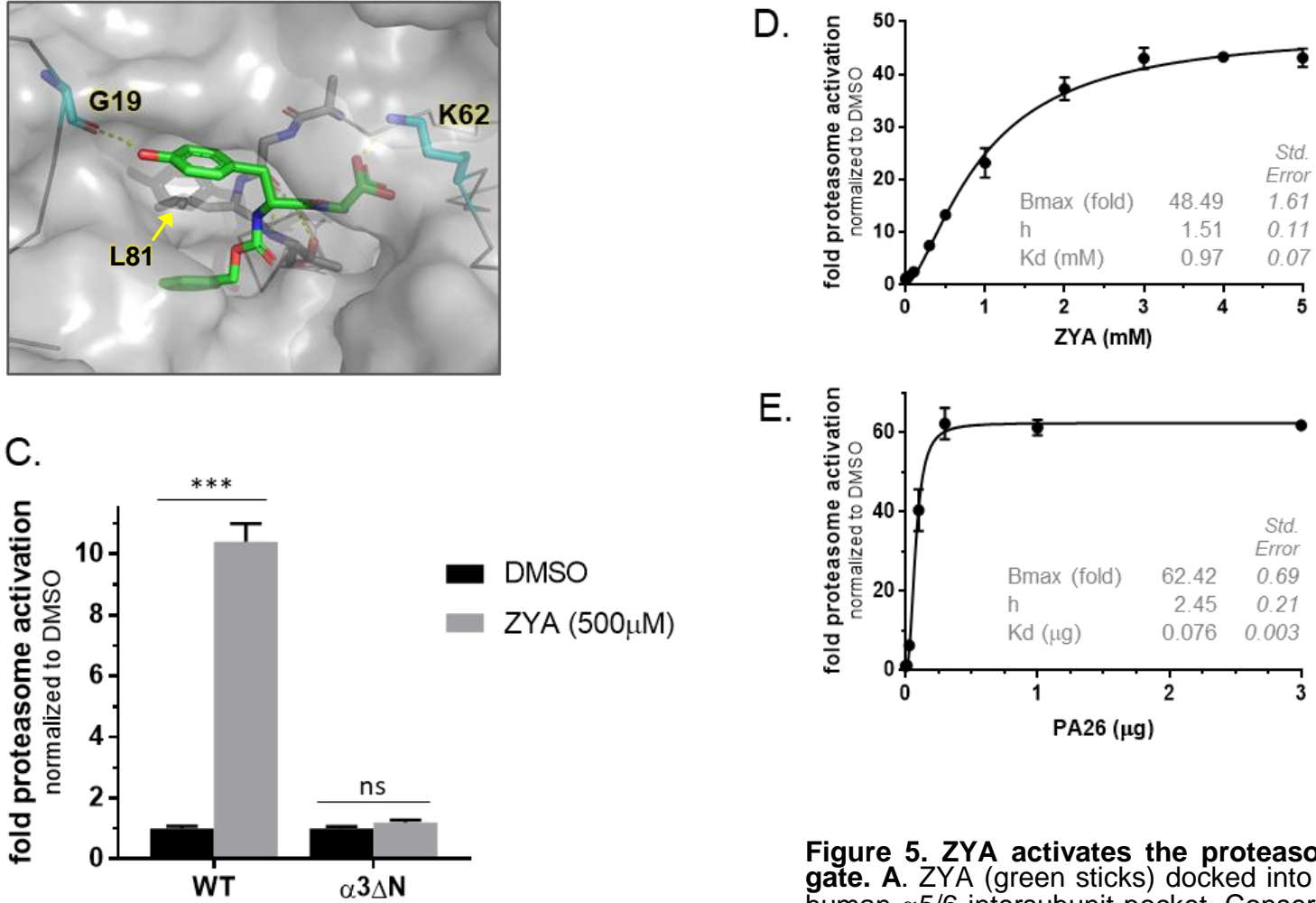

DMSO

ZYA $(500 \mu M)$

E.

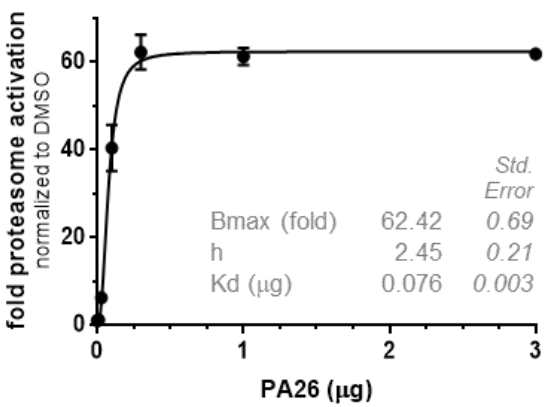

Figure 5. ZYA activates the proteasome gate. A. ZYA (green sticks) docked into the human $\alpha 5 / 6$ intersubunit pocket. Conserved G19 and K62 (corresponding to K66 in T. acidophilum 20S) shown as cyan sticks. Yellow arrow points to L81. Image was rendered in PyMOL. B. Mammalian 20S proteasomes $(0.5 \mathrm{nM})$ were incubated with $1 \mathrm{mM}$ ZYA and $100 \mu \mathrm{M}$ of the indicated peptide substrate. Proteasome activity (linear rate of substrateamc hydrolysis) is normalized to DMSO controls. C. Yeast wild-type and gate mutan $\alpha 3 \Delta \mathrm{N} 20 \mathrm{~S}$ proteasomes incubated with 500 $\mu M$ ZYA. Proteasome activity (linear rate of nLPnLD-amc hydrolysis) is normalized to DMSO controls. D. Mammalian 20S proteasomes $(0.5 \mathrm{nM})$ were incubated with ZYA at the indicated concentrations. Proteasome activity (linear rate of substrate-amc hydrolysis) is normalized to DMSO control. The data are representative of three or more independent experiments performed in triplicate. Error bars represent \pm standard deviation. Panels $B$ and $C:{ }^{* * * *} p<0.0001 ;{ }^{* \star *} p<0.001$; ns $\mathrm{p}>0.05$. (student $t$ test).

not a strict C-terminal $\mathrm{HbYX}$ motif, this peptide retains a tyrosine, and it is worth noting that some eukaryotic proteasome activators have a -YYA HbYX motif (i.e. human Rpt5, yeast BIm10). The ability of Ac-YFA to activate the proteasome gate suggests the intersubunit binding pocket is "flexible" as to what peptides it can successfully bind. Modifications disrupting hydrogen bond 
donating availability of the ZYA tyrosine hydroxyl group (Z-pY-A and Z-[3,nitroTyr]-A) do not activate the proteasome (Figure 4B). Interestingly, when 4-aminophenylalanine is substituted for the tyrosine (Z-[4,aminoPhe]-A) the proteasome is activated like ZYA (Figure 4B). Unlike phosphotyrosine and 3-nitrotyrosine, 4-aminophenylalanine's mass and polar surface area is like tyrosine's, and it has the same hydrogen bond availability. These results are consistent with the computational docking model of ZYA in the human a 5/6 intersubunit pocket (Figure 5A).

Our next aim was to use biochemical methods to confirm ZYA activates the 20S proteasome by stabilizing the open gate conformation. Eukaryotic proteasomes have three different types of active sites, and stabilization of the open gate conformation increases activity from all three sites. We tested the effect of ZYA on proteasome hydrolysis of three substrates preferentially cleaved by different sites (LLVY-amc, $\beta 5$; nLPnLD-amc, $\beta 1$; LRR-amc, $\beta 2$ ). As expected, ZYA increased the hydrolysis rate of all three substrate peptides (Figure 5B). To confirm ZYA indeed influences the $20 \mathrm{~S}$ gate, we performed activity assays using yeast $20 \mathrm{~S}$ proteasomes with a gate mutation $(\alpha 3 \Delta N)$ that prevents the gate from fully closing ${ }^{10}$. The $\alpha 3 \Delta N$ proteasome gates allow substrate peptides unrestricted access to the proteolytic core. While ZYA activates wild-type yeast proteasome activity, it does not increase $\alpha 3 \Delta \mathrm{N}$ proteasome activity (Figure $5 \mathrm{C}$ ). We titrated ZYA with $20 S$ proteasomes to determine maximum activation and affinity. Although ZYA can stimulate proteasome activity over 40-fold, it has poor affinity (Figure 5D). Interestingly, ZYA has hill coefficient of 1.5 indicative of positive cooperative binding, which matches recently proposed cryo-EM structural models for 19S RPT C-termini opening the gate ${ }^{17-20}$.

While we have demonstrated ZYA activates peptide hydrolysis via opening its gate, changes in peptidase activity do not necessarily reflect changes in the proteasome's capacity to degrade proteins. To evaluate the efficacy of ZYA to stimulate protein degradation, we used SDS-PAGE to measure the effect of ZYA has on proteasome degradation of tau23 (a truncated tau protein) and casein, which are protein substrates of the 20S. ZYA significantly increased the degradation 


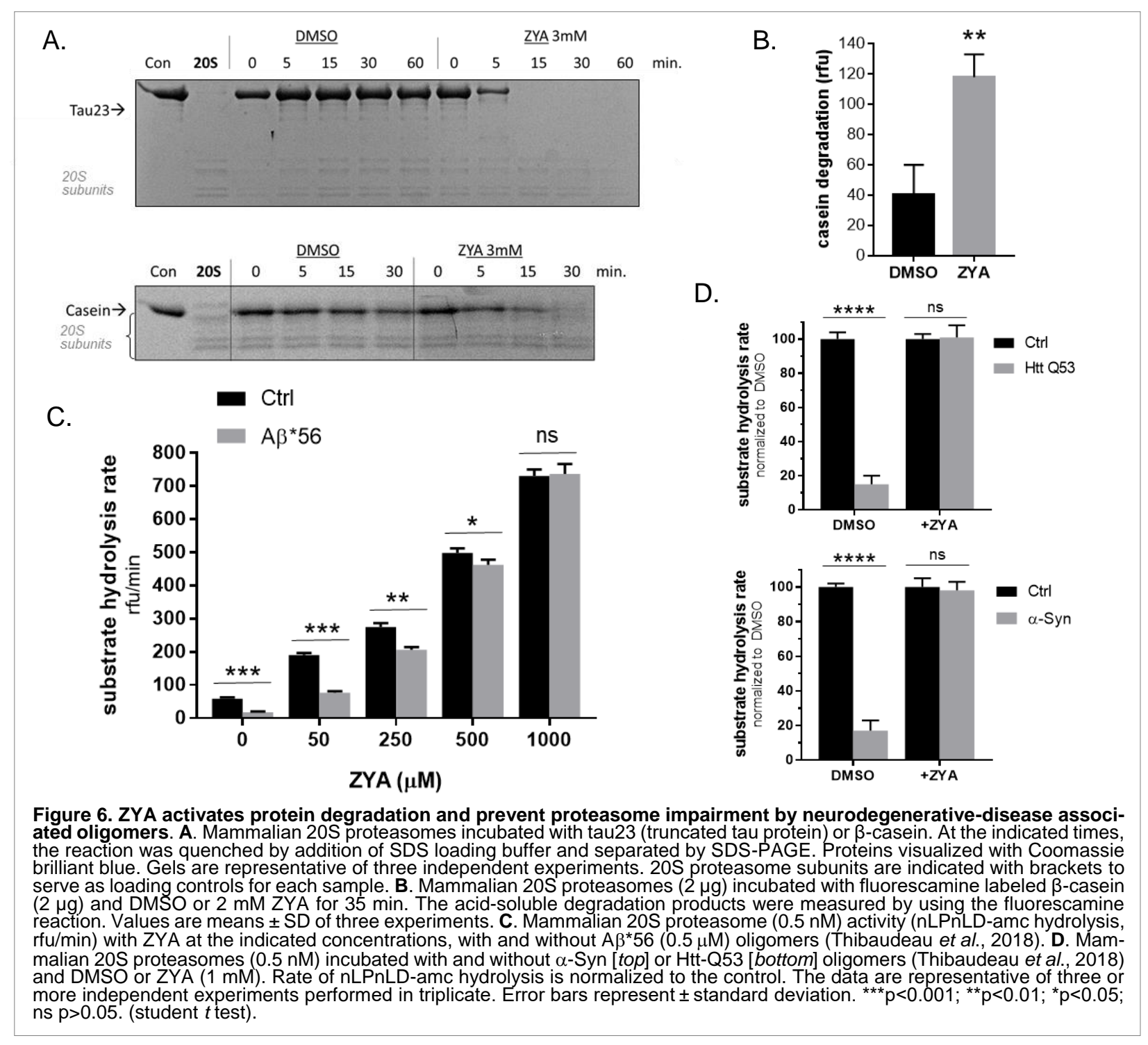

of both proteins (Figure 6A). In support of these results, we measured $20 \mathrm{~S}$ proteasome degradation of $\beta$-casein (Figure 6B). In agreement with the gel-based protein degradation assay, ZYA significantly increased proteasome degradation $\beta$-casein.

Thus far we have demonstrated ZYA increases peptide and protein degradation by increasing 20 s proteasome gate-opening. We recently published a study demonstrating protein oligomers associated with neurodegenerative diseases (A $\beta$, $\alpha$-synuclein, and huntingtin with a Q53 expansion) impair proteasome function by stabilizing the closed gate conformation 2019. This study 
showed an 8-amino acid HbYX peptide derived from the Rpt5 C-terminus partially restored proteasome activity in the presence of these oligomers. Remarkably, when proteasome activity is measured in the presence of oligomers, the addition of ZYA completely restores proteasome function (Figure 6C). This is an important demonstration for the potential small molecules to restore activity in conditions of proteasome impairment.

\section{Concluding Remarks}

The proteasome is a highly regulated multi-subunit complex and possesses multiple drug targets to modulate its degradation capacity. The first compounds to target the proteasome inhibited the proteolytic sites in the $20 \mathrm{~S}$ core particle. In fact, early understanding of the importance of the proteasome for biological functions and processes rapidly advanced with the introduction of the first proteasome inhibitors ${ }^{32}$. More recently, compounds targeting a 19S-associated deubiquitinase (USP14) have been developed. Treatment of cultured cells with these inhibitors can increase the rate of degradation of a subset of proteasome substrates ${ }^{33}$, and accelerate the rate of degradation of wild-type and pathological tau mutants ${ }^{34}$. In contrast, development of compounds to stimulate gate-opening have received little attention. Recent structural studies (cryoEM) of the eukaryotic 26S proteasome highlight the complexities of 26S gate opening. Considering the importance of the proteasome gate in regulating protein degradation, gate-activating compounds (such as ZYA) have great potential as research tools to probe proteasome function in vitro and in vivo.

Decreased proteasome function has been reported in a broad array of chronic neurodegenerative diseases $^{35}$, and proteasomal upregulation accelerates the clearance of pathogenic proteins. Studies suggest the elevation of proteasomal activity is tolerable to cells and may be beneficial to prevent the accumulation of protein aggregates. For example, Cells treated with exogenous pro- 
teasomes are more efficient in degrading overexpressed human tau than endogenous proteasomal substrates, resulting in decreased levels of tau aggregates ${ }^{36}$. Similar results are seen in cells expressing the $\alpha 3 \Delta \mathrm{N}$ open gate mutant proteasome ${ }^{37}$. Our study offers proof of concept for the design of small molecules that open the proteasome gate. 


\section{Acknowledgements}

We thank the members of the Smith lab for helpful and valuable discussions.

\section{Author Contributions}

T.A.T. and D.M.S designed and performed experiments, analyzed data, and wrote the manuscript.

\section{Competing financial interests}

The authors declare no competing financial interests. 


\section{Materials and Methods}

\section{Proteins and peptides}

T.acidophilum wild type $20 \mathrm{~S}$ and $\Delta \mathrm{a}^{2-12} 20 \mathrm{~S}$ proteasomes were purified as described ${ }^{30}$. All $20 \mathrm{~S}$ mutants were generated by overlapping PCR site-directed mutagenesis. The plasmid for M.jannaschii PAN(M74A), kindly provided by Dr. Peter Zwick| ${ }^{30}$, lacked a His Tag and was purified as described $^{38}$, but Tris buffers were made at $50 \mathrm{mM}$ instead of $20 \mathrm{mM}$. Mammalian 20S proteasomes were isolated from bovine liver as described ${ }^{39}$. Wild type and mutant $\alpha 3 \Delta \mathrm{N}$ yeast $20 \mathrm{~S}$ proteasomes were expressed and purified by anion-exchange chromatography as described $^{40}$. Fluorogenic substrate peptides were purchased from BostonBiochem (suc-LLVY-

amc) and EZBiolabs (ac-nLPnLD-amc, ac-RLR-amc, LFP (Mca-AKVYPYPME-Dpa(Dnp)-amide)), PAN CT peptides, ZYA, and ZYA derivatives were purchased from Abclonal. Oligomers of $A \beta^{\star} 56$, $\alpha$-synuclein, and huntingtin-Q53 were prepared as described ${ }^{41}$. All oligomers used were recognized by the $\alpha$-oligomer antibody, $\mathrm{A} 11^{42}$. Protein concentrations were determined by Bradford assay (Thermo Scientific).

\section{Enzyme assays}

To measure peptide hydrolysis, fluorogenic peptides in DMSO were used at a final concentration of $100 \mu \mathrm{M}$ for Suc-LLVY-amc and Ac-nLPnLD-amc, and $10 \mu \mathrm{M}$ for LFP and Boc-RLR-amc, in 50 $\mathrm{mM}$ Tris ( $\mathrm{pH}$ 7.5), $1 \mathrm{mM}$ DTT. For archaeal 20S, LFP peptides were added to the buffer at $45^{\circ} \mathrm{C}$, and where indicated, $1 \mu \mathrm{g}$ of PAN and $10 \mu \mathrm{M}$ ATPYS (+5 $\left.\mathrm{mM} \mathrm{MgCl}_{2}\right)$ was added to the 0.1 $\mathrm{ml}$ of reaction buffer (sufficient to saturate the $20 \mathrm{~S}$ particles) ${ }^{43}$. Assays with mammalian $(0.5 \mathrm{nM}$ and $1 \mathrm{nM}$ as indicated), yeast wild-type (2 $\mathrm{nM})$, and yeast $\alpha 3 \Delta \mathrm{N}(0.2 \mathrm{nM})$ proteasomes were performed at $37^{\circ} \mathrm{C}$. Assays with $A \beta$, $\alpha$-synuclein, and huntingtin-Q53 oligomers were performed as described ${ }^{41}$. 


\section{Degradation of proteins}

Tau23 or $\beta$-casein were incubated with mammalian $20 \mathrm{~S}$ proteasomes for the indicated time at $37^{\circ} \mathrm{C}$. Reactions were quenched by addition of LDS sample buffer (Invitrogen). Proteins were separated by SDS-PAGE using NuPAGE ${ }^{\mathrm{TM}} 4-12 \%$ Bis-Tris protein gels (Invitrogen) and visualized with coomassie brilliant blue. The degradation products from $\beta$-casein were measured by using the fluorescamine reaction, as described previously ${ }^{43}$.

\section{Statistical analysis}

Data were analyzed using an unpaired Student's t-test (Prism). For all statistical analyses, a value of $p<0.05$ was considered significant. 


\section{References}

1. Tomko, R. J. \& Hochstrasser, M. Molecular Architecture and Assembly of the Eukaryotic Proteasome. Annu. Rev. Biochem. 82, 415-445 (2013).

2. Ciechanover, A. Intracellular protein degradation: from a vague idea through the lysosome and the ubiquitin-proteasome system and onto human diseases and drug targeting. Bioorganic Med. Chem. 21, 3400-3410 (2017).

3. Kwon, Y. T. \& Ciechanover, A. The Ubiquitin Code in the Ubiquitin-Proteasome System and Autophagy. Trends Biochem. Sci. 42, 873-886 (2017).

4. Voges, D., Zwickl, P. \& Baumeister, W. The $26 \mathrm{~S}$ proteasome: A molecular machine designed for controlled proteolysis. Annu. Rev. Biochem. 68, 1015-1068 (1999).

5. DeMartino, G. N. et al. Identification, purification, and characterization of a PA700dependent activator of the proteasome. J. Biol. Chem. 271, 3112-3118 (1996).

6. Adams, G. M., Crotchett, B., Slaughter, C. A., DeMartino, G. N. \& Gogol, E. P. Formation of proteasome-PA700 complexes directly correlates with activation of peptidase activity. Biochemistry 37, 12927-12932 (1998).

7. DeMartino, G. N. \& Slaughter, C. A. The proteasome, a novel protease regulated by multiple mechanisms. J Biol Chem 274, 22123-22126 (1999).

8. Groll, M. et al. Structure of $20 \mathrm{~S}$ proteasome from yeast At $2.4 \AA$ resolution. Nature 386, 463-471 (1997).

9. Arendt, C. S. \& Hochstrasser, M. Identification of the yeast 20 S proteasome catalytic centers and subunit interactions required for active-site formation. Proc. Natl. Acad. Sci. U. S. A. 94, 7156-7161 (1997).

10. Groll, M. et al. A gated channel into the proteasome core particle. Nat. Struct. Biol. 7, 10621067 (2000).

11. Baumeister, W. et al. The proteasome: paradigm of a self-compartmentalizing protease. Cell 92, 367-380 (1998).

12. Köhler, A. et al. The axial channel of the proteasome core particle is gated by the Rpt2 ATPase and controls both substrate entry and product release. Mol. Cell 7, 1143-1152 (2001).

13. Finley, D., Chen, X. \& Walters, K. J. Gates, channels, and switches: Elements of the proteasome machine. Trends Biochem. Sci. 41, 77-93 (2016).

14. Smith, D. M. et al. Docking of the proteasomal ATPases' carboxyl termini in the 20S proteasome's alpha ring opens the gate for substrate entry. Mol Cell 27, 731-744 (2007).

15. Rabl, J. et al. Mechanism of Gate Opening in the 20S Proteasome by the Proteasomal ATPases. Mol. Cell 30, 360-368 (2008).

16. Whitby, F. G. et al. Structural basis for the activation of $20 \mathrm{~S}$ proteasomes by $11 \mathrm{~S}$ regulators. Nature 408, 115-120 (2000).

17. Dong, Y. et al. Cryo-EM structures and dynamics of substrate-engaged human 26S proteasome. Nature (2018). doi:10.1038/s41586-018-0736-4

18. de la Peña, A. H., Goodall, E. A., Gates, S. N., Lander, G. C. \& Martin, A. Substrateengaged 26S proteasome structures reveal mechanisms for ATP-hydrolysis-driven translocation. Science. 362, eaav0725 (2018).

19. Eisele, M. R. et al. Expanded Coverage of the 26S Proteasome Conformational Landscape Reveals Mechanisms of Peptidase Gating. Cell Rep. 24, 1301-1315.e5 (2018).

20. Zhu, Y. et al. Structural mechanism for nucleotide-driven remodeling of the AAA-ATPase unfoldase in the activated human $26 \mathrm{~S}$ proteasome. Nat. Commun. 9, 1-12 (2018).

21. Chu, P. M. et al. Identification, purification, and characterization of a high molecular weight, ATP-dependent activator (PA700) of the 20 S proteasome. J Biol Chem 269, 3539-3547 
(1994).

22. Gillette, T. G., Kumar, B., Thompson, D., Slaughter, C. A. \& DeMartino, G. N. Differential roles of the $\mathrm{COOH}$ termini of AAA subunits of PA700 (19S regulator) in asymmetric assembly and activation of the 26 S proteasome. J. Biol. Chem. 283, 31813-31822 (2008).

23. Chondrogianni, N., Sakellari, M., Lefaki, M., Papaevgeniou, N. \& Gonos, E. S. Proteasome activation delays aging in vitro and in vivo. Free Radical Biology and Medicine 71, 303320 (2014).

24. Chondrogianni, N. et al. Overexpression of proteasome $\beta 5$ subunit increases the amount of assembled proteasome and confers ameliorated response to oxidative stress and higher survival rates. J. Biol. Chem. 280, 11840-50 (2005).

25. Lee, J. H. et al. Facilitated Tau Degradation by USP14 Aptamers via Enhanced Proteasome Activity. Sci. Rep. 5, 10757 (2015).

26. Sadre-Bazzaz, K., Whitby, F. G., Robinson, H., Formosa, T. \& Hill, C. P. Structure of a Blm10 complex reveals common mechanisms for proteasome binding and gate opening. Mol. Cell 37, 728-735 (2010).

27. Majumder, P. et al. Cryo-EM structures of the archaeal PAN-proteasome reveal an aroundthe-ring ATPase cycle. Proc. Natl. Acad. Sci. 116, 2-7 (2019).

28. Yu, Y. et al. Interactions of PAN's C-termini with archaeal 20 S proteasome and implications for the eukaryotic proteasome-ATPase interactions. EMBO J. 29, 692-702 (2010).

29. Rabl, J. et al. Mechanism of Gate Opening in the 20S Proteasome by the Proteasomal ATPases. Mol. Cell 30, 360-368 (2008).

30. Zwickl, P., Ng, D., Woo, K. M., Klenk, H. P. \& Goldberg, A. L. An archaebacterial ATPase, homologous to ATPases in the eukaryotic $26 \mathrm{~S}$ proteasome, activates protein breakdown by $20 \mathrm{~S}$ proteasomes. J. Biol. Chem. 274, 26008-26014 (1999).

31. Smith, D. M. et al. Docking of the proteasomal ATPases' carboxyl termini in the 20S proteasome's a-ring opens the gate for substrate entry. Mol. Cell 27, 731-744 (2007).

32. Rock, K. L. et al. Inhibitors of the proteasome block the degradation of most cell proteins and the generation of peptides presented on MHC class I molecules. Cell 78, 761-771 (1994).

33. Lee, B.-H. H. et al. Enhancement of proteasome activity by a small-molecule inhibitor of USP14. Nature 467, 179-184 (2010).

34. Boselli, M. et al. An inhibitor of the proteasomal deubiquitinating enzyme USP14 induces tau elimination in cultured neurons. J. Biol. Chem. 292, 19209-19225 (2017).

35. Ciechanover, A. \& Brundin, P. The ubiquitin proteasome system in neurodegenerative diseases: Sometimes the chicken, sometimes the egg. Neuron 40, 427-446 (2003).

36. Han, D. H. et al. Direct cellular delivery of human proteasomes to delay tau aggregation. Nat. Commun. 5, 5633 (2014).

37. Choi, W. H. et al. Open-gate mutants of the mammalian proteasome show enhanced ubiquitin-conjugate degradation. Nat. Commun. 7, 10963 (2016).

38. Forster, A., Whitby, F. G. \& Hill, C. P. The pore of activated 20 S proteasomes has an ordered 7-fold symmetric conformation. Embo J 22, 4356-4364 (2003).

39. Unno, M. et al. Structure determination of the constitutive $20 \mathrm{~S}$ proteasome from bovine liver at 2.75 A resolution. J. Biochem. 131, 171-173 (2002).

40. Kisselev, A. F., Kaganovich, D. \& Goldberg, A. L. Binding of hydrophobic peptides to several non-catalytic sites promotes peptide hydrolysis by all active sites of $20 \mathrm{~S}$ proteasomes. J. Biol. Chem. 277, 22260-22270 (2002).

41. Thibaudeau, T. A., Anderson, R. T. \& Smith, D. M. A common mechanism of proteasome impairment by neurodegenerative disease-associated oligomers. Nat. Commun. 9, 1097 (2018).

42. Kayed, R. et al. Common structure of soluble amyloid oligomers implies common mechanism of pathogenesis. Science 300, 486-489 (2003). 
43. Smith, D. M. et al. ATP binding to PAN or the 26S ATPases causes association with the $20 \mathrm{~S}$ proteasome, gate opening, and translocation of unfolded proteins. Mol. Cell 20, 687698 (2005). 


\section{General Discussion}

The structural evolution of regulated proteases was driven by the need to protect proteolytic activity from the cellular milieu, but still have the capacity to degrade select proteins in a regulated manner. The substrate-entry gate in the $20 \mathrm{~S}$ proteasome thus plays a critical role in proteasome function and in cellular proteostasis. Maintaining proteostasis is especially important for neurons due to their complex architecture, long lifespan, and inability to dilute aggregate load by cell division $^{1}$. Moreover, proteasomal degradation is critical for normal functioning of neuronal synapses, including synaptic protein turnover, plasticity, and long-term memory formation, which rely on tightly controlled changes in the proteome ${ }^{1-4}$. As dysfunction in proteasomal degradation has been implicated in many human diseases, biochemical and structural analysis of the proteasome is essential to advance our understanding of its action and regulation mechanisms.

\section{Mechanism of proteasome impairment by protein oligomers}

It is well established that proteasome function is decreased in neurodegenerative diseases, and impaired function has been implicated in the development of many neurodegenerative diseases, including Alzheimer's, Parkinson's, and Huntington's disease ${ }^{5-9}$. Study 1 identified a common mechanism of proteasome impairment by neurodegenerative disease associated protein oligomers. We showed that oligomers from three different ND associated proteins adopt a similar 3D conformation and stabilize the proteasome gate in the closed conformation, thereby restricting substrate entry into the catalytic 205 proteasome core. Taken together, biochemical data from Study 1 suggests oligomers stabilize the closed gate by binding to the $\alpha$-intersubunit cleft on the side of the proteasome (Figure 1).

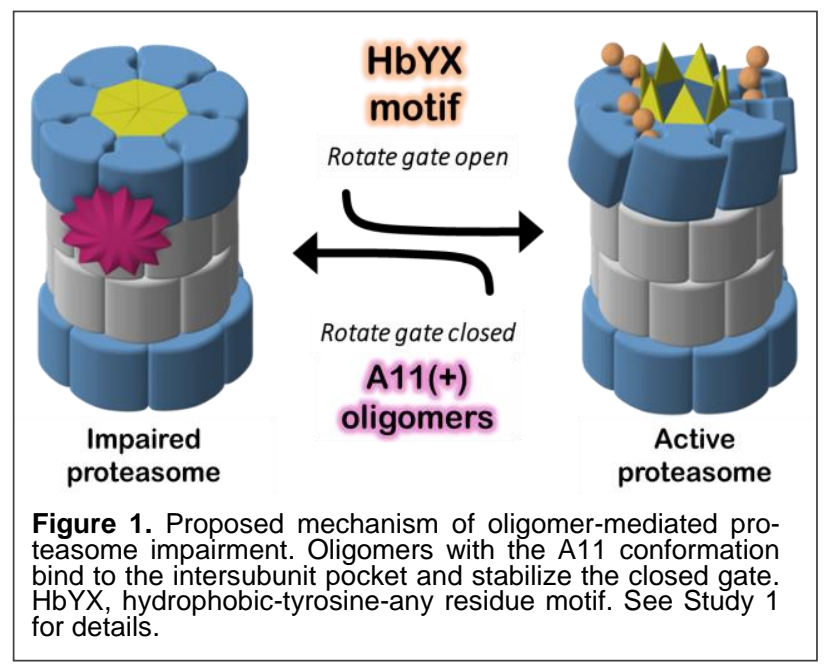




\section{I.A. Targeting oligomer-proteasome interactions}

Protein-protein interactions (PPIs) represent a vast class of therapeutic targets both inside and outside the cell. Twenty years ago, PPIs were deemed intractable drug targets ${ }^{10}$. Thanks to recent advances in our understanding of PPIs, more than 40 PPIs have been targeted and several PPIinhibitors have reached clinical trials ${ }^{11-13}$. Increased proteasome activity has been shown to have a protective cellular effect and extend lifespan ${ }^{14-17}$, therefore preventing oligomer-proteasome interactions has the potential to restore proteasome function in neurodegenerative disease. Understanding the structural interactions of oligomers with the proteasome is the foundation for designing protein-protein interaction (PPI) inhibitors that prevent oligomers from binding to the proteasome.

Mass spectrometry combined with hydrogen/deuterium $(H / D)$ exchange is used to study the conformation and dynamics of proteins. H/D exchange can be used in a comparative manner to identify changes in protein conformation and dynamics induced by ligand binding or protein-protein interactions ${ }^{18,19}$. Analysis of oligomer-proteasome interactions with $\mathrm{H} / \mathrm{D}$ exchange techniques can provide identification of the oligomer binding site, and further elucidate changes in proteasome gate dynamics due to oligomer-proteasome interactions.

Chemical crosslinking is another important tool for mapping protein three-dimensional structures and protein-protein interactions ${ }^{20}$. In recent years, new crosslinking/mass spectrometry (XL-MS) approaches have been developed to study proteasome structural conformations and proteasomeinteracting proteins ${ }^{21-23}$. For example, using two cross-linkers, bis (sulfosuccinimidyl) suberate and its water-insoluble analog disuccinimidyl suberate (DSS), Song et al. (2016) mapped the noncovalent interactions among 20 S proteasome subunits in breast cancer cells ${ }^{21}$. Using a disuccinimidyl sulfoxide-based (DSSO) cross-linking and mass spectrometry platform, Wang et al. (2017) mapped the interactions of Ecm29 within itself and with proteasome subunits and determined the architecture of the Ecm29-proteasome complex with integrative structure modeling ${ }^{23}$. 
Thus, HD exchange and XL-MS chemical crosslinking approaches can potentially identify and structurally characterize the oligomer binding site on the proteasome. The potential druggability of the oligomer-proteasome interaction can be assessed by analysis of the geometrical parameters and residue properties of the binding site ${ }^{11}$.

\section{Proteasome gate activation by synthetic molecules}

Study 2 characterized the mechanism of $\mathrm{HbYX}$ induced proteasome gate opening and identified $\mathrm{HbYX}$-based di-peptides as proteasome gate-activators. Notably, we developed a novel HbYX dipeptide, ZYA (Figure 2). ZYA promotes proteasome gate opening and rescues proteasome activity from oligomer-mediated impairment, supporting the $\mathrm{HbYX}$ mechanism as a potential drug target to restore proteasome activity in neurodegenerative diseases. These data provide a mechanistic framework to develop small molecules directly activate the proteasome gate, thereby increasing proteasome activity and protein degradation.

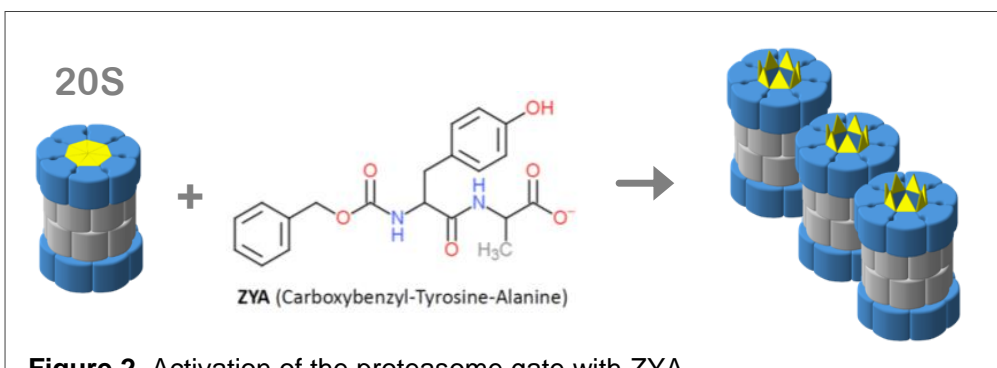

Figure 2. Activation of the proteasome gate with ZYA.

\section{II.A. Mechanism of HbYX-motif proteasome gate opening}

Detailed knowledge of the HbYX gate mechanism is essential for the rational design of gateactivating molecules. The first proteasome gating studies identified the mechanism of proteasomal ATPase-induced gate opening in the $20 \mathrm{~S}$ by studying the archaeal 20S-PAN ATPase (archaeal homolog of 19S ATPases) complex. The Goldberg Lab and colleagues demonstrated that docking of the PAN ATPases' C-termini (containing the conserved HbYX motif) in to the 20S proteasome $\alpha$-ring opens the $20 \mathrm{~S}$ gate by a "key-in-a-lock" mechanism ${ }^{24-26}$. Furthermore, sevenresidue or longer peptides from PAN's C-terminus containing the HbYX motif can by themselves cause a rotation of the $\alpha$-subunits to induce gate opening ${ }^{26}$. The process of $19 S-20 S$ complex formation and gate opening is much more complex than PAN-20S interactions, due to the 
presence of six different 19S ATPases with distinct C-termini and seven distinct a-intersubunit pockets in the eukaryotic 20 s proteasomes. Nonetheless, the HbYX motif "key-in-a-lock" mechanism appeared to be conserved from archaea to mammalian proteasomes as gate opening could be induced by seven-residue peptides from the 19S ATPase subunits C-termini (Rpt2 and Rpt5, containing the HbYX motif) 25 .

Recent single-particle cryo-electron microscopy (cryo-EM) studies of yeast and human 26S proteasomes have revealed a complex mechanism for the 19S ATPase hydrolysis cycle, gate opening, substrate unfolding and translocation into the $20 \mathrm{~S}$ core $28-34$. These studies have presented structures of multiple 26S conformational states during the ATP hydrolysis cycle and substrate processing, the majority of which have a closed $20 \mathrm{~S}$ gate. Several structures show 19S ATPase C-termini containing the HbYX motif (Rpt 5 and 3, plus Rpt 2 in some conformational states) docked into $\alpha$-intersubunit pockets in the absence of gate-opening. These data have been interested as evidence that the HbYX motif alone does not induce gate opening in eukaryotic proteasomes.

It is worth noting that cryo-EM analysis pf the $26 \mathrm{~S}$ proteasome is not without experimental uncertainty. Single-particle EM depends on the computational averaging of thousands of images of identical particles ${ }^{27}$. Since the $26 \mathrm{~S}$ proteasome adopts many different conformations during the ATP hydrolysis cycle, more homogeneous subsets of 26 conformations are generated using 2D and 3D classification procedures prior to computational averaging of the particle subset. Nonetheless, the resultant 26S molecular structure is still derived from the averaged density of 10,000 to $>200,00026$ particles $^{28}$. Averaging a subset of proteasome particles can obscure open-gated proteasomes if they are a minority within the subset. Although one or more 19S ATPase C-termini containing the HbYX motif are docked into $\alpha$-intersubunit pockets in several closed-gate $26 \mathrm{~S}$ structures, the local resolution is $>3.5 \AA^{28-34}$ which doesn't allow identification of specific side chain interactions between the HbYX motif and the a-subunits. Therefore, the precise position of the 
$\mathrm{HbYX}$ motif in the $\alpha$-intersubunit pocket and whether or not it is interacting with the conserved residues required for gate opening (e.g. K66, P16) cannot be determined at these resolutions.

Conflicting evidence between the biochemical and structural studies described above casts doubt on the utility of using $\mathrm{HbYX}$ derived molecules to activate mammalian proteasomes. Study 2 fills this knowledge gap by defining the mechanism of $\mathrm{HbYX}$ motif gate activation at the molecular level (i.e. displacement of P16 backbone outward from the 20 S pore axis resulting from a hydrogen bond with the HbYX tyrosine hydroxyl group) and designed a synthetic dipeptide (ZYA) capable of activating the mammalian 20S gate and increasing protein degradation. ZYA is an effective gate activator (increasing activity over 50 -fold) but has relatively low affinity for the proteasome ( 1 $\left.\mathrm{mM} \mathrm{K}_{\mathrm{d}}\right)$. Future structure-activity relationship (SAR) models of ZYA-derivatives combined with an iterative process of design and screening can potentially generate new gateactivating compounds with improved potency and drug-like characteristics.

\section{Future Perspectives}

Impaired proteasome function has been implicated in the development of many neurodegenerative diseases ${ }^{5-9}$, and increasing proteasome activity has been recognized as a promising new approach to delay the onset or ameliorate the symptoms of neurodegenerative and other protein misfolding disorders ${ }^{35-37}$. Taken together, Study 1 and Study 2 identify a mechanism of proteasome impairment by disease-associated oligomers and demonstrate that proteasome gateactivating molecules can increase protein degradation and overcome neurodegenerative-oligomer impairment. However, our studies were performed in vitro with purified proteasomes, and several important questions remain unanswered.

\section{III.A. In vivo proteasome activation}

While cultured cells, drosophila, and nematodes respond favorably to increased proteasome activity (e.g. via ectopic proteasome delivery, proteasome subunit overexpression, genetic open- 
gate mutations) ${ }^{14,17,38-40}$, these model systems are short-lived compared to humans and cannot recapitulate all aspects of human neurogenerative disease. Notably, pathological changes at molecular and cellular levels in human patients precedes the clinical onset by several years. In fact, neuron loss is apparent at the onset of neurological symptoms and that neuronal dysfunction and abnormal protein deposited in the brain are already detectable in asymptomatic carriers of a gene mutation of familial neurodegenerative disease. We do not know if pharmacologically activating the proteasome in an organism with preexisting disease pathology can restore cellular proteostasis and if this is enough to have a positive impact on neural function and human health.

\section{III.B. Crosstalk between the proteasome and autophagy}

Autophagy is responsible for nonspecific, bulk degradation of cytoplasmic components. Recent work has also revealed specific, autophagic degradation of polyubiquitinated proteins and aggregates $^{41,42}$, and dynamic activity regulation between the UPS and autophagy ${ }^{43-45}$. Even though several biochemical mechanisms underlying crosstalk between them have been suggested ${ }^{45,46}$, less is known about the effect of enhanced proteasome activity on autophagic flux. Studies have shown that increased proteasome activity (achieved with hyperactive $\alpha 3 \Delta \mathrm{N}$ proteasomes or by pharmacological inhibition of USP14) inhibit autophagy flux at the autophagosome-lysosome fusion step ${ }^{14,47}$. The mechanism of autophagy inhibition appears to involve enhanced proteasomal degradation of the autophagosome-lysosome fusion protein, UV radiation resistance-associated gene (UVRAG) $)^{14,47}, \mathrm{p} 53$, and $\mathrm{p} 62^{14}$. Consequently, although proteasome activation improved the clearance of aggregation-prone proteins and reduced the amount of oligomers ${ }^{14,47}$, these conditions also increased the formation of inclusion bodies from nonproteasomal substrates (such as huntingtin with expanded polyglutamine repeats) ${ }^{47}$ and the number of GFP-LC3 puncta (a marker of autophagosomal structures $)^{14}$. In contrast, other studies have shown that proteasome activation through genetic and pharmacological inhibition of USP14 corrected an in vivo model of 
impaired mitophagy ${ }^{48}$ and increased autophagic flux ${ }^{49,50}$, likely through suppression of K63 ubiquitination of Beclin $1^{50}$.

Taken together, these observations highlight the fact that while a strategy enhancing proteasome activity may be beneficial in the treatment of neurodegenerative disease, it may have caveats originating from crosstalk mechanisms between the UPS and autophagy. Thus, future efforts are needed to address the effect of proteasome activation on autophagy regulatory mechanisms and identify the molecular link between proteasome activity and autophagic flux. 


\section{References}

1. Tai, H.-C. \& Schuman, E. M. Ubiquitin, the proteasome and protein degradation in neuronal function and dysfunction. Nat. Rev. Neurosci. 9, 826-38 (2008).

2. Fonseca, R., Vabulas, R. M., Hartl, F. U., Bonhoeffer, T. \& Nägerl, U. V. A balance of protein synthesis and proteasome-dependent degradation determines the maintenance of LTP. Neuron 52, 239-245 (2006).

3. Djakovic, S. N., Schwarz, L. A., Barylko, B., DeMartino, G. N. \& Patrick, G. N. Regulation of the proteasome by neuronal activity and calcium/calmodulin-dependent protein kinase II. J. Biol. Chem. 284, 26655-26665 (2009).

4. Asano, S. et al. Proteasomes. A molecular census of $26 \mathrm{~S}$ proteasomes in intact neurons. Science (80-. ). 347, 439-442 (2015).

5. Rubinsztein, D. C. The roles of intracellular protein-degradation pathways in neurodegeneration. Nature 443, 780-786 (2006).

6. Ciechanover, A. \& Brundin, P. The ubiquitin proteasome system in neurodegenerative diseases: Sometimes the chicken, sometimes the egg. Neuron 40, 427-446 (2003).

7. McNaught, K. S., Olanow, C. W., Halliwell, B., Isacson, O. \& Jenner, P. Failure of the ubiquitin-proteasome system in Parkinson's disease. Nat. Rev. Neurosci. 2, 589-594 (2001).

8. McKinnon, C. \& Tabrizi, S. J. The ubiquitin-proteasome system in neurodegeneration. Antioxid. Redox Signal. 5, 2302-2321 (2014).

9. Ortega, Z., Díaz-Hernández, M. \& Lucas, J. J. Is the ubiquitin-proteasome system impaired in Huntington's disease? Cell. Mol. Life Sci. 64, 2245-2257 (2007).

10. Arkin, M. R., Tang, Y. \& Wells, J. A. Small-molecule inhibitors of protein-protein interactions: progressing toward the reality. Chem. Biol. 21, 1102-14 (2014).

11. Basse, M. J. et al. 2P2ldb: a structural database dedicated to orthosteric modulation of protein-protein interactions. Nucleic Acids Res. 41, D824-D827 (2012).

12. Higueruelo, A. P. et al. Atomic Interactions and Profile of Small Molecules Disrupting Protein-Protein Interfaces: the TIMBAL Database. Chem. Biol. Drug Des. 74, 457-467 (2009).

13. Labbé, C. M., Laconde, G., Kuenemann, M. A., Villoutreix, B. O. \& Sperandio, O. iPPI-DB: a manually curated and interactive database of small non-peptide inhibitors of proteinprotein interactions. Drug Discov. Today 18, 958-968 (2013).

14. Choi, W. H. et al. Open-gate mutants of the mammalian proteasome show enhanced ubiquitin-conjugate degradation. Nat. Commun. 7, 10963 (2016).

15. Lee, B. H. et al. Enhancement of proteasome activity by a small-molecule inhibitor of USP14. Nature 467, 179-184 (2010).

16. Lee, J. H. et al. Facilitated Tau Degradation by USP14 Aptamers via Enhanced Proteasome Activity. Sci. Rep. 5, 10757 (2015).

17. Nguyen, N. N. et al. Proteasome $\beta 5$ subunit overexpression improves proteostasis during aging and extends lifespan in Drosophila melanogaster. Sci. Rep. 9, 3170 (2019).

18. Ramirez-Sarmiento, C. A. \& Komives, E. A. Hydrogen-deuterium exchange mass spectrometry reveals folding and allostery in protein-protein interactions. Methods 144, 4352 (2018).

19. Percy, A. J., Rey, M., Burns, K. M. \& Schriemer, D. C. Probing protein interactions with hydrogen/deuterium exchange and mass spectrometry-A review. Anal. Chim. Acta 721, 7-21 (2012).

20. Yu, C. \& Huang, L. Cross-Linking Mass Spectrometry: An Emerging Technology for Interactomics and Structural Biology. Anal. Chem. 90, 144-165 (2018).

21. Song, $\mathrm{H}$. et al. Gel-based chemical cross-linking analysis of $20 \mathrm{~S}$ proteasome subunit- 
subunit interactions in breast cancer. J Huazhong Univ Sci Technol. Med Sc 36, 564-570 (2016).

22. Wang, $X$. et al. Molecular details underlying dynamic structures and regulation of the human $26 S$ proteasome. Mol. Cell. Proteomics 16, 840-854 (2017).

23. Wang, $X$. et al. The proteasome-interacting Ecm29 protein disassembles the 26S proteasome in response to oxidative stress. J. Biol. Chem. 292, 16310-16320 (2017).

24. $\mathrm{Yu}, \mathrm{Y}$. et al. Interactions of PAN's $\mathrm{C}$-termini with archaeal $20 \mathrm{~S}$ proteasome and implications for the eukaryotic proteasome-ATPase interactions. EMBO J. 29, 692-702 (2010).

25. Smith, D. M. et al. Docking of the proteasomal ATPases' carboxyl termini in the 20S proteasome's alpha ring opens the gate for substrate entry. Mol Cell 27, 731-744 (2007).

26. Rabl, J. et al. Mechanism of Gate Opening in the 20S Proteasome by the Proteasomal ATPases. Mol. Cell 30, 360-368 (2008).

27. Cheng, Y., Grigorieff, N., Penczek, P. A. \& Walz, T. A primer to single-particle cryo-electron microscopy. Cell 161, 438-449 (2015).

28. Dong, $Y$. et al. Cryo-EM structures and dynamics of substrate-engaged human $26 \mathrm{~S}$ proteasome. Nature 565, 49-55 (2019)

29. Wehmer, M. et al. Structural insights into the functional cycle of the ATPase module of the 26 S proteasome. Proc. Natl. Acad. Sci. 114, 201621129 (2017).

30. de la Peña, A. H., Goodall, E. A., Gates, S. N., Lander, G. C. \& Martin, A. Substrateengaged 26S proteasome structures reveal mechanisms for ATP-hydrolysis-driven translocation. Science 362, eaav0725 (2018).

31. Eisele, M. R. et al. Expanded Coverage of the 26S Proteasome Conformational Landscape Reveals Mechanisms of Peptidase Gating. Cell Rep. 24, 1301-1315 (2018).

32. Chen, S. et al. Structural basis for dynamic regulation of the human $26 \mathrm{~S}$ proteasome. Proc. Natl. Acad. Sci. 113, 12991-12996 (2016).

33. Śledź, P. et al. Structure of the $26 \mathrm{~S}$ proteasome with ATP-yS bound provides insights into the mechanism of nucleotide-dependent substrate translocation. Proc. Natl. Acad. Sci. 110, 7264-7269 (2013).

34. Unverdorben, P. et al. Deep classification of a large cryo-EM dataset defines the conformational landscape of the $26 \mathrm{~S}$ proteasome. Proc. Natl. Acad. Sci. 111, 5544-5549 (2014).

35. Opoku-Nsiah, K. A. \& Gestwicki, J. E. Aim for the core: suitability of the ubiquitinindependent $20 \mathrm{~S}$ proteasome as a drug target in neurodegeneration. Transl. Res. 198, 4857 (2018).

36. Myeku, N. \& Duff, K. E. Targeting the 26S Proteasome To Protect Against Proteotoxic Diseases. Trends Mol. Med. 24, 18-29 (2018).

37. Njomen, E. \& Tepe, J. J. Proteasome Activation as a New Therapeutic Approach To Target Proteotoxic Disorders. J. Med. Chem. 9b00101 (2019). doi:10.1021/acs.jmedchem.9b00101

38. Han, D. H. et al. Direct cellular delivery of human proteasomes to delay tau aggregation. Nat. Commun. 5, 5633 (2014).

39. Hamer, G., Matilainen, O. \& Holmberg, C. I. A photoconvertible reporter of the ubiquitinproteasome system in vivo. Nat. Methods 7, 473-478 (2010).

40. Chondrogianni, N., Georgila, K., Kourtis, N., Tavernarakis, N. \& Gonos, E. S. 20 S proteasome activation promotes life span extension and resistance to proteotoxicity in Caenorhabditis elegans. FASEB J. 29, 611-22 (2015).

41. Lee, M. J., Lee, J. H. \& Rubinsztein, D. C. Tau degradation: The ubiquitin-proteasome system versus the autophagy-lysosome system. Progress in Neurobiology 105, 49-59 (2013).

42. Kim, P. K., Hailey, D. W., Mullen, R. T. \& Lippincott-Schwartz, J. Ubiquitin signals autophagic degradation of cytosolic proteins and peroxisomes. Proc. Natl. Acad. Sci. 105, 
20567-74 (2008).

43. Zhao, J. \& Goldberg, A. L. Coordinate regulation of autophagy and the ubiquitin proteasome system by MTOR. Autophagy 12, 1967-1970 (2016).

44. Kageyama, S. et al. Proteasome Dysfunction Activates Autophagy and the Keap1-Nrf2 Pathway. J. Biol. Chem. 289, 24944-24955 (2014).

45. Nam, T., Han, J. H., Devkota, S. \& Lee, H.-W. Emerging Paradigm of Crosstalk between Autophagy and the Ubiquitin-Proteasome System. Mol. Cells 40, 897-905 (2017).

46. Ji, C. H. \& Kwon, Y. T. Crosstalk and Interplay between the Ubiquitin-Proteasome System and Autophagy. Mol. Cells 40, 441-449 (2017).

47. Kim, E. et al. Dual Function of USP14 Deubiquitinase in Cellular Proteasomal Activity and Autophagic Flux. Cell Rep. 24, 732-743 (2018).

48. Chakraborty, J. et al. USP14 inhibition corrects an in vivo model of impaired mitophagy. EMBO Mol. Med. 10, e9014 (2018).

49. Boselli, M. et al. An inhibitor of the proteasomal deubiquitinating enzyme USP14 induces tau elimination in cultured neurons. J. Biol. Chem. 292, 19209-19225 (2017).

50. $\mathrm{Xu}, \mathrm{D}$. et al. USP14 regulates autophagy by suppressing K63 ubiquitination of Beclin 1. Genes Dev. 30, 1718-1730 (2016). 


\section{Curriculum Vitae \\ Tiffany A. Thibaudeau}

Department of Molecular Biosciences

(Work): (847) 491-3714

Northwestern University

(Cell): (502) 777-1821

Richard I. Morimoto Laboratory

2205 Tech Drive, Hogan 1-500

Evanston, IL 60208

Email: eco.ri@icloud.com; tiffany.thibaudeau@northwestern.edu

Residence: 3639 N. Racine Ave \#1, Chicago, IL 60640

Citizenship: United States of America

Current Position: Postdoctoral Fellow, Northwestern University

\section{EDUCATION}

Ph.D.

West Virginia University

2019

Degree in Biochemistry and Molecular Biology, School of Medicine, Morgantown, WV. Laboratory of David M. Smith Ph.D.

B. A.

University of Louisville

2011

Degree in Psychology, Department of Psychological and Brain Sciences, Louisville, KY.

\section{RESEARCH EXPERIENCE}

Postdoctoral Fellow

Northwestern University

2019-pres

Department of Molecular Biosciences, Evanston, IL.

Researching mechanisms underlying coordinated regulation coordination of the ubiquitin proteasome and autophagy-lysosome systems within and across tissues under Dr. Richard I. Morimoto.

\section{Graduate Research Assistant West Virginia University}

2014-2019

School of Medicine, Department of Biochemistry and Molecular Biology, Morgantown, WV.

Research project focused on regulating proteasome substrate entry in neurodegenerative disease under Dr. David M. Smith.

Research Technician

West Virginia University

2011-2014

School of Medicine, Department of Biochemistry and Molecular Biology, Morgantown, WV.

Performed biochemical, biophysical, structural, pharmacological, computational, molecular, and cell biological assays to study proteasome structure and function under the guidance of Dr. David M. Smith.

Honors Thesis Research

Department of Psychological and Brain Sciences, Louisville, KY.

Researched the role of source misattribution to false-positive results in the autobiographical implicit association test under Dr. Keith Lyle. 


\section{SCIENTIFIC PRESENTATIONS}

Invited Oral Presentations

American Society for Biology and Molecular Biology (ASBMB) Annual Convention, Spotlight Session: Protein and Enzyme Allostery. April 25, 2017. Chicago, IL. "A common mechanism of proteasomal impairment by neurodegenerative disease associated oligomers"

Pittsburgh Ubiquitin-Proteasome Meeting. May 12, 2016. University of Pittsburgh, "Proteasome impairment by structurally similar oligomers"

Poster Presentations

American Society for Biology and Molecular Biology (ASBMB) Annual Convention, April 2017. Chicago, IL. "A common mechanism of proteasomal impairment by neurodegenerative disease associated oligomers"

E.J. Van Liere Memorial Convocation \& HSC Research Day, April 2016. Morgantown, WV. "Structurally similar neurotoxic oligomers impair the proteasome"

Association for Psychological Science (APS) $23^{\text {rd }}$ Annual Convention. May, 2011. Washington, D.C. "How actions become associated with truth: Effects of performance, imagination, and reading on the autobiographical implicit association test"

\section{AWARDS AND HONORS}

Travel award, WVU Graduate Office, ASBMB / Experimental Biology (EB) Annual Convention held in Chicago, IL. April 22-26, 2017.

$1^{\text {st }}$ Place Poster Presentation, 2016 E.J. Van Liere Memorial Convocation \& HSC Research Day, Morgantown, WV

Travel award, $23^{\text {rd }}$ American Psychological Society Annual Convention held in Washington, D.C., May 24-26, 2011.

\section{MENTORING EXPERIENCE}

Trainees

Matthew Dombrowski - Undergraduate student

2017-2019

Pooja Warrier - Honors undergraduate student

2016-2017

Taylor Thomas - Biochemistry Ph.D. student

2016

Raymond Anderson - Biochemistry Ph.D. student

2015

Jordanna Bowser - Honors undergraduate student

2013

Tutoring

WVU School of Pharmacy students - BIOC 531

2015-2017

WVU School of Dentistry students - BIOC 531

2015-2017

UofL Psychology 101 students

2010-2011 


\section{PROFESSIONAL SOCIETY MEMBERSHIPS}

AAPS - American Association of Pharmaceutical Scientists 2017-Pres.

ASBMB - American Society for Biochemistry and Molecular Biology 2014-Pres.

SfN - Society for Neuroscience 2009-Pres.

APS - Association for Psychological Science 2009-2011

Golden Key International Honor Society 2008-2011

Psi-Chi International Psychology Honor Society 2008-2011

\section{REGISTERED STUDENT ORGANIZATION MEMBERSHIPS}

$\begin{array}{lll}\text { AAPS WVU Student Chapter } & \text { Member } & \text { 2017-2019 } \\ \text { AAPS 10th Annual Symposium } & \text { Judge } & 2017 \\ \text { WVU Cell Biology Training Program } & \text { Member } & 2012-\text { Pres. } \\ \text { WVU Cell Biology Training Program } & \text { Secretary } & 2014-2016 \\ 1^{\text {st }} \text { Annual Appalachian Regional Cell Conference } & \text { Planning Committee } & 2012\end{array}$

\section{RESEARCH INTERESTS}

Protein structure/function, protein misfolding, protein degradation, proteostasis network, ubiquitin proteasome system, neurodegeneration, rational drug design, activation of the proteasome, proteasome inhibition, neurotoxic oligomers.

\section{BIBLIOGRAPHY}

\section{Published}

Thibaudeau, T. A., Anderson, R. T., \& Smith, D. M. (2018). A common mechanism of proteasome impairment by neurodegenerative disease-associated oligomers. Nature communications 9(1), 1097.

Thibaudeau, T. A. \& Smith, D. M. (2019) A practical review of proteasome pharmacology. Pharmacological Reviews 71(2), 170-197.

\section{In Preparation}

Thibaudeau, T. A. \& Smith, D. M. (2019) Activation of proteasome gate-opening by mutagenesis and $\mathrm{HbYX}$ peptide mimetics. 